Remediation Guidance for Major Airports after a Bioterrorist Attack

T. Carlsen, E. Raber, D. Macqueen, L. Berg, J. Coty, G. Brown, C. Fish, K. Folks, S. Mancieri, D. Canter, J. Kempter

June 22, 2012 
This document was prepared as an account of work sponsored by an agency of the United States government. Neither the United States government nor Lawrence Livermore National Security, LLC, nor any of their employees makes any warranty, expressed or implied, or assumes any legal liability or responsibility for the accuracy, completeness, or usefulness of any information, apparatus, product, or process disclosed, or represents that its use would not infringe privately owned rights. Reference herein to any specific commercial product, process, or service by trade name, trademark, manufacturer, or otherwise does not necessarily constitute or imply its endorsement, recommendation, or favoring by the United States government or Lawrence Livermore National Security, LLC. The views and opinions of authors expressed herein do not necessarily state or reflect those of the United States government or Lawrence Livermore National Security, LLC, and shall not be used for advertising or product endorsement purposes.

This work performed under the auspices of the U.S. Department of Energy by Lawrence Livermore National Laboratory under Contract DE-AC52-07NA27344. 
it

LLNL-TR-562207

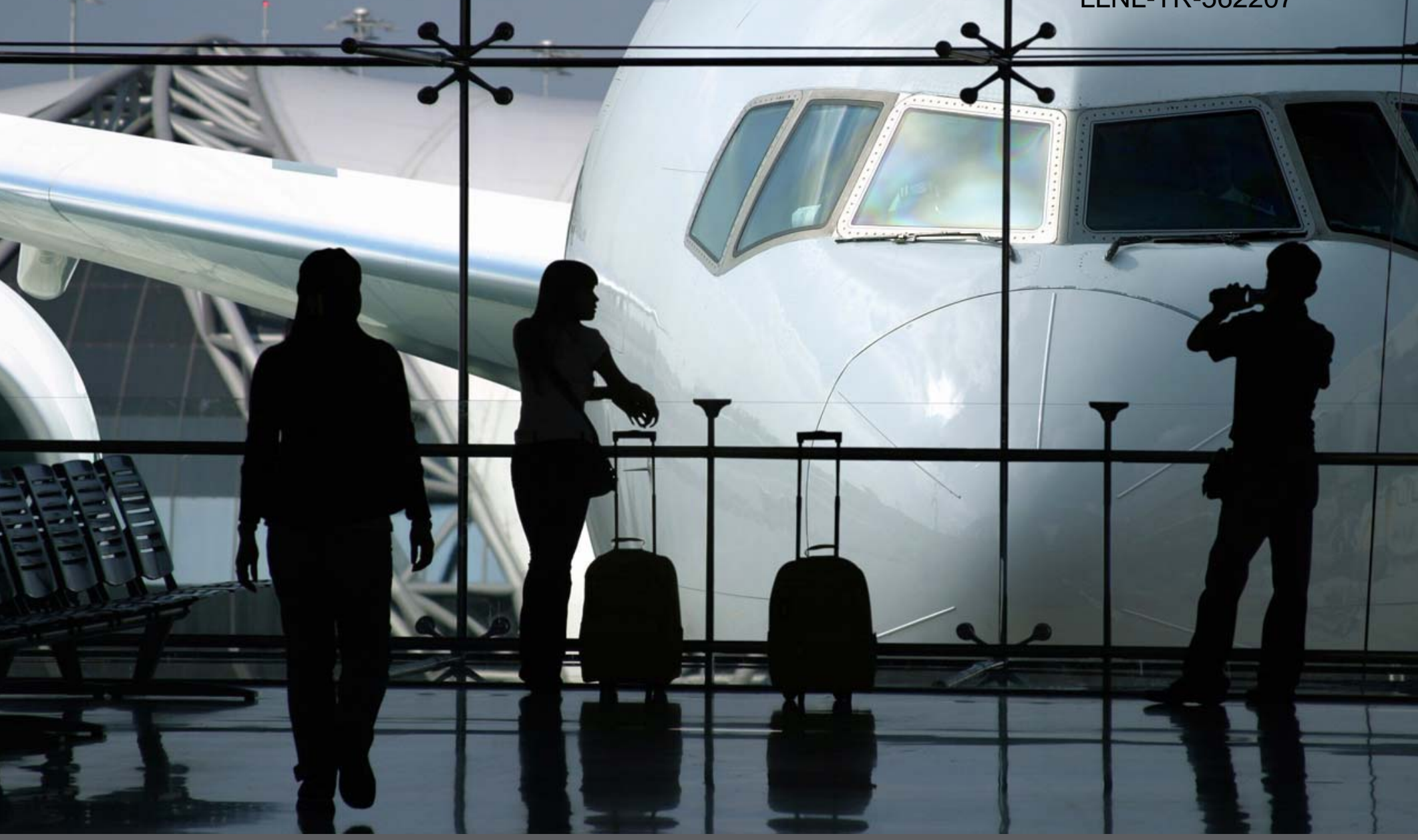

Remediation Guidance for Major Airports after a Bioterrorist Attack

November 2008

Re-issued June 2012
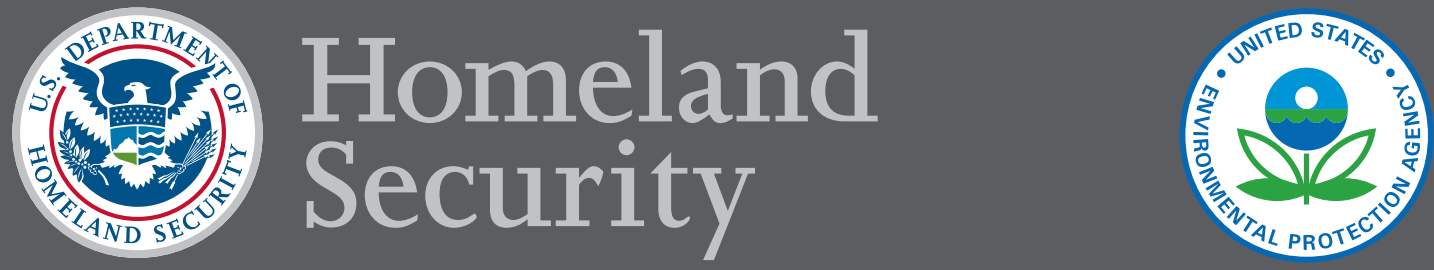



\section{Remediation Guidance for Major Airports after a Bioterrorist Attack}

\section{November 2008}

Re-issued June 2012

Donald Bansleben

Chemical/Biological Division

Science and Technology Directorate

U.S. Department of Homeland Security

Donald.Bansleben@dhs.gov
Erica Canzler

Office of Emergency Management

U.S. Environmental Protection Agency Canzler.Erica@epamail.epa.gov 



\section{Participants}

The following individuals contributed their time and expertise to writing this document:

Tina Carlsen

Ellen Raber

Don MacQueen

Lindee Berg

Robert Kirvel

Jessie Coty

Wendy Wilson

Gary Brown

Craig Fish

Richard Michalik

Karen Folks

Saverio Mancieri

Dorothy Canter

Jeff Kempter
Lawrence Livermore National Laboratory

Lawrence Livermore National Laboratory

Lawrence Livermore National Laboratory

Lawrence Livermore National Laboratory

Lawrence Livermore National Laboratory

Lawrence Livermore National Laboratory

Lawrence Livermore National Laboratory

Sandia National Laboratories

Lawrence Livermore National Laboratory

Lawrence Livermore National Laboratory

Lawrence Livermore National Laboratory

Lawrence Livermore National Laboratory

U.S. Environmental Protection Agency

U.S. Environmental Protection Agency

Appreciation is expressed to the following individuals for their contributions to reviewing this guidance:

Karen Burgan

Paul Lemieux

Blair Martin

Mark Durno

Tony Intrepido

Greg Knudson

Mike Shoemaker

Steve Calanog

Ken Martinez
U.S. Environmental Protection Agency

U.S. Environmental Protection Agency

U.S. Environmental Protection Agency

U.S. Environmental Protection Agency

U.S. Army, CHPPM

Armed Forces Radiobiology Research Institute

Armed Forces Radiobiology Research Institute

U.S. Environmental Protection Agency

Centers for Disease Control and Prevention 



\section{TABLEOF CONTENTS}

List of Acronyms ..v

Glossary .vii

Overview . .1

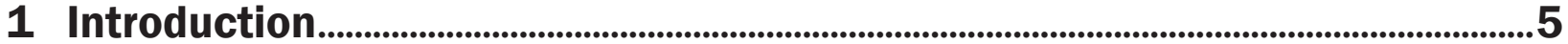

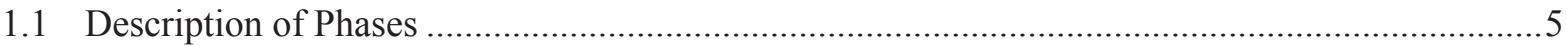

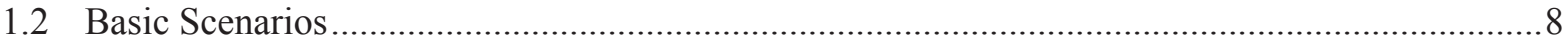

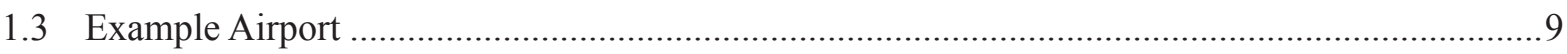

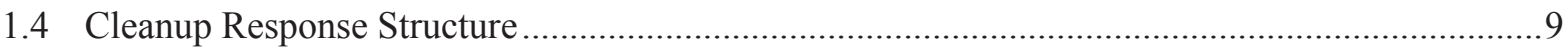

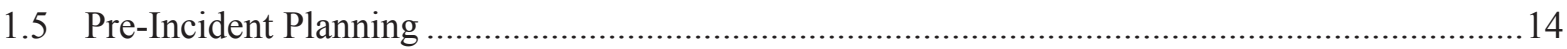

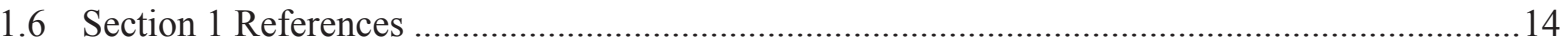

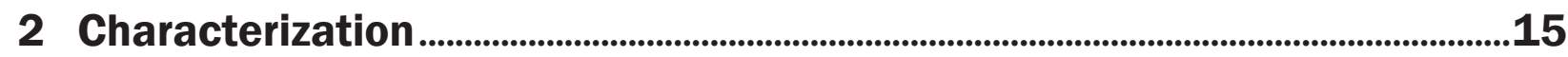

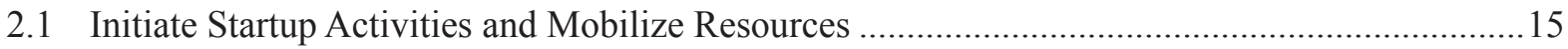

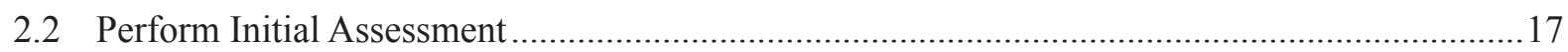

2.3 Plan for Characterization Environmental Sampling ..........................................................28

2.4 Prepare Incident-Specific, Operational, Characterization Sampling and Analysis Plan ..............32

2.5 Conduct Characterization Environmental Sampling; Evaluate Results .....................................32

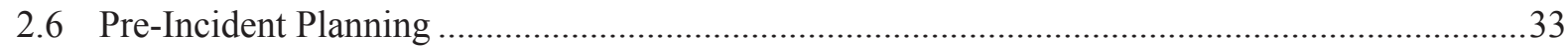

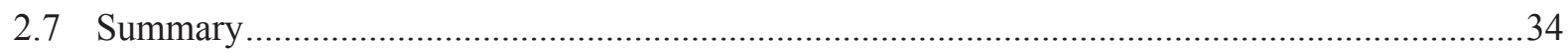

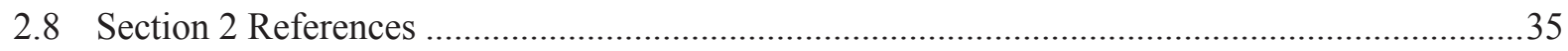

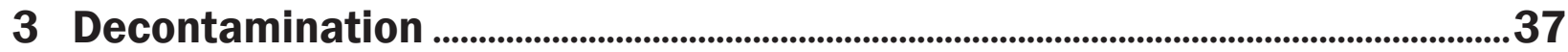

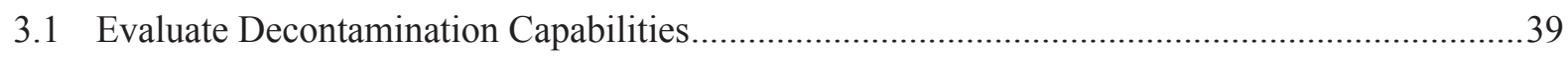

3.2 Establish Overall Goal for Successful Decontamination..........................................................39

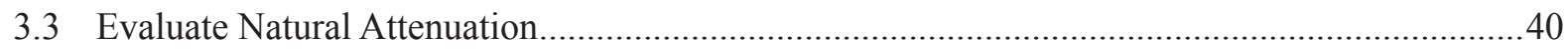

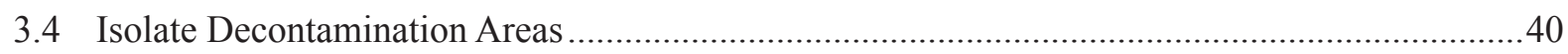

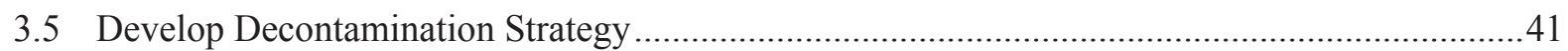

3.6 Prepare Remediation Action Plan and Related Documents..................................................48

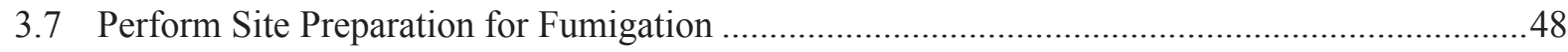

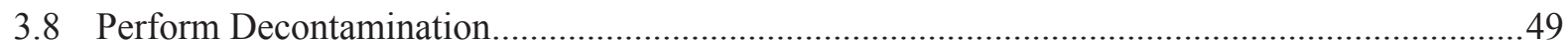

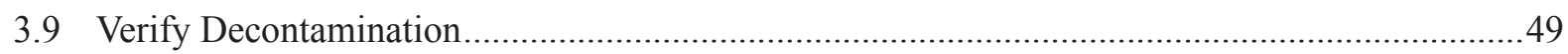

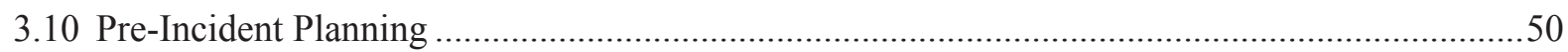

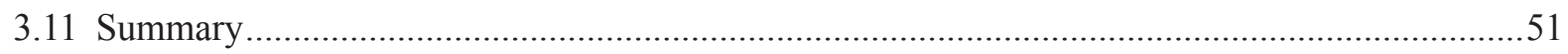

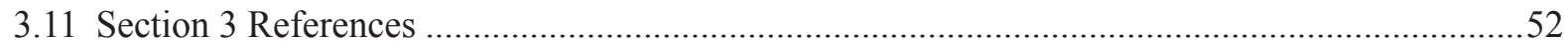

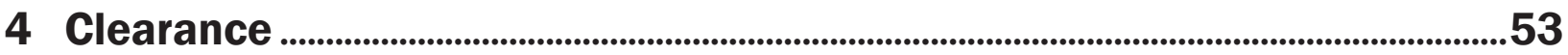

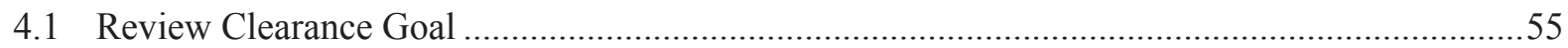

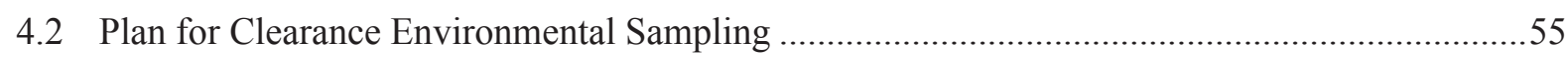

4.3 Prepare and Execute Clearance Environmental Sampling Plan .................................................59

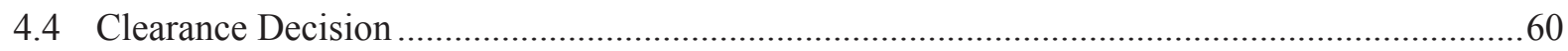

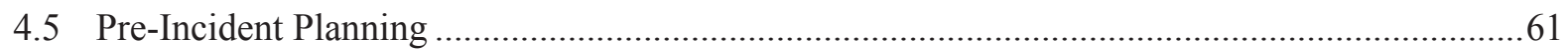

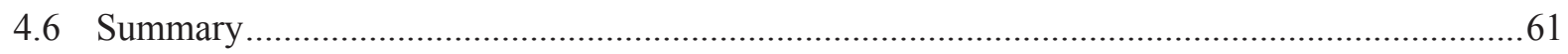

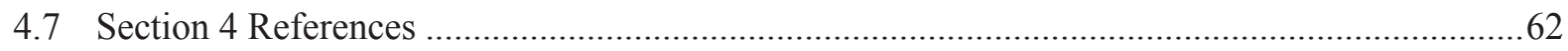




\section{TABLEOF CONTENTS}

5 Recommendations for Pre-Planning.................................................................................63

Appendix A Considerations for the Notification Phase ............................................. A-1

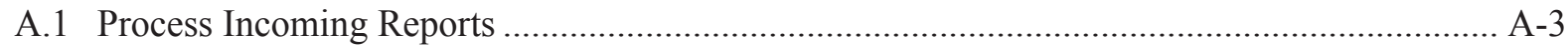

A.2 Differentiate between an Actual Attack and a Hoax ................................................................. A-3

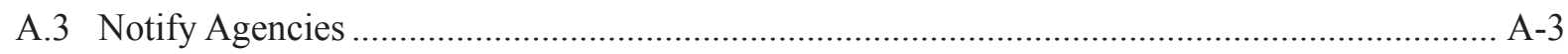

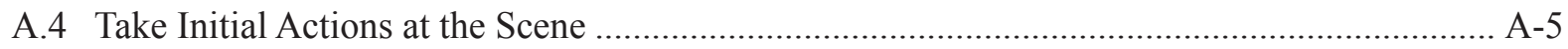

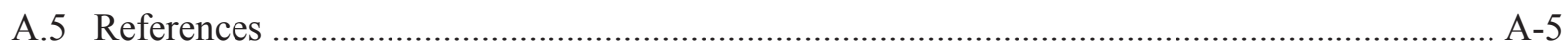

Appendix B Considerations for the First-Response Phase ................................. B-1

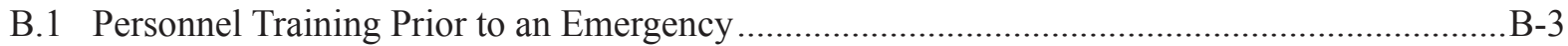

B.2 Airport First-Response Actions ......................................................................................

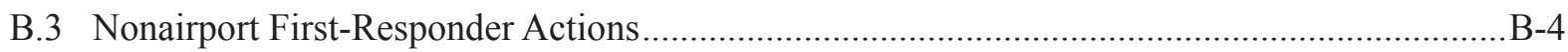

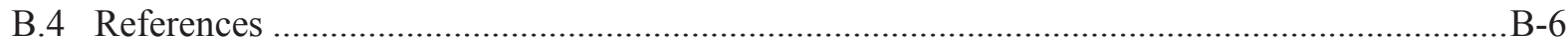

Appendix C Available Biological Sampling and Analysis Methods ................. C-1

C.1 Sample Collection Methods.............................................................................................

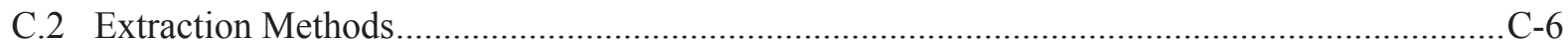

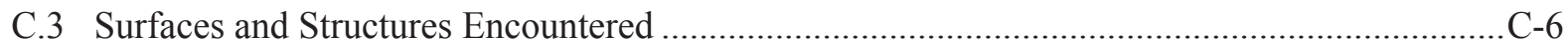

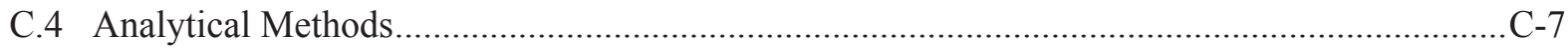

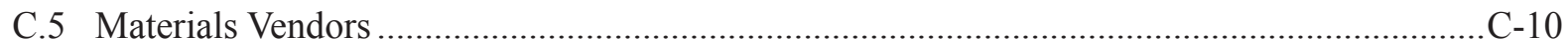

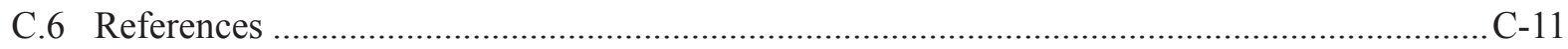

Appendix D Considerations for Sampling Design.................................................... D-1

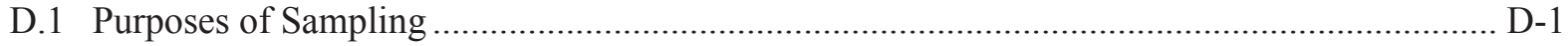

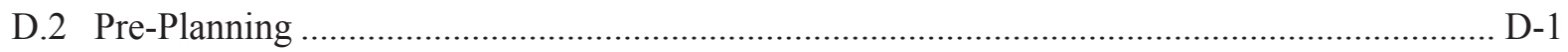

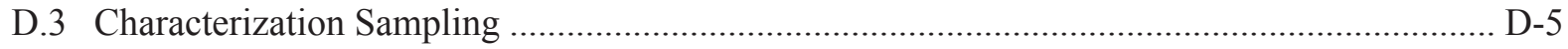

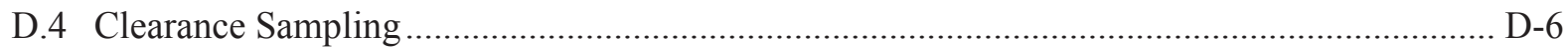

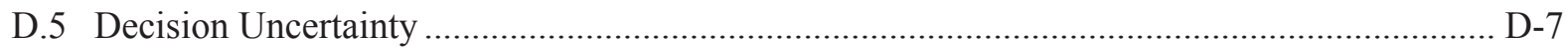

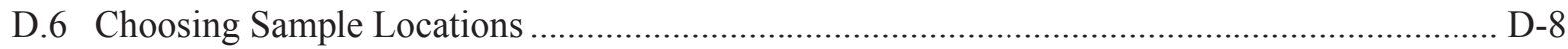

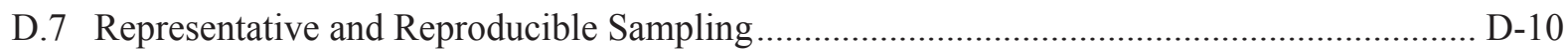

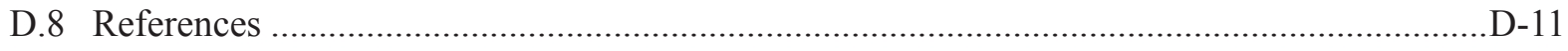

Appendix E Probability-Based Sampling ...................................................................

E.1 Applicability of Probability-Based Sampling...........................................................................

E.2 Probability-Based Sampling for Characterization ..................................................................

E.3 Probability-Based Sampling for Clearance ….......................................................................

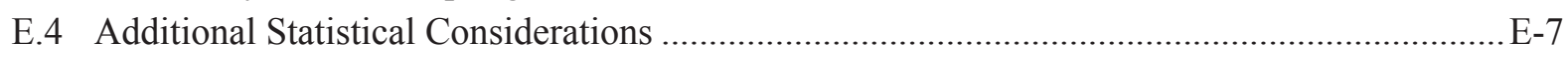

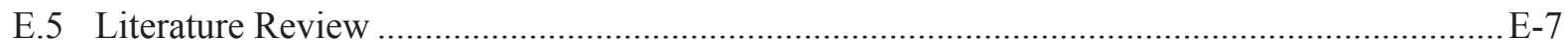

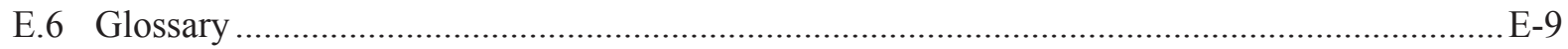

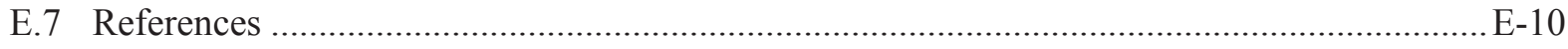

Appendix F Available Decontamination Technologies ...........................................F-1

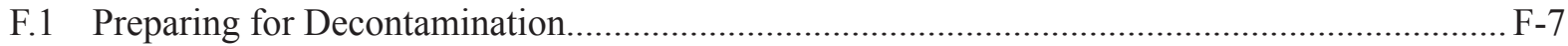

F.2 Containment and Isolation Technologies....................................................................... F-8

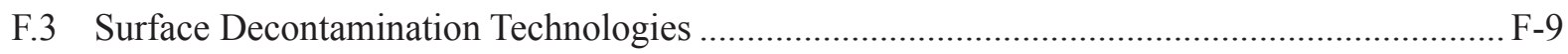




\section{TABLE OF CONTENTS}

F.4 Large-Area-Decontamination Fumigation Reagents

$\mathrm{F}-17$

F.5 Decontamination of Personal, Valuable, or Sensitive Items ............................................... F-24

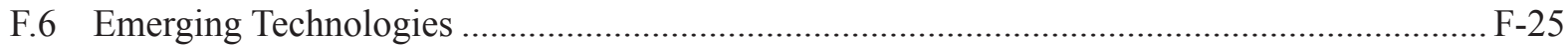

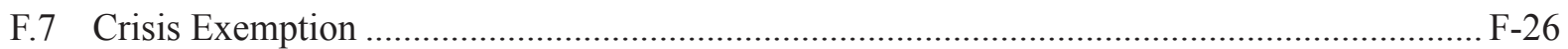

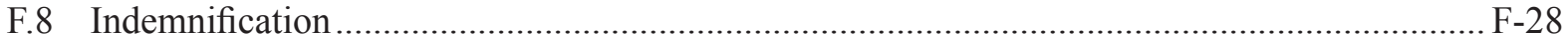

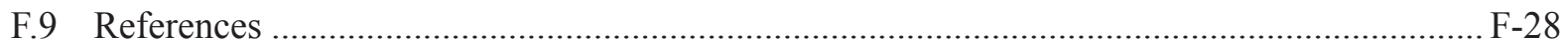

\section{Appendix G Collection, Treatment, and Disposal of}

B. anthracis-Contaminated Wastes ............................................................................. G-1

G.1 Notification of Waste and Recycling Service Providers ....................................................... G-2

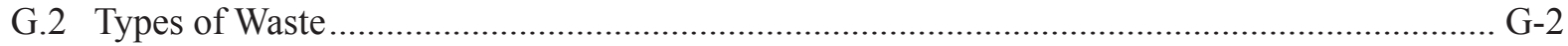

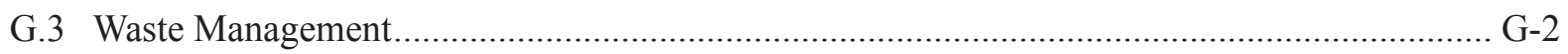

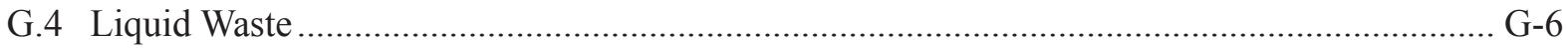

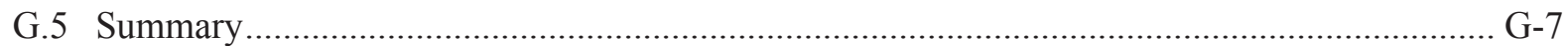

Appendix H Sampling Zone and Sampling Unit Forms

for Characterization and Clearance ............................................................................. H-1

Appendix I Annotated Characterization Sampling Plan Template ..................... I-1

Appendix J Remediation Action Plan...................................................................................... J-1

Appendix K Annotated Clearance Sampling Plan Template ................................. K-1

Appendix L Cleanup Contact List .........................................................................................-1

San Francisco International Airport Data Supplements (available separately)

A. San Francisco International Airport Restoration Concept of Operations

B. Description of San Francisco International Airport

C. Summary of HVACs/AHUs

D. Sampling Zones at San Francisco International Airport

E. Sampling Units at San Francisco International Airport

F. Remediation Action Preplanning

G. Conceptual Remediation Action Plan for use of VHP at SFO

H. Conceptual Remediation Action Plan for use of $\mathrm{ClO}_{2}$ at $\mathrm{SFO}$ 


\section{TABLEOF CONTENTS}

\section{Tables}

Table 1-1. Summary of concept-of-operations actions prior to a biological attack................ 14

Table 2-1. Site characterization resources that should be identified in advance ..................... 17

Table 2-2. Summary of characterization-related actions taken prior to a BWA attack............. 33

Table 2-3. Summary of actions during the Characterization Phase .......................................... 34

Table 3-1. Site decontamination resources .................................................................. 39

Table 3-2. Advantages and disadvantages of liquid or semi-liquid decon reagents................ 44

Table 3-3. Advantages and disadvantages of gas and vapor decontamination reagents. ......... 45

Table 3-4. Summary of decontamination-related actions prior to a BWA attack .................... 50

Table 3-5. Summary of actions during the Decontamination Phase.................................... 51

Table 4-1. Summary of clearance actions to be taken prior to a biological attack .................. 61

Table 4-2. Summary of actions during the Clearance Phase .............................................. 61

Table 5-1. Summary of actions by airport authority decision-makers prior to an attack ......... 65

Table A-1. Summary of actions to be taken before and during the Notification Phase........... A-2

Table A-2. Agencies notified if a BWA threatens health, safety, or the environment ............. A-4

Table B-1. Summary of actions to take before and during the First-Response Phase ............ B-2

Table C-1. Available collection methods for surfaces.................................................... C-7

Table E-1. Example sample numbers for discovery sampling ...............................................

Table E-2. Required sample numbers for confirmation sampling .......................................

Table F-1. Contractors experienced in decontaminating B. anthracis................................F-3

Table F-2. Aqueous-based, exposed-surface decontamination reagents ............................F-10

Table F-3. Application rates and contact times for surface decontamination reagents ..........F-15

Table F-4. Compatibility data ............................................................................ -16

Table F-5. Comparison of chlorine dioxide and vaporous hydrogen peroxide fumigants......F-23

Table L-1. Site characterization resources that should be identified in advance ......................-1

Table L-2. Site decontamination resources ................................................................2

\section{Figures}

Figure 1-1. Response phases to a biological event......................................................... 7

Figure 1-2. Range of potential scenarios following a release of BWAs ................................ 8

Figure 1-3. Plan view of SFO terminals and boarding areas ............................................. 10

Figure 1-4. Cleanup CONOPS for a $B$. anthracis release at a major airport .............................11

Figure 1-5. Components of the UC, TWG, and ECC ….............................................. 12

Figure 2-1. Actions, startup activities, and information gathering during first response........... 18

Figure 2-2. Evaluation of first-response data and steps leading to characterization .................. 21

Figure 2-3. Potential decontamination zones and characterization zones at SFO .................... 24

Figure 2-4. Evaluation of air modeling, air monitoring, and agent containment...................... 27

Figure 2-5. Major activities during the Characterization Phase ........................................... 29

Figure 3-1. Major activities during the Decontamination Phase............................................ 38

Figure 4-1. Major activities during the Clearance Phase ....................................................... 54

Figure 4-2. Developing a clearance environmental sampling plan (SAP) ............................. 58 
AAMP Ambient Air Monitoring Plan

ADM

Airport Duty Manager

AHU

air handling unit

ATSDR

Agency for Toxic Substances and Disease Registry

B. anthracis

Bacillus anthracis (sometimes referred to as B.a.)

BART

Bay Area Rapid Transit

BASIS

Biological Aerosol Sentry and Information System

BI

biological indicator

BROOM Building Restoration Operations Optimization Model

BSL

Biohazard Safety Level

BW

biological warfare

BWA

biological warfare agent

CBIAC

Chemical and Biological Defense Information Analysis Center

CBW

chemical and biological warfare

CCTV

closed-circuit television

$\mathrm{CDC}$

Centers for Disease Control and Prevention

$\mathrm{ClO}_{2}$

chlorine dioxide

CT

product of concentration (fumigant) multiplied by time

CTX

baggage-screening equipment

CWA

chemical warfare agent

DHS

Department of Homeland Security

DNA

deoxyribonucleic acid

DOD

Department of Defense

DOE

Department of Energy

DOT

Department of Transportation

ECC

Environmental Clearance Committee

EOC

Emergency Operations Center

EU

Environmental Unit

FBI

Federal Bureau of Investigation

FEMA

Federal Emergency Management Agency

FIFRA

Federal Insecticide, Fungicide, and Rodenticide Act

G agents chemical warfare nerve agents tabun, sarin, soman, and cyclosarin

GAO U.S. Government Accountability Office

HASP

Health and Safety Plan

HAZMAT hazardous materials

HEPA

high-efficiency particulate air 


\section{ACRONYMS}

HTH high-test hypochlorite (calcium hypochlorite)

HVAC heating, ventilation, and air conditioning

ICP Incident Command Post

JTTF Joint Terrorism Task Force

LBL Lawrence Berkeley Laboratory

LLNL Lawrence Livermore National Laboratory

LRN Laboratory Response Network

NAU negative air unit, also known as negative air machine, or NAM

NIOSH National Institute for Occupational Safety and Health

NIMS National Incident Management System

NRC National Research Council

NRF National Response Framework

NRT National Response Team

OSHA Occupational Safety and Health Administration

PBS phosphate-buffered solution

PCR polymerase chain reaction

PEL permissible exposure limit

POTW publicly owned treatment works

ppm parts per million

PPE personal protective equipment

QA/QC quality assurance/quality control

RAP Remediation Action Plan

SAP Sampling and Analysis Plan

SFMMTF San Francisco Metropolitan Medical Task Force

SFO San Francisco International Airport

SNL Sandia National Laboratories

TAGA Trace Atmospheric Gas Analyzer

TSA Transportation Security Administration

TWG Technical Working Group

UC Unified Command

USACE U.S. Army Corps of Engineers

USEPA U.S. Environmental Protection Agency (also referred to as EPA)

UV ultraviolet (light)

VHP vaporous hydrogen peroxide 


\section{GLOSSARY}

Ambient Air Monitoring Plan (AAMP). A written plan required by the U.S. Environmental Protection Agency as part of the crisis exemption process for using an unregistered sterilant or pesticide, such as a gaseous fumigant. The plan for monitoring ambient air is designed to ensure that the fumigant does not escape a facility in concentrations that may be a hazard to the surrounding population. (See also Crisis exemption.)

Antimicrobial agent. Any agent that kills or suppresses the growth of microorganisms. (Block 2001)

Area Command (Unified Area Command). An organization established (1) to oversee the management of multiple incidents that are each being handled by an ICS organization or (2) to oversee the management of large or multiple incidents to which several Incident Management Teams have been assigned. Area Command becomes Unified Area Command when incidents are multi-jurisdictional. Area Command may be established at an Emergency Operations Center (see EOC) facility or at some location other than an Incident Command Post (see ICP). (DHS, March 2008.)

Anthrax. A zoonotic disease caused by the spore-forming bacterium, Bacillus anthracis, and occurring in humans in three clinical forms: inhalational, gastrointestinal, and cutaneous. Inhalational anthrax results from aerosolization of Bacillus anthracis spores and is the focus of this document.

Bacillus anthracis (B. anthracis). A spore-forming bacterium that causes anthrax. The spore form is approximately $1 \mu \mathrm{m}$ wide by $2 \mu \mathrm{m}$ long and can easily be inhaled. In a warm, moist environment (such as the lungs), spores grow into vegetative, rod-shaped cells that multiply and cause hemorrhage, edema, and necrosis in humans and animals. The CDC identifies B. anthracis as a Category A (high-priority) biological agent because it can be easily disseminated, results in high mortality rates, has the potential for major public health impacts, and requires special action for public health preparedness.

BASIS Program. An environmental monitoring system originally designed for use at the 2002 Winter Olympics. Biological Aerosol Sentry and Information System (BASIS) is a joint project of two U.S. national laboratories, with participation of law enforcement and public health organizations. It uses a network of inexpensive sampling stations to collect and store aerosol samples. Couriers regularly bring samples to a central laboratory where they are analyzed for selected pathogens. Similar monitoring systems have been developed and deployed by the U.S. Department of Defense. (Fitch et al. 2003.)

Biohazard Safety Level (BSL). Different biosafety levels developed for microbiological and biomedical laboratories that provide increasing levels of personnel and environmental protection. Accordingly, laboratories may be classified as BSL-1, BSL-2, BSL-3 or BSL-4, ranked from lowest to highest in degree of safety level.

Biological event. A natural or human-caused incident involving microbiological organisms (bacteria, fungi, and viruses) or biologically derived toxins that pose a hazard to humans, animals, or plants.

Biological indicator (BI). A standardized preparation of nonhazardous bacterial spores on or in a carrier serving to demonstrate whether sterilizing conditions have been met. Spores of different organisms are used for different methods of sterilization. (Block, 2001) Also known as spore strips or spore discs, BIs consist of a known number of surrogate microorganisms (typically $10^{6}$ spores) affixed to filter paper strips or discs of stainless steel placed inside a glassine or Tyvex bag. Subsequent growth or failure of the microorganisms to grow under suitable conditions indicates the failure or adequacy of sterilization, respectively.

Biological warfare agent (BWA). Microbiological organisms (bacteria, fungi, and viruses) or biologically derived toxins that are intentionally introduced to cause disease or harm in humans, animal, or plants. Per Title 18 USC $\$ 178$, any biological material capable of causing death, disease, or other biological 


\section{GLOSSARY}

malfunction in a human, animal, plant, or another living organism; or causing deterioration of food, water, equipment, supplies, or material of any kind; or causing harmful alteration of the environment.

BioWatch Program. BioWatch is an early-warning system that can rapidly detect trace amounts of biological materials in the air whether arising from intentional release or minute quantities that are naturally in the air. The system is patterned after the Biological Aerosol Sentry and Information System (BASIS) environmental monitoring system. It operates nationwide and focuses on major urban centers. See also BASIS. (DHS BioWatch web site and Fitch et al. 2003.)

Characterization. The process of obtaining specific information about a biological event for the purpose of determining further action. The focus is on determining key site parameters associated with the location(s) of concern, time since release, extent of contamination, and related data. Characterization includes obtaining information on the identity, genetic composition, formulation, physical properties, and toxicological properties of the biological agent; identifying the locations or items contaminated, amount of contamination, ability of the agent to aerosolize, and persistence of the agent; and estimating the potential for exposure to the agent. Characterization generally occurs after the First-Response Phase and before the Decontamination Phase (see Figure 1-1).

Characterization sampling. Environmental sampling intended to assess the nature (identity and properties) and extent (location and quantity) of contamination of an area or items, and to provide information needed to decide where to decontaminate, what to decontaminate, and how to decontaminate. Generally occurs after the First-Response Phase and before the Decontamination Phase (see Figure 1-1).

Characterization zone. A discrete section or segment of a contaminated site, such as the first floor of a particular terminal, that is a manageable piece for gathering data related to characterization.

Chemical warfare agent (CWA). A gaseous, liquid, or chemical substance intended for military use (or used by terrorists in the context of this document) with lethal or incapacitating effects on personnel through its chemical properties. The classes of chemical agents commonly used for chemical warfare and terrorism are (1) nerve agents, (2) blister agents, and (3) choking agents, all of which produce incapacitation, serious injury, or death. (Modified from the Center for Nonproliferation Studies at the Monterey Institute of International Studies, Glossary, Biological Weapons Terrorism Tutorial, 2004.)

Cleanup. The process of characterizing, decontaminating, and clearing a contaminated site or items. Generally occurs after the First-Response Phase and before the Restoration Phase (see Figure 1-1). Used in this document as a synonym for remediation.

Cleanup goal. An amount of residual contamination, or process, established for a specific contaminant that provides acceptable protection to human health and the environment once that amount or process is achieved. If the cleanup goal is established for an area, it specifies criteria for determining the success of decontamination that are measurable and that must be met to permit unprotected reentry to the area. A cleanup goal may also be established for items, such as equipment or personal belongings.

Clearance. The process of determining that a cleanup goal has been met for a specific contaminant at a specific site or on an item. Clearance generally occurs after decontamination and before restoration. Clearance typically includes environmental sampling together with analysis of data by subject-matter experts (such as an Environmental Clearance Committee) and stakeholders to ensure that all long-term health and environmental issues are addressed.

Clearance sampling. Environmental sampling conducted after decontamination that is intended to provide a basis for determining whether a cleanup goal is met for a specific contaminant in an area or on items. 


\section{GLOSSARY}

Clearance zone. A discrete section or subsection of a contaminated site for which a clearance decision is made.

Cold zone. See staging area.

Concept of Operations (CONOPS). A formal plan that describes the roles, responsibilities, and relations of organizations involved in a response to a contaminated area or items. A CONOPS typically addresses Federal, state, and local agencies and how they should interact when responding to a potential or actual terrorist threat or incident.

Containment. In the context of this document, includes actions or measures taken to prevent the spread of a contaminant from a particular zone or to prevent the movement of a contaminant within a zone. Compare with Isolation. This term is defined differently by different agencies.

Contaminant reduction zone. The transition area between the exclusion and support zones where responders enter and exit the exclusion zone and where decontamination activities of responders take place. Also called the Warm Zone. (USEPA 2004.)

Covert release. In the context of this document, includes the intentional release of a biological warfare agent that is not reported or openly acknowledged by terrorists, or observed by surveillance systems, or witnessed by potential victims at the scene of the release, and typically requiring epidemiological or medical observations to lead to the discovery of an agent's release.

Crisis exemption. A mechanism by which a state or Federal agency (such as the USEPA), under the authority of Section 18 of the Federal Insecticide, Fungicide, and Rodenticide Act (FIFRA), may exempt a pesticide product from the requirement for registration, and temporarily permit the use of an unregistered pesticide. For example, the USEPA issued crisis exemptions for antimicrobial pesticides used to inactivate Bacillus anthracis spores at sites contaminated from the mail attacks in late 2001.

Culturing. In this document, growing microorganisms in a controlled, artificial environment. The CDC bacterial culture method is the definitive confirmatory test on environmental samples for positive identification of Bacillus anthracis spores upon which public health decisions are made.

Decision maker. A person charged with determining and directing appropriate actions in response to a potential or actual biological event at a particular site.

Decontamination. The process, including natural attenuation, of inactivating or reducing a contaminant in or on buildings, humans, animals, plants, food, water, soil, air, areas, or other items through physical, chemical, or other methods to meet a cleanup goal. Decontamination generally occurs after characterization. This term is defined differently by Federal agencies and other entities. For the purposes of this document, decontamination includes waste disposal.

Decontamination area or zone. A discrete section or subsection of a contaminated site that can be subjected to containment with respect to other areas and then decontaminated as a unit.

Decontamination reagent. A substance that is used to inactivate or reduce a contaminant on humans, animals, plants, inanimate surfaces, or in other media. If the contaminant is a microorganism, the reagent may be an antimicrobial pesticide.

Disinfectant. A chemical or physical agent that destroys pathogenic or other harmful microorganisms, but not bacterial spores on inanimate surfaces.

Disposal. The deposition or placement of any solid or hazardous waste on or in the land or water. Disposal of wastes from the remediation of airport facilities will likely be accomplished through the use of permitted/ licensed landfills meeting certain required criteria (i.e., if the material being disposed is a hazardous waste, 


\section{GIOSSARY}

it would be sent to a hazardous waste landfill) or through appropriate treatment technologies such as steam autoclaving or incineration. It is important to note that treatment technologies such as incineration may generate residues which must be tested and then appropriately disposed, most often in either a hazardous waste or a municipal solid waste landfill.

Emergency Operations Center (EOC). The physical location at which the coordination of information and resources to support domestic incident management activities normally takes place. An EOC may be a temporary facility or may be located in a more central or permanently established facility, perhaps at a higher level of organization within a jurisdiction. EOCs may be organized by major functional disciplines (e.g., fire, law enforcement, and medical services), by jurisdiction (e.g., Federal, state, regional, county, city, or tribal), or by some combination thereof. (DHS, March 2008.)

Environmental Clearance Committee (ECC). An independent group of scientific experts from a variety of Federal, state, and local agencies that provides advice, data and process analysis, and recommendations related to decontamination of a facility. An ECC provides a final recommendation on whether the cleanup was adequate to justify reopening a facility for normal operations and use. Although not required, the use of an ECC to evaluate the adequacy of cleanup through highly qualified professional debate is recommended to ensure adequate protection of public health. (Modified from Proceedings from the 2nd Civilian-Military Anthrax Response Technical Workshop, 2004.)

Environmental sampling. Sampling for a biological agent conducted on inanimate surfaces or in air, water, or soil. In the context of this document, includes the collection of indoor samples to determine the presence of Bacillus anthracis spores and the extent and degree of contamination, assess risk of exposure, support decisions regarding the need for medical treatment or cleanup, and provide guidance about when cleanup is adequate to permit reoccupancy.

Environmental Unit. An Incident Command System unit in the Panning Section responsible for environmental matters associated with a response, including strategic assessment, modeling, and environmental monitoring and permitting.

Exclusion zone. An area with actual or potential contamination and the highest potential for exposure to the contaminant. Entry to the exclusion zone is permitted only for persons wearing appropriate personal protective equipment (PPE). Equivalent to hot zone, red zone, or restricted zone.

First responders. Primarily police, fire, and emergency personnel who in the early stages of an incident are responsible for the protection and preservation of life, property, evidence, and the environment, including emergency response providers as defined in Section 2 of the Homeland Security Act of 2002 (6 U.S.C. 101), as well as emergency management, public health, clinical care, public works, and other skilled personnel who provide immediate support services.

First response. Actions taken immediately following notification of a biological event or release. In addition to search and rescue, scene control, and law-enforcement activities, first response includes initial site containment, screening sampling and analysis, and personnel decontamination. Follows the Notification Phase of a response (see Figure 1-1).

Federal On-Scene Coordinator (FOSC OR OSC). The Federal official predesignated by the USEPA or the USCG to coordinate responses under subpart D of the National Contingency Plan (NCP); or the government official designated to coordinate and direct removal actions under subpart E of the NCP. (DHS, March 2008.)

Fumigant. In the context of this Remediation Guidance document, a fumigant is a gaseous or vaporized decontamination reagent, such as vaporous hydrogen peroxide, chlorine dioxide, ethylene oxide, methyl 


\section{GLOSSARY}

bromide, or paraformaldehyde, which is known to be effective in killing Bacillus anthracis spores and used during cleanup.

Fumigation. Use of a chemical gas or vapor in a contained space to inactivate biological contaminants, primarily pathogenic bacteria, fungi, and viruses.

Fumigation zone. A discrete section or subsection of a building or facility that is isolated with respect to other areas of the building or facility for the purposes of fumigation. See Isolation.

Hazardous material. A substance or material, including a hazardous substance, that has been determined by the Secretary of Transportation to be capable of posing an unreasonable risk to health, safety, and property when transported in commerce, and which has been so designated (49 CFR 171.8).

Health and Safety Plan (HASP). A written plan required under the Occupational Health and Safety Administration's (OSHA's) Hazardous Waste Operations and Emergency Response (HAZWOPER) standard (29 CFR 1910.120). This standard requires a written HASP, which identifies site hazards and appropriate controls to protect employee health and safety. (National Response Team, NRT 2005.) The HASP describes known physical, chemical, and biological hazards at a site; the establishment of hot (contaminated), cold (uncontaminated), and warm (intermediate) zones; personal protective equipment (PPE); personal decontamination procedures; and emergency procedures to be used by sampling and decontamination personnel.

Hot zone. See Exclusion zone.

Inactivation. Removing the activity of microorganisms by killing or inhibiting reproductive or enzyme activity. When referring to an antimicrobial agent, inactivation means neutralizing the activity of microorganisms by any means. (Block 2001.)

Incident. An occurrence or event, natural or human-caused, that requires an emergency response to protect life or property. Incidents can include major disasters, emergencies, terrorist attacks, terrorist threats, wild land and urban fires, floods, hazardous materials spills, nuclear accidents, aircraft accidents, earthquakes, hurricanes, tornadoes, tropical storms, war-related disasters, public health and medical emergencies, and other occurrences requiring an emergency response. (DHS, March 2008.)

Incident Action Plan (IAP). An oral or written plan containing general objectives reflecting the overall strategy for managing an incident. It may include the identification of operational resources and assignments. It may also include attachments that provide direction and important information for managing the incident during one or more operational periods. In the context of this document, the RAP (see Remediation Action Plan) is implemented through a series of IAPs.

Incident Commander (IC). The individual responsible for all incident activities, including the development of strategies and tactics and the ordering and the release of resources. The IC has overall authority and responsibility for conducting incident operations and is responsible for the management of all incident operations at the incident site. (NIMS, March 2004; DHS, March 2008.)

Incident Command Post (ICP). As defined in the NRF, the ICP is the field location at which the primary tactical-level, on-scene incident command functions are performed. The ICP may be collocated with the incident base or other incident facilities and is normally identified by a green rotating or flashing light. Compare with EOC.

Incident Command System (ICS). A standardized, on-scene, emergency management construct specifically designated to provide for the adoption of an integrated organizational structure that reflects the complexity and demands of single or multiple incidents, without being hindered by jurisdictional 


\section{GIOSSARY}

boundaries. ICS is the combination of facilities, equipment, personnel, procedures, and communications operating with a common organizational structure, designed to aid in managing resources during incidents.

Infectious dose (ID). A dose at which an organism can reproduce in the host and produce a measurable effect. (Johnson 2003.)

Isolation. For the purposes of this document, action taken to seal a site to permit fumigation and prevent release of fumigant. Compare with containment. This term has been used differently by various agencies.

Laboratory Response Network (LRN). The organization of public health laboratories established by the Department of Health and Human Services, Centers for Disease Control and Prevention (CDC) in accordance with Presidential Decision Directive 39. The LRN and its partners maintain an integrated national and international network of laboratories that are equipped to respond quickly to acts of chemical or biological terrorism, emerging infectious diseases, and other public health threats and emergencies. (CDC, 2005.) In a partnership of the CDC, FBI, and Association of Public Health Laboratories, the LRN fulfills the Federal responsibility of rapid sample testing and identification of biological and chemical threat agents through established protocols and reagents. The LRN also serves as a sentinel warning system for covert biological events.

Life-safety zones. The interior zones or regions of a building that are used for smoke control in the event of a fire. Life-safety zones are defined by the dedicated air-handling units (AHUs) of the building's heating, ventilation, and air conditioning (HVAC) system. They constitute logical zones for characterization and decontamination.

Natural attenuation. The degradation of a hazardous substance, including biological warfare agents, into less hazardous forms via natural, environmental mechanisms such as heat, light, or chemical reactions.

Negative air unit (NAU). A system that subjects an area to a slightly negative pressure to ensure that a contaminant (and decontamination reagent) remains in the contamination zone. NAUs consist of a HEPA filter, chemical scrubber, demister, carbon bed, fan, and stack. Air within a building is exhausted through HEPA filters at a rate sufficient to pull a slightly negative pressure in the contaminated zone.

Notification. The process of communicating the occurrence or potential occurrence of a biological event to and through designated authorities who will initiate first-response actions. Notification generally occurs as the first step in response to a biological event (see Figure 1-1).

National Incident Management System (NIMS). A nationwide template enabling Federal, state, local, and tribal governments and private-sector and nongovernmental organizations to work together effectively and efficiently to prevent, prepare for, respond to, and recover from domestic incidents regardless of cause, size, or complexity. The NIMS provides a core set of doctrine, concepts, terminology, and organizational processes to enable collaborative incident management at all levels.

National Response Framework (NRF). An all-discipline, all-hazards plan that establishes a single, comprehensive framework for managing domestic incidents. The NRF provides the structure and mechanisms for coordinating Federal support and exercising direct Federal authorities and responsibilities.

Operations Section. The ICS section responsible for all tactical incident operations.

Overt release. In the context of this document, the intentional release of a biological warfare agent that is reported or openly acknowledged by terrorists, or observed by surveillance systems, or witnessed by potential victims at the scene of the release, or made known at the time of release by other means.

Pathogen. Any disease-producing microorganism. (Block 2001.) 


\section{GLOSSARY}

Planning Section. The Incident Command System section responsible for collecting, evaluating, and disseminating operational information related to an incident and for preparing the Incident Action Plan. The Planning Section maintains information on the current and forecasted situation and on the status of resources assigned to the incident.

Polymerase chain reaction (PCR). A method, sometimes referred to as "molecular photocopying," for generating copies of a fragment of DNA. PCR can characterize and synthesize any specific piece of DNA, and identify genetic material from specimens, including microbes, such as Bacillus anthracis. As a field test described in this document, the rapid, automated, and quantitative PCR technique involves a portable piece of equipment using a reaction tube, reagents, and a heat source to obtain presumptive evidence of the presence of Bacillus anthracis spores. Confirmation of the presence of such spores must be obtained through the laboratory culture of sampled material, a more lengthy process.

Principal Federal Official (PFO). The Federal official designated by the Secretary of Homeland Security to act as his/her representative locally to oversee, coordinate, and execute the Secretary's incident management responsibilities under HSPD-5 for major incidents. (DHS, March 2008.)

Process monitoring. Measuring and recording the key variables of a decontamination process as they occur. For example, during fumigation, the key variables of fumigant concentration, contact time, temperature, and relative humidity are measured and documented over time.

Quality Assurance. An integrated system of activities involving planning, quality control, quality assessment, reporting, and quality improvement to ensure that a product or service meets defined standards of quality with a stated level of confidence. (USEPA 2002.)

Quality Control. The overall system of technical activities the purpose of which is to measure and control the quality of a product or service so that it meets the needs of its users. The aim is to provide quality that is satisfactory, adequate, dependable, and economical. (USEPA 2002.)

Recommissioning. The process of testing and verifying that equipment and systems are fully functional and may be returned to normal use. Recommissioning can include buildings as well.

Recovery. In the short term, recovery is an extension of the response phase in which basic services and functions are restored. In the long term, recovery is a restoration of both the personal lives of individuals and the livelihood of the community. Recovery can include the development, coordination, and execution of service- and site-restoration plans; the reconstitution of government operations and services; programs to provide housing and to promote restoration; long-term care and treatment of affected individuals; and additional measures for social, political, environmental, and economic restoration. (DHS, 2008).

Remediation. The process of characterizing, decontaminating, and clearing a contaminated site or items, including disposal of wastes. Generally occurs after the First-Response Phase and before the Restoration Phase (see Figure 1-1). A synonym for cleanup.

Remediation Action Plan (RAP). A formal plan developed for the Incident Commander that describes actions to remove, reduce, or eliminate contaminants at a site. The RAP is developed at the beginning of the Decontamination Phase. In the context of this Remediation Guidance document, the RAP is a written, incident-specific plan that includes details on (1) what facilities and areas need to be decontaminated; (2) what materials and structural components are to be decontaminated in situ, or removed for treatment and either reuse or disposed; (3) to what extent removed items will be decontaminated prior to disposal, and how and where such items will be decontaminated and disposed; (4) the decontamination technologies to be used; (5) the personnel and teams responsible for 


\section{GLOSSARY}

decontamination tasks; and (6) the types of wastes that will be produced and how they will be treated or disposed.

Renovation. The process of reconstructing or refurbishing a facility before allowing occupants to return.

Residual contamination. Any amount of contaminant remaining after an area has been decontaminated.

Response. Includes immediate actions to save lives, protect property and the environment, and meet basic human needs. Reesponse also oncludes the execution of emergency plans and actions to support short-term recovery (DHS, 2008).

Restoration. The process of renovating or refurbishing a facility, bringing it back to an unimpaired or improved condition, and making a decision to allow the occupants to return. Generally occurs after the Clearance Phase but before occupants are allowed to return (see Figure 1-1).

Sampling and Analysis Plan (SAP). A plan that describes the methods, strategies, and analyses for characterization environmental sampling, verification sampling (if applicable), and clearance sampling for a contaminated site.

Sampling unit. A subsection of a sampling zone, such as walls, floors, and furniture surfaces, that can be sampled and evaluated collectively.

Sampling zone. A discrete section of a contaminated site in which environmental sampling is conducted.

Screening analysis. The process of analyzing environmental samples by nonLRN personnel, equipment, or facilities is considered to constitute "first-pass," "screening," or "field" testing. There is no recognized, definitive, reliable field test for biological agents. Compare with Laboratory Response Network and Culturing, above.

Screening sampling. Collecting environmental samples during the first response to a biological event. Same as initial environmental sampling.

Source reduction. In the context of this Remediation Guidance document, activities designed to meet the objective of decreasing the biological agent within a contaminated facility prior to the main decontamination activities, such as extensive fumigation. Source reduction can include the removal of material and items from a contaminated building to make decontamination easier.

Spores. The thick-walled, resting cells produced by some bacteria and fungi that are capable of surviving in unfavorable environments and are more resistant to antimicrobial agents than vegetative cells. (Block 2001.)

Staging area. A safety zone established at a hazardous substance release site that is designated as the support zone (or cold zone). It is the area of the site that is free from contamination and that may be safely used as a planning and staging area. (USEPA 2004.)

Sterilant. A substance that destroys all microorganisms on inanimate surfaces, including vegetative and spore forms of bacteria and fungi, as well as viruses. Sterilants registered by the USEPA must be effective on both porous and nonporous surfaces.

Sterilization. A process intended to remove or destroy all viable forms of microbial life, including bacterial spores, to achieve an acceptable sterility assurance level. (AAMI 1995.)

Subject-matter expert (SME). An individual who is a technical expert in a specific area or in performing a specialized job, task, or skill.

Support Zone. The area of the site that is free from contamination and that may be safely used as a planning and staging area. (USEPA 2004.) See also staging area. 


\section{GLOSSARY}

Swab sampling. Collecting environmental samples by rubbing a small area on nonporous surfaces with a dry or wet absorptive material attached to the end of a wood or plastic stick.

Technical Working Group (TWG). A group of technical experts assembled by the Unified Command to provide guidance during the planning and implementation of cleanup operations.

Unified Command. An application of the Incident Command System used when there is more than one agency with incident jurisdiction or when incidents cross political jurisdictions. Agencies work together through the designated members of the Unified Command to establish their designated Incident Commander at a single Incident Command Post (ICP) and to establish a common set of objectives and strategies and a single Incident Action Plan (IAP). (DHS, March 2008.) In the context of this document, the IAP is equivalent to the RAP (see Remediation Action Plan).

Vacuum sampling. Collecting environmental samples by suction on porous or nonporous surfaces with a vacuum cleaner that contains a high-efficiency particulate air (HEPA) filter.

Vegetative cells. Microbial cells in the growth and reproductive phase of a growth cycle. (Block 2001.)

Verification sampling. Use of chemical or biological indicators, or both, to document that fumigation decontamination processes have been successful. The term "decontamination verification" is sometimes used to include meeting the fumigation parameters as well as use of chemical or biological indicators to document success.

Warm zone. Transition area between the exclusion and support zones, where responders enter and exit the exclusion zone, and where decontamination activities take place. (USEPA 2004.)

Weapon of mass destruction (WMD). As defined in Title 18, U.S.C. § 2332a: (1) any explosive, incendiary, or poison gas, bomb, grenade, rocket having a propellant charge of more than 4 ounces, or missile having an explosive or incendiary charge of more the one-quarter ounce, or mine or similar device; (2) any weapon that is designed or intended to cause death or serious bodily injury through the release, dissemination, or impact of toxic or poisonous chemicals or the precursors; (3) any weapon involving a disease organism; or (4) any weapon that is designed to release radiation or radioactivity at a level dangerous to human life.

Wipe sampling. Collecting environmental samples by rubbing a small area on nonporous surfaces with a thin, flat piece of dry or wet absorptive material. 


\section{GLOSSARY}

\section{Glossary References}

AAMI (1995), Association for Advancement of Medical Instrumentation, Standards and Recommended Practices. Sterilization, Part I. Sterilization of Health Care Facilities; Sterilization, Part 2. Hospital Equipment and Industrial Process Control, Arlington, VA.

Block, S. (Ed.) (2001), Disinfection, Sterilization, and Preservation, $5^{\text {th }}$ Edition (Lippincott, Williams, and Wilkins, Philadelphia, PA).

CDC (2005), Centers for Disease Control and Prevention, The Laboratory Response Network; available at http://www.bt.cdc.gov/lrn/

DHS (March 2008), Department of Homeland Security, National Response Framework; available at http:// www.fema.gov/emergency/nrf/

Fitch, J. P., Raber, E., and D. R. Imbro (2003), "Technology Challenges in Responding to Biological or Chemical Attacks in the Civilian Sector," Science 302(5649), 1350-54; see also Fitch, J. P. et al., Proc. IEEE 90(11), 1708 (2002).

Johnson, B. (2003), “OSHA Infectious Dose White Paper,” Applied Biosafety 8(4), 160-165.

NIMS (March 1, 2004), National Incident Management System, document available from FEMA at 1-800480-2520, Option 4, ask for FEMA 501.

NRT (updated July, 2005), National Response Team, Technical Assistance for Anthrax Response, InterimFinal Draft; available only by electronic means.

Proceedings from the 2nd Civilian-Military Anthrax Response Technical Workshop (April 13-14, 2004), "Current State of Federal Governmental Capabilities and Positions;" available at http://www.wetp.org/wetp/ public/hasl_get_blob.cfm?ID=741

USEPA (2002), U.S. Environmental Protection Agency, Health Effects Notebook, Health Effects Glossary; available at http://www.epa.gov/ttnatw01/hlthef/hapsec1.html

USEPA (2004), U.S. Environmental Protection Agency, Safety Zones, Emergency Response Programs; available at http://www.epa.gov/superfund/programs/er/hazsubs/safety.htm 
This document provides general guidelines for airport managers, local officials, and other stakeholders for developing a response and recovery plan for a major airport following the release of a biological warfare agent, with specific guidance for remediation. San Francisco International Airport was selected as the example airport during development of the guidance to illustrate specific details. The spore-forming bacterium Bacillus anthracis was selected as the biological agent of primary concern because it is the most difficult of known bioterrorism agents to inactivate and is considered to be one of the agents most likely to be used as a biological weapon. The focus of the guidance is on cleanup and disposal activities associated with the Characterization, Decontamination, and Clearance Phases that are defined herein. Activities associated with the Notification and First-Response Phases are briefly discussed in Appendixes A and B, respectively. In addition to the main text of this Remediation Guidance document and associated appendixes, a data supplement was developed specifically for San Francisco International Airport. Requests for the data supplement must be made directly to the Emergency Planning Operations Division of San Francisco International Airport.

This document does not describe public health responses to release of a biological warfare agent. If laboratory analytical results confirm the presence of a biological agent, the responsible public health agency involved in the response will commence appropriate public health actions, such as treatment (CDC 2004) and decontamination of potentially contaminated individuals, distribution of prophylaxis, and medical examinations. See the Centers for Disease Control and Prevention web site for more information on emergency public health responses (http://www.bt.cdc.gov/).

Effective communication with the media and the public is an essential component of airport remediation and recovery. Whereas such communication is beyond the scope of this document, many helpful resources are available. (See Section 1.1 for more information.)

The concept of operations described in this document for cleanup of a Bacillus anthracis release at a major airport conforms to the National Response Framework (NRF) (DHS 2008) and implementation of the National Incident Management System (NIMS 2004). In most cases, a Unified Command would be formed to direct the cleanup process jointly and to take ultimate responsibility for all cleanup decisions. The Unified Command would likely include the Airport Manager or Airport Emergency Operations Manager, representatives from state and local public health and emergency management agencies, and Federal agencies, such as the U.S. Environmental Protection Agency. The Unified Command and Incident Command Staff would ideally be co-located in an Incident Command Post in an uncontaminated area of the airport. If a release is large or complex, co-location of all key players may not be possible. If a major incident is declared, a Principal Federal Official may be appointed by the Department of Homeland Security to facilitate Federal support to the Unified Command. In some facilities and jurisdictions, additional response plans or procedures (such as BioWatch or other emergency response plans, procedures, or protocols) may govern notification and first response. Facility personnel, responders, and emergency management coordinators should be aware of all applicable plans and procedures and how to implement them.

Cleanups following the anthrax attacks of 2001 in the U.S. pre-dated the NRF and NIMS and did not make use of a NIMS Incident Command System as we know it today. Most Incident Commanders for the larger of those cleanups chose to convene a Technical Working Group (TWG) of multi-agency, multi-disciplinary, outside experts to advise in developing sampling and decontamination plans. Most of the Incident Commanders also convened a separate Environmental Clearance Committee (ECC) to independently review pre- and postdecontamination sampling data as well as data on decontamination parameters, to evaluate whether the decontamination was effective, and to add credibility regarding a decision that decontaminated areas may be reoccupied. Use of such specialized technical expertise is strongly recommended, although it would now operate within the construct of the NIMS Incident Command System. The TWG, if convened, would act in an 


\section{OVERVIEW}

advisory capacity to the Environmental Unit within the Planning Section. Likewise, the ECC, if convened, would provide independent peer review of products and recommendations by the Environmental Unit. For maximum impartiality, the ECC could also provide recommendations directly to the Unified Command.

Cleanup activities commence with site characterization. The principal goal of characterization is to define the extent of contamination and to gather information needed to design the decontamination approach. Initial sampling data collected by first responders are assessed to approximate the location(s) of contamination. Confirmation of the agent type and viability is obtained from a Laboratory Response Network laboratory. Additional characterization data are collected to determine the extent of contamination and where decontamination actions are needed. Areas suspected of being contaminated are contained and isolated to the degree possible to prevent further movement of an agent to uncontaminated areas or the environment, and to reduce the potential for future exposure from the agent or fumigant, if fumigation is needed. Agent air monitoring in areas adjacent to the contained contamination zones is done to ensure the protection of remediation personnel and to monitor any release from the contained zones. Various sampling approaches are considered. For example, wipe samples can be used to sample hard, nonporous surfaces. Swab samples can be used to sample nooks, crannies, joints, and seams. Appendix I is a template for preparing an incident-specific, operational characterization plan. Upon completion of the characterization plan, an internal review is initiated. Upon approval of the plan by the Unified Command, characterization commences.

The Site Safety Officer develops a Health and Safety Plan to ensure that coordinated health and safety measures are in place for all responding personnel. This plan describes physical, chemical, and biological hazards at the site, personal protective equipment, personal decontamination procedures, and emergency procedures to be used by sampling and remediation personnel.

An incident-specific Remediation Action Plan is developed, which describes the decontamination methods to be used and other details, including waste disposal. Under NIMS, this overall plan, as well as other plans described below, are implemented through a series of standardized, shorter-term Incident Action Plans, which describe specific activities that will take place during a shift or a day. The template in Appendix $J$ is designed to facilitate preparation of the Remediation Action Plan. If fumigation will be done, a Sampling and Analysis Plan will be needed, as well as an Ambient Air Monitoring Plan, which can be a component of the Remediation Action Plan. These three plans are needed to obtain a crisis exemption from the U.S. Environmental Protection Agency for authorized use of an unregistered sterilant or pesticide during cleanup. If pesticide products become registered for inactivation of $B$. anthracis in the future, the three plans will likely be required by the product's labeling, but the plans would no longer be submitted to the U.S. Environmental Protection Agency for approval; rather, the Incident Commander would approve them. If fumigation is used, an Emergency Response Plan is also recommended, although such a plan is not required for a crisis exemption.

For some nonpersistent biological warfare agents, natural attenuation may be an adequate decontamination option. In cases where contamination is not extensive or an agent is not persistent, application of surface decontaminants or other methods of medical infection control may be effective. For extensive contamination by persistent agents, such as Bacillus anthracis, fumigation may be necessary. In those cases, the Decontamination Phase commences with source reduction, such as pre-cleaning surfaces to reduce the contaminant load. To expedite cleanup and prevent costs from escalating unnecessarily, a cost-benefit analysis should be incorporated in the decision process related to retention versus disposal of facility items and materials. Certain materials and structural components can be decontaminated for reuse, but it may not be feasible or cost-effective to decontaminate other items. Those items must be pre-treated, packaged (with the package decontaminated externally), and removed for disposal as waste. 


\section{OVERVIEW}

Site preparation includes sealing openings to prevent leaks and setting up equipment. Decontamination reagents and delivery systems are selected, and pre-testing all systems is done before carrying out chemical treatment(s). The important choice of decontamination technologies depends on characteristics of the agent, the nature and extent of contamination, and other site parameters identified during characterization. Decontamination-related decisions can have a major impact on waste-disposal costs, and it is necessary to develop a disposal plan that identifies a means of disposal, necessary approvals, transportation, and other details.

A clearance strategy is developed to ensure that after decontamination, the risk of exposure to residual contamination is negligible. A clearance sampling plan is developed, which sets clearance criteria, specifies how to determine whether the criteria have been met, and describes how and where to collect clearance samples after decontamination. In most cases, clearance sampling includes both surface and aggressive air samples. Appendix $\mathrm{K}$ is a clearance plan template that identifies the types of required information.

To determine whether the decontaminated area may be reoccupied, the Environmental Unit (and the ECC separately, if one is convened) evaluates the results of clearance samples. For the anthrax incidents to date, and given methods that have been used, cleanup was considered successful only when there was no growth of B. anthracis cultured from any environmental samples taken after decontamination. Other factors that will be considered include the decontamination process parameters (e.g., temperature, relative humidity, decontaminant concentration, and contact time) and the results from post-decontamination culture of biological indicators that may have been used during fumigation or sterilization processes. From such an evaluation the Environmental Unit or ECC, or both, recommend whether the area may be reoccupied, or whether further decontamination is necessary. The UC makes the final decision that a facility is appropriately decontaminated for reoccupancy. The facility owner or responsible public health agency makes the final decision to reopen a decontaminated facility.

A theme that is emphasized throughout this document is that many activities can greatly reduce the time required to restore airport operations if those activities are conducted prior to an actual release of biological warfare agent. A summary of specific resources (such as Federal, state, and local agency contacts; contractors; prospective team members; subject-matter experts; laboratory facilities; and other entities) that should be identified in advance by airport officials is provided in each pertinent section of this document, and summary contact lists of such resources are provided in Appendix L. A summary of overall pre-planning actions that should be completed by airport officials is presented at the end of this document.

\section{Overview References}

Centers for Disease Control and Prevention (CDC) (September, 2004), Fact Sheet: Isolation and Quarantine; Fact Sheet: Legal Authorities for Isolation and Quarantine; and Questions and Answers: Legal Authorities for Isolation and Quarantine, Department of Health and Human Services, Centers for Disease Control and Prevention.

DHS (March 2008), Department of Homeland Security, National Response Framework; available at http://www.fema.gov/emergency/nrf/.

NIMS (March 1, 2004), National Incident Management System, document available from FEMA at 1-800-480-2520, Option 4, ask for FEMA 501. 
Airport Biological Remediation Guidance 4 


\section{INTRODUCTION}

\section{Introduction}

In the event of a terrorist attack involving the release of a biological warfare agent (BWA) at a major airport, decision makers will need to make important choices about how to respond. This Remediation Guidance document identifies key activities and issues that must be considered by a typical major airport following an incident involving release of a BWA. Appendixes provide more detailed information on key topics as well as templates that should be completed for a particular facility.

\subsection{Description of Phases}

Actions following a terrorist incident can be categorized into six principal phases, beginning with identification of an incident and ending with verification that all cleanup and decontamination criteria have been met (Raber et al. 2002) followed by reoccupancy of a site. The six phases are shown in Figure 1-1 and can be briefly characterized as follows:

- Notification Phase. When evidence of a biological incident surfaces, airport management or its Emergency Operations Center (EOC) receives and assesses that information, identifies the potential release sites, and notifies all appropriate agencies of the incident.

- First-Response Phase. Central concerns are containing the area of contamination and the crime scene; rescuing, evacuating, and, if needed, quarantining (DOT 2006) or otherwise managing affected persons; mitigating any conditions that pose an immediate threat to human health (such as fire or explosion); and minimizing the spread of contamination. This phase begins with activation of an Incident Command and of law enforcement and emergency operations personnel [e.g., security, medical, and hazardous materials (HAZMAT) teams, as needed] and continues as long as emergency personnel are present. The phase ends when conditions immediately dangerous to human health are brought under control and when law enforcement turns control of the crime scene back to airport authorities.

- Characterization Phase. The focus is on identifying the BWA through use of reliable detection equipment, performing characterization environmental sampling to determine the location and extent of contamination, and obtaining positive confirmation of the agent using a reliable laboratory. Environmental characteristics of the BWA (such as its survivability on surfaces) as well as potential health consequences to humans and harm to the environment are evaluated to determine what type and degree of decontamination are needed for the affected facility and what public health (medical) measures are needed for persons who were potentially exposed.

- Decontamination Phase. The focus is on preparing and implementing detailed plans for decontamination of contaminated areas. For some nonpersistent biological agents, natural attenuation may be an adequate decontamination option. In cases where contamination is not extensive or the agent is not persistent, application of surface decontaminants or other methods of medical infection control may be effective. For extensive contamination by persistent agents such as Bacillus anthracis, fumigation may be necessary. In those cases, the Decontamination Phase generally begins with source reduction, which involves removing salvageable and nonsalvageable items, and pre-cleaning surfaces to reduce contaminant load. Site preparation includes sealing openings to prevent leaks and 


\section{INTRODUCTION}

setting up equipment. Scenario-specific decontamination reagents and delivery systems are selected, and all systems are pre-tested before carrying out chemical treatments. Decontamination ends when the treatment chemicals have been removed or neutralized and all related decontamination activities, including waste disposal, are complete.

- Clearance Phase. The focus is on determining whether the facility can be reoccupied and airport operations re-established. Appropriate experts review and evaluate key data, such as characterization and clearance sampling results, decontamination process parameters, results from the culture of biological indicators, and quality assurance/quality control (QA/QC) and other relevant information. Remediation goals are examined, and specific criteria are applied to judge the effectiveness of the decontamination process and to determine whether it is safe to reoccupy the facility. Final decisions on clearance are made by local, state, or Federal public health officials and/or government agencies, depending on site-specific jurisdictional authorities and specifics of an incident.

- Restoration Phase. The focus is on preparing the airport for reoccupancy, such as renovating areas that have undergone fumigation.

The focus of this document is on activities associated with Characterization, Decontamination, and Clearance. Appendix A describes considerations for the Notification Phase, and Appendix B describes considerations for the First-Response Phase. These two initial phases, to a large extent, occur before detailed characterization and decontamination activities commence. It is also possible that some of these activities will overlap with and take place concurrently with certain cleanup activities.

This document does not describe public health responses to release of a BWA. If laboratory analytical results confirm the presence of a biological agent, the responsible public health agency involved in the response will commence appropriate public health actions, such as treatment [Centers for Disease Control and Prevention (CDC) 2004] and decontamination of potentially contaminated individuals, distribution of prophylaxis, and medical examinations. See the CDC web site for more information on public health response (http://www.bt.cdc.gov/).

Effective communication with the media and the public is an essential component of an airport remediation and recovery effort. Although the topic is beyond the scope of this document, many helpful resources are available. See, for example, http:/www.epa.gov/NHSRC/pubs/reportWHOhandbook120706.pdf and http://www.bt.cdc.gov/firsthours/. 


\section{INTRODUCTION}

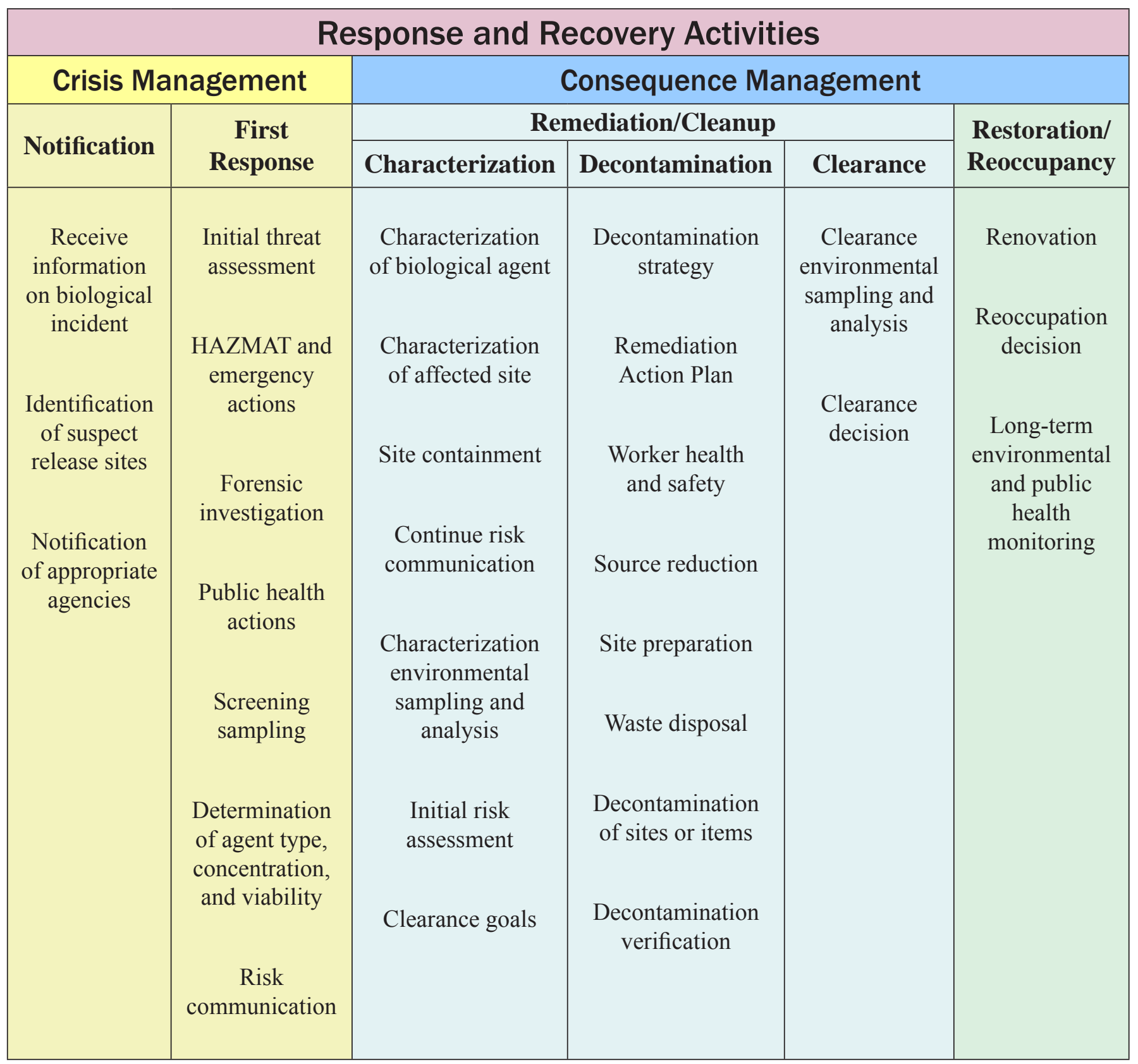

Figure 1-1. Response phases to a biological event. The content of this illustration was developed with interagency cooperation. The focus of Remediation Guidance for Major Airports after a Bioterrorist Attack is on characterization, decontamination, and clearance (areas shaded in blue). 


\section{INTRODUCTION}

\subsection{Basic Scenarios}

Responding to an intentional release of a BWA differs in important ways from responding to a release of a chemical warfare agent (CWA) or hazardous material. In the event of a release of a CWA or a traditional hazardous material, immediate actions must be taken because of the acute toxicity of such agents. Passengers and airport personnel are immediately at risk and may begin to show symptoms within seconds of exposure. Quick action must be taken to contain and stabilize the situation. In contrast, following the release of a BWA, excluding a biotoxin, the hazard may not be immediately apparent. If a release is covert, it may be days or weeks before passengers begin to show symptoms, and during this time, contamination can continue to spread throughout an airport. If a release is made overtly (for example, the release of something suspicious is observed, or some warning information is received), it may not be immediately obvious what must be done to contain the contamination. Depending on the type of organism and the quantity released, different decontamination measures may be required.

A wide variety of potential contamination scenarios are possible. Figure 1-2 shows that potential scenarios range from a single location in which a release is overt, to multiple locations in which releases are covert and only discovered through forensic epidemiology as former passengers begin to show symptoms many days after leaving an airport. This document focuses on contamination by Bacillus anthracis (B. anthracis) spores because they are stable, are the most difficult of known bioterrorism agents to decontaminate, and are much more difficult to inactivate than vegetative cells. B. anthracis spores remain viable for lengthy periods, and they are easily spread by general airflow typical of heated or cooled indoor environments. Because of the focus on B. anthracis spores, this document describes numerous decontamination technologies and reagents, but focuses on sterilants. A sterilant is a substance that destroys all microorganisms on inanimate surfaces, including vegetative and spore forms of bacteria and fungi, as well as viruses.

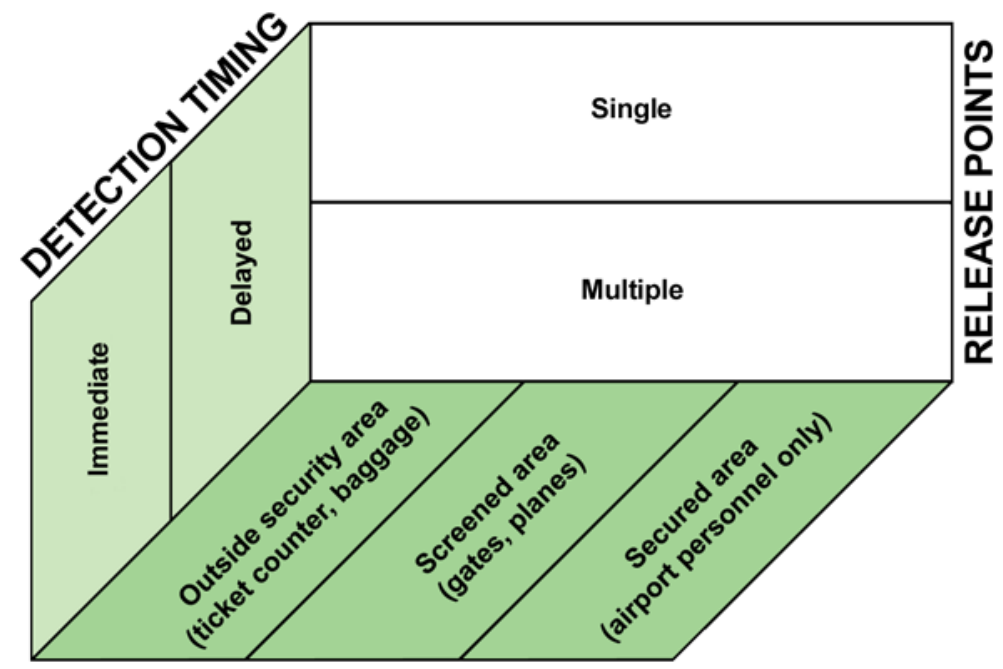

RELEASE SITES

Figure 1-2. Range of potential scenarios for contaminating a major airport following a release of BWAs. 


\section{INTRODUCTION}

As is apparent from Figure 1-2, a sound and site-specific Remediation Action Plan must provide information needed to rapidly characterize and determine decontamination actions for an entire airport (in the case of multiple, covert releases not discovered for days) as well as to isolate and decontaminate a portion of an airport in the case of a small-scale, overt release.

\subsection{Example Airport}

This Remediation Guidance document is general in nature and applies to any large airport. However, an example airport was selected because specific structures can provide concrete examples of concepts. San Francisco International Airport (SFO) was selected as the example airport.

Figure 1-3 shows a schematic of SFO. The airport consists of two domestic terminals (Terminals 1 and 3), a new International Terminal, and Terminal 2 (the former International Terminal now under renovation for use as a domestic terminal). All terminal buildings are connected to form a closed ring. Boarding gates extend outward from the central ring of terminal buildings. Short-term parking for domestic terminals is located in the center of the terminal ring. A rail system with trains to long-term parking, rental car facilities, and the Bay Area Rapid Transit system operates from all terminals. Although contamination will probably spread to such transportation systems and parking areas, this Remediation Guidance document focuses only on the terminal buildings and associated boarding areas.

The terminal buildings and boarding areas at SFO are similar to those at many large airports. In addition to ticket counters, boarding gates, and baggage handling areas, numerous merchants, restaurants, and other vendors are present. The terminals contain museum-quality display cases for artwork, and the International Terminal Building houses a museum. SFO also has baggage screening machines (CTX equipment).

In addition to this document and its appendixes, which are designed to be applicable to most major airports, a data supplement has been developed specifically for SFO. The supplement includes a detailed description of the airport, including sizes, volumes, and materials. It also includes a detailed description of heating, ventilation, and air conditioning (HVAC) systems. To obtain any data supplement referred to in this document, a request must be made directly to the SFO Emergency Planning Operations Division.

\subsection{Cleanup Response Structure}

Figure 1-4 shows the general CONOPS for cleanup of a BWA release at a major airport. This CONOPS conforms to the National Response Framework (NRF) (DHS 2008) and implementation of the National Incident Management System (NIMS 2004). Data Supplement A provides a specific CONOPS, or command structure, for SFO. The CONOPS shows the relationships among organizations involved in the cleanup of a major airport. Figure 1-5 identifies some of the entities that would likely make up the Unified Command, Technical Working Group, and Environmental Clearance Committee. 


\section{INTRODUCTION}

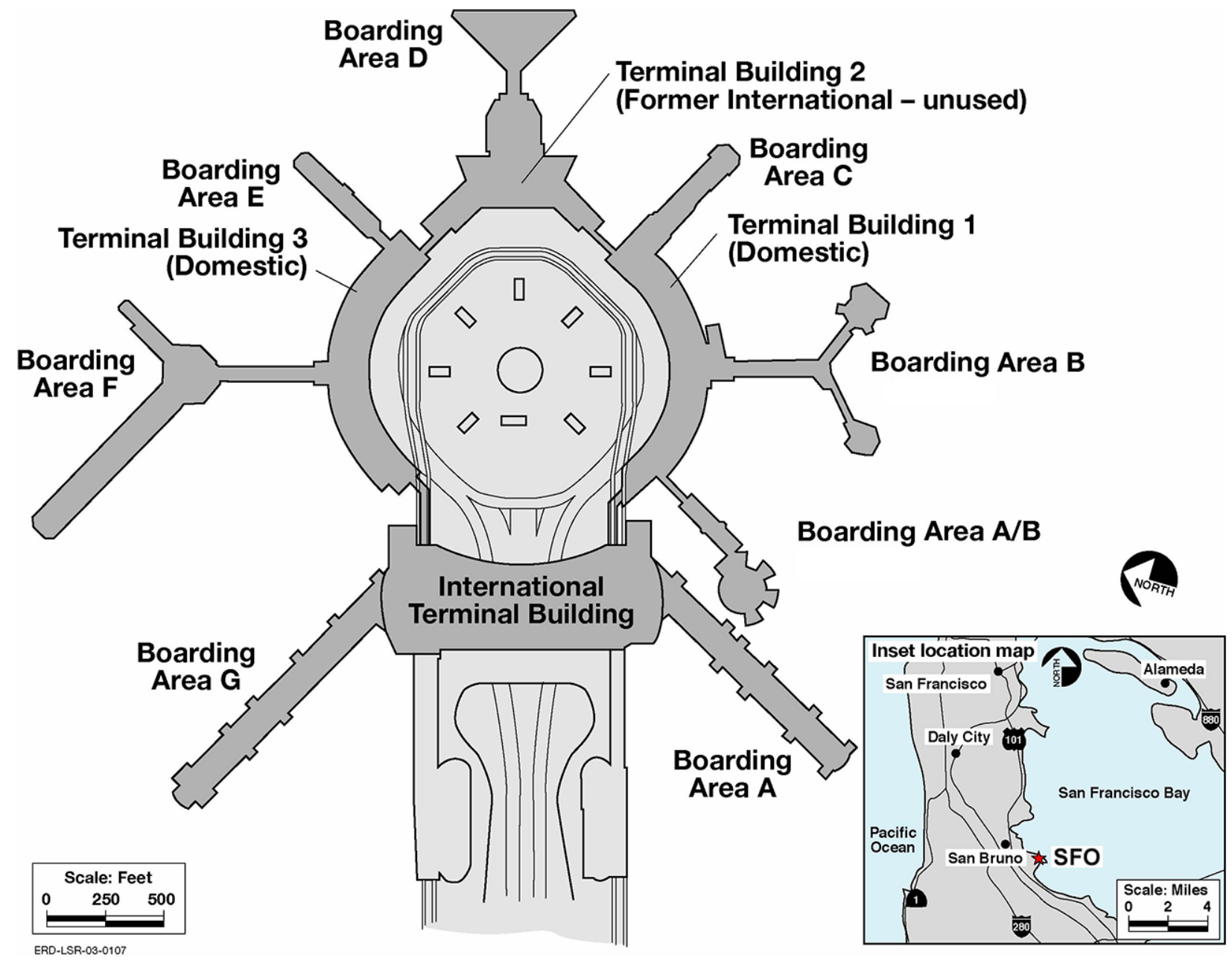

Figure 1-3. Plan view of San Francisco International Airport terminals and boarding areas. 


\section{INTRODUCTION}

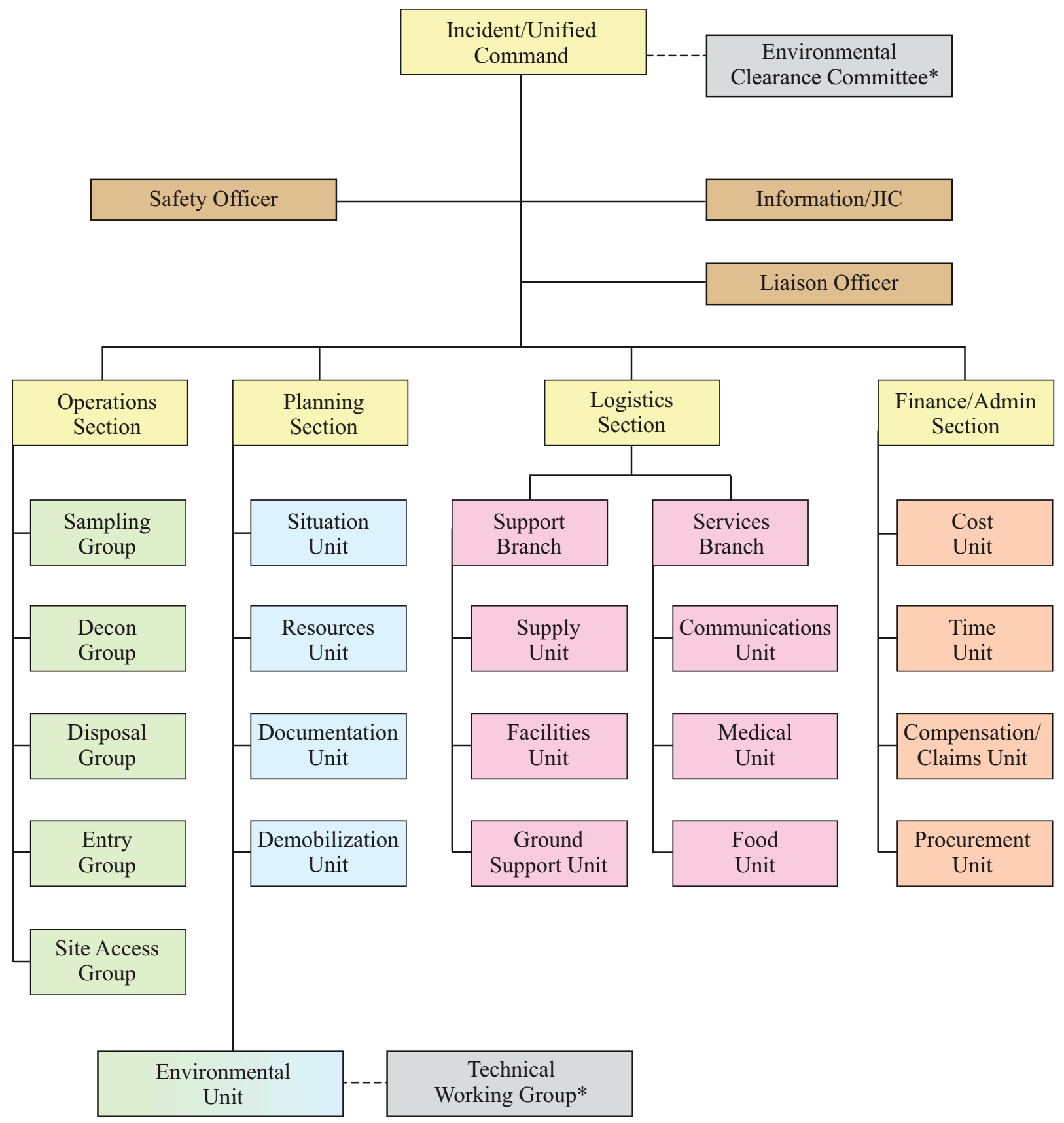

* At the discretion of the UC, the ECC may either report directly to the UC or function under the direction of the Environmental Unit Leader. The TWG may work within the Planning or Operations Section or both. Dashed lines indicate the association of entities not identified in the NRF as required participants within the command structure.

Figure 1-4. Cleanup CONOPS for a B. anthracis release at a major airport. See Figure 1-5 for details. 


\section{INTRODUCTION}

\section{Unified Command}

- Facility representative

- State and local public health

- Emergency management agency

- EPA On-Scene Coordinator

- City representative

\section{Technical Working Group}

Areas of Required Expertise

- Microbiology

- Sterilization science

- Waste disposal (federal and state)

- Ambient air monitoring

- Environmental sampling

- Chemical engineering

- Decontamination methods

Representatives from

- Facility

- $\mathrm{CDC}, \mathrm{NIOSH}$

- EPA

- State and local public health, analytical labs

- Other affected public health agencies

- OSHA

- Private sector

- Universities

\section{Environmental Clearance Committee}

Areas of Required Expertise

- Microbiology

- Epidemiology

- Sterilization science

- Environmental Sampling

- Risk assessment

- Industrial hygiene

Representatives from

- State and local public health department (Chair)

- EPA with ECC experience (Co-chair)

- Local health officer

- CDC

- OSHA

- Stakeholder representatives (ex officio)

Figure 1-5. Components of the Unified Command, Technical Working Group, and Environmental Clearance Committee. 


\section{INTRODUCTION}

The airport's Emergency Operations Center (EOC) may become the location at which on-scene incident command functions are performed, assuming that the EOC remains uncontaminated. In the language of the NRF, this field location for primary, tactical-level functions is referred to as the Incident Command Post (ICP). If the airport's EOC is located within the contamination zone following a biological release, the ICP would relocate to an another facility. Thus, it is important for airport managers to identify alternative facilities that can serve as the EOC or ICP.

An Incident Command (IC) or Unified Command (UC) for cleanup is established at the ICP (which corresponds to the airport's EOC if the EOC is in an uncontaminated location). A UC is formed when more than one agency has incident jurisdiction or when incidents cross political jurisdictions. Agencies work together through designated members of the UC to establish a common set of objectives and strategies and a single Incident Action Plan. The Airport Manager or Airport Emergency Operations Manager would participate in the UC. Other members of the UC could include state and local health emergency management and environmental agencies and the U.S. Environmental Protection Agency (USEPA). In a major incident, a Principal Federal Official may be appointed by the Department of Homeland Security to facilitate Federal support to the overall response. In the language of the NRF, an Area Command is established (1) to oversee the management of multiple incidents that are each being handled by an ICS organization or (2) to oversee the management of large or multiple incidents to which several Incident Management Teams have been assigned. In most cases, the Environmental Unit (EU) within the ICS structure would determine whether to assemble an advisory group of outside technical experts to plan cleanup operations and review all relevant data. Such experts form a Technical Working Group (TWG), and depending on the specific site and incident, this group could provide advice and make recommendations directly to the Incident Commander or Unified Command, or it could operate within the EU of the Incident Command System (ICS), as shown in Figure 1-4. For smaller incidents where a full ICS structure is not established, the TWG could provide advice and recommendations directly to the IC/UC or other organization responsible for cleanup. Given the complex, costly, and timeconsuming nature of fumigations for B. anthracis spores, establishing a TWG is not required but is highly recommended as an effective and efficient way to plan for remediation at sites requiring fumigations. Potential members of the TWG should be identified in advance, meet periodically, and should review this Remediation Guidance document before an incident occurs.

The airport owner, UC, or organization in charge of cleanup also decides whether to assemble an Environmental Clearance Committee (ECC). At most, but not all, sites with fumigations following the 2001 B. anthracis attacks, ECCs consisting of subject matter experts in relevant scientific disciplines were established. If an ECC is established, it functions as an independent body in reviewing relevant characterization environmental sampling data, adherence to key process variables and biological indicator results from fumigation(s), and the nature, placement, and results of clearance environmental sampling. An ECC then makes recommendations on whether facilities are effectively decontaminated. Establishing an ECC is not mandated but is highly recommended as an effective way to make sound clearance decisions and add credibility to the determination that a decontaminated area may be reoccupied. Members of the ECC should be identified in advance and should review this Remediation Guidance document before an incident occurs. It is also highly recommended that the ECC be convened at the initiation of cleanup activities. The makeup and functions of an ECC are discussed in more detail in Section 4.

Although this general CONOPS does not assume that Federal assistance is requested, such a request is likely. If the DHS determines that there is a major incident, a Principle Federal Official (PFO) may be designated and a Joint Field Office (JFO) would be established to coordinate Federal support. The governor of the state in 
which the airport is located may also request that the U.S. President declare an emergency under the Stafford Act. If the request were granted, the Federal Emergency Management Agency (FEMA) would issue mission assignments to appropriate Federal agencies for various functions that support the response. The USEPA would likely receive mission assignments for decontamination and other remediation activities under Emergency Support Function (ESF) \#10 of the NRF.

\subsection{Pre-Incident Planning}

Reducing the time required to restore operations at an airport is a major goal of remediation planning and a consistent theme throughout this document. Airport decision makers can address many important functions well in advance of a biological contamination event. Table 1-1 identifies essential pre-incident planning activities related to the cleanup response structure.

Table 1-1. Summary of concept-of-operations actions to be taken prior to a biological attack.

\begin{tabular}{|c|c|}
\hline Responsible Personnel & Pre-Incident Actions \\
\hline $\begin{array}{l}\text { Airport authority decision } \\
\text { makers }\end{array}$ & $\begin{array}{l}\text { - Identify alternative locations for an Incident Command Post, preferably near } \\
\text { the airport, but offsite in the event that an Emergency Operations Center is } \\
\text { contaminated with a biological agent. } \\
\text { - Identify potential members of a Technical Working Group (TWG). The TWG } \\
\text { should review this Remediation Guidance document. } \\
\text { - Identify potential members of an Environmental Clearance Committee (ECC). } \\
\text { The ECC should review this Remediation Guidance document. } \\
\text { - Conduct periodic training exercises with likely command personnel, including } \\
\text { TWG members, and other responder and agency representatives. Ensure they } \\
\text { have appropriate health and safety training. }\end{array}$ \\
\hline
\end{tabular}

\subsection{Section 1 References}

Centers for Disease Control and Prevention (CDC) (September 2004), Fact Sheet: Isolation and Quarantine; Fact Sheet: Legal Authorities for Isolation and Quarantine; and Questions and Answers: Legal Authorities for Isolation and Quarantine, Department of Health and Human Services, Centers for Disease Control and Prevention.

DHS (March 2008), Department of Homeland Security, National Response Framework; available at http://www.fema.gov/emergency/nrf/.

Department of Transportation (DOT) (2006 draft), National Aviation Resource Manual for Communicable Diseases That Are Quarantinable; This resource has information on quarantine, role of the Officer in Charge of Quarantine Stations, and role of the Quarantine Medical Officer.

NIMS (March 1, 2004), National Incident Management System; document available from FEMA at 1-800-480-2520, Option 4, ask for FEMA 501.

Raber, E., J. M. Hirabayashi, S. P. Mancieri, A. L. Jin, K. J. Folks, T. M. Carlsen, and P. Estacio (2002), "Chemical and Biological Agent Incident Response and Decision Process for Civilian and Public Sector Facilities," Risk Analysis 22(2), 195-202. 


\section{CHARACTERIZATION}

2

\section{Characterization}

Cleanup activities begin with the Characterization Phase, which consists of assessing the extent of contamination and gathering information needed to develop a plan for remediation. The cleanup command structure, described in Section 1.4, is likely to be in place before completing first-response activities. If a $\mathrm{UC}$ for cleanup is not yet established, it is activated now. The UC or organization in charge mobilizes the resources required for characterization and begins to activate resources needed for decontamination and clearance. If possible, such resources should be assembled before first-response activities are complete. Any data that were collected during first-response are compiled by the Planning Section's Situation Unit and assessed. For instance, initial environmental sampling (also sometimes called screening sampling), which will have been done earlier by law enforcement agencies (assumed to be the FBI) or HAZMAT teams, is used to approximate the locations of contamination. Those teams will have established an exclusion or "hot" (contaminated) zone, a contaminant-reduction or "warm" zone (transition area where response personnel are decontaminated), and a support or "cold" zone, where contamination is unlikely. From the results of screening sampling, the zones may need to be adjusted. Some decontamination may be necessary to ensure that hot and cold areas are, in fact, separated.

Positive confirmation of the BWA is obtained from a reliable laboratory. Analysis by a Laboratory Response Network (LRN) laboratory is recommended to ensure that standardized methods are employed. Characterization data are collected to confirm the extent of contamination so that a determination can be made as to what decontamination activities are needed, and to make initial decisions about the necessary clearance samples. A summary of actions taken during characterization and the personnel responsible for those actions is provided at the end of Section 2.

The recommended approach to environmental sampling is simple: Work through an airport systematically and thoroughly, area by area. In each area:

- Assess existing information, including the likelihood of contamination.

- Identify any questions to be answered, hypotheses to be tested, and decisions that need to be made.

- Decide how to sample to answer questions, test hypotheses, or support decisions.

- Combine assessments and selections of sampling zones and units into a written sampling plan.

- Obtain necessary approvals, and execute the characterization plan.

- Assess the sampling results and perform more sampling if necessary, or move to the next phase of cleanup.

\subsection{Initiate Startup Activities and Mobilize Resources}

Characterization activities can begin as soon as contamination by a BWA is suspected, or immediately upon confirmation of such contamination even if First-Response Phase activities are not complete. The UC or organization in charge establishes an ICS structure, which may include a TWG and ECC, and mobilizes other resources (personnel and equipment) needed for characterization. Resource personnel who should be ready to respond on short notice include: 


\section{CHARACTERIZATION}

- Sampling teams, which should have up-to-date training.

- Analytical laboratories, which should be part of the LRN or approved by the CDC. If contamination is suspected or confirmed to be widespread, thereby requiring many characterization environmental samples, the primary laboratory may have insufficient capacity, and additional laboratories would be recruited.

- Airport personnel who maintain current information on the physical aspects of a facility, such as architectural drawings and operation of the heating, ventilation, and air conditioning (HVAC) systems.

- Dispersion-modeling experts.

- Data management and documentation specialists to organize a database(s) and establish a QA/QC plan.

- Contractors able to construct containment and isolation barriers under hazardous conditions.

- Contractors with expertise in waste management, owners of waste-disposal facilities, and wastewater management authorities.

If an ECC has been formed, they should be available for consultation. From descriptions of how ECCs functioned at $B$. anthracis contaminated sites in 2001, the ECC need not have a major role during characterization. To maintain independence, it is best if ECC members are not members of any team planning or performing characterization work; however, the ECC should be kept informed of the plans and work.

The startup time for characterization will be reduced if resources are identified in advance. To assist with advance preparation, Appendix $\mathrm{C}$ lists some methods for environmental sampling and analysis for B. anthracis, and Appendix F lists some available resources for remediation. Most USEPA regional offices maintain a list of qualified environmental remediation contractors. USEPA On Scene Coordinators have access to contractors trained to perform environmental sampling. The USEPA National Decontamination Team is a valuable source of information. Airport planners should be familiar with resources available through their local USEPA offices and should establish a working relationship with personnel at those offices before any incident. The airport should have pre-existing contacts with a primary LRN laboratory and should know in advance the laboratory's capacity under normal and surge circumstances. Airport planners should establish contact with other LRN laboratories that can analyze additional samples.

Data management systems must be in place before any characterization occurs. It is imperative to have a data-collection, processing, storage, and reporting system in place that identifies specific data-quality objectives, ensures data integrity, and can be managed effectively. This is especially important if multiple sampling teams from more than one outside organization (contractors) collect the samples. The value of sampling is undermined if the sampling itself is not well documented. All samples should be labeled, and the specific location of each sample should be recorded so that analytical results can be used to direct decontamination activities, update conceptual models, and revise modeling output. Electronic database management tools, which combine electronic facility drawings, bar code tracking, and data visualization are under development and should be available for managing future characterization data. The Characterization Sampling and Analysis Plan (SAP), discussed later in this section, should describe how data management is conducted. See Appendix I for a template to facilitate preparing a Characterization SAP. Digital photographs of every sampling location can help document the sampling activities. 
In summary, airport decision makers should identify the characterization resources shown in Table 2-1 in advance of an incident so that such resources can be mobilized immediately. Phone numbers and contacts should be verified at least once a year. Appendix L is a summary of contacts for all phases of cleanup. Figure 2-1 summarizes the pre-incident, startup, and information-gathering activities associated with the Characterization Phase.

Table 2-1. Site characterization resources that should be identified in advance. (Airport authority to fill in information.)

\begin{tabular}{|l|l|l|}
\hline \multicolumn{1}{|c|}{ Resource } & Contact & Phone \\
\hline Members of Unified Command or organization in charge & & \\
\hline Members of Technical Working Group & & \\
\hline Members of Environmental Clearance Committee & & \\
\hline Primary LRN analytical laboratory & & \\
\hline Secondary LRN analytical laboratory & & \\
\hline Sampling team(s) and contractor(s) & & \\
\hline Data management and documentation specialists & & \\
\hline Agent air monitoring team and contractor & & \\
\hline Personal protective equipment (PPE) rental & & \\
\hline Facility engineering and construction team(s) & & \\
\hline Air-transport modeling team and contractor & & \\
\hline Centers for Disease Control and Prevention (CDC) & & \\
\hline U.S. Environmental Protection Agency (USEPA) & & \\
\hline Waste-disposal resource personnel & & \\
\hline Wastewater management authorities & & \\
\hline
\end{tabular}

\subsection{Perform Initial Assessment}

Initial assessment of an environmental hazard (sometimes referred to as a conceptual site model) typically includes identification of the (1) potential sources of contaminants, (2) known or expected locations of contaminants, (3) media that are contaminated or may become contaminated, and (4) exposure scenariosthat is, locations of human or ecological receptors. Errors made during the initial assessment may be perpetuated throughout all subsequent steps of cleanup. Conversely, an accurate initial assessment guides remaining actions down an appropriate path. Information about (1) potential sources of contaminants should be available from the First-Response Phase, which includes forensic investigation. The type of information depends on the method of release (see Sections 1.2 and 2.2.2), such as overt or covert, aerosol or explosive, and so forth. The assessment of (2) known or expected locations of contaminants is discussed in Section 2.2.2 as well as subsequently in Section 2.2.10 in the context of conceptual and mathematical modeling. A major airport is a complex facility that will likely be remediated in manageable pieces. This topic is discussed in Section 2.2.6 and Appendixes D and H. Some airport areas, especially those that are separate or distant from the location of an overt release, may be assessed separately. Some information about (3) media that are, or can become, contaminated should also be inherited from the First-Response Phase. This topic is discussed in Section 2.2.7 and Section 2.2.8. The topic of (4) exposure scenarios is discussed in Section 2.2.9. 


\section{CHARACTERIZATION}
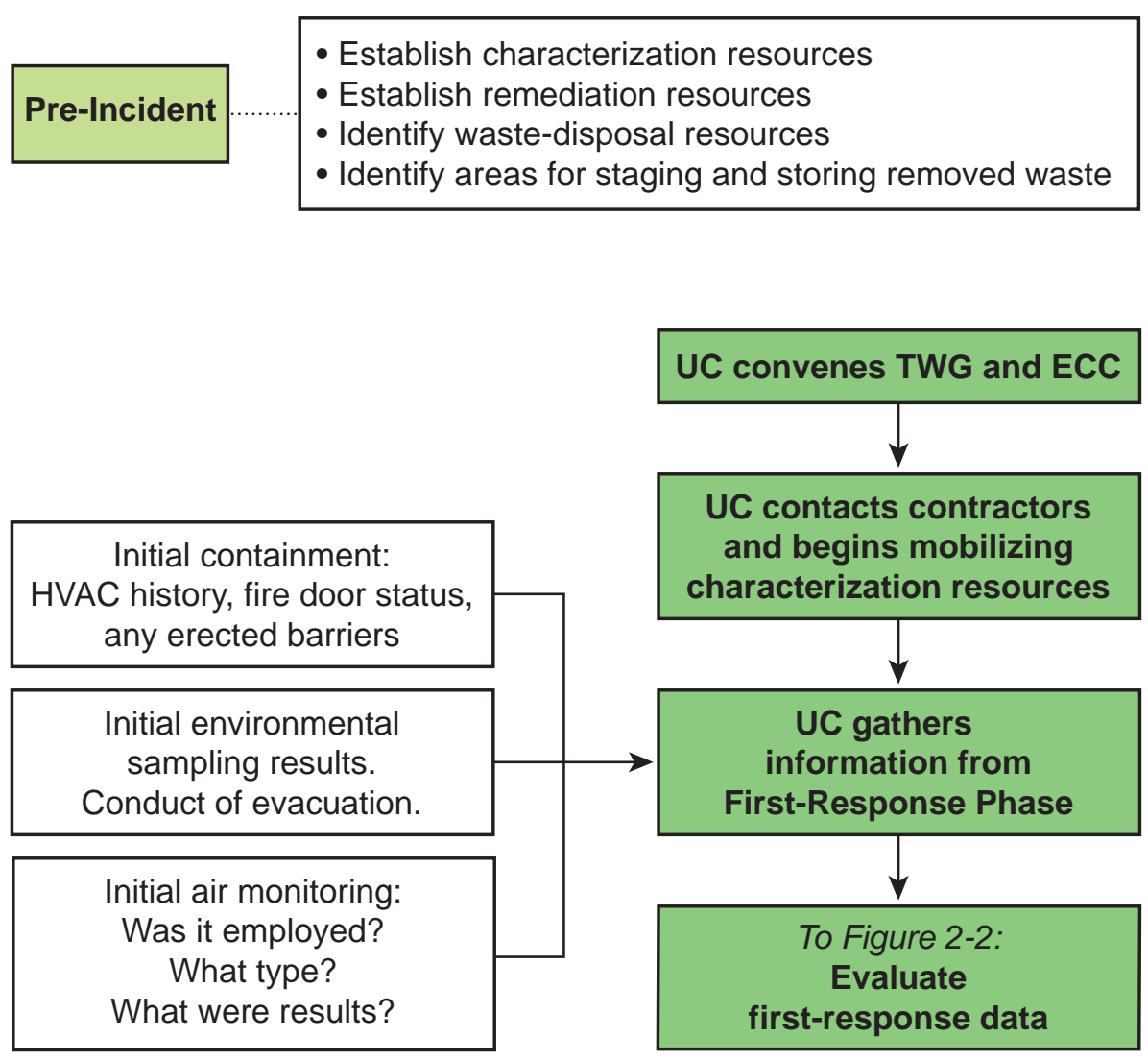

Figure 2-1. Pre-incident actions, startup activities, and information gathering during first response.

Initial environmental sampling data and any other data collected during first response should be compiled by the Planning Section's Situation Unit and turned over to the EU for use in planning subsequent cleanup. The FBI has indicated a willingness to share initial environmental sampling data, as appropriate, with the UC. If the data were not made available, airport cleanup will be delayed. If compatible with evidence-collection procedures, the FBI will allow some cleanup activities, such as characterization environmental sampling, to begin while it completes the collection of criminal and forensic evidence.

\subsubsection{Create New Health and Safety Plan}

A Health and Safety Plan (HASP) is required by the Occupational Safety and Health Administration (OSHA) (29 CFR 1910.120) for characterization, decontamination, and clearance activities. The HASP describes physical, chemical, and biological hazards at the site and should include procedures for discovering any unknown hazard. The plan describes the establishment of HAZMAT hot (contaminated), cold (uncontaminated), and warm (intermediate) zones for use by hot-zone entry personnel; personal protective equipment (PPE) requirements; personal decontamination procedures; and emergency procedures to be used by sampling and decontamination personnel. If an airport has pre-designated staging areas, a substantial portion of the HASP for characterization can be written prior to an incident. A model HASP for 


\section{CHARACTERIZATION}

B. anthracis response is provided by OSHA at their web site (current as of January 2006), as follows:

- http://www.osha.gov/dep/anthrax/hasp

Additional helpful resources include:

Anthrax eTool (OSHA): http://www.osha.gov/SLTC/etools/anthrax/index.html

Technical Assistance for Anthrax Response (November 2003), Chapter 5: Health and Safety

Considerations (NRT): www.nrt.org

The model HASP could be used as a basis for creating an airport-specific HASP. Ultimately the UC, through the Site Safety Officer, is responsible for ensuring the health and safety of all responding entities. The Incident Command System (ICS) planning process includes HASP development activities to ensure that consistent and coordinated health and safety measures are in place for all responding entities. The Site Safety Officer and Logistics Section's Medical Unit create the HASP. These are the same personnel who vaccinate or provide antibiotics to appropriate cleanup and response personnel.

\subsubsection{Evaluate Initial Release and First-Response Data}

The key question the UC must address at the beginning of the Characterization Phase is: what parts of the airport are definitely contaminated, probably contaminated, or not likely contaminated? Decision makers need to make judgments about how much confidence can be placed in answers based on information collected during first response. The process leads to identifying what data must be obtained during characterization (i.e., identifying the data gaps). Appendixes $\mathrm{H}$ and I are tools to help with the process. The CDC may mobilize its response assets to assist local responders in evaluating health risk and contamination pathways. Expertise may be drawn from the National Institute for Occupational Safety and Health (NIOSH) and the Agency for Toxic Substances and Disease Registry (ATSDR) to conduct preliminary characterization activities.

Following the incident, first responders will have collected initial environmental samples, and a local public health laboratory that is a member of the LRN will have tested for the presence of a biological agent. Initial environmental samples are likely to be surface samples. Confirmation may depend on the specific organism involved, especially in the case of a covert release. Some BWAs do not survive long on environmental surfaces.

It is essential that samples be collected and analyzed using methods effective for detecting biological agents. Use of a method based on the polymerase chain reaction (PCR) followed by laboratory confirmation through sample culture would confirm that a release of $B$. anthracis spores occurred. Faster methods for determining the viability of a BWA using combined PCR and culture are under development and may be available for future characterization actions. After the presence of a biological agent has been confirmed in at least one sample, the startup activities described in Section 2.1 begin. Figure 2-2 summarizes the initial assessment of first response data and subsequent steps leading to characterization. 


\section{CHARACTERIZATION}

\subsubsection{Evaluate Other Sources of Data}

Security camera recordings and eyewitness accounts might provide some information about the extent of contamination. Data on ambient conditions (temperature and humidity, HVAC mode of operation, outside winds, and time of day) are important for characterization. Data on ambient conditions can be used to model the spread of agent and to help estimate the exposure of potentially affected individuals. If the BioWatch program (Fitch et al. 2002; also see http://www.dhs.gov/index) operates any samplers near the airport, such data should be obtained and reviewed.

Information about the movement of people or electric carts after a release should be sought. For example, contamination may have been initially disseminated from a point or line source, then spread by foot or vehicular traffic, or it may have been intentionally introduced into the HVAC system. Facility personnel are responsible for providing blueprints of HVAC systems and areas of operation to the Planning Section's Documentation Unit. Different methods of dispersal would result in different patterns of contamination, and sampling should discover the resultant pattern. Such input helps the EU provide information to the UC to decide what parts of the airport require decontamination.

\subsubsection{Evaluate Immediate Data Needs}

After reviewing available initial environmental sampling data, the EU may identify a need for information that can be filled immediately and before performing a complete and properly designed characterization sampling effort. Such data should be collected; however, the process is not a substitute for full characterization.

\subsubsection{Consider Immediate Source Reduction}

As the EU and the Operations Section plan for the main decontamination actions (e.g., fumigation in the case of aerosolized B. anthracis), it may become apparent that there are localized areas with especially high levels of contamination, particularly in the vicinity of the release. If so, immediate, localized decontamination should be considered. If dense deposits of B. anthracis are present on the floor surrounding the release device, they could be removed by a surface application of diluted, buffered bleach solution (see Section 3.5.2.1) before fumigation. Because refined B. anthracis spores are easily re-aerosolized and spread by activities in the vicinity, prompt source reduction could prevent spreading of the contamination. 


\section{CHARACTERIZATION}

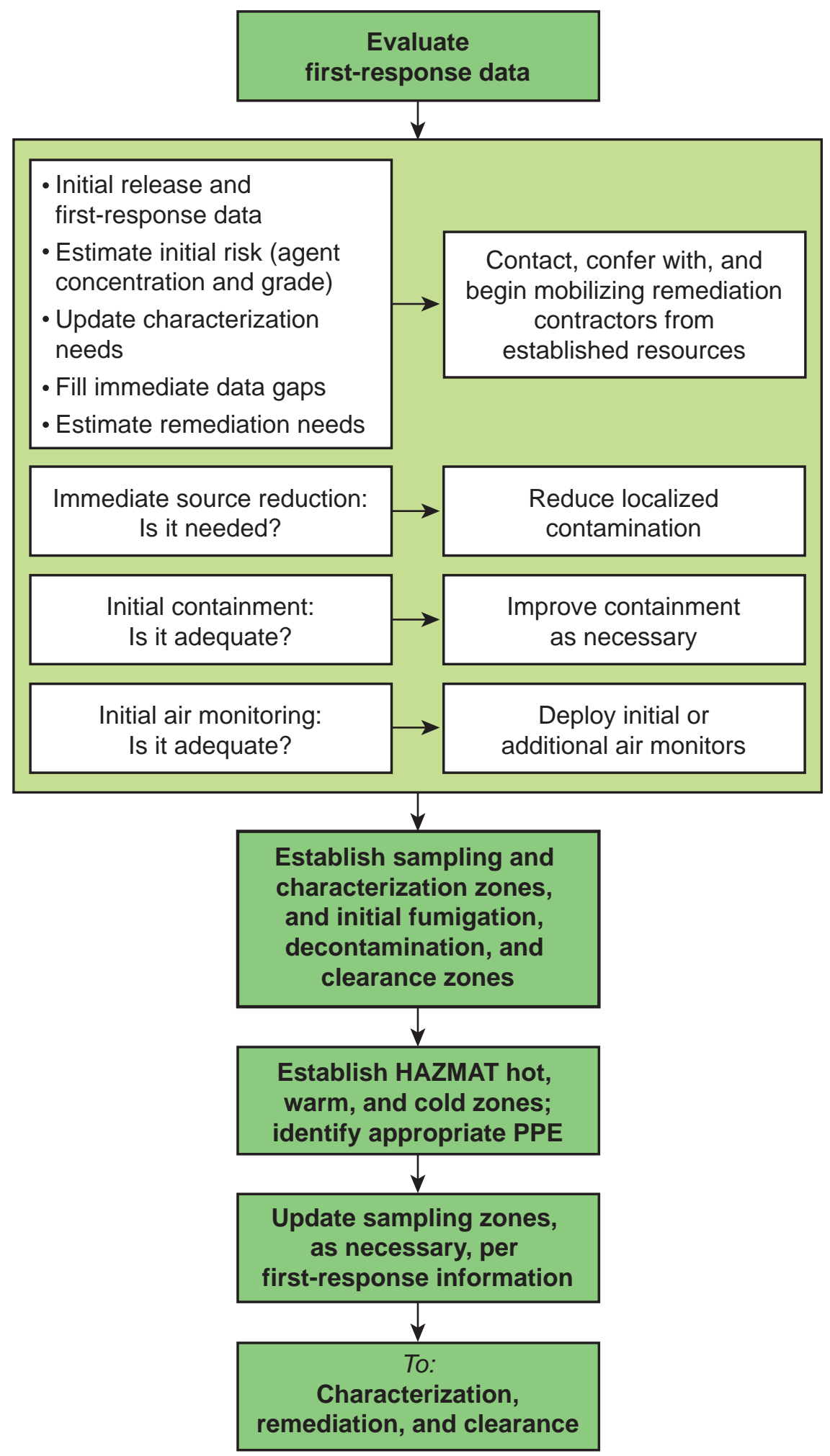

Figure 2-2. Evaluation of first-response data and steps leading to characterization. 


\section{CHARACTERIZATION}

Depending on the method of decontamination, it may be helpful or necessary to remove various items before decontamination begins. Items such as food supplies will probably be unfit or unacceptable for further use no matter how effective the subsequent decontamination. Such items should be removed at the outset. Section 3.5.1 discusses source reduction in more detail.

\subsubsection{Organize Airport into Zones}

It will probably be appropriate and expedient to assess the airport area-by-area. For example, if an overt B. anthracis release occurs in a specific boarding area of the airport, it may seem unlikely that the agent reached the far end of other boarding areas. If these rough, initial assessments are supported by sampling results, then subsequent management of BAG and BAB will be different. Thus, at the outset of cleanup and at the largest scale, it makes sense at SFO to consider the International Terminal (IT) with its two boarding areas as one large section of the airport, Terminal 1 with its boarding areas as another, and so on. Such an approach is consistent with USEPA guidance regarding environmental remediation, namely, "If the problem is complex, the team may consider breaking it into more manageable pieces, which might be addressed by separate studies. Priorities may be assigned to individual segments of the problem and the relationship between the segments examined." (USEPA 2000.)

The Multi-Agency Radiation Survey and Site Investigation Manual (MARSSIM) is radiation-specific; however it is a useful reference for sample planning. The MARSSIM is referenced in Appendix E.7 of this document.

This Remediation Guidance document uses the following terminology to refer to separate areas of the airport during the cleanup process:

- Any discrete section of, or area within, the airport that is viewed as a unit for the purpose of sampling can be called a sampling zone. This term is used generally and without reference to a particular phase.

- As work progresses during characterization, it will be helpful to speak of "characterizing the first floor," "characterizing the second floor," "characterizing the boarding area," "characterizing the departure lobby," and so on. The term characterization zone refers to this level of organization.

- If fumigation is needed, it will be necessary to isolate each area requiring fumigation. Such areas are fumigation zones.

- If any areas need decontamination, but not fumigation, it may be useful to proceed with the decontamination area-by-area rather than simultaneously. Such areas are surface decontamination zones.

- After decontamination, any discrete section or area in the airport that is viewed as a unit for the purpose of making the clearance decision can be called a clearance zone. Where fumigation is used, a fumigation zone naturally becomes a clearance zone. 


\section{CHARACTERIZATION}

As part of the preparation for a potential BWA incident, airport planners should assess the facility layout and identify potential sampling, characterization, fumigation, or decontamination zones. Preparation in advance - when access is not complicated by the presence of contamination-will be much easier than after a BWA attack has occurred. Identifying zones in advance will also help in coding and managing the massive amounts of data that will be collected during an actual event. Consultation with a fumigation contractor to help determine appropriate potential fumigation zones is an essential part of the process. Further discussion and examples are in Appendix D. Appendix H contains templates to help with the process.

Figure 2-3 shows four potential surface decontamination zones at SFO, and some potential characterization zones in the terminal buildings. A detailed list of potential sampling zones for the SFO International Terminal is also available in Data Supplement D. At the time that airport management identifies potential zones, it should also identify areas at the airport that can be used, or cleared, for staging and storing waste materials that will be removed during decontamination activities. 


\section{CHARACTERIZATION}

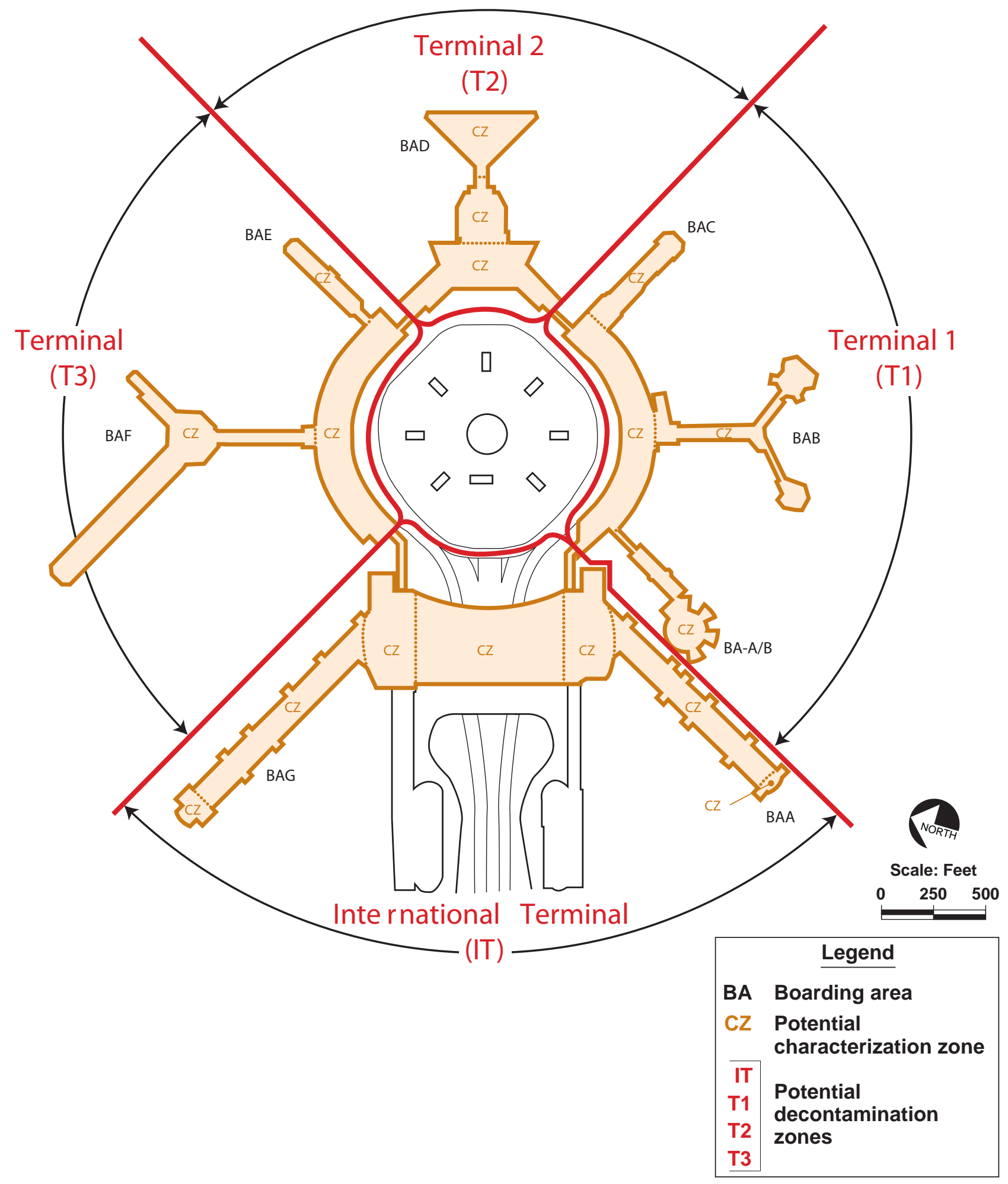

Figure 2-3. Potential decontamination zones and characterization zones at SFO. 


\section{CHARACTERIZATION}

\subsubsection{Review Existing Containment and Isolation}

It is important that the spread of BWA contamination be limited as much as possible. Measures to prevent such spread are referred to as containment. Isolation refers to measures taken to prevent the release and spread of fumigant into unwanted areas. Early during the cleanup process, the EU should review any containment measures that were used during the First-Response Phase and decide whether additional containment is necessary. Airports should identify in advance some potential locations for rapid construction of barriers. Potential barrier locations include connector halls between major terminal areas and fire doors. In addition to their potential for containment during first response and at the beginning of cleanup, such locations might be used later for isolation purposes to form decontamination zones or fumigation zones.

To ensure complete isolation (that is, to prevent the release of fumigant), it is necessary to use either sprayable foam or caulking to seal doors and cracks or to install barriers consisting of plastic polyethylene sheets covered with plywood to prevent puncture. Doors, windows, and external HVAC registers may also need to be caulked and sealed.

Negative air units (NAUs, also known as negative air machines, or NAMs) can be deployed in areas of known or suspected contamination. NAUs subject the area to a slight negative pressure to ensure that an agent remains in the contaminated area (see also Section 3.4 and Appendix F). However, the need to procure or fabricate NAUs, ducts, piping, and other major components may limit the ability to field NAUs during the early stages of characterization. The potential for NAUs to affect the extent of contamination must be assessed. If there is a possibility that the units will move or redistribute particles within a zone, especially if the movement takes place after characterization sampling, NAUs should not be used. Use of NAUs has been a topic of debate in previous remediations; decisions should be made on a case-by-case basis.

\subsubsection{Evaluate Potential Release to the Outside}

An immediate assessment must be made by the EU of the potential for a release of contamination outside the airport buildings. Because B. anthracis spores can spread quickly through the movement of people and reaerosolization (Weis et al. 2002), escape to the outside is a serious possibility. Potential escape paths include HVAC exhausts, storm drains, sanitary sewers, and tracking by people moving out of a contaminated area. Evaluation can start with a method to estimate the amount of agent potentially released to the outside environment and to determine the likely fate of the agent in the environment. Consider placing air samplers outside the airport and in locations near potential escape pathways (see Section 2.2.11, Assess the Need for Air Monitoring).

Aircraft, airport vehicles, rental cars, subways, trains, and the like may have to be grounded and secured because of the potential for cross-contamination. Such actions will probably take place as soon as a BWA event has been confirmed. If not, they should be done at this time.

Because many putative BWAs are zoonotic pathogens, potential spread into the surrounding population of susceptible animals must also be considered. If the pathogen is vector borne, vector-control measures may need to be considered.

\subsubsection{Assess Availability of Agent to Cause Injury or Disease}

Any B. anthracis poses a threat. However, if the results of an assessment show that there is no potential for injury or disease (e.g., the release is totally contained and eliminated during first-response activities), then no further action would be necessary. 


\section{CHARACTERIZATION}

The EU should make a general assessment of exposure potential starting with results from emergency response and forensic environmental sampling data. The EU should work closely with FBI scientists or others and obtain any available data on agent characteristics, amount of agent, mode of release, and initial sampling results. This information together with results from any modeling (Section 2.2.10) is used to determine the immediate and future availability of spores to cause harm.

Air monitoring can help determine whether members of the public were exposed prior to cessation of operations. If a covert release were suspected, air monitoring would measure agent air concentrations that the public was exposed to at the time air monitoring began. Because the release location would probably be known imprecisely at best, air monitors for this purpose should be placed throughout the airport. See also Section 2.2.11.

\subsubsection{Perform Modeling}

It is important to assess the possible extent of contamination within an airport beyond what has been confirmed during the First-Response Phase. Conceptual or physical modeling of agent dispersal is used for this purpose, and it drives hypotheses that will be tested in the characterization sampling strategies and plans. Information on airflow within the affected area at the suspected time of release must be obtained to evaluate the potential spread of agent. For B. anthracis, the method used to formulate the spore product greatly influences aerodynamic properties and spread. Operation of HVAC systems and the air balance within an airport have a major influence on the spread of B. anthracis spores or other BWAs. Such information should be available from airport maintenance and engineering staff. Data Supplements B and C contain such data for SFO. Weather conditions, such as wind speed and direction outside the airport, should also be considered. B. anthracis spores can spread quickly through the movement of people, re-aerosolization, and cross-contamination of objects (Weis et al. 2002). The method of release, time since release, and quantity released are necessary to estimate the spread of spores. The quantity released probably will not be known and will need to be estimated.

One approach to modeling is to develop a conceptual model for the spread of agent using information such as the HVAC mode of operation at the time of release and potential transport of agent via foot traffic. Spread via foot traffic might be estimated from interviews with people who were initially present and from surveillance cameras. A second approach is computer-assisted mathematical and physical modeling of agent dispersion. Mathematical modeling may or may not be undertaken, depending on available resources, whether the necessary input information is available, and whether or not the UC decides such modeling is worthwhile. Expertise in mathematical modeling is available from the national laboratories, among other places. Developing the necessary input information for mathematical and physical modeling must be done in advance. Conceptual or mathematical models can help guide the selection of locations, types, and numbers of samples to be collected.

\subsubsection{Assess the Need for Air Monitoring}

After an overt release, agent air monitoring may have been initiated during first response. After a covert release, neither the time nor location of the release is known, and surface deposition may be light. Air sampling can help confirm the presence and extent of such a release. 


\section{CHARACTERIZATION}

Agent air monitoring should be conducted in staging areas adjacent to HAZMAT hot zones to detect any spread from those areas arising from activities such as entering and exiting. Ongoing air monitoring during the cleanup phase can provide additional information concerning the hazard, potential exposure of workers to the bioagent, and effectiveness of cleanup activities. Agent air monitoring, if any, should be conducted using standard protocols (see Appendix C). Figure 2-4 summarizes considerations relevant to air modeling and air monitoring.

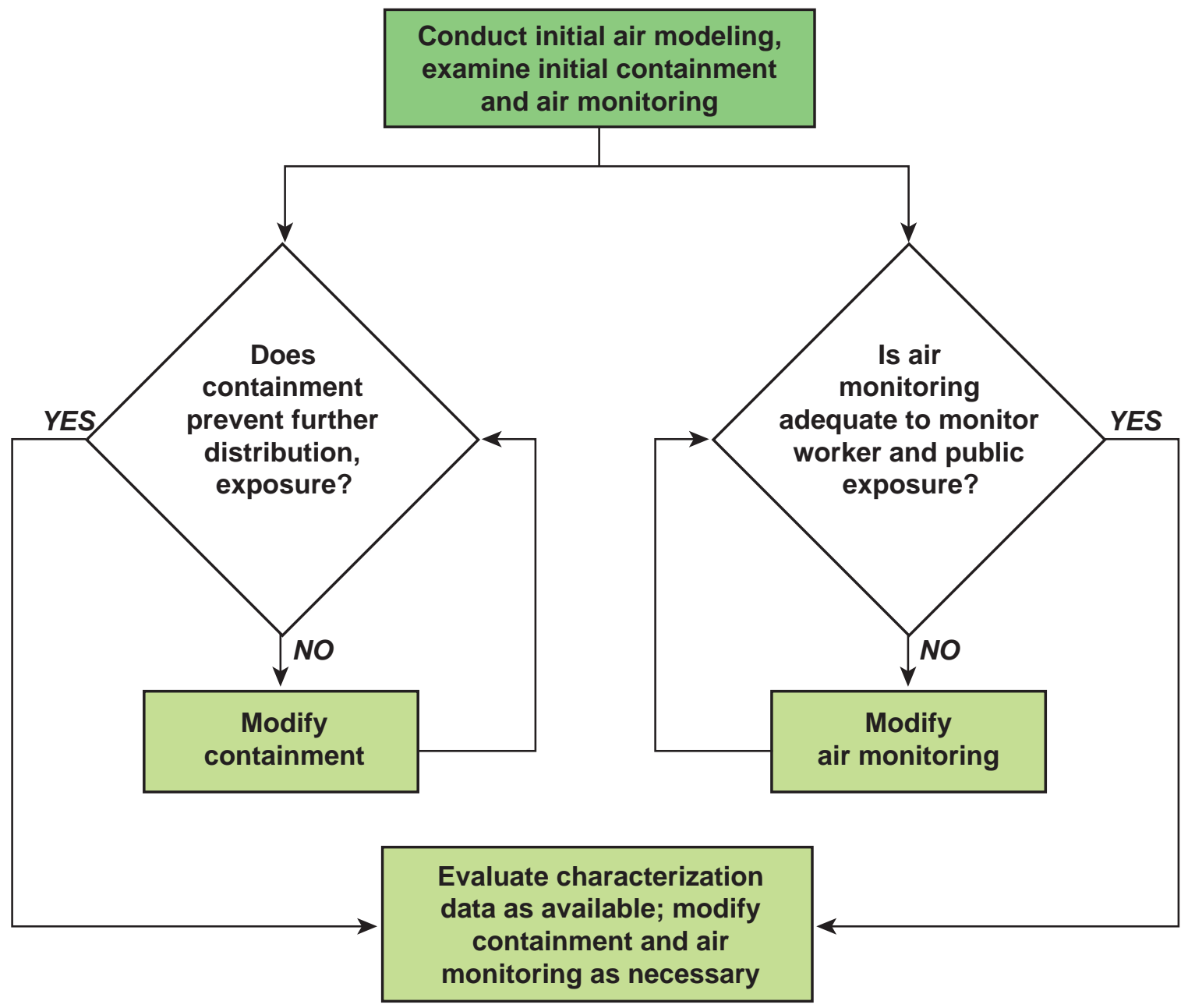

Figure 2-4. Evaluation of air modeling, air monitoring, and agent containment. 


\section{CHARACTERIZATION}

\subsection{Plan for Characterization Environmental Sampling}

The goals of characterization are to determine the magnitude and extent of cleanup required, as well as the source of contamination, where and how it might have spread, and who might have been or could be exposed to contamination. Characterization provides the information needed to design the cleanup effort. All characterization sampling should be designed to answer specific questions identified before sampling begins. Initial screening sampling, conducted during First-Response activities, provides preliminary hypotheses about the extent of contamination. During characterization, the hypotheses are tested, further hypotheses are developed and tested, and a more complete assessment of the condition of the facility is developed. Sampling that is done for this purpose is documented in a Characterization Sampling and Analysis Plan (SAP) that is developed and written by the EU or the Sampling Group within the Operations Section. It describes the sampling strategies that are selected, specifies where to sample, and includes a variety of supporting information. Appendix I is a template to assist in developing the Characterization SAP.

Characterization must be thorough, well documented, and provide sufficient data to support decisions. The entire cleanup process can be viewed as a series of decisions, such as whether or not various parts of the facility need decontamination, how to decontaminate those parts that do, and whether or not to return the facility to service. The templates in Appendixes $\mathrm{H}$ and I outline a process for systematically assessing the airport zone-by-zone, identifying the decisions that need to be made in each area and the information necessary to support those decisions, and deciding how to gather the information through sampling.

Figure 2-5 shows the principal activities that take place throughout the Characterization Phase. Refer to this illustration in the following discussion.

\subsubsection{Develop Characterization Strategies}

Characterization environmental sampling is often described as being done to assess the nature and extent of contamination. The phrase, "extent of contamination" suggests a type of sampling in which the approximate boundaries of contamination are identified - for example, at what distance from a release location the level of contamination is nondetectable. It is necessary to decide how precisely the extent of contamination needs to be determined. For example, is it necessary to determine such a boundary to within 5 feet, 10 feet, or 100 feet? At a minimum, the search for extent could be just sufficient to decide whether or not decontamination is needed. More comprehensively, the search for extent could be done to compare characterization samples with future clearance samples, or to learn about how the agent was dispersed. If rapid cleanup is a high priority, or if resources are limited as might be the case if multiple airports are attacked simultaneously, it will be helpful to more narrowly focus the characterization sampling. For more focused characterization sampling, it is useful to separately identify the following three purposes of characterization sampling:

1. To understand the fate and transport of the contaminant.

2. To gather information needed to design the decontamination approach.

3. To gather information for future comparison with clearance sampling results. 


\section{CHARACTERIZATION}

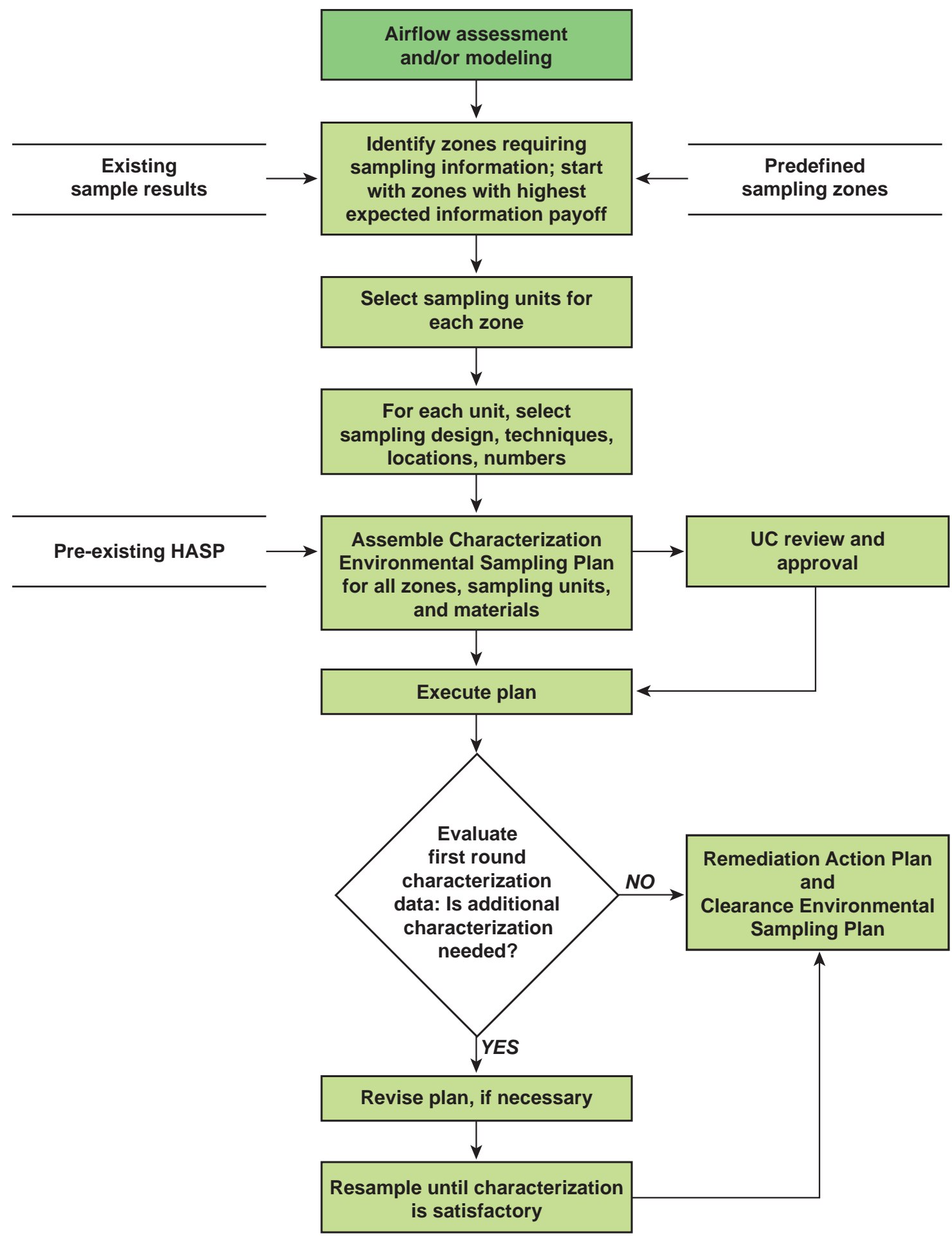

Figure 2-5. Major activities during the Characterization Phase. 


\section{CHARACTERIZATION}

For example, if any part of a concourse needs decontamination, then the entire concourse will be fumigated. It is unnecessary to determine that some portion of the concourse is contaminated and some portion is not. It might be desirable to know the extent of contamination with some degree of spatial precision for comparison with future clearance samples, or it might be helpful to learn that foot traffic transported the contaminant most of the way from one end to the other. But such additional detail is not necessary to support the decision of whether or not to fumigate the concourse. In other words, the question of extent is simplified: as soon as contamination is discovered anywhere in a potential fumigation zone, the decision is made, and sampling can stop.

In contrast, if there is reason to suspect the presence of one or more unknown hot spots, and it is judged worthwhile to bleach or tent and fumigate any hot spots prior to fumigating the entire concourse, then characterization sampling must be designed to yield a high likelihood of discovering the hot spots.

Thus, the most efficient characterization strategy can depend on the decontamination method. However, as characterization begins, it may not yet have been determined whether fumigation is necessary, or whether surface cleaning or decontamination will be done. In this case, the choice of decontamination strategy depends on the results of characterization. The interdependence of characterization and decontamination strategies suggests the following:

- An early characterization priority is to determine the likely decontamination method or methods. This determination, in turn, depends on agent properties and distribution.

- The entire cleanup effort should be viewed as an integrated process, rather than as strictly sequential steps.

The templates in Appendixes H and I provide a structure designed to help work through the airport area-byarea and zone-by-zone. The templates:

- Provide a mechanism for tracking objects and structures in an airport.

- Ensure that all types of items and structures are considered, even if not all are sampled. Potential sampling locations should be assessed for their likelihood to support necessary decisions or answer characterization questions.

- Save time by ensuring that sampling tasks are done systematically and thoroughly.

Using the templates, the EU must:

- Assess the likelihood of contamination in each zone.

- Decide what information is needed to support decisions in each zone.

- Decide how to sample to gather that information.

- Define decision points to cease characterization sampling if data warrant fumigation or complete removal of items.

Deciding that a zone needs decontamination is easier than deciding that it does not. Contamination in some parts of the airport, especially those remote from an overt release site, may seem unlikely. However, concluding that a zone does not need decontamination, when there is a possibility that it may have been contaminated, is equivalent to making a positive clearance decision during the Characterization Phase. If such a decision is under consideration, the EU must determine how much sampling support is necessary. 


\section{CHARACTERIZATION}

\subsubsection{Select Sampling Locations}

As described in Section 2.2.6 and Appendix D, the starting point for developing a detailed site- and eventspecific Characterization SAP should be a set of sampling zones and sampling units. A sampling unit is a subsection of a sampling zone - such as walls, floors, or furniture surfaces - that is sampled and evaluated collectively. With definitions of site-specific sampling units in hand, the EU or Sampling Group, or both, will be in a position to systematically consider each one in relation to the goals of characterization, and to choose an appropriate sampling design for each unit. The following determinations are key during characterization and in shaping the decontamination strategy:

- Deciding which areas of the airport require decontamination and which do not.

- Deciding what decontamination methods should be employed, given the type of agent and levels of contamination present.

- Deciding what materials, equipment, items, and surface types require decontamination in place versus removal and disposal, or removal and treatment.

- Identifying area(s) with the greatest predicted or confirmed agent concentrations.

- Identifying area(s) with the greatest potential for exposure to the public or airport workers.

The area(s) of greatest concentration and the area(s) of greatest potential for exposure may not be the same, and the potential for exposure may differ for different agents. For example, terminals, boarding gates, and baggage claims are likely to pose the greatest exposure potential, whereas the greatest agent concentration may be on exposed surfaces near the release point or within HVAC ducts. Both types of areas must be considered when developing sampling plans for characterization. All sources of information should be used, including:

- Locations of the release or releases, known or suspected.

- Estimates of the extent of contamination based on the operational mode of HVAC systems at the time of release, either known or suspected.

- Estimates of areas to which contamination may have been carried by foot traffic.

- Expected contaminant patterns from airflow model results, if available.

Potential sampling strategies include targeted, random, and probability-based methods. Targeted sampling can be used effectively when the source of contamination is known and supporting epidemiological and forensic data are available. This approach relies on existing information about the event to identify locations where additional sampling is expected to answer specific characterization questions. If nothing is known about where a release occurred, as might be the case for a covert release, or if sampling is done in zones where information about probable locations is weak or uncertain (for example, in zones distant from the release), a sampling grid or random approach could be used. A sampling grid could be designed to yield a high probability of discovering a hot spot of a given size. The TWG would need to specify the size of the grid and the probability of discovery. This kind of probability-based sampling is one way to decide how much characterization sampling is enough. For more information on sampling strategies, including specific suggestions for sampling locations, see Appendixes D and E. 


\section{CHARACTERIZATION}

\subsubsection{Select Sampling and Analysis Methods}

Personnel designated to perform sampling must be pre-trained in collection techniques. Knowing how to collect a sample useful for laboratory analysis requires specialized training.

Wipe samples can be used on hard, nonporous surfaces. Swab samples can be used to sample nooks, crannies, joints, and seams. Sample size should be maximized when possible. Use large-area samples (Buttner et al. 2004) with wipes or high-efficiency particulate air (HEPA) vacuuming to increase the ability to locate sparse contamination. HEPA vacuuming is useful as a sampling method on porous materials, such as carpets and certain types of furniture. This type of sampling can also be used on HVAC filters.

Characterization samples can be analyzed by methods based on the polymerase chain reaction (PCR), which yield rapid results. However, positive confirmation of viability requires use of bacterial culture methods at an LRN laboratory, which takes longer than PCR analysis. Rapid methods for determining BWA viability using combined PCR and culture methods are under development and may be available for future characterization needs through the LRNs. The LRN analytical laboratory should provide:

- Appropriate analytical procedures.

- $\quad$ Required detection limits.

- A documented quality assurance/quality control (QA/QC) program.

Appendix $\mathrm{C}$ contains additional information about sampling and analysis methods.

\subsection{Prepare Incident-Specific, Operational, Characterization Sampling and Analysis Plan}

Appendix I is a template for preparing an incident-specific, operational, Characterization SAP. The SAP document is developed and written by the EU, the Sampling Group in the Operations Section, or both. Upon completing the draft of the Characterization SAP, in which all goals are identified, an internal review is initiated. Upon approval of the plan by the $\mathrm{UC}$, characterization commences. There is no requirement that a single, written plan be generated for an entire airport. For example, SFO could have separate written plans for the International Terminal and each of the three other terminals. Whether or not such an approach is appropriate would depend on the specific details of an event. Separate plans would have to be written carefully, taking into account interconnected corridors and other potential pathways.

\subsection{Conduct Characterization Environmental Sampling; Evaluate Results}

The Sampling Group within the Operations Section of the Incident Command Structure implements the Characterization SAP. Upon completion of characterization activities, the first-round results are evaluated for completeness by the EU, with input from the TWG and with consultation by members of the ECC, as appropriate. If necessary, the Characterization SAP is revised, and additional characterization activities are recommended by the EU, and implemented as needed. 


\section{CHARACTERIZATION}

\subsection{Pre-Incident Planning}

Table 2-2 identifies the essential pre-incident planning activities related to site characterization.

Table 2-2. Summary of characterization-related actions to be taken prior to a BWA attack.

\begin{tabular}{|c|c|}
\hline $\begin{array}{c}\text { Responsible } \\
\text { Personnel }\end{array}$ & Pre-Incident Actions Related to Characterization \\
\hline $\begin{array}{l}\text { Airport authority } \\
\text { decision makers }\end{array}$ & $\begin{array}{l}\text { - Identify and document characterization resources shown in Table 2-1. } \\
\text { - Identify and document potential characterization, fumigation, and decontamination zones within } \\
\text { airport buildings. } \\
\text { - Identify and document sampling units. } \\
\text { - Identify and document areas at the airport that can be used or cleared for staging and storing } \\
\text { waste materials. } \\
\text { - Identify potential waste-disposal facilities. } \\
\text { - Make accessible all facility architectural and mechanical drawings. } \\
\text { - Periodically update HVAC blueprints and operating parameters. } \\
\text { - Periodically update building vulnerability assessments, and correct any deficiencies. } \\
\text { - Create new or review existing HASP. }\end{array}$ \\
\hline
\end{tabular}




\subsection{Summary}

Table 2-3. Summary of actions during the Characterization Phase showing the approximate sequence of events.

\begin{tabular}{|c|c|}
\hline Responsible Personnel & Action \\
\hline $\begin{array}{l}\text { Planning Section: Situation } \\
\text { Unit }\end{array}$ & $\begin{array}{l}\text { Compile all analytical and observational data and reports created during First Response, and } \\
\text { provide the information to the Environmental Unit. }\end{array}$ \\
\hline $\begin{array}{l}\text { Unified Command (or IC or } \\
\text { appropriate Unit Leader in a } \\
\text { large incident) }\end{array}$ & $\begin{array}{l}\text { Mobilize as necessary pre-identified resources for characterization activities, including: } \\
\text { - Laboratory Response Network (LRN) or other CDC-approved laboratories. } \\
\text { - Environmental sampling teams, decontamination and disposal resources, and PPE. } \\
\text { - Data management and documentation specialists. } \\
\text { - Air-dispersion modeling resources. } \\
\text { Activate TWG and ECC if desired; establish lines of authority and responsibilities. } \\
\text { Begin notifying resources for remediation, clearance, and waste management. }\end{array}$ \\
\hline $\begin{array}{l}\text { Site Safety Officer and } \\
\text { Medical Unit }\end{array}$ & $\begin{array}{l}\text { Create Health and Safety Plan (HASP), and determine appropriate PPE. } \\
\text { Vaccinate or provide antibiotics to appropriate cleanup and response personnel. }\end{array}$ \\
\hline Facility & $\begin{array}{l}\text { Provide detailed blueprints of areas of operation and HVAC systems to the Planning } \\
\text { Section, Documentation Unit. }\end{array}$ \\
\hline $\begin{array}{l}\text { Planning Section: } \\
\text { Environmental Unit }\end{array}$ & $\begin{array}{l}\text { Consider and recommend to the UC and/or Facility Manager immediate agent containment } \\
\text { and source reduction, if needed. } \\
\text { Implement any recommended agent containment and isolation actions. } \\
\text { Depending on actions completed during first response: } \\
\text { - Assess potential contaminant transport outside the facility (i.e., direct release, } \\
\text { rolling stock). } \\
\text { - Evaluate the need for air monitoring. } \\
\text { - Evaluate the need for conceptual or mathematical modeling. } \\
\text { With concurrence of Facility Manager, arrange to perform air modeling of movement of } \\
\text { BWA throughout facility to estimate initial extent of contamination. }\end{array}$ \\
\hline Operations Section & $\begin{array}{l}\text { Perform air monitoring, if needed, to detect BWA spread and potential exposure. } \\
\text { Ensure agent containment, and establish isolation for fumigation, if needed. }\end{array}$ \\
\hline $\begin{array}{l}\text { Planning Section: } \\
\text { Environmental Unit, with } \\
\text { input from Sampling Group } \\
\text { and Technical Working Group }\end{array}$ & $\begin{array}{l}\text { Develop a characterization sampling strategy to support cleanup activities. } \\
\text { Organize facility into characterization zones. } \\
\text { Select sampling locations for each zone. } \\
\text { Write an incident-specific characterization plan in which all goals are identified. }\end{array}$ \\
\hline Ops Section Chief and UC & Approve the characterization sampling plan. \\
\hline Ops Section: Sampling Group & Implement the characterization sampling plan. \\
\hline $\begin{array}{l}\text { LRN or other CDC-approved } \\
\text { laboratories }\end{array}$ & $\begin{array}{l}\text { Analyze samples to meet goals of characterization plan, including identifying characteristics } \\
\text { of the confirmed biothreat agent (e.g., survivability, toxicity, and ability to reaerosolize). }\end{array}$ \\
\hline $\begin{array}{l}\text { EU, with input from Technical } \\
\text { Working Group }\end{array}$ & $\begin{array}{l}\text { Evaluate results of characterization activities. } \\
\text { Consult with the ECC, as appropriate. } \\
\text { Recommend additional characterization activities to Operations Section, as needed. }\end{array}$ \\
\hline
\end{tabular}




\section{CHARACTERIZATION}

\subsection{Section 2 References}

Buttner, M. P., P. Cruz, L. D. Stetzenbach, A. K. Klima-Comba, V. L. Stevens, and P. A. Emanuel (2004), "Evaluation of the Biological Sampling Kit (BiSKit) for Large-Area Surface Sampling," Appl. Environ. Microbiol. 70(12), 7040.

Fitch, J. P. et al. (2002), Proc. IEEE 90(11), 1708.

USEPA (August, 2000), U.S. Environmental Protection Agency, Data Quality Objectives, U.S.EPA, EPA QA/G4.

Weis, C. P., A. J. Intrepido, A. K. Miller, P. G. Cowin, M. A. Durno, J. S. Gebhardt, and R. Bull (2002), "Secondary Aerosolization of Viable Bacillus anthracis Spores in a Contaminated U.S. Senate Office," JAMA 288 (22), 2853. 
Airport Biological Remediation Guidance 36 


\section{DECONTAMINATION}

\section{Decontamination}

Decontamination commences with source reduction, which includes separating salvageable and nonsalvageable items and actions such as pre-cleaning surfaces to reduce the contaminant load. The choice of decontamination technologies (Hawley and Kozlovac 2004) depends on the agent, nature and extent of contamination, and other site parameters identified during characterization. For nonpersistent biological agents, there may be sufficient experience within the realm of medical infection control and sanitation to inform decontamination decision-making. Potentially useful references include the CDC guidelines for Infection Control in Health-Care Facilities (2006) and the CDC Vessel Sanitation Program (2005) (both are available at www.cdc.gov/index.htm; click on Publications and Products).

For anthrax and other persistent BWAs, scenario-specific decontaminating reagents and delivery systems are selected, and all systems are pre-tested before carrying out chemical treatment(s). Site preparation for fumigation, if needed, includes sealing openings to prevent leaks and setting up equipment.

During implementation of the Remediation Action Plan (RAP), steps must be taken to prevent further environmental impacts. The effectiveness of any fumigation is assessed by monitoring four key process variables: temperature, relative humidity, fumigant concentration, and contact time, along with the results of biological indicators. Once specified criteria for any decontamination action have been met, final clearance can begin. Clearance is the process of clearing the facility for reopening to the public and is the subject of Section 4. Clearance activities usually include surface sampling and, in the case of B. anthracis or other aerosolizable agent, aggressive air sampling. Figure 3-1 summarizes the major activities during the Decontamination Phase.

The issue of "How clean is clean enough?" and the sampling and clearance methods by which the answer is determined are key to establishing effective and successful remediation (Raber et al. 2001). To date, the goal of cleanups involving B. anthracis spores is to ensure negligible residual exposure potential, demonstrated by no growth of $B$. anthracis cells in any clearance sample. Although clearance activities take place after decontamination actions are completed, clearance sampling should be planned at about the same time that decontamination is planned and before decontamination is actually done. In the end, remediation must be defensible to appropriate agencies and to the public. It is important to anticipate the issues of concern and to educate all relevant parties on the decontamination technologies selected and the criteria and clearance methods to be used.

An equally important consideration is to understand that no specific Remediation Action Plan can be developed for an airport in advance of an attack. Specific choices depend on the nature of the BWA, location of release, extent of contamination, and all other parameters that are the focus of characterization. Thus, this section addresses the nature of actions to be taken and decisions to be made in devising an optimal remediation approach. Details on specific decontamination reagents, techniques, and applications are provided in Appendix F. 


\section{DECONTAMINATION}

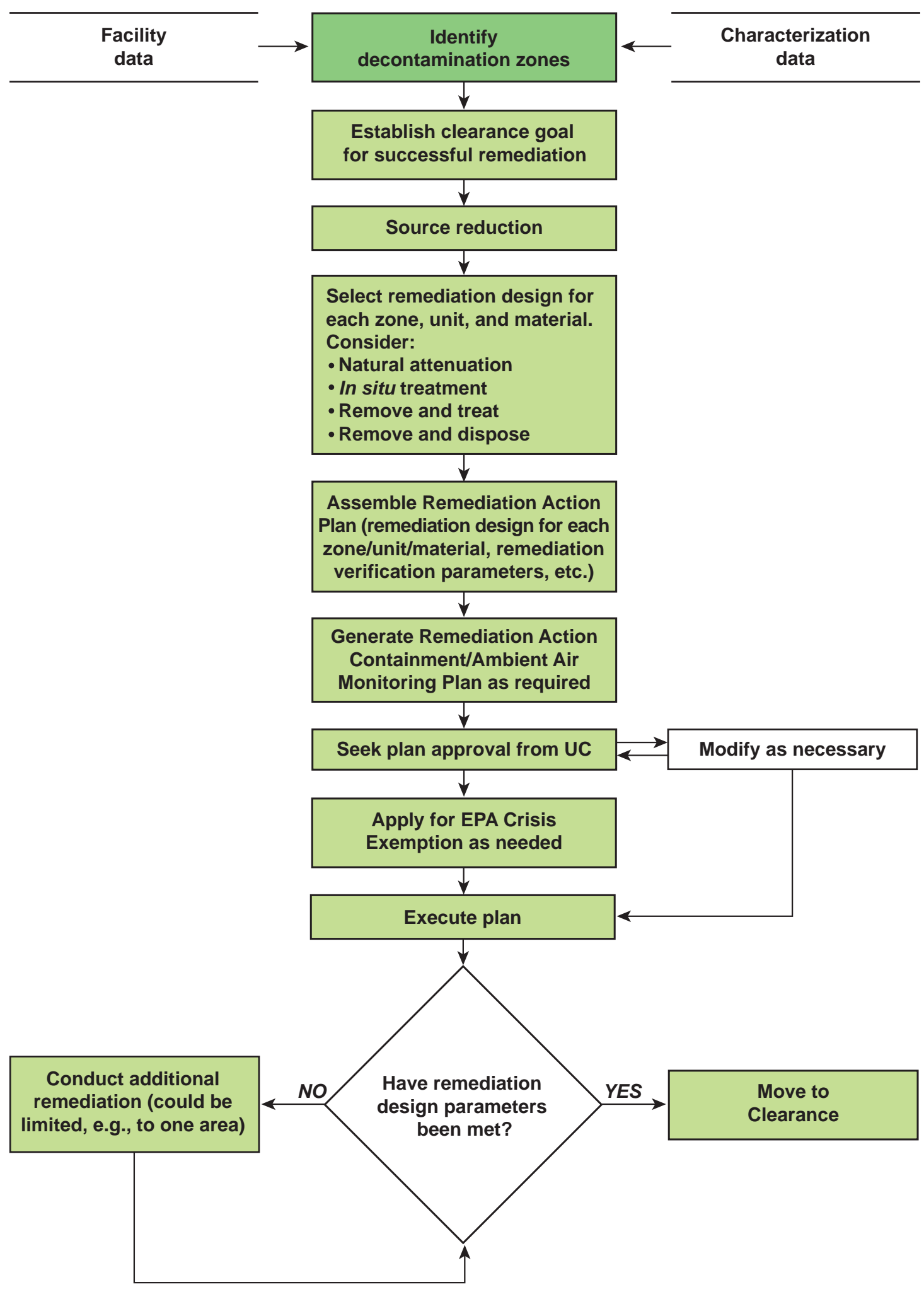

Figure 3-1. Major activities during the Decontamination Phase. 


\section{DECONTAMINATION}

\subsection{Evaluate Decontamination Capabilities}

Several decisions must be made by airport personnel well in advance of the need to implement a scenariospecific Remediation Action Plan in response to a terrorist attack. Such decisions regarding decontamination capabilities include:

- The choice of in-house equipment that could be used or upgraded to support some types of decontamination, such as surface decontamination.

- Location and number of staging areas or warehouses for equipment and supplies.

- Extent and types of decontamination supplies to store.

- Selection of potential contractors to employ as members of the decontamination team.

- Identification of potential waste-disposal facilities.

For more details on these issues, refer to Appendix F. Keep in mind that decontamination-related decisions can have a major impact on waste-disposal costs and can present significant nontechnical (e.g., legal and regulatory) challenges at the time that disposal of waste takes place. Table 3-1 lists the types of resources (agencies, teams, and technical contacts) that should be identified by airport personnel in advance.

Table 3-1. Site decontamination resources. (Airport to fill in contact information.)

\begin{tabular}{|l|l|l|}
\hline \multicolumn{1}{|c|}{ Resource } & \multicolumn{1}{c|}{ Contact } & Phone \\
\hline Facility engineering and construction team(s) & & \\
\hline $\begin{array}{l}\text { Decontamination and fumigation team (may } \\
\text { include decontamination reagent suppliers) }\end{array}$ & & \\
\hline $\begin{array}{l}\text { National Decontamination Team, On-Scene } \\
\text { Coordinators, and National Homeland Security } \\
\text { Research Center (USEPA) }\end{array}$ & & \\
\hline Primary LRN analytical laboratory & & \\
\hline Secondary LRN analytical laboratory & & \\
\hline $\begin{array}{l}\text { Sampling team(s) and contractor(s) for placement, } \\
\text { collection of biological indicators }\end{array}$ & & \\
\hline Centers for Disease Control and Prevention (CDC) & & \\
\hline Personal protective equipment (PPE) rentals & & \\
\hline State solid-waste management division & & \\
\hline Local wastewater treatment facility & & \\
\hline
\end{tabular}

\subsection{Establish Overall Goal for Successful Decontamination}

Natural attenuation may be adequate for certain, nonpersistent to moderately persistent BWAs (a range of highly virulent viruses and nonspore forming bacteria), but it will not be adequate to eliminate risks 
to humans from highly persistent, spore-forming pathogens, such as B. anthracis. Thus, decontamination options must be evaluated in terms of the specific agent involved, how it is prepared or modified, and other site-specific considerations. Before selecting a decontamination technique, however, a major consideration is to define the overall goal for determining whether decontamination is successful. Ultimately the UC approves the overall decontamination goal. Unfortunately, decision makers cannot refer to established cleanup levels for biological agents because they do not exist. Because of this important information gap, it is necessary to rely on other precedents.

For the anthrax cleanups to date, decontamination was not considered successful until there was no growth of B. anthracis from any clearance environmental sample (U.S. General Accounting Office 2003). In 2003, the National Academy of Sciences was commissioned to review all available data and recommend a cleanup level for use in remediating facilities contaminated with BWAs, such as B. anthracis. This study concluded that there is currently no scientific basis for establishing a level of residual contamination that can be safely left behind. Dose-response relations for specific BWAs are not sufficient to understand infectivity and virulence with confidence. Furthermore, insufficient information is available on which to base "safe" numbers of residual BWAs after decontaminating a facility (NRC 2005).

There is research underway that may help establish a scientific basis for setting a decontamination goal other than "no growth." Future decisions on decontamination effectiveness will likely factor in process parameters (e.g., temperature, humidity, contact time, etc.), as well as better data on agent characteristics/behavior (both indoors and outdoors), improved sampling strategies, and new methods of exposure and risk assessment.

\subsection{Evaluate Natural Attenuation}

Under certain circumstances, it is possible that natural attenuation (simply waiting) could be adequate to eliminate acute and chronic impacts from some types of BWAs, especially agents that are present as vegetative cells or are not viable for more than a few hours outside a host. If such an agent were the one of concern, then many of the Decontamination Phase activities described in this section may not be required, and re-entry and resumption of airport operations could be allowed after a suitable time and limited clearance environmental sampling. Natural attenuation should be considered as a decontamination option within a risk-based framework, but in the case of spore-forming organisms, such as B. anthracis, natural attenuation is probably not an option, especially for indoor releases, because the spores remain viable for long periods in the environment. It is also important to consider whether a naturally nonpersistent agent might have been enhanced to be more stable in the environment. The assumption that an agent is not persistent should be verified by environmental sampling.

\subsection{Isolate Decontamination Areas}

Isolation of contaminated areas from the remainder of the airport begins during first response and continues during characterization (see Section 2.2.7). Any existing isolation needs to be reviewed for adequacy if fumigation with gaseous or vaporous decontamination reagents is to be used.

Any isolation put in place during first response or characterization was probably not done with fumigation in mind. In some instances, the isolation already in place may be suitable for fumigation. However, if the volume of isolated space exceeds the volume capacity of a fumigation technology, then either another technology needs to be selected, or the isolated areas must be subdivided into smaller volumes. Seals can be smoke tested for leak-tightness. Appendix F provides more details on isolation barrier technology. 


\section{DECONTAMINATION}

Negative air units (NAUs), also known as negative air machines, can be used for containment and are used for isolation. NAUs subject the area to a slightly negative pressure to ensure that the agent and fumigant remain in the contamination zone. NAUs can be used in each area to be decontaminated.

\subsection{Develop Decontamination Strategy}

After sufficient information is available about the areas and types of materials requiring decontamination, the decontamination planning activities can begin. This effort culminates in the preparation of an incidentspecific RAP by the responsible organization, with advice from the TWG. An incident-specific RAP describes a decontamination strategy that consists of several elements including, but not limited to, the following:

- What airport facilities and areas need to be decontaminated.

- What materials and structural components are to be decontaminated in situ, or removed for treatment and either reused or disposed.

- To what extent removed items will be decontaminated prior to disposal, and how and where such items will be decontaminated and disposed.

- How and where other types of wastes will be disposed.

- Which decontamination technologies are to be used.

- Which personnel and teams are responsible for the decontamination tasks.

Recall that the first element in the list above (areas requiring decontamination) will have been determined after sampling and analysis are completed during characterization. Furthermore, if decontamination capabilities have been established in advance (as is recommended in Section 3.1), then the RAP can be prepared more rapidly. The following sections describe in more detail the remaining, necessary components of a decontamination strategy.

\subsubsection{Perform Source Reduction}

Initial source reduction commences during characterization, as discussed in Section 2.2.5. The objective of source reduction is to decrease the amount of contamination in a facility before the main decontamination activity. Prior to decontamination, decisions need to be made concerning what materials and structural components will be decontaminated for reuse either onsite or offsite, and what will not be reused, but will be packaged, with the package decontaminated externally, and removed for disposal either as waste or through recycling. Nonessential items removed for disposal are treated very differently from essential items removed for offsite treatment and returned for reuse. A facility's structural components and essential items are typically decontaminated for reuse, whereas removable materials (flooring, false ceilings, acoustic tiles, and low-end computers) can be packaged, the package decontaminated externally, and transported for disposal. A cost-benefit analysis should be incorporated in the decision process related to retention versus disposal of items, or costs can rise unnecessarily. Source reduction during the Decontamination Phase is performed by the Operation Section's Decontamination Group.

For facilities at which fumigations are to be conducted, source reduction of materials that will remain onsite (e.g., equipment) and structural elements of the facility may include prior treatments with HEPA vacuuming 
and surface treatment (such as with amended sodium hypochlorite solution). HEPA vacuuming is especially useful on porous materials. In addition, it removes dirt and other debris that can reduce the effectiveness of subsequent decontamination by surface reagents or fumigation. An advantage of HEPA technology is that there is little potential for collateral damage. The technology can only be used to remove or reduce surface contamination and may not be appropriate for the internal workings of sensitive electronic equipment. The potential for spreading contamination exists if the exhaust is allowed to stir the air in a contaminated area. Details on the use of HEPA vacuuming are in Appendix F.

Sensitive equipment, such as computers, electronic and electrical circuit boards, high-voltage power lines, and electronic control panels, are not amenable to aqueous decontamination systems. The problem of sensitive equipment is discussed below and in more detail in Appendix F. Contaminated planes are best removed from service initially, with determination of disposal versus decontamination and reuse made at a later date.

Removal of any contaminated material will require transportation to an approved treatment and disposal facility. The Department of Transportation and individual states have many requirements for pre-treating and packaging materials prior to leaving a contaminated facility, labeling packages for transport, and transporting material contaminated with infectious agents to approved facilities. The separate category of personal or valuable items that could be removed for offsite decontamination is discussed below and in Appendix F. Additional details related to waste treatment and disposal are provided in Appendix G.

\subsubsection{Select Decontamination Technologies}

A large airport contains many different types of areas that may need to be decontaminated. Areas range from large, open atriums typically found in terminal buildings and ticket counter check-in areas; to long, relatively narrow boarding gates; to a variety of concessions that include restaurants and retail outlets. Most of the areas are enclosed or semi-enclosed spaces. In addition, airports have specialized equipment, such as baggage handlers and large, industrial air handling units (AHUs) that facilitate the spread of spores and potentially complicate cleanup efforts. A substantial portion of the baggage handling system is located outside on the runway level in either completely open or minimally semi-enclosed spaces. Sophisticated computers that control baggage flow, gate schedules, and passenger flow, along with complex security screening machines, may need to be decontaminated.

Because of the complex landscape, four types of decontamination technologies should be considered for contaminated facilities and their contents:

- Liquid or semi-liquid reagents to decontaminate exposed nonporous and porous surfaces, respectively.

- Gaseous or vaporized reagents to decontaminate difficult-to-reach, porous and nonporous surfaces, including HVAC and AHU systems.

- Technologies to decontaminate sensitive electronic equipment.

- Technologies to decontaminate small, personal, or valuable items, such as baggage and artwork

No existing decontamination reagent is currently registered by the USEPA specifically for inactivation of $B$. anthracis spores. Therefore, for each specific use of a selected reagent to decontaminate a facility contaminated by $B$. anthracis, an emergency exemption must first be obtained from the USEPA. The USEPA has the authority to issue such exemptions when emergency conditions exist. Most exemptions require an 


\section{DECONTAMINATION}

application and quick review from EPA before they can be issued. Where the discovery of an emergency condition and the need to use a pesticide require quicker action, USEPA would expect to issue a crisis exemption. Details concerning the crisis exemption are in Appendix F.

\subsubsection{Liquid or Semi-Liquid Decontamination Reagents for Surfaces}

Liquid or semi-liquid reagents are the most common decontamination products that can be applied to exposed surfaces. If the BWA is a low-grade preparation that is not readily aerosolized, then surface decontamination may be all that is necessary for decontamination. If aerosolizable material is present (Weis et al. 2002), liquid oxidizing reagents or HEPA vacuuming can be used to reduce the level of contamination and pre-clean surfaces prior to fumigation. Several organizations have been granted crisis exemptions to use certain decontamination agents. The reagents include:

- A 10:1 dilution of 5.25\% to $6 \%$ bleach [5,250 to 6,000 parts per million (ppm)] adjusted to $\mathrm{pH} 7$. The "amended bleach" solution is created by adding one part acetic acid, to one part $5.25 \%$ to $6 \%$ bleach, to eight parts water. A 60-minute wet contact time is required.

- Liquid chlorine dioxide, generated onsite from sodium chlorite (500-ppm $\mathrm{ClO}_{2}$ ). A 30-minute wet contact time is required.

- Peracetic-acid/hydrogen-peroxide-based biocides. Brands include Spor-Klenz RTU, Oxonia Active, KX-6049, and Actril Cold Sterilant as ready-to-use solutions. A 15- to 20-minute wet contact time is required.

- Hydrogen peroxide biocide. Virex STF is a ready-to use-solution. A 20-minute wet contact time is required.

Two semi-liquid products (one gel and one foam) have been tested in the laboratory but have not been granted crisis exemptions for use in B. anthracis spore cleanups. An example is DF 200, which contains hydroperoxide and hydroperoxycarbonate anions plus a surfactant, fatty alcohols, and water-soluble polymers. This two-part system, requires a 3-hour wet contact time. It is commercially available through Modec and Enviro-Foam, and is currently used by the Department of Defense. SFO has outfitted a decontamination bus that can use DF 200 in first response and remediation actions, if appropriate.

No liquids or semi-liquids are registered under the Federal Insecticide, Fungicide, and Rodenticide Act (FIFRA) for use against B. anthracis spores. The advantages and disadvantages of each are shown in Table 3-2. Appendix F provides more information on these and other surface decontamination reagents and their effectiveness.

\subsubsection{Gaseous or Vaporized Decontamination Reagents}

Depending on the nature and quality of the preparation, some BWAs may be easily transported through the air. The small size of spore-formers such as B. anthracis (approximately 1 to 3 microns) means they can easily enter small areas, such as cracks, corners, and hard-to-reach areas. If the spores were refined and mixed with other materials, they might be easily reaerosolized and could pose additional threats in terms of human exposure. Surface decontamination reagents are not likely to reach all areas where spores could potentially accumulate. If characterization sampling indicates that the spores are refined, or there is epidemiological evidence of human inhalational anthrax associated with a contaminated site, then it would be necessary to use a gaseous or vaporized decontamination reagent that can reach all areas of a contaminated zone. 


\section{DECONTAMINATION}

Table 3-2. Advantages and disadvantages of selected liquid or semi-liquid decontamination reagents.

\begin{tabular}{|c|c|c|}
\hline Decontamination option & Advantages & Disadvantages \\
\hline Liquid chlorine dioxide & $\begin{array}{l}\text { Previous crisis exemption issued for } \\
\text { inactivation of } B \text {. anthracis. } \\
\text { Contact time only } 30 \text { minutes. }\end{array}$ & $\begin{array}{l}\text { Requires onsite generation system. } \\
\text { Gaseous phase is acutely toxic. } \\
\text { Only effective on hard, nonporous surfaces. }\end{array}$ \\
\hline $\begin{array}{l}\text { Spor-Klenz, Oxonia Active, } \\
\text { KX-6049, Actril Cold } \\
\text { Sterilant, Virex STF }\end{array}$ & $\begin{array}{l}\text { Previous crisis exemptions issued for } \\
\text { inactivation of } B \text {. anthracis. } \\
\text { Ready-to-use, water-based formulations. } \\
\text { Contact time only } 10 \text { to } 20 \text { minutes. }\end{array}$ & Only effective on hard, nonporous surfaces. \\
\hline $\begin{array}{l}\text { 10:1 diluted, } \mathrm{pH} 7 \text { sodium } \\
\text { hypochlorite } \\
(5,250 \text { to } 6,000 \mathrm{ppm})\end{array}$ & $\begin{array}{l}\text { Previous crisis exemption issued for } \\
\text { inactivation of B. anthracis. } \\
\text { U.S. Army standard for CWAs. } \\
\text { Easily dispensed by spraying, mopping, or } \\
\text { scrubbing. } \\
\text { Inexpensive and widely available. }\end{array}$ & $\begin{array}{l}\text { Not suitable for sensitive equipment, such as } \\
\text { computers; corrosive to metals. } \\
\text { Only effective on hard, nonporous surfaces. } \\
\text { Long contact time required ( } 60 \text { minutes), and } \\
\text { limited shelf life. }\end{array}$ \\
\hline SNL Decon Foam DF 200 & $\begin{array}{l}\text { Effective on most substrates. } \\
\text { Low toxicity. } \\
\text { Good shelf life. } \\
\text { Commercially available }\end{array}$ & $\begin{array}{l}\text { Crisis exemption for DF } 100 \text { revoked. } \\
\text { Reformulated as DF } 200 . \\
\text { Somewhat expensive. } \\
\text { Long contact time required ( } 3 \text { hours). } \\
\text { Binary system requires mixing. } \\
\text { 6- to 10-hour wait for foam collapse. } \\
\text { Messy to apply and clean up. Leaves oily } \\
\text { residue; requires cleaning. }\end{array}$ \\
\hline
\end{tabular}

Table 3-3 lists the principal advantages and disadvantages of some of the most useful gaseous and vaporized decontamination reagents. None of these gases or vapors is registered for inactivation of $B$. anthracis spores in buildings or on their contents. Therefore, a crisis exemption must be obtained from the USEPA for each specific use of these gas or vapor products. Appendix F provides more information on the reagents and their advantages and disadvantages. Different gaseous and vaporized decontamination reagents may require different amounts or types of materials to be removed before fumigation. The choice of reagents for large-area fumigation would depend on the size of required staging areas and waste storage areas as well as disposal costs.

Three fumigants, ethylene oxide, methyl bromide, and paraformaldehyde, could be used for decontaminating small or critical items that can be handled in a small-scale, batch mode at an offsite treatment facility. Examples of such items include artwork, computers, personal items, and luggage. Although they are highly effective, they must be carefully controlled because they 


\section{DECONTAMINATION}

Table 3-3. Advantages and disadvantages of gas and vapor decontamination reagents.

\begin{tabular}{|c|c|c|}
\hline Decontamination option & Advantages & Disadvantages \\
\hline $\begin{array}{l}\text { Vaporous hydrogen peroxide } \\
\text { (VHP) }\end{array}$ & $\begin{array}{l}\text { Registered by USEPA as a sterilant. } \\
\text { Crises exemptions issued for inactivation of } \\
\text { B. anthracis. } \\
\text { Used in small-scale commercial and } \\
\text { industrial settings. } \\
\text { Benign byproducts; no toxic residues. }\end{array}$ & $\begin{array}{l}\text { Process is currently limited to a volume of } \\
250,000 \mathrm{ft}^{3} \text {. } \\
\text { Degradation of VHP reagent through contact } \\
\text { with galvanized steel and porous surfaces. } \\
\text { Limited vapor penetration capability: most } \\
\text { building contents may need to be removed, } \\
\text { treated, and either disposed or reused. }\end{array}$ \\
\hline Chlorine dioxide & $\begin{array}{l}\text { Registered by USEPA as a sterilant. } \\
\text { Previous crisis exemptions issued for } \\
\text { inactivation of B. anthracis. } \\
\text { No limit on volume of space } \\
\text { decontaminated. } \\
\text { Use of tenting structures provides faster, less } \\
\text { expensive approach to containment. } \\
\text { Good penetration capability of gas allows } \\
\text { for much of building's contents to be left in } \\
\text { place, thereby reducing waste for disposal. }\end{array}$ & $\begin{array}{l}\text { Logistics are complex for large-scale } \\
\text { decontamination. } \\
\text { Degradation of } \mathrm{ClO}_{2} \text { through contact with } \\
\text { porous surfaces. } \\
\text { Potential material-compatibility issues with } \\
\text { certain metals. } \\
\text { Potential fine residue (nontoxic). } \\
\text { Sensitive to UV. }\end{array}$ \\
\hline Ethylene oxide & $\begin{array}{l}\text { Highly effective and may only be used on a } \\
\text { small scale. } \\
\text { Crisis exemptions issued for small-scale, } \\
\text { batch use. }\end{array}$ & $\begin{array}{l}\text { Difficult to neutralize on a large scale. } \\
\text { No previous crisis exemption for large scale } \\
\text { because of explosivity and other hazard issues. }\end{array}$ \\
\hline Methyl bromide & $\begin{array}{l}\text { Highly effective on any scale. } \\
\text { Previous crisis exemptions issued for } \\
\text { experimental fumigations. } \\
\text { Has been used at large scale for termite and } \\
\text { agricultural purposes. } \\
\text { Can be done by tenting. } \\
\text { Does not require humidity control. } \\
\text { Economical. } \\
\text { High penetration capability of gas allows } \\
\text { for most of building's contents to be left } \\
\text { in place, thereby minimizing waste for } \\
\text { disposal. }\end{array}$ & $\begin{array}{l}\text { Major uses being phased out under Montreal } \\
\text { Protocol, but critical uses continue. } \\
\text { Long contact time of } 24 \text { to } 48 \text { hours. } \\
\text { Difficult to neutralize on a large scale. }\end{array}$ \\
\hline Paraformaldehyde & $\begin{array}{l}\text { Highly effective, and best used on a small } \\
\text { scale. } \\
\text { Previous crisis exemption for confined } \\
\text { spaces. } \\
\text { No materials compatibility problems. } \\
\text { Good penetration capability of gas allows } \\
\text { for much of building's contents to be left in } \\
\text { place, thereby reducing waste for disposal. }\end{array}$ & $\begin{array}{l}\text { No previous crisis exemption for large scale, } \\
\text { but USAMRIID has decontaminated buildings. } \\
\text { Difficult to neutralize on a large scale. } \\
\text { Minor residues must be removed by washing. } \\
\text { Potential stakeholder resistance because of } \\
\text { possible carcinogenicity. }\end{array}$ \\
\hline
\end{tabular}


are toxic and can be difficult to neutralize on a large scale. A scrubbing process is available for methyl bromide, but it is on a small scale and has limited effectiveness. A neutralization process is available for formaldehyde, namely reaction with ammonium bicarbonate. Ethylene oxide is not a large-area fumigation technology, but its use for decontamination in a sterilization (AAMI 1995) chamber is a treatment of choice for essential items.

\subsection{Monitoring the Effectiveness of the Fumigation Process}

The effectiveness of the fumigation process is evaluated on the basis of two factors. First, four key process variables are monitored throughout fumigation. The four variables are temperature, relative humidity, fumigant concentration, and contact time. Each must be kept within a specified range. Temperature and relative humidity are recorded continuously during fumigation. Fumigant gas or vapor concentration is always monitored by real-time chemical sensors or chemical analysis of manually collected samples; it is sometimes monitored with chemical-sensitive paper as well. Second, the effectiveness of fumigation is confirmed through use of biological indicators (BIs). The indicators consist of spores of a species related to $B$. anthracis that are not pathogenic to humans. BIs are placed on strips that are set in various locations within the fumigation volume as a method of verifying sporicidal conditions. Appendix F contains more detail on procedures for verifying fumigation.

\subsection{Ambient Air Monitoring}

Use of gaseous or vaporous fumigants requires air monitoring outside a facility to ensure that fumigant does not escape the facility in concentrations that may be a hazard to workers or the surrounding population. As part of the crisis exemption application, the USEPA requires a written ambient air monitoring plan (AAMP). The EU prepares the AAMP and submits this plan to the USEPA. Appendix F contains more detail on the AAMP. The RAP template (Appendix J) contains a section for ambient air monitoring.

\subsubsection{Decontamination of Sensitive Electronic Equipment}

Decontamination technology for sensitive equipment, such as expensive CTX machines, is a major concern for airports. Such technology is also required for unprotected electronic and electrical components that may be associated with computer systems, electronic circuit boards, baggage handling systems, and security systems. Aqueous-based decontamination systems are inappropriate, as would be any procedure that corrodes, leaves a residue, or chemically reacts with component parts. However, aqueous systems could be used to decontaminate the outside cases of sealed electronic equipment. If the electronics are truly sealed, and there are no cooling fans drawing outside air into the equipment, it may not be necessary to decontaminate internal components, because, in theory, they should not be contaminated.

If the internal parts of machinery and equipment are sampled and found to be contaminated, then a gaseous or vaporized product could be selected. A crisis exemption for paraformaldehyde, methyl bromide, ethylene oxide, vaporous hydrogen peroxide, and chlorine dioxide could be requested for this purpose. Use of vaporous hydrogen peroxide may require that equipment be opened up completely; it is less penetrative than chlorine dioxide. However, vaporous hydrogen peroxide is used in many pharmaceutical applications on sensitive electronic equipment. A consideration in the use of chlorine dioxide is the potential impacts of salt residue. The Department of Defense will not use chlorine dioxide on aircraft for this reason. Large airports should identify those pieces of electronic equipment deemed absolutely necessary to the operation of the facility, and plan for either decontamination or replacement in the event of contamination. 


\subsubsection{Decontamination of Personal or Valuable Items}

Sterilants and irradiation chambers can be used to decontaminate personal or valuable items removed from a facility. Irradiation is used to treat some Government mail, although paper may become brittle and plastic items (such as credit cards and cellophane) melt. Similarly, using irradiation chambers to inactivate spores may destroy some items, especially plastics, and may not penetrate sufficiently if the item has significant bulk. In addition, sterilants may be used for smaller pieces of sensitive electronic equipment. Chemicals such as paraformaldehyde, methyl bromide, ethylene oxide, vaporous hydrogen peroxide, or chlorine dioxide may be used to kill spores on discrete items placed in a sterilization chamber. Adequate aeration of items after treatment is required to remove residual amounts of sterilant and any toxic by-products that may have formed. Appendix F contains more detail on the use of sterilants and irradiation chambers.

\subsubsection{Evaluate Potential Environmental Impacts}

Decontamination actions must be implemented in a manner that prevents any release of harmful reagents, contaminants, or byproducts to the environment. Liquid waste streams resulting from the use of a decontamination reagent or removal of the decontamination reagent from airport surfaces must be managed to prevent release into the airport drainage system and ultimately into nearby rivers or other surface water. Agents that are acidic or caustic, or that have strong oxidizing properties, should be contained. If appropriate, and permission is given by the local sanitary agency, such agents can either be discharged to the sanitary sewer or handled otherwise as a special waste. Airports must identify the location of drains or other connections that would provide a route for materials to enter the environment. Where such exit routes exist, measures should be taken to protect the exits, such as plugging storm drains prior to the start of surface decontamination activities or containing flows from the decontamination area before discharge to the environment. The resultant waste materials can be removed using wet-dry vacuums, mops, or wiping down surfaces. If a centralized containment or staging area has been established, the materials can be rinsed into the containment area where wastewater may need to be treated, then characterized, pumped, and properly disposed. The need for treatment depends on local regulatory agencies, which should be contacted early in the remediation process.

Taking such measures before waste is released to the environment can potentially save large expenditures of resources that would otherwise be required to remediate contaminated areas once a waste containing biological agent is released into the environment. If a release does occur, applicable regulatory requirements must be met in mitigating any damage that may have occurred to the environment.

Fumigation technologies have the potential for emission of fumigants into the air. An Ambient Air Monitoring Plan (AAMP) is required to ensure that such releases do not occur.

\subsubsection{Evaluate Waste Disposal Options}

Decontamination decisions have a major impact on disposal decisions, and vice versa. Waste disposal is typically regulated by the solid-waste management division of a state's relevant environmental protection department, and the waste disposal facility must voluntarily accept the waste through a contractual arrangement. Both of those entities are important stakeholders. It is generally easier to dispose of wastes in facilities within the same state in which an airport is located, as opposed to another state; however, optimal disposal facilities for certain waste streams may not be present in a particular state.

Before discussing waste disposal with potential disposal facilities, it is important to have at least a rough idea of the quantities and characteristics of materials destined for disposal. An important aspect of the waste 


\section{DECONTAMINATION}

disposal process is the regulatory category into which waste falls. The categorization of types of wastes varies from state to state, but materials from a BWA remediation might be categorized as regulated medical waste or as a special waste. New York State has begun a process of defining bioterrorism waste from a regulatory perspective.

A disposal plan should be developed at the same time as decontamination planning. The disposal plan should estimate types and amounts of wastes, transportation needs, and costs. It should also describe any clearance sampling that will be done at disposal sites, as well as long-term monitoring requirements, if necessary. Appendix G and Section 6 of Appendix J provide a framework from which to start.

\subsection{Prepare Remediation Action Plan and Related Documents}

The EU or Decontamination Group, or both, prepare an incident-specific RAP detailing the decontamination methods to be used. The Operations Section Chief reviews, and the UC approves, this plan. The overall RAP is implemented in a series of daily Incident Action Plans (IAPs) as defined in the National Response Framework. The template in Appendix J can facilitate preparation of the RAP. Depending on the event, not all sections in Appendix $\mathrm{J}$ will be required. The RAP must be approved by the USEPA if the bioterrorism agent is B. anthracis spores and if a crisis exemption is required. For any other bioterrorism agents, consult the USEPA about whether a crisis exemption is required.

If the Remediation Action Plan calls for fumigation, then the plan should also be accompanied by an AAMP and a SAP, which includes characterization sampling and post-decontamination sampling. (Post-decontamination sampling is also needed for decontamination that does not involve fumigation; items treated with liquids and semi-liquids also need to be sampled and cleared.) For biological agent decontamination, the Remediation Action Plan together with the AAMP and SAP are used to obtain a crisis exemption from the USEPA.

If pesticide products become registered for inactivation of B. anthracis in the future, the three plans will likely be required by the product's labeling, but the plans would no longer be submitted to the USEPA for approval; instead, the Incident Commander would approve them. If fumigation is used, an Emergency Response Plan is also recommended, although such a plan is not required to obtain a crisis exemption. The Site Safety Officer develops the Emergency Response Plan to address potential, uncontrolled fumigation releases (e.g., from explosion, fire, or hurricane).

\subsection{Perform Site Preparation for Fumigation}

The Operations Section's Decontamination and Sampling Groups perform all site preparations specified in the RAP. Site preparation before decontamination using fumigation includes many activities, such as:

- Subdividing space with temporary walls.

- Sealing all leaks and openings, and testing for leaks.

- Installing and testing chemical-generation systems.

- Installing and testing chemical, temperature, and humidity monitoring systems.

- Installing and testing NAUs and air-scrubbing systems.

- Commissioning new equipment.

- Testing low-level fumigation.

The details of site preparation are event- and airport-specific. 


\section{DECONTAMINATION}

\subsection{Perform Decontamination}

After the RAP, SAP, and AAMP are completed, the UC approves the three documents (RAP, SAP, and AAMP), and the EU submits them to the USEPA along with a request for a crisis exemption. Alternatively, the EU can submit drafts of the three documents to the USEPA for a preliminary review to ensure the information is complete before final submission together with a formal exemption request.

Upon receipt of the crisis exemption from the USEPA, the designated decontamination contractor(s) and trained decontamination personnel carry out the decontamination, with oversight by the Operations Section's Decontamination Group. Specific remediation actions cannot be suggested for an airport in advance of an attack because the details of remediation activities are specific to the agent, site, and event. Following decontamination activities, the EU and the Decontamination Group, with input from the TWG, evaluate the results for completeness and to ensure that process criteria have been met (see Section 3.9). The ECC is consulted during the evaluation, as necessary. The EU, Decontamination Group, or both, may recommend additional decontamination activities, if warranted.

\subsection{Verify Decontamination}

Decontamination processes are monitored as they are being carried out and then evaluated as to whether they have been conducted successfully. For liquid decontamination chemicals applied to hard, nonporous surfaces, the efficacy of a product depends on whether the minimum product concentration and contact time specified on the product label — or specified in the crisis exemption issued by the USEPA - have been attained. Records of key parameters must be kept to show that criteria for a successful decontamination process were met.

For gaseous or vaporized decontamination chemicals, the key parameters of temperature, relative humidity, chemical concentration, and contact time are monitored and recorded for each of the four phases of the fumigation process - (de)humidification, conditioning, decontamination, and aeration. Maintaining the variables in prescribed ranges throughout fumigation is one measure of the efficacy of the process. In addition, biological indicators (BI), which contain nonpathogenic (surrogate) spores that are most resistant to a specific fumigant, are used during fumigation to provide additional evidence that the fumigation was effective in reaching all areas to which the biological agent may have migrated.

In general, BIs are an accepted way to monitor the effectiveness of the fumigation process because they directly determine whether the most resistant microorganisms (e.g., Geobacillus or Bacillus species) to a specific fumigant were killed. In addition, BIs provide some indication of whether the four key process variables have remained in prescribed ranges throughout the fumigation process. Because spores used in BIs are usually more resistant and typically present in greater numbers than the biological agent itself, inactivation of BIs indicates that the pathogen causing the contamination has also been inactivated. A variety of different uncombined spore preparations can be used, including Bacillus atrophaeus and Geobacillus stearothermophilus. A specific concentration of viable spores (generally one million) is usually dried on filter paper (spore strips) or stainless-steel coupons and placed in a protective glassine or Tyvek pouch.

BIs are placed in various locations at a minimum frequency of one per 100 square feet of floor space, as specified in the Clearance SAP. Placing BIs in locations of known or suspected contamination and in spaces hard to reach by the fumigant is standard practice. Positive and negative control BIs are also employed. After fumigation is complete, treated and control BIs are sent to an analytical laboratory with demonstrated experience in analyzing BIs from biomedical sterilization and other relevant fumigation processes. They are 


\section{DECONTAMINATION}

then incubated to determine spore viability.

When process parameters are met, and all spores on the BIs have been killed, the fumigation can be judged effective. If a very small percentage of BIs are positive, further environmental sampling is performed at locations of the positive BIs. However, the overall criterion for success of a remediation is usually judged by environmental clearance sampling, as described in Section 4.

\subsection{Pre-Incident Planning}

Table 3-4 identifies essential pre-incident planning activities related to site decontamination.

Table 3-4. Summary of decontamination-related actions to be taken prior to a BWA attack.

\begin{tabular}{|c|c|}
\hline $\begin{array}{c}\text { Responsible } \\
\text { Personnel }\end{array}$ & Pre-Incident Actions Related to Decontamination \\
\hline $\begin{array}{l}\text { Airport authority } \\
\text { decision makers }\end{array}$ & $\begin{array}{l}\text { - Identify in-house equipment to be used or upgraded, types of decontamination supplies to store. } \\
\text { - Document HVAC and AHU systems in the facility. } \\
\text { - Select staging areas or warehouses for equipment and supplies. } \\
\text { - Determine likely decontamination method(s) for various scenarios. } \\
\text { - Determine barrier and isolation areas. } \\
\text { - Select and retain contractors for the decontamination team. } \\
\text { - Determine initial disposition of contaminated materials, staging and storage areas for waste. } \\
\text { - Initiate discussions with local waste-disposal facilities and wastewater management authorities, } \\
\text { including facility locations, capabilities, capacities, transportation plans, and costs. } \\
\text { - Discuss waste-disposal issues with state solid-waste-management authority. }\end{array}$ \\
\hline
\end{tabular}




\section{DECONTAMINATION}

\subsection{Summary}

Table 3-5. Summary of actions during the Decontamination Phase showing the approximate sequence of events.

\begin{tabular}{|c|c|}
\hline Responsible Personnel & Action \\
\hline $\begin{array}{l}\text { Planning Section: EU, with } \\
\text { input from TWG and ECC }\end{array}$ & $\begin{array}{l}\text { Develop cleanup goals and measurable cleanup performance criteria, as appropriate and } \\
\text { based on the results of characterization.- }\end{array}$ \\
\hline $\mathrm{UC}$ & Approve overall cleanup goals. \\
\hline $\begin{array}{l}\text { Planning Section: } \\
\text { Environmental Unit, with } \\
\text { input from TWG }\end{array}$ & $\begin{array}{l}\text { Develop the decontamination strategy, including assessment of potential environmental } \\
\text { impacts of decontamination. } \\
\text { Prepare the Remediation Action Plan (RAP), including: } \\
\text { - Areas to decontaminate and types of surfaces involved. } \\
\text { - Materials and structures to decontaminate in place or remove. } \\
\text { - What decontamination technologies to use (e.g., reagent and delivery system). } \\
\text { - Appropriate process parameters and analytical techniques. } \\
\text { - Placement of biological indicators, if necessary. } \\
\text { Include Ambient Air Monitoring Plan (AAMP) in RAP if fumigation is used. } \\
\text { Prepare Clearance Sampling and Analysis Plan (SAP), including: } \\
\text { - Clearance zones. } \\
\text { - Sampling approaches for each zone (targeted, biased, and random or statistical sampling). } \\
\text { - Aggressive air sampling, as necessary.- } \\
\text { - Prepare disposal plan, including estimated types and amounts of wastes; transportation } \\
\text { needs and costs; clearance sampling at disposal sites; and long-term monitoring, if } \\
\text { necessary }\end{array}$ \\
\hline $\begin{array}{l}\text { Operations Section: } \\
\text { Decontamination Group }\end{array}$ & $\begin{array}{l}\text { Perform source reduction. } \\
\text { Provide input to, and review and approve draft RAP and clearance SAP. }\end{array}$ \\
\hline $\mathrm{UC}$ & Approve the RAP and clearance SAP, with input from the ECC. \\
\hline EU & $\begin{array}{l}\text { Submit RAP and clearance SAP to USEPA to obtain a crisis exemption if using an } \\
\text { unregistered product for decontamination (sterilant or pesticide not EPA-approved). }\end{array}$ \\
\hline EU & $\begin{array}{l}\text { If fumigation is selected, develop an Emergency Response Plan to address potential } \\
\text { uncontrolled fumigation releases (e.g., from explosion, fire, or hurricane). }\end{array}$ \\
\hline $\begin{array}{l}\text { Operations Section: } \\
\text { Decontamination and } \\
\text { Sampling Groups }\end{array}$ & Perform all site preparations specified in the RAP. \\
\hline $\begin{array}{l}\text { Operations Section: } \\
\text { Decontamination Group }\end{array}$ & Conduct decontamination. \\
\hline $\begin{array}{l}\text { EU with input from } \\
\text { Decontamination Group and } \\
\text { TWG }\end{array}$ & $\begin{array}{l}\text { Evaluate whether decontamination process criteria are met; consult with ECC, on: } \\
\text { - Fumigation (temperature, relative humidity, fumigant concentration, and contact time as } \\
\text { well as biological indicators) } \\
\text { - Surface decontamination (e.g., limited surface sampling, contact time, pH). } \\
\text { Recommend additional decontamination activities, as necessary. }\end{array}$ \\
\hline Disposal Group & Develop disposal plan, and carry out disposal activities specified in the plan. \\
\hline
\end{tabular}




\section{DECONTAMINATION}

\subsection{Section 3 References}

AAMI (1995), Association for Advancement of Medical Instrumentation, Standards and Recommended Practices. Sterilization, Part I. Sterilization of Health Care Facilities; Sterilization, Part 2. Hospital Equipment and Industrial Process Control, Arlington, VA.

CDC (2005), Centers for Disease Control and Prevention, CDC Vessel Sanitation Program; available at www.cdc.gov/index.htm; click on Publications and Products.

CDC (2006), Centers for Disease Control and Prevention, Infection Control in Health-Care Facilities; available at www.cdc.gov/index.htm; click on Publications and Products.

Hawley, R. J. and J. P. Kozlovac (2004), "Decontamination,” in Biological Weapons Defense: Infections Disease and Counterbioterrorism (Humana Press, Totowa, NJ).

NRC (2005), National Research Council of the National Academies, Committee on Standards and Policies for Decontaminating Public Facilities Affected by Exposure to Harmful Biological Agents, Reopening Public Facilities After a Biological Attack (The National Academies Press, Washington, D.C.).

U.S. General Accounting Office (June, 2003), Capitol Hill Anthrax Incident, EPA's Cleanup Was Successful. Opportunities Exist to Enhance Oversight, GAO Publication GAO-03-686.

Weis, C. P., A. J. Intrepido, A. K. Miller, P. G. Cowin, M. A. Durno, J. S. Gebhardt, and R. Bull (2002), "Secondary Aerosolization of Viable Bacillus anthracis Spores in a Contaminated U.S. Senate Office," JAMA 288 (22), 2853. 


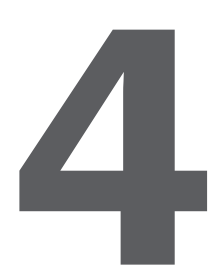

\section{CLEARANCE}

\section{Clearance}

The purpose of clearance is to decide whether or not to release an airport for reoccupancy (see Figure 1-1). The clearance process includes the following major components:

- Reviewing information from earlier phases, including characterization environmental sampling; source-reduction activities, if any; characterization data; and data from monitoring the decontamination process.

- Conducting clearance environmental surface sampling after decontamination and reviewing results.

- Performing aggressive air sampling for agents such as B. anthracis that can reaerosolize, and reviewing results from samples collected during aggressive air sampling.

All such information and data are reviewed relative to the clearance goal. The clearance goal should have been established before or while selecting the decontamination technique (see Section 3.2).

At most, but not all, sites with fumigations following the 2001 B. anthracis attacks, ECCs were established. The ECCs reviewed relevant cleanup data including adherence to key process variables and BI results from fumigations, as well as the nature, placement, and results of clearance environmental sampling. The ECCs then made recommendations on whether facilities were effectively decontaminated for re-use.

Although not mandated, it is recommended that the UC form an ECC to review and evaluate clearance data and recommend whether remediation is successful. ECC members should not be the same individuals as those who design the characterization and decontamination approaches. They should represent their own fields of expertise rather than the views of any agency and should be recognized by stakeholders and the public as subject matter experts (SMEs) in their respective fields. Members can include sterilization experts, sampling experts, toxicologists, industrial hygienists, epidemiologists, microbiologists, physicians and clinicians with expertise in infectious diseases and infection control, and experts from local departments of public health, with not more than one or two individuals drawn from any single field. The ECC is often chaired by a local public health official. The ECC should be created early and briefed on the proposed Sampling and Analysis Plan so that members are familiar with the sampling and analysis methods that will generate data for their review during the clearance process.

Resources necessary for clearance activities are the same as those identified in Table 2-1. Figure 4-1 shows the major activities associated with the Clearance Phase. 


\section{CLEARANCE}

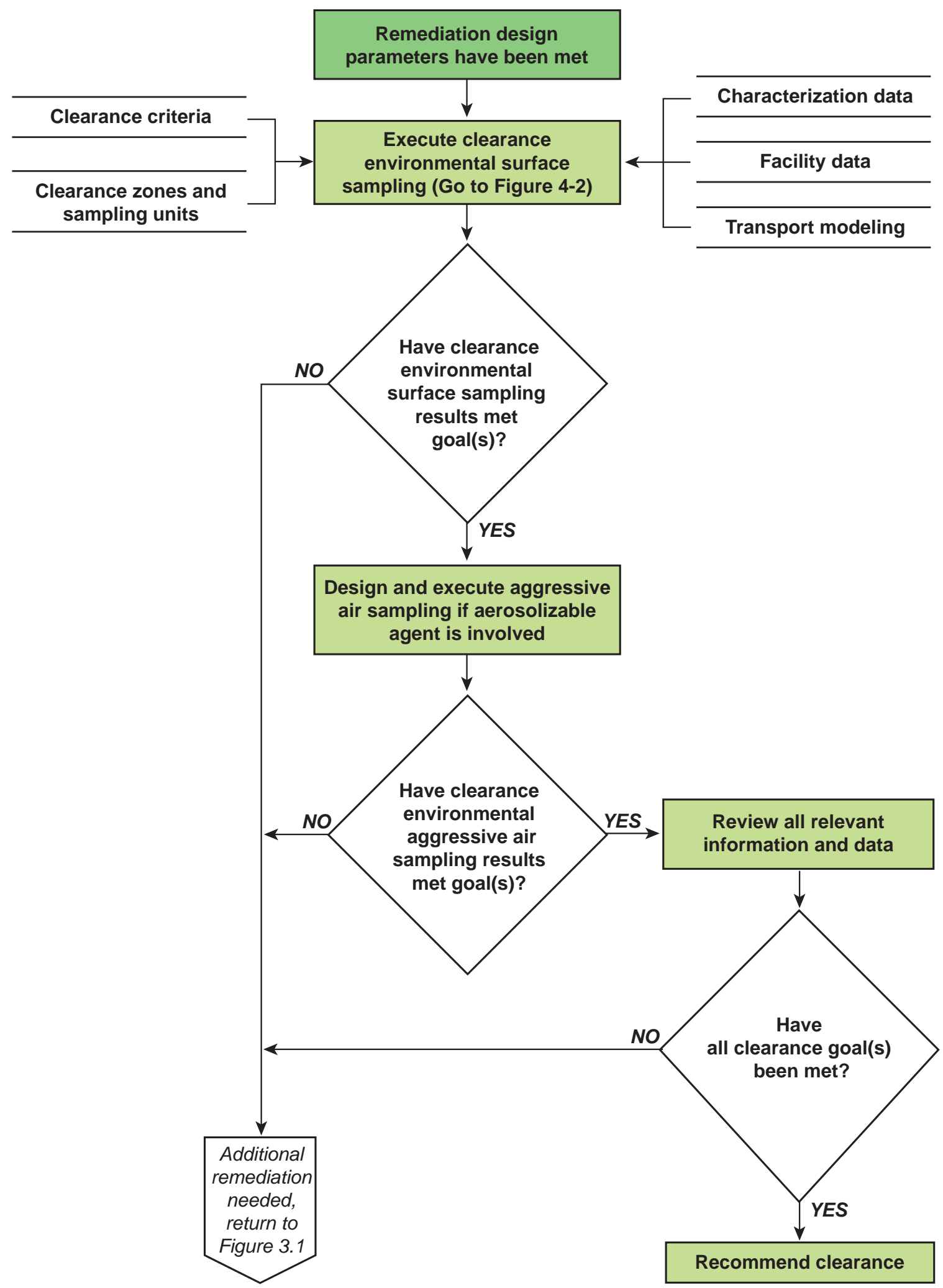

Figure 4-1. Major activities during the Clearance Phase. 


\section{CIEARANCE}

Clearance, like characterization, should be organized using a zone-by-zone approach. Zones should be defined in a flexible manner that makes sense to those performing the work. For example, if fumigation is performed, each fumigation zone is a natural clearance zone. Appendix D discusses zones in more detail; Appendixes $\mathrm{H}$ and $\mathrm{K}$ contain templates designed to help with the process. The remainder of Section 4 assumes that clearance assessment is organized using the same zones as those established for characterization, an approach that can be adopted regardless of the decontamination method. Such an approach helps relate clearance environmental sampling results with characterization sampling results. A positive clearance decision for the entire airport is made only after a positive clearance decision has been reached for every clearance zone.

\subsection{Review Clearance Goal}

Before selecting a decontamination technique, the overall cleanup goal (see Section 3.2) must be established along with an agreed-on process for judging whether the goal has been met. The goal depends on many factors, including — but not limited to - the type of contaminant released (agent and grade), location of contamination in the airport (e.g., at a ticket counter versus in an air cargo building), public perception of risk, scientific information on infectious dose and potential risk, and applicable environmental regulations.

Because of many uncertainties about such factors during 2001 anthrax events in the U.S., the goal that was used for judging the effectiveness of decontamination of contaminated sites was no growth of $B$. anthracis spores on any clearance environmental sample (either surface or air samples). It is the responsibility of the EU, with advice from the TWG, to review any standards in effect at the time of an event and decide what the goal should be. The ECC must concur with the clearance goal.

It is not certain at this time whether the same goal as that used for B. anthracis would be used in the event of contamination by some other BWA, or whether a measurable but sufficiently low level of some other BWA might be permitted after decontamination. In the latter case, the clearance goal would define how low is sufficiently low. The conservative assumption is that the goal used for $B$. anthracis cleanups in the past would be considered for another BWA, namely, no growth of viable agent on any clearance sample.

In Section 4, the terms "positive" and "positive result," when referring to a single sample, mean that growth of the agent was detected on the sample (i.e., the agent is infectious). The terms "negative" and "negative result" mean that growth was not detected on the sample.

\subsection{Plan for Clearance Environmental Sampling}

The clearance environmental sampling strategy is documented in the Clearance Sampling and Analysis Plan (SAP). It is essential to have a high level of confidence in a decision to reopen an airport. Unfortunately, it is not possible to prove by sampling that absolutely no viable or infectious agent is present after decontamination. Even if every square inch of a facility could be sampled, the following limitations apply:

- Sampling methods have less than $100 \%$ collection efficiency, meaning that the agent may be present in the environment, but not enough is collected in the sample to be detectable.

- Analytical methods have less than $100 \%$ extraction efficiency, meaning that the agent may be present in the sample, but the analytical method does not extract enough of it from the sample medium to be detectable. 


\section{CLIEARANCE}

- Even if both sampling and extraction had 100\% efficiency, analytical methods still have detection limits, meaning that the agent could be present in the environment but not be detectable by the analytical method.

- Some errors in collection, analysis, and documentation of samples, are inevitable.

Thus, even if all clearance sample results are negative, it is not certain that absolutely no viable (or infectious) agent remains in the environment. It is necessary to infer from a set of negative results that, at most, a negligible quantity of agent remains. That is, the locations or levels of remaining agent, if any, are sufficiently rare or sufficiently low that they present no unacceptable threat to human health. The important question that must be answered is how much sampling is necessary to make that inference with confidence. The EU should work with the ECC throughout development of the SAP to ensure that the ECC is satisfied that the plan is adequate.

In the B. anthracis decontaminations of 2001 and subsequently, three strategies were used for clearance surface sampling. The clearance goal for all three was no growth of B. anthracis on any sample. The three strategies are targeted, biased, and random sampling, as follows:

- Targeted sampling consists of "thoroughly sampling objects and/or areas where positive results for B. anthracis were previously found." See the Sterling mail facility SA-32 Environmental Clearance Sampling Plan (2003), in which this type of sampling was called "focused" sampling. Note that "targeted" sampling is now the preferred term, rather than focused sampling.

- Biased sampling preferentially targets "objects and/or areas that are adjacent to known contaminated locations, high-traffic areas, and objects and/or areas that are likely to experience high contact by personnel eventually reoccupying the facility." [See the SA-32 Environmental Clearance Sampling Plan (2003).] In an airport, the latter includes high-risk, high-use items, such as terminal counters, handrails, baggage-claim carousels, toilet facilities, and difficult-to-decontaminate areas, including air ducts and interior corners.

- Random sampling is "used to provide a certain amount of minimal coverage over areas not necessarily covered by biased or targeted sampling." [See the SA-32 Environmental Clearance Sampling Plan (2003).] In the B. anthracis cleanups of 2001 and thereafter, random sampling was used for areas where no B. anthracis contamination was found.

Targeted surface sampling is done at exactly the same locations where positive samples occurred during characterization. Biased surface sampling takes place in areas with some contamination that surround areas with heavy contamination. Grid/random sampling takes place in the remaining areas.

The choice of strategies needs to take into account how initial environmental sampling and characterization sampling were performed, where positive samples were found, the frequency and levels of contamination found, where no positives were found, and the types of samples obtained. See Appendix D for more information on clearance sampling strategies and related issues.

Multiple strategies can be used even in the same clearance zone. For example, targeted sampling would be used on surfaces in the immediate vicinity of an overt release location. Biased sampling would be used in nearby locations having high exposure potential. Random sampling would be used on large surfaces (floors, walls, and windows) at some distance from the release location. Inference from all three approaches is based on the idea that the number and placement of samples provides a high level of confidence that the agent, if present anywhere, will be detected somewhere. 


\section{CIEARANCE}

Inference from targeted sampling is also based on the following reasoning: If the responsible organization is confident that all areas of significant contamination were found during characterization, and the same areas are re-sampled during clearance and found to be clean, then there is confidence that decontamination was successful. This reasoning requires a belief that, because decontamination was successful in the known areas of significant contamination (i.e., areas selected for targeted sampling), it was also successful in areas with less contamination and areas of significant contamination that might have been missed during characterization. Inference from biased sampling is essentially the same.

Confidence that the agent will be detected at a particular location if it is present at that location depends on the sampling method. A sampling method with lower collection efficiency is less likely to detect the presence of the agent. Detection is more likely if the sampling method covers a large surface area (see Appendix C). When a statistical level of confidence is specified for random sampling, it is "statistically designed" as well as "random" sampling, and a required confidence level is set. The criterion is the same: all results must be negative. For example, the UC could require that there be a $95 \%$ confidence of detecting the presence of the agent if more than $5 \%$ of the surface area has the agent at detectable levels. This specification will determine how many samples must be collected. The U.S. Government Accountability Office (GAO) has suggested that statistically designed sampling could be used to increase confidence in the clearance decision when all results are negative (GAO 2005), and statistical sampling is included as an option in the interim-final National Response Team Technical Assistance for Anthrax Response (NRT 2005).

Random or statistically designed sampling makes the best sense for large surfaces where human contact occurs in a somewhat random or haphazard manner, such as floors, walls, and ceilings; in areas where relatively little is known about the contaminant distribution; or as a backup to targeted and biased sampling. The use of statistically designed sampling for other objects, such as ticket counters, drinking fountains, telephones, or escalators is more problematic because the objects are relatively few in number, and their complex physical structure makes representative sampling more difficult.

Statistical methods applicable to the environmental clearance decision can be found in many references, including, Gilbert (1987); Gilbert et al. (1996); Hardin and Gilbert (1993); Mulhausen and Damiano (1998); and USEPA (1996, 1997). Appendix D contains additional discussion and examples of ways in which random sampling can be statistically designed. Experts in the field of sampling and statistics should be consulted (and be a part of the TWG) if statistical sampling is used.

Figure 4-2 shows the process of developing a clearance environmental surface sampling plan. 


\section{CLEARANCE}

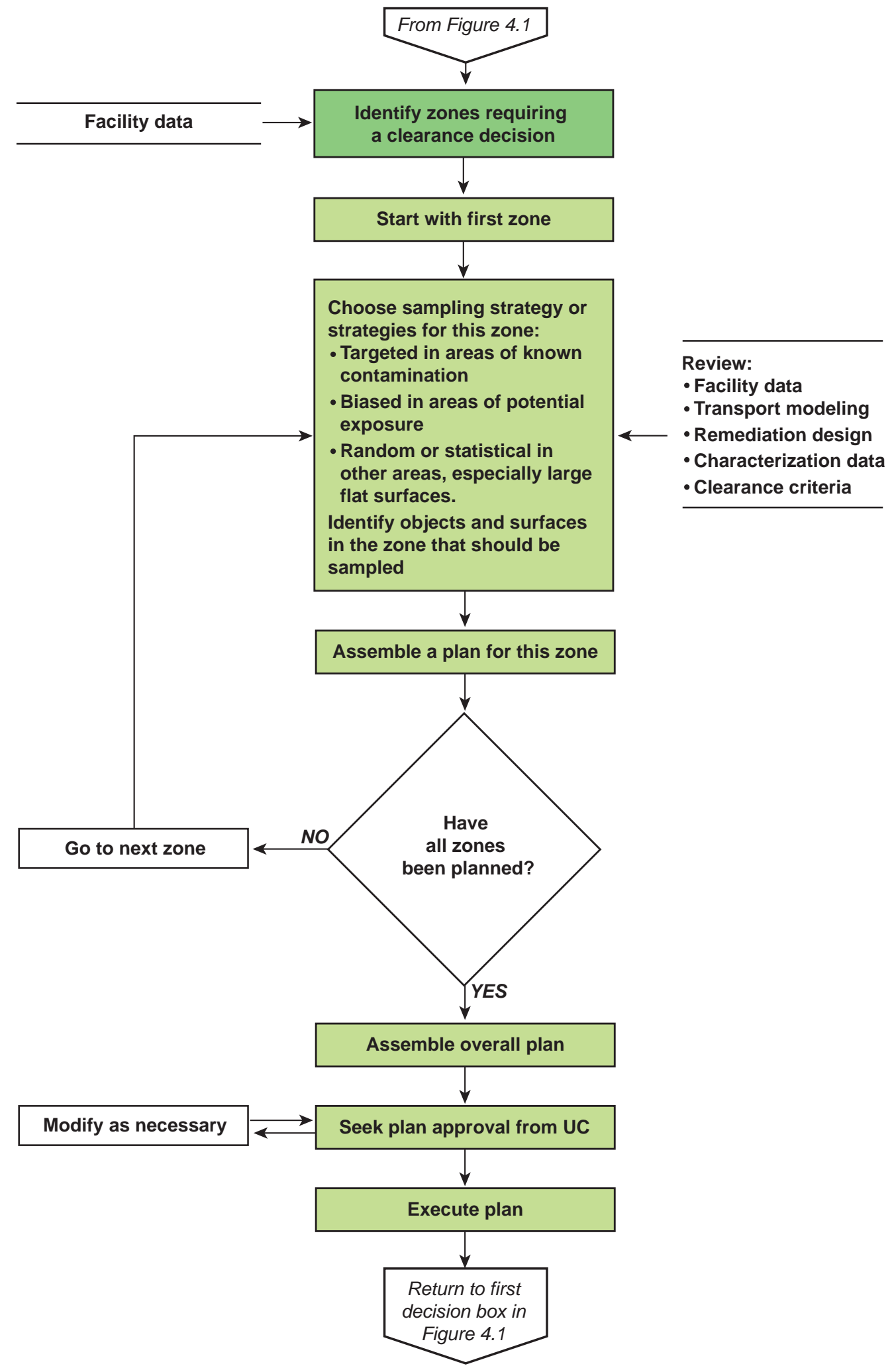

Figure 4-2. Developing a clearance environmental sampling plan (SAP). 


\section{CIEARANCE}

\subsubsection{Plan Aggressive Air Sampling}

In cleanups to date, the presence of detectable amounts of viable B. anthracis spores or other BWA after decontamination was unacceptable. To make such a determination, it is essential to sample in a manner that maximizes the likelihood of finding viable agent. Aggressive air sampling for aerosolizable agents is intended to do just this and is the last hurdle for clearance. The idea is to sample air during and after forced air is applied to exposed surfaces so that spores not detected during surface sampling are resuspended. Such air sampling takes place after all surface sampling is complete and after no growth of $B$. anthracis spores is demonstrated from those surface samples.

Fans are used to create turbulence, lifting particles such as B. anthracis spores from surfaces and into the air where air samplers can collect them. This method is described in the interim-final NRT Technical Assistance for Anthrax Response as follows: "While the area is under negative pressure, all surfaces are aggressively agitated and air is continuously disturbed while samples are collected. An air sampling method that maximizes the likelihood of detecting contamination should be used." (NRT 2005.) This method requires that areas be isolated and sealed, as they would be to prevent the release of fumigant. NIOSH has determined that a minimum of two room volumes of air should be collected in a given room to maximize the likelihood of capturing contamination in the samplers. In extremely large open areas, aggressive air sampling may not be feasible because it may not be possible to sample the amount of air (at least two room volumes) needed to produce meaningful results.

Air samplers are placed in a sampling zone. Sufficient samplers should be spaced together closely enough that particles are unlikely to be lifted and moved around without encountering a sampler. Samplers should be placed at various heights. Fans are placed in the zone and turned on immediately after the air samplers are turned on. In addition, sampling technicians (wearing appropriate PPE) carry hand-held fans and blow air across all accessible surfaces while the air samplers operate. This should be done in a planned and systematic manner, to ensure thorough coverage. Air samples are collected during the agitation. Workers in the area can wear personal sampling devices during the process.

After the process is complete, any suspended particles will re-settle in new locations. Therefore, comparisons of particulate concentrations at specific locations before (e.g., from characterization) and after aggressive air sampling are not meaningful. The expected effect of turbulence, if done thoroughly and for long enough, is to mix particles from all areas. The result is a physical averaging of particle concentrations (similar to compositing samples). Comparisons of averages before and after would be meaningful, but any comparisons involving variability — such as minimum, maximum, standard deviation, or range - are not.

Unlike the sampling units used for surface sampling (each unit represents a single type of surface or a set of similar objects), aggressive air sampling applies to an entire sampling zone. There is some ongoing debate over the pros and cons of operating NAUs during aggressive air sampling; however, NAUs were used in previous anthrax cleanups. This issue should be addressed on a case-by-case basis.

\subsection{Prepare and Execute Clearance Environmental Sampling Plan}

Using the concepts shown in Figures 4-1 and 4-2, the EU prepares a clearance environmental sampling plan (a part of the overall SAP), which is approved by the UC. Most environmental sampling plans, including post-decontamination clearance sampling plans, share certain basic elements. They include descriptions of the circumstances, statements of the authority under which the operation takes place, 


\section{CLIEARANCE}

summaries of applicable environmental laws and regulations, summaries of the kinds of decisions to be made, the rationale behind various decisions, technical information about the sampling and analysis methods, information about the kind of quality controls used, and types of PPE that the sampling teams use. Appendix $\mathrm{K}$ is a clearance plan template with sections for these kinds of topics.

Much of the necessary background information for the plan will be available from the Characterization Phase, including a set of sampling unit definitions (see Appendix H), estimates of locations of maximum contaminant levels, possibly a contaminant map, sample naming conventions, a database for analytical results, and other elements.

The clearance environmental sampling plan must be specific, including information about exactly where to take each sample or how to determine where to take each sample. The plan must include information on what sampling method(s) to use, how sampling locations will be determined in units where random sampling is used, how to package and transport each sample, how to document each sample, who instructs the necessary personnel, who collects the necessary supplies, and so on. It is important to ensure that the sampling staff can understand and follow the sampling plan exactly.

After the clearance environmental sampling plan is completed, the ECC, if one exists, may perform a conceptual review of the plan. Upon approval of the plan by the Operations Section Chief and the UC, clearance sampling commences.

\subsection{Clearance Decision}

The UC ultimately makes a clearance decision based on a judgment as to whether the criteria for decontamination verification and clearance have been met. The judgment considers the clearance goal, a thorough analysis of all sampling, process, and other data that are pertinent to the criteria for success, as outlined in the SAP and RAP.

After decontamination activities and clearance environmental sampling are completed, the EU, with input from the Decontamination Group and the TWG, reviews all pertinent data (e.g., fumigation parameters, characterization and clearance sampling data, and results from culture of BIs, if used). The EU determines whether clearance criteria are met and writes a report to the UC as to whether remediation has been successful and people may re-enter the site without using PPE. If the data indicate an unacceptable level of residual contamination, further decontamination and clearance sampling may be necessary. For additional certainty and credibility, the ECC provides a separate analysis of all pertinent data and makes an independent recommendation to the UC.

The UC reviews the clearance recommendations and confirms that facility, regulatory, and stakeholder needs are met such that the facility, or a part of it, can be cleared for re-occupancy. A similar process takes place for clearing decontaminated items for re-use. The Facility authority determines whether to reopen all or parts of the facility or whether to initiate restoration activities. 


\section{CLEARANCE}

\subsection{Pre-Incident Planning}

Table 4-1 identifies essential pre-incident planning activities related to clearance.

Table 4-1. Summary of clearance actions to be taken prior to a biological attack.

\begin{tabular}{|l|l|}
\hline \multicolumn{1}{|c|}{ Responsible Personnel } & \multicolumn{1}{c|}{ Pre-Incident Actions } \\
\hline $\begin{array}{l}\text { Airport authority decision } \\
\text { makers }\end{array}$ & $\begin{array}{l}\text { - Identify members of an Environmental Clearance Committee. ECC } \\
\text { members should review this Remediation Guidance document and } \\
\text { convene early, before characterization, if possible. }\end{array}$ \\
\hline
\end{tabular}

\subsection{Summary}

Table 4-2. Summary of actions during the Clearance Phase.

\begin{tabular}{|l|l|}
\hline \multicolumn{1}{|c|}{ Personnel } & \multicolumn{1}{c|}{ Action } \\
\hline $\begin{array}{l}\text { Planning Section: } \\
\text { Environmental Unit, with } \\
\text { input from TWG }\end{array}$ & Review and revise, as necessary, the incident-specific clearance SAP. \\
\hline UC with input from ECC & Approve the incident-specific clearance SAP if it was revised. \\
\hline $\begin{array}{l}\text { Operations Section: Sampling } \\
\text { Group }\end{array}$ & Perform clearance sampling. \\
\hline $\begin{array}{l}\text { Planning Section: } \\
\text { Environmental Unit with input } \\
\text { from Decontamination Group } \\
\text { and TWG }\end{array}$ & $\begin{array}{l}\text { Evaluate clearance SAP results. } \\
\text { Determine if cleanup criteria are met. } \\
\text { Recommend additional decontamination if necessary. } \\
\text { Write the final clearance report, and submit it to the UC. }\end{array}$ \\
\hline $\begin{array}{l}\text { EU and ECC, or ECC } \\
\text { independently }\end{array}$ & $\begin{array}{l}\text { Recommend whether facilities and items have been effectively } \\
\text { decontaminated. }\end{array}$ \\
\hline UC & $\begin{array}{l}\text { Conduct reviews and confirm that facility, regulatory, and stakeholder } \\
\text { needs are met. }\end{array}$ \\
\hline Facility authority & $\begin{array}{l}\text { Determine whether to reopen all or parts of the facility, or to initiate } \\
\text { restoration activities. If none of the above, further decontamination may } \\
\text { be warranted. }\end{array}$ \\
\hline
\end{tabular}




\section{CLIEARANCE}

\subsection{Section 4 References}

EPA (2002), U.S. Environmental Protection Agency, Guidance on Choosing a Sampling Design for Environmental Data Collection. U.S.EPA, EPA QA/G-5S (December 2002).

GAO (2005), U.S. Government Accountability Office, Anthrax Detection: Agencies Need to Validate Sampling Activities in Order to Increase Confidence in Negative Results, Testimony before the Chairman, Subcommittee on National Security, Emerging Threats, and International Relations, House Committee on Government Reform, House of Representatives, statement of Keith A. Rhodes, Chief Technologist, Center of Technology and Engineering, Applied Research and Methods, GAO-05-493T, April 2005.

Gilbert, R. O. (1987), Statistical Methods for Environmental Pollution Monitoring, (New York, NY, Van Nostrand Reinhold).

Gilbert, R. O., T. LeGor, et al. (1996), An Overview of Methods for Evaluating the Attainment of Cleanup Standards for Soils, Solid Media, and Groundwater, EPA Volumes 1, 2, and 3, Pacific Northwest National Laboratory, PNL-10908, EC-502.

Hardin, J. W., and R. O. Gilbert (1993). Comparing Statistical Tests for Detecting Soil Contamination Greater than Background (Pacific Northwest Laboratory, Richland, WA), PNL-8989, UC-630.

Mulhausen, J. R. and J. Damiano, (1998), A Strategy for Assessing and Managing Occupational Exposures (AIHA Press, American Industrial Hygiene Association, Fairfax, VA).

NRC (2005), National Research Council of the National Academies, Committee on Standards and Policies for Decontaminating Public Facilities Affected by Exposure to Harmful Biological Agents, Reopening Public Facilities After a Biological Attack (The National Academies Press, Washington, D.C.).

NRT (updated July, 2005), National Response Team, Technical Assistance for Anthrax Response, InterimFinal Draft; available only by electronic means (www.nrt.org).

Raber, E., A. Jin, K. Noonan, R. McGuire, and R. D. Kirvel (2001), “Decontamination Issues for Chemical and Biological Warfare Agents: How Clean Is Clean Enough?" International Journal of Environmental Health Research 11, 128-148.

Raber, E., T. Carlsen, K. Folks, R. Kirvel, J. Daniels and K. Bogen (2004), "How Clean Is Clean Enough? Recent Developments in Response to Threats Posed by Chemical and Biological Warfare Agents," International Journal of Environmental Health Research.

SA-32 Environmental Clearance Sampling Plan (2003), Attachment 10.9 to the report, Remedial Action Plan for Fumigation of SA-32 Diplomatic Pouch and Mail Facility, Sterling VA (proprietary; June 19, 2003).

USEPA (1996), U.S. Environmental Protection Agency, Geostatistical Sampling and Evaluation Guidance for Soils and Solid Media (review draft), U.S.EPA, Office of Solid Waste.

USEPA (1997), U.S. Environmental Protection Agency, Multi-Agency Radiation Survey and Site

Investigation Manual (MARSSIM), Washington, D.C., U.S.EPA, DOE, NRC, and DOD, NUREG-1575, EPA 402-R-97-016.

Zirschky, J. and R. O. Gilbert (1984), "Detecting hot spots at hazardous-waste sites.” Chemical Engineering 91, 97-100. 


\section{PRE-PLANNING
RECOMMNNATIONS}

\section{Recommendations for Pre-Planning}

As is emphasized throughout this document, many activities can greatly reduce the time required to restore airport operations if those activities are conducted before an actual BWA release. Recommended preplanning actions by airport officials are summarized here by topic. Table 5-1 summarizes the principal preplanning actions identified in all sections of this Remediation Guidance document organized by the phase of cleanup with which an item is most closely associated.

1. Develop a concept of operations (CONOPS) for cleanup of a BWA release specific to the airport. The CONOPS should show the structure of the organizations involved in cleanup and identify their specific roles and responsibilities. Formation of a TWG, an ECC, and selection of members are strongly recommended before an incident occurs. Issues pertaining to local, state, and Federal jurisdictions should be addressed, and stakeholders should be identified. The steps in the CONOPS will depend on the magnitude of a release, and may be dictated by the type of BWA used. The CONOPS should also identify the pros and cons of a Federal versus nonfederal response. The CONOPS can be maintained in a Data Supplement. Identify alternative, backup locations for the EOC.

2. Ensure all facility information is readily accessible. Locate all architectural drawings of terminals, boarding areas, and other areas. Locate all mechanical drawings of ventilation and drainage systems, and associated mechanical rooms. All potential entrance and exit points for gases, particles, or liquids should be identified (such as sumps, drain pipes, vent shafts, and the like). The information could be summarized in a Data Supplement for quick access and initial planning. It is essential that legible and intelligible facility information be immediately accessible to the remediation personnel. Consider placing the information on a geographical information system (with hardcopy backup) that would be controlled and maintained by airport personnel.

\section{Identify containment zones to prevent the spread of BWA and isolation zones to prevent the release} of fumigant. Assess the facility layout and identify potential sampling, characterization, fumigation, and decontamination zones. Identify logical containment and isolation zones, and stipulate the means by which the zones are to be established. Isolation can be established at connector halls between major terminal areas. Fire doors can assist in isolation. Life-safety zones are used for smoke control and are often serviced by dedicated air-handling units (AHUs). Because they are defined by the AHUs of the airport HVAC system, they constitute logical zones for characterization and decontamination. Decontamination zones are defined primarily by physical structures, such as fire doors or corridors that can be easily sealed in the event of a release.

4. Identify sampling and analytical resources. Determine who will collect samples, such as initial screening samples and subsequent characterization and clearance samples. Meet with the local LRN laboratory and discuss sample throughput, reporting of results, and surge capacity. If needed, line up additional LRN analytical laboratories that can be tapped in the event that many samples are to be collected.

5. Identify sampling zones and units. Identify logical sampling zones and sampling units for the airport. Decide how the airport can be logically subdivided to facilitate environmental sampling. Sampling zones may be similar to the containment and isolation zones, or they may be defined at a finer scale. It is possible that sampling zones and units may be different, depending on the agent released. However, it should be possible to construct sampling zones and units that could be reviewed and modified as necessary in the case of an actual event. 


\section{RECOPREEPLANNING}

6. Identify the most likely decontamination methods and experienced contractors to be used. Evaluate the strengths and weaknesses of available decontamination methods. Select the most appropriate methods to use for different BWA attack scenarios. In some cases, it may be possible to use or upgrade in-house decontamination equipment. Identify staging areas or warehouses for equipment and supplies. Decide on the types and amounts of decontamination supplies needed and whether to purchase them in advance (some materials may have a short shelf life). Select potential contractors to employ as members of the decontamination team.

7. Identify what to decontaminate in situ, remove for offsite treatment, or remove for disposal. In most cases, easily removed and replaced items should not be retained, whereas structural components will be decontaminated in place. The decontamination reagent used will affect the decision of what items may be left in place. Whereas treatment in place should reduce the costs of the source-reduction step, critical equipment and items should be identified for removal and treatment offsite. If existing decontamination methods are not compatible with certain equipment, then identify alternative, backup, or replacement equipment.

8. Determine disposition of contaminated materials, and identify staging and storage areas for waste. Decontaminating materials in place will reduce the potential for spreading contamination, but it may also damage certain equipment or materials. Disposition choices should be evaluated in advance of an event. Estimate waste-storage requirements on the basis of quantities of materials that might require disposal, and depending on the decontamination technologies of choice. Initiate discussions with local waste-disposal facilities, including municipal waste landfills; construction and demolition debris landfills; hazardous waste landfills; and hazardous, municipal, and medical waste incinerators, if available. Discuss waste-disposal issues with the State solid-waste-management authority. Discuss wastewater management issues (e.g., wastewater from chlorine dioxide scrubbers) with local wastewater treatment facilities. Identify necessary disposal permits, waivers, and exemptions; disposal facilities, capacities available, and transportation routes; and pre-arrange contracts if possible.

9. Write a new, generic Health and Safety Plan. Write a new HASP or re-evaluate an existing one on the basis of information in this Remediation Guidance document.

10. Identify backup facilities to continue commercial air service. In the event that one or more airport terminals is contaminated with a BWA, identify air cargo areas, hangers, and other infrastructure that might be used for the resumption of commercial air travel in some capacity.

11. Hold planning meetings at scheduled intervals. Airport personnel should meet with prospective UC and TWG members, responders, and stakeholders to continue to develop cleanup-related documents, policies, and guidance. Response plans will change over time as technologies advance and local, state, and Federal policies evolve.

12. Conduct training exercises. Airports should identify the scope of training activities appropriate for responding to BWA scenarios. Activities can range from simple, internal notification drills to full-scale, mass-decontamination exercises that take place over one or more days. 


\section{PRE-PLANNING RECOMMENDATIONS}

Table 5-1. Summary of principal actions to be taken by airport authority decision-makers prior to a biological attack. Actions are listed under the phase of cleanup with which they are most closely associated.

Initial Notification and Policy Actions

- Develop a seamless notification protocol (such as a phone tree with predetermined triggers and prepared text messages) for all responders and agencies (Federal, state, and local) tailored to each stage of a developing incident. (See Appendix A.)

- Develop a policy statement specifying the criteria for airport closure or suspension of operations after a BWA attack.

Concept of Operations Actions

- Identify members of a Unified Command, convene the UC, and review this Remediation Guidance document.

- Identify members of a TWG. Members are drawn from the CDC, USEPA, local public health, sampling contractors, and analytical laboratories. The TWG should review this Remediation Guidance document.

- Conduct training exercises with likely command personnel, including TWG members and other responder and agency representatives.

- Identify alternative locations for an EOC and ICP, preferably near the airport, but offsite in the event that an onsite EOC is contaminated with a biological agent.

Characterization-Related Actions

- Identify characterization and decontamination resources listed in Table 2-1.

- Identify potential sampling, characterization, fumigation, and surface-decontamination zones within airport buildings.

- Identify sampling units.

- Identify areas at the airport that can be used or cleared for staging and storing waste materials.

- Make accessible all facility architectural and mechanical drawings and update them as necessary.

- Periodically update HVAC blueprints and operating parameters.

- Periodically update building vulnerability assessments, and correct any deficiencies.

- Create a new or review an existing HASP.

Decontamination-Related Actions

- Identify in-house equipment that could be used or upgraded for decontamination activities.

- Select staging areas or warehouses for equipment and supplies.

- Determine likely decontamination method(s).

- Determine types of decontamination supplies to store.

- Select and retain contractors for the decontamination team.

- Determine initial disposition of contaminated materials.

- Identify staging and storage areas for waste.

- Initiate discussions with local waste-disposal facilities and wastewater treatment facilities.

- Discuss waste-disposal issues with State solid-waste-management authority.

Clearance-Related Actions

- Identify members of an Environmental Clearance Committee. ECC members should review this Remediation Guidance document and convene early, before characterization, if possible. 



\section{APPENDIXA}

\section{Considerations for the Notification Phase}

This appendix uses San Francisco International Airport (SFO) as the model airport for issues relevant to the Notification Phase. For more information on topics, such as the command structure created during the Notification Phase, refer to the National Response Framework (NRF; see DHS, 2008) and the National Incident Management System (NIMS, 2004). Persons working at SFO or other large airport security departments are likely to be trained in ICS, NRF, and NIMS.

The Notification Phase begins with recognition that an incident has occurred or is likely to occur. Identification of an incident involving a biological warfare agent (BWA) could be made on the basis of a detection system, such as BioWatch; an epidemiological alert; images from cameras or related surveillance equipment; intelligence information; phone calls or direct communication with SFO personnel; or data on casualties from police, the fire department, paramedics, hospitals, eye witnesses, or others.

Principal activities of the Notification Phase are:

- Receiving and assessing information.

- Identifying suspect release sites.

- Relaying key information to appropriate agencies.

Such activities do not necessarily occur in sequential order but may start at different times, run concurrently, or continue beyond the Notification Phase.

To evaluate incoming information and ensure that appropriate initial actions are taken, the SFO airport duty manager (ADM) or designee must notify appropriate internal and external response organizations and implement plans for the physical space, requisite communications systems, and personnel needed to staff an Incident Command Post (ICP). As part of the pre-planning effort, airport decision-makers should develop a seamless notification protocol (such as a phone tree with predetermined triggers and prepared text messages) for all responders and agencies (Federal, state, and local) tailored to the Notification Phase and to subsequent stages of a developing incident.

Other agency representatives that are updated on emerging details about a BWA incident would also notify appropriate agencies. Exactly what representative or organization would contact each agency would depend on the details of a specific BWA incident, such as who initially observed the suspicious behavior and what details precipitated a determination that a terrorist incident has taken place. Nevertheless, the primary result of initial notification of key agencies is creation of a command structure in the ICP.

The ICP could be operated independently by SFO personnel. However, it is more likely that the ICP would be operated with other local or Federal response agencies, such as the San Francisco Metropolitan Medical Task Force (SFMMTF) or the Federal Bureau of Investigation (FBI). The ICP would most likely be located at the SFO Emergency Operations Center (EOC) or at an alternative SFO EOC location. The first commander of the ICP would likely be the ranking San Francisco Fire Fighter because that individual would be on scene quickly and will be tending to any life-crisis issues. At some point within the first few hours after determining that a significant BWA event has occurred, the FBI will likely take control of the scene for intelligence and evidence gathering, but the FBI would probably not take command of the UC.

The following sections in Appendix A describe the roles of the ICP, witnesses, and others during the Notification Phase: 


\section{APPENDIXA}

- A.1 Process Incoming Reports.

- A.2 Differentiate between an Actual Attack and a Hoax.

- A.3 Notify Agencies.

- A.4 Take Initial Actions at the Scene.

Table A-1 summarizes the actions to be taken before and during the Notification Phase and the principal content of this appendix.

Table A-1. Summary of actions to be taken before and during the Notification Phase.

\begin{tabular}{|c|c|}
\hline Personnel & Action \\
\hline Airport personnel & $\begin{array}{l}\text { Complete the following pre-planning actions: } \\
\text { - Incorporate specific biological response plans into the facility's emergency } \\
\text { response plan, as appropriate. } \\
\text { - Develop a notification protocol for all facility personnel, responder, and agencies } \\
\text { (Federal, state, and local) tailored to each stage of a developing incident. }\end{array}$ \\
\hline $\begin{array}{l}\text { Facility emergency } \\
\text { management organization } \\
\text { and law enforcement } \\
\text { or other response } \\
\text { organizations }\end{array}$ & $\begin{array}{l}\text { Facility receives notification that: } \\
\text { - A biological incident is detected or suspected, or } \\
\text { - Information about a developing threat is received from an agency or responsible } \\
\text { person as the result of a detection system (e.g., BioWatch), medical surveillance, } \\
\text { or epidemiologic investigation. }\end{array}$ \\
\hline $\begin{array}{l}\text { Airport personnel or } \\
\text { witnesses present at the } \\
\text { scene }\end{array}$ & $\begin{array}{l}\text { - Protect self, and move away from the source of exposure. } \\
\text { - Dial } 911 \text { when it is safe to do so. } \\
\text { - Inform first responders of all information known. }\end{array}$ \\
\hline $\begin{array}{l}\text { Incident Command Post } \\
\text { personnel }\end{array}$ & $\begin{array}{l}\text { - } \text { Gather all pertinent information from verbal reports. } \\
\text { - } \quad \text { Evaluate all reports, data, and intelligence information. } \\
\text { Follow previously established notification protocol to: } \\
\text { - Notify all relevant agencies of an actual event or threat. } \\
\text { - Inform first responders of all essential information. } \\
\text { - Disseminate information, including preliminary risk communication and public } \\
\text { - } \quad \text { Nealth directives. }\end{array}$ \\
\hline
\end{tabular}




\section{APPENDIXA}

\section{A.1 Process Incoming Reports}

This document assumes that a BWA incident is identified from a epidemiological alert or through surveillance or intelligence information. However, it is possible that initial incoming information may take the form of verbal reports. First reports might involve direct communication with SFO personnel, eyewitnesses, or others. To the extent possible, the following details should be recorded from incoming reports:

- Name and telephone number of the reporting individual.

- Title of reporting individual (e.g., physician, FBI agent, or citizen witness).

- Date and time of verbal report.

- Time and location of the incident (for example, place within an airport terminal or nearest gate within a boarding area).

- Visual observations concerning the release, signs and symptoms of any victims, or other pertinent information.

- Extent of injuries and number of victims.

- Demeanor of victims (for example, panicked).

- Nature of any immediate actions taken.

\section{A.2 Differentiate between an Actual Attack and a Hoax}

In verifying that a threat is credible or that an incident is real, decision-makers at the ICP, in consultation with other appropriate organizations, need to consider several possibilities at the outset. The possibility of a hoax must be evaluated in the absence of physical data or actual casualties. If the only information available is a letter or phone call from a party claiming responsibility, and casualties are zero, then the incident may be a hoax. Inappropriate handling of a hoax could result in indirect casualties arising from panic. The credibility of reporting individuals or other data is key in evaluating the possibility of a hoax. It is important to recognize that the latency of reported casualties can differ depending on the BWA(s) used during an attack. Biological agents can cause delayed health effects, whereas chemical agents and biotoxins cause more immediate health effects. To address the issue of whether an event is an actual attack or a hoax, the ICP will need input from several entities including the FBI, Transportation Security Administration (TSA), Centers for Disease Control and Prevention (CDC), local public health, and the Laboratory Response Network (LRN). Such agencies will be updated by the ICP as information about the incident matures. The incident may proceed in stages, from a suspicious incident to a presumptive positive event to a confirmed attack.

\section{A.3 Notify Agencies}

If the IC or UC is told or concludes that an incident is not a hoax, notification of community officials, regulatory personnel, first responders, and facility cleanup organizations in a timely manner is vital during the Notification Phase. Other notification chains are activated as a function of other criteria. For example, if polymerase chain reaction (PCR) results are positive from a collected sample, the LRN or state department of health services initially notifies the CDC Director's Emergency Operations Center, local FBI WMD coordinator, and local law enforcement authorities. When a BWA release or threat of a release from a terrorist incident could threaten human health and safety or the environment, the agencies identified in Table A-2 must be verbally notified. Some agencies will receive duplicative notifications. 


\section{APPENDIXA}

Table A-2. Agencies to be notified if a BWA release threatens health, safety, or the environment.

\begin{tabular}{|l|}
\hline \multicolumn{1}{|c|}{ Agency to Notify } \\
\hline National Response Center \\
\hline Federal Emergency Management Agency (FEMA) \\
\hline $\begin{array}{l}\text { Environmental Protection Agency (EPA) Federal On Scene Coordinator (through the } \\
\text { National Response Center) }\end{array}$ \\
\hline Screening Control Center (Transportation Security Administration) \\
\hline TSA Deputy Manager \\
\hline Transportation Security Operations Center (TSOC) \\
\hline San Francisco Police Department, Airport Bureau \\
\hline Federal Bureau of Investigation \\
\hline Joint Terrorism Task Force (JTTF) (San Francisco FBI Field Office) \\
\hline San Francisco Fire Department \\
\hline San Francisco Metropolitan Medical Task Force \\
\hline Public Health Officer (CDC at SFO) \\
\hline San Mateo Public Health Officer \\
\hline BART Operations Control Center \\
\hline California Office of Emergency Services (OES) \\
\hline Environmental Regulatory Agencies (CalEPA coordinates) \\
\hline
\end{tabular}

Essential information must be provided to responders to ensure their safety. If a release is intentional, the Joint Terrorism Task Force (JTTF) is alerted by the FBI special agent who responds to the incident, which will bring additional emergency resources to the scene. Suspicious activity, terrorist threats, and actual incidents with a potential or actual terrorist nexus are reported immediately to a local or regional JTTF. Subsequently, the FBI Strategic Information and Operations Center (SIOC) immediately reports the terrorist threat (if the FBI deems the threat to be credible) or the actual incident to the National Operations Center (NOC) and to the National Counterterrorism Center (NCTC). The NOC serves as a national-level, multiagency, situational awareness and operational coordination center. In addition, actual incidents, regardless of whether or not there is a terrorist nexus, are reported immediately to the NOC by appropriate governmental and nongovernmental entities.

Beyond the ICP, local and state Emergency Operations Centers would be created or activated, if necessary, in addition to the Joint Field Office (JFO) that coordinates Federal assistance and supports incident management activities. The JFO communicates directly with the NOC. A Principal Federal Official (PFO) would head the JFO. However, depending on the magnitude of the disaster, a PFO may not be designated. In that case, the Federal Coordinating Officer provides the Federal lead. 


\section{APPENDIXA}

\section{A.4 Take Initial Actions at the Scene}

Airport personnel who witness a BWA release, or individuals who exhibit signs or symptoms of exposure, should:

- Protect themselves by moving a safe distance away (upwind) from a suspect source.

- Dial 911 when it is safe to do so.

- Inform first responders of all information known about the incident.

\section{A.5 References}

DHS (March 2008), Department of Homeland Security, National Response Framework; available at http://www.fema.gov/emergency/nrf/.

NIMS (March 1, 2004), National Incident Management System, document available from FEMA at 1-800-480-2520, Option 4, ask for FEMA 501. 



\section{APPENDIX B}

\section{Considerations for the First-Response Phase}

This appendix uses San Francisco International Airport (SFO) as the model airport for issues relevant to the First-Response Phase. For more information on topics related to first response, refer to the National Response Framework (NRF; see DHS, 2008) and the National Incident Management System (NIMS, 2004). Persons working at SFO or other large airport security departments are likely to be trained in ICS, NRF, and NIMS.

During the initial phases of response to a bioterrorism incident, emergency activities are centered on saving human life. The First-Response Phase consists of initial actions on the part of airport personnel and San Francisco Police Department, Airport Bureau, officers who are present at the scene of a biological warfare agent (BWA) incident, along with additional outside first responders, such as the San Francisco Fire Department and San Francisco Metropolitan Medical Task Force (SFMMTF) who are called upon to stabilize the emergency. The First-Response Phase may include hazardous material (HAZMAT) and emergency actions, forensic investigation, public health actions, and screening sampling.

Immediately following a terrorist incident, airport personnel who are at the scene may need to function without outside resources for a critical time. Such personnel can perform several tasks if they are properly trained and instructed. Examples include organizing an evacuation of a terminal or airport, shutting down operations, attending to casualties, and supporting medical response teams.

The following sections describe considerations for the First-Response Phase:

- B.1 Personnel Training Prior to an Emergency.

- B.2 Airport First-Response Actions.

- B.3 Nonairport First-Responder Actions.

Table B-1 summarizes actions to be taken before and during the First Response Phase. 


\section{APPENDIX B}

Table B-1. Summary of actions to take before and during the First-Response Phase.

\begin{tabular}{|c|c|}
\hline Personnel & Action \\
\hline Airport personnel & $\begin{array}{l}\text { Complete the following pre-planning actions: } \\
\text { - Develop a policy specifying criteria for closure or suspension of operations after } \\
\text { a bioattack. The policy should incorporate public health strategies and address } \\
\text { management of employees, rolling stock, and patrons. } \\
\text { - Identify prospective members of a Unified Command (UC); convene the UC, and } \\
\text { review the facility's Remediation Action Plan. } \\
\text { - Conduct training exercises with likely command personnel, including responder and } \\
\text { agency representatives. }\end{array}$ \\
\hline $\begin{array}{l}\text { Airport personnel initially } \\
\text { present at the scene }\end{array}$ & $\begin{array}{l}\text { - Maintain a safe distance from the source of exposure. } \\
\text { - Assist in maintaining the safety of other responders. } \\
\text { - } \quad \text { Don personal protective equipment before attempting rescue actions. } \\
\text { - } \\
\text { and peport any symptoms (e.g., possibly associated with biological toxins) to responders } \\
\text { Establish hot, warm, cold zones; secure the scene to preserve evidence. }\end{array}$ \\
\hline $\begin{array}{l}\text { Incident Commander or Facility } \\
\text { Manager at the ICP }\end{array}$ & $\begin{array}{l}\text { Activate the following, or coordinate with law enforcement and emergency operations } \\
\text { personnel, as needed: } \\
\text { - Security personnel. } \\
\text { - } \quad \text { FBI and/or local law enforcement (will likely control the crime scene). } \\
\text { - } \quad \text { Fire department personnel. } \\
\text { - } \quad \text { Public health and medical personnel. } \\
\text { - } \quad \text { HAZMAT and/or other screening sampling teams. } \\
\text { - } \quad \text { Local USEPA On Scene Coordinator. }\end{array}$ \\
\hline $\begin{array}{l}\text { Other first responders called on } \\
\text { to assist }\end{array}$ & $\begin{array}{l}\text { - } \\
\text { - Evaluate HAZMAT actions needed to stabilize the situation. } \\
\text { - } \quad \text { Identify and prioritize contaminated areas and operations. } \\
\text { - Identify the agent used during the attack [i.e., if field-portable, real-time polymerase } \\
\text { chain reaction (PCR) units are available, such as RAPID or the Cepheid Smart Cycler]. } \\
\text { - Determine whether the threat continues. }\end{array}$ \\
\hline $\begin{array}{l}\text { Incident Commander or Facility } \\
\text { Manager at the ICP }\end{array}$ & $\begin{array}{l}\text { - Continue to inform responders and agencies about developing incident details; continue } \\
\text { risk communication. } \\
\text { - Control access and/or isolate the affected area, and contain contamination. } \\
\text { - Transition to a UC as additional agencies respond. } \\
\text { - Plan and conduct initial screening sampling for analysis of bioagent. }\end{array}$ \\
\hline CDC and local public health & $\begin{array}{l}\text { - Perform laboratory analysis of environmental screening samples. } \\
\text { - If laboratory analysis yields a confirmed positive result, determine if there is a } \\
\text { significant risk to public health. } \\
\text { - Gather new information, as necessary, to determine risk to public health (e.g., from } \\
\text { agent viability results, medical epidemiological surveillance, additional environmental } \\
\text { sample analysis, and available intelligence). }\end{array}$ \\
\hline IC or UC & Determine if facility operations should be sustained, diverted, or suspended. \\
\hline
\end{tabular}




\section{B.1 Personnel Training Prior to an Emergency}

Designated response personnel who are normally resident in the airport during working hours should be trained at the Occupational Safety and Health Administration (OSHA) operations level [29 CFR 1910.120 (e) and (p)], including 8 hours of training plus "awareness level" competency. Responders at the OSHA operations level are those individuals “... who respond to releases or potential releases of hazardous substances as part of the initial response to the incident for the purposes of protecting nearby persons, property, or the environment from the effects of the release. Operations level personnel are trained to respond in a defensive mode without actually attempting to stop the release." Many portions of a HAZMAT response are similar to the response to a BWA incident. The principal differences are that airport responders may encounter mass disruption, face dispersing biological agents, or need to secure a crime scene.

Custodial, security, maintenance, and other airport staff routinely present at the airport as part of their job functions should have training in cardiopulmonary resuscitation (CPR) and first aid. Such personnel are not expected to perform decontamination functions, but basic training in the medical aspects of biological agents could potentially reduce casualties and fatalities.

A select group of airport staff resident in the facilities could be trained to organize an immediate casualty medical-support area following a BWA attack. Air-traffic controllers should have training in several areas relevant to a BWA incident: deciding whether or not to stop airplanes from departing or arriving, initiating emergency procedures to control and evacuate passengers and visitors, identifying emergency routes, communicating with the Incident Command Post, and performing related functions.

\section{B.2 Airport First-Response Actions}

Airport first responders should:

- Maintain a safe distance from the source of a BWA and initially move or remain upwind.

- Assist in maintaining the safety of other responders.

- Wear the proper personal protective equipment (PPE) before attempting any rescue actions, including emergency decontamination of victims to save lives. The appropriate level of PPE should be identified during pre-planning activities.

- Note any symptoms exhibited by victims, which could assist in identifying a biological toxin in the absence of other detection and identification mechanisms.

- Report symptoms observed to appropriate responders and the Incident Command Post.

- Assist in establishing hot, warm and cold zones. The hot zone is an area with actual or potential contamination and the highest potential for exposure to the contaminant; it is equivalent to the red zone, exclusion zone, or restricted zone. The warm zone is transition area between hot and support zones, where responders enter and exit the exclusion zone. The cold zone is the area that is free from contamination and that may be safely used as a planning and staging area. Cordon off the area once all living victims have been removed, and secure the scene to preserve evidence.

Airport managers should work with first responders to develop plans for identifying as many potentially exposed people as possible before they leave the airport. A list of such people would be of considerable help in follow-up efforts to vaccinate exposed individuals or to inform them of other medical interventions 


\section{APPENDIX B}

or surveillance requirements if the pathogen proves to warrant such measures. Preprinted handouts and information packets could be prepared in advance for such purposes.

\section{B.2.1 Calculate Time Since Exposure}

Although the time since exposure to a BWA is not required to develop a decontamination plan, it can provide important information for emergency-response personnel who are treating victims. In some cases, the time since exposure will be the same as the time since release. However, the two times will differ under other circumstances. If available, remote camera video images should be reviewed to help establish the time since exposure, and the information should be passed along to personnel who are treating victims.

\section{B.2.2 Coordinate with Nonairport First Responders}

Agencies that contribute non-airport first responders include the fire department, police department, Federal Bureau of Investigation (FBI), Centers for Disease Control and Prevention (CDC), and others who may be called to the scene.

\section{B.3 Nonairport First-Responder Actions}

When non-airport first responders, such as personnel from the fire department and the Environmental Protection Agency (EPA) arrive, they will work with airport personnel to stabilize the emergency and identify the BWA agent. HAZMAT and emergency actions take place when first responders arrive on the scene and establish control of the situation. They set up an Incident Command Post, initiate any needed rescue operations, mitigate any life-threatening or hazardous conditions (e.g., fire or explosion), and conduct preliminary tests to determine whether the threat substance is organic or likely to be a hazard. They also contact law enforcement and other personnel, as needed.

\section{B.3.1 Stabilize the Emergency}

Depending on whether the agent used during an attack is known or unknown (that is, not yet identified), different actions may be required on the part of first responders who are called in to stabilize the emergency. If the agent is not known, first responders must evaluate whether emergency HAZMAT actions are needed to stabilize the immediate situation. The term HAZMAT is used in this context because biological agents or other materials that could potentially be used in a terrorist attack can be hazardous to health and are treated as hazardous materials by first responders. Emergency actions for BWA incidents can include, but are not limited to:

- Immediate decontamination with soap and water.

- Evacuation of the site.

- Shutdown of affected operations.

- Additional notification of workers or agencies.

- Use of containment procedures to minimize spread of the agent.

If emergency HAZMAT actions are necessary, responders need to identify and prioritize the contaminated areas and operations. If emergency HAZMAT actions are not needed, then the immediate emergency is stabilized to the extent possible, and responders must take initial steps toward identifying the agent before appropriate next steps can be determined. 
It is important to realize that a BWA incident may not be detected until several days or weeks after an actual attack. However, once an event is recognized, the situation needs to be evaluated at that time as an emergency until the emergency is stabilized.

\section{B.3.2 Screening Environmental Sampling}

Screening sampling is likely to commence during the First-Response Phase to obtain preliminary information on the presence of a BWA. Initial sampling may also be conducted to begin developing information on agent type, concentration, and viability. Sampling activities continue in more depth during the subsequent Characterization Phase.

A series of questions must be addressed by appropriate experts [e.g., from the FBI, CDC, or Laboratory Response Network (LRN) analytical laboratory] to determine whether an agent is an organism (viable, persistent, or infectious) or whether the agent is a chemical or toxin and is present in sufficient concentration to cause injury or disease. For example, if the agent is an organism, but the organism is not viable, then no immediate threat exists, re-entry may be allowed, and operations can resume. However, the crime scene must be preserved for subsequent forensic investigation. If preliminary tests indicate the likely presence of a hazardous biological substance, the FBI will likely commence a forensic investigation to identify the agent and determine its specific genetic, physical, and chemical properties; search for other types of evidence; establish a possible source of the contamination; and determine the responsible party. Initial samples are sent to a LRN laboratory for analysis and to confirm the identity and viability of the contaminant.

It may take several days to obtain definitive answers, especially ascertaining whether a BWA is viable or not. Moreover, the minimal infectious dose for many biological agents is not well established. In such cases, a judgment must be made about whether to proceed with emergency response and subsequent actions as though the agent continues to pose a threat. If the agent is not an organism, then it is by default a chemical, biological toxin, or radioactive material, and its identity and concentration are key factors. If any identified agent is present in sufficient concentration to cause injury or disease, then agent-specific information is used as a basis for performing initial or continuing HAZMAT and emergency response actions. Contaminated areas and operations must be identified and prioritized. Attention then shifts to site-specific conditions for characterization and risk assessment activities.

\section{B.3.3 Public Health Actions}

If the LRN analytical results confirm the presence of a BWA, the responsible public health agency involved in the response will commence appropriate public health actions, such as treatment and decontamination of potentially contaminated individuals, distribution of prophylaxis, and medical examinations. The primary public health agency for SFO is the San Mateo County Department of Public Health.

A Joint Information Center (JIC) should be established immediately to coordinate all public affairs activities and media releases regarding a BWA incident. An Information Officer (IO) who reports to the Incident Command or Unified Command should be appointed to develop and release information about the incident to news media and to all agencies and organizations involved. Targeted communication must evolve in synchrony with the phases of response and must be directed toward phase-specific activities. The JIC staff should be familiar with the basic tenets of emergency risk communication and with the unique information requirements of each phase. The operational requirements of each phase will vary according to the nature and longevity of a crisis. 


\section{APPENDIX B}

\section{B.4 References}

DHS (March 2008), Department of Homeland Security, National Response Framework; available at http://www.fema.gov/emergency/nrf/.

NIMS (March 1, 2004), National Incident Management System, document available from FEMA at 1-800-480-2520, Option 4, ask for FEMA 501. 


\section{APPENDIXC}

\section{Available Biological Sampling and Analysis Methods}

This appendix presents accepted sampling procedures used to determine the presence of Bacillus anthracis (B. anthracis) spores in large buildings (Sanderson et al. 2002). Future methods may be more efficacious; updates to this sampling protocol should occur as improved methods are developed. Early coordination with all laboratories that will be used for sample analysis is essential to determine capabilities and the analytical processes they use. Such coordination ensures that samples will be taken and prepared in a way acceptable to the laboratories.

Sample collection methods can be grouped into three broad types: bulk, surface, and air sample collection. Each sample-collection type has specific advantages in particular applications, but because of the complex nature of transportation facilities, such as airports, all method types may be needed to adequately characterize site contamination.

The quantity and type of background microorganisms in environmental samples can affect detection sensitivity. For this reason, characterization and clearance sampling should be the same so that results are comparable.

\section{C.1 Sample Collection Methods}

The sample collection methods described in this appendix are the current methods recommended by the Centers for Disease Control and Prevention (CDC). However, method collection efficiencies and limits of detection have not been established; thus, analytical results should be evaluated and acted upon with the knowledge that the reported values may not represent the actual, viable B. anthracis surface count.

\section{C.1.1 Bulk Sample Collection}

Bulk samples are high-volume samples collected to characterize biological agent contamination in relatively large-volume materials, such as surface dust accumulations and heating, ventilation, and air conditioning (HVAC) filters. To collect bulk samples:

1. Scoop or spoon the sample into sterile bags, then seal and double-bag to ensure that the sample is not affected by the cleaning described in the next step. Seal the double bag.

2. After sealing, clean the outside of the bag with a 0.5- to 0.6-percent sodium hypochlorite solution. Typical household bleach sold in the United States contains approximately 5 to 6 percent sodium hypochlorite.

3. Place the sealed sample bag inside another unused, self-sealing bag, and ship according to applicable CDC guidelines and regulations. The following information, as well as an appropriate chain-ofcustody, is necessary for the acceptance of samples by the laboratory: discrete sampler identifier; sample location; size of the sampled area; sample type, time, and date that the sample was taken; and name and initials of the person collecting the sample (CDC 2002).

4. Take appropriate precautions to prevent secondary spread of spores from bulk samples. Such samples pose exposure concerns for laboratory personnel and should only be sent to a laboratory operating at biosafety level 3 (BSL-3). 


\section{APPENDIXC}

\section{C.1.2 Surface Sample Collection}

Surface samples are collected to characterize biological agent contamination resulting from deposition of aerosols on indoor vertical and horizontal surfaces. Depositional surfaces found in a typical transportation facility include both nonporous and porous surfaces. Nonporous surface samples are typically collected by moistened wipes or swabs, by direct contact with growth medium, or by adhesive strips. Porous surface samples are generally collected by vacuuming. Extraction methods vary widely and are dependent on collection.

\section{C.1.3 Wipe and Swab Sample Collection Methods}

Several absorptive media are available for wipe or swab sample collection on nonporous surfaces or objects. In the past, sterile cheesecloth or cotton wool-tipped sticks were recommended for wipe and swab media, respectively. Then noncotton wipes or swabs, such as rayon and polyester, became preferred because of updated laboratory analytical procedures. An even more recent report from the CDC indicates that moistened cotton and macrofoam swabs are more efficient at sampling B. anthracis spores than moistened rayon or polyester swabs (Rose et al. 2004). Check with the CDC and analytical laboratory to determine the best and most current collection media because those resources evaluate old and new products. The collection media must be sterile and used with a sterile wetting agent, such as water, a saline solution, or a phosphate-buffered solution. Hung, Miller, and Dillon (2005) recommend sterile 0.1-percent peptone with 0.01-percent Tween ${ }^{\circledR} 80$ as the wetting agent.

\section{C.1.3.1 Wipe Sample Collection}

Wipe sample collection is generally used for larger, nonporous surface areas, such as ventilation ductwork, table and counter tops, file cabinets, and noncarpeted floors. To collect a wipe sample:

1. Wear sterile, nonpowdered examination gloves over the gloves that are part of the standard PPE, and change gloves between samples.

2. Moisten a small synthetic (rayon or polyester) sterile gauze pad with sterile water, saline, or phosphate-buffered solution using aseptic technique to prevent cross-contamination.

3. Wipe a known surface area using a sterile template with an area greater than 100 square centimeters. Wipe thoroughly using several horizontal strokes, fold the exposed side of the pad and make several vertical strokes over the surface area.

4. After sample collection, place the gauze wipe in a pre-labeled, sterile. 50-ml Blue Falcon screw-top tube (Becton Dickinson Labware, Franklin Lakes, NJ), and sealed with a cap.

5. After sealing, place in a sterile bag, seal it, and clean the outside of the bag with a 0.5- to 0.6-percent sodium hypochlorite solution.

6. Place the sealed sample inside another unused, self-sealing bag, and ship according to applicable CDC guidelines and regulations. The following information, as well as an appropriate chain-ofcustody, is necessary for the acceptance of samples by the laboratory: discrete sampler identifier, sample location, size of the sampled area, sample type, time and date that the sample was taken, and name and initials of the person collecting the sample (CDC 2002). 


\section{APPENDIXC}

Current surface sample methods for microbial contaminants are designed to sample small areas. Recent studies, however, show that sampling larger areas $\left(1 \mathrm{~m}^{2}\right)$ results in greater sensitivity and requires the collection of fewer samples (Buttner et al. 2004a).

\section{C.1.3.2 Swab Sample Collection}

Swab sample collection is best used for small, smooth nonporous surfaces or objects that do not have a large accumulation of dust or are difficult to sample, such as, computer keyboards, electronic equipment, machinery, and ventilation grills. To collect a swab sample:

1. Moisten a sterile rayon or polyester swab with sterile water, saline, or phosphate-buffered saline solution using aseptic technique to prevent cross-contamination.

2. Wipe a known surface area using a sterile template with an area less than 100 square centimeters. Swab samples are collected by swabbing the selected surface by moving the swab back and forth across the surface with several horizontal strokes, then several vertical strokes. The swab is also rotated during sampling to ensure that the entire surface of the swab is exposed.

3. After sample collection, the swab is placed in a pre-labeled, sterile 50-ml Blue Falcon screw-top tube (Becton Dickinson Labware, Franklin Lakes, NJ) and sealed with a cap.

4. After sealing, place in a sterile bag, seal it, and clean the outside of the bag with a 0.5- to 0.6-percent sodium hypochlorite solution.

5. Place the sealed sample inside an unused, self-sealing bag, and ship according to applicable CDC guidelines and regulations. The following information, as well as an appropriate chain-of-custody, is necessary for the acceptance of samples by the laboratory: discrete sampler identifier, sample location, size of the sampled area, sample type, time and date that the sample was taken, and name and initials of the person collecting the sample (CDC 2002).

\section{C.1.4 Vacuum Sample Collection}

Sample collection from large porous and nonporous surfaces, such as, carpeting, wallboard, painted walls, ventilation system filters, and cloth coverings, is accomplished using high-volume, high-efficiency particulate air (HEPA) vacuum cleaners or by low-volume air sampling cassettes. The high-volume HEPA collection method utilizes a Dust Collection Filter Sock (Midwest Filtration Company, Fairfield, Ohio) to collect the sample for analysis. The low-volume air sampling cassette is a micro-vacuuming technique that collects the sample on a suitable filter substrate by pulling air through the filter contained in a closed-face, conductive sampling cassette by personal sampling or carbon vane pump.

\section{C.1.4.1 High-Volume HEPA Vacuum Sample Collection}

HEPA vacuum samples are collected as follows:

1. Insert a pre-weighed Dust Collection Filter Sock into a high-volume vacuum cleaner nozzle.

2. Vacuum the entire sample area with one pass by slowly moving the vacuum nozzle back and forth across the surface.

3. After sample collection, place the filter or sock in a pre-labeled, sterile, 50-ml Blue Falcon screw-top tube (Becton Dickinson Labware, Franklin Lakes, NJ), and sealed with a cap. 


\section{APPENDIXC}

4. After sealing, place in a sterile bag, seal it, and clean the outside of the bag with a 0.5- to 0.6-percent sodium hypochlorite solution.

5. Place the sealed sample inside an unused, self-sealing bag and ship according to applicable CDC guidelines and regulations. The following information, as well as an appropriate chain-of-custody is necessary for the acceptance of samples by the laboratory: discrete sampler identifier, sample location, size of the sampled area, sample type, time and date that the sample was taken, and name and initials of the person collecting the sample (CDC 2002).

\section{C.1.4.2 Low-Volume Vacuum Sample Collection}

Micro-vacuum sample are collected using an air sampling cassette with calibrated air flow at the maximum rate and using a short section of plastic tubing cut at a 45-degree angle attached to the inlet. A pre-weighed filter with a 0.5 - to 0.8 -micrometer $(\mu \mathrm{m})$ pore size is inserted into the cassette, and the air-flow rate is measured. Samples are collected as follows:

1. Make one pass of the entire sample collection area by slowly moving the vacuum nozzle back and forth across the surface.

2. After sample collection, place the filter in a pre-labeled, sterile, 50-ml Blue Falcon screw-top tube (Becton Dickinson Labware, Franklin Lakes, NJ), and sealed with a cap.

3. After sealing, place in a sterile bag, seal it, and clean the outside of the bag with a 0.5- to 0.6-percent sodium hypochlorite solution.

4. Place the sealed sample inside an unused, self-sealing bag, and ship according to applicable CDC guidelines and regulations. The following information, as well as an appropriate chain-of-custody is necessary for the acceptance of samples by the laboratory: discrete sampler identifier, sample location, size of the sampled area, sample type, time and date that the sample was taken, and name and initials of the person collecting the sample (CDC 2002).

\section{C.1.5 Air Sample Collection}

Air samples are collected to characterize suspended biological agents soon after a release or in the event of suspected resuspension. The time-since-release criterion is imposed because the biological agent concentration in air will decrease significantly over time as the agents settle out or disperse, thus limiting the value of air sample collection in undisturbed areas or in functioning ventilation systems. However, air sample collection at any time in areas where activity may resuspend agents may provide valuable information. Personal lapel air samplers should be worn by personnel working in potentially contaminated areas.

Air sample collection methods involve pulling volumes of air through a biological collection medium. The total air volume passing through the collection medium in air sample systems must be measured for accurate analysis of agent air concentration. The total air volume is measured as a function of calibrated air-flow rate and time.

\section{C.1.5.1 Cassette Sample Collection}

The cassette air sample collection system consists of an air pump, Tygon ${ }^{\circledR}$ tubing, and a three-piece 37-mm filter cassette constructed of conductive plastic material. The filter medium may be mixed polycarbonate, 


\section{APPENDIXC}

cellulose ester membrane, polytetrafluoroethylene, or gelatin filter with a pore size of 0.5 to 0.8 micrometers $(\mu \mathrm{m})$. A sample is collected as follows:

1. Insert a filter into the filter cassette and pull air through the filter for a minimum of six to eight hours at 2 to 4 liters per minute. Air flow rate is measured during the process. If gelatin filters are used, then an alternate sampling time and flow rate may be needed as specified by the manufacturer.

2. After the sample is collected, remove the filter, and place it in a labeled, clean, self-sealing bag.

3. After sealing, double-bag, seal it, and clean the outside of the bag with a 0.5- to 0.6-percent sodium hypochlorite solution.

4. Place the sealed sample bag inside another unused, self-sealing bag and ship according to applicable CDC guidelines and regulations. The following information, as well as an appropriate chain-ofcustody is necessary for the acceptance of samples by the laboratory: discrete sampler identifier, sample location, size of the sampled area, sample type, time and date that the sample was taken, and name and initials of the person collecting the sample (CDC 2002).

\section{C.1.5.2 Impactor Sample Collection}

Several commercial impactors are available with unique operating characteristics, such as particle-size cut points, operating flow rate, and collection media. Sample collection by impactor is accomplished by direct impingement of sample on nutrient agar for culture analysis or impingement of sample on filters for spore analysis or subsequent extraction and culture analysis.

For sample collection using filter media:

1. Insert filters into the impactor and measure air flow rate.

2. Collect the sample by pulling air through the filter media for a minimum of six to eight hours at two to four liters per minute.

3. After the sample is collected, remove the filter and place it in a labeled, clean, self-sealing bag.

4. After sealing, double-bag, seal it, and clean the outside of the bag and the impactor with a 0.5- to 0.6-percent sodium hypochlorite solution. The impactors should be autoclaved before use in an alternate site.

5. Place the sealed sample bag inside another unused, self-sealing bag and ship according to applicable CDC guidelines and regulations. The following information, as well as an appropriate chain-ofcustody is necessary for the acceptance of samples by the laboratory: discrete sampler identifier, sample location, size of the sampled area, sample type, time and date that the sample was taken, and name and initials of the person collecting the sample (CDC 2002).

For direct nutrient agar impingement:

1. Program the sampler for the volume of air to be sampled and aseptically place an open plate with appropriate agar growth medium in the sampler.

2. Collect the sample by pulling air through the impactor for a specified period of time.

3. Collect the plates and cover.

4. Seal plates with gas-permeable tape and transport to lab for incubation and plate count.

5. Ship according to applicable CDC guidelines and regulations. The following information, as well as an appropriate chain-of-custody is necessary for the acceptance of samples by the laboratory: 


\section{APPENDIXC}

discrete sampler identifier, sample location, size of the sampled area, sample type, time and date that the sample was taken, and name and initials of the person collecting the sample (CDC 2002).

\section{C.2 Extraction Methods}

Sample extraction is required to remove the biological agents from the collection medium in preparation for identification and enumeration. The extraction method must be vigorous enough to maximize agent removal but mild enough to limit impact on agent viability.

Wipe, swab, and filter samples are extracted by placing the wipe, swab, or filter into a fifty milliliter Blue Falcon screw-top tube (Becton Dickinson Labware, Franklin Lakes, NJ) containing twenty milliliters of 0.3 percent Tween 20 in phosphate-buffered saline solution (PBS) and vortexing for three minutes. The contents of the tube are allowed to settle for five minutes, and swabs and wipes are removed. The tube is then centrifuged at 3,000 to 4,500 revolutions per minute for fifteen to thirty minutes at $10^{\circ} \mathrm{C}$, the supernatant removed by decanting, and the pellet resuspended in two milliliters of PBS solution with 0.3 percent Tween 20 .

Vacuum sock samples are extracted by placing the sock into a fifty-milliliter flask containing twenty to thirty milliliters PBS with 0.3 percent Tween 20 and placed on a shaker for thirty minutes. After shaking, the contents of the flask are allowed to settle for five minutes, and the supernatant then poured into a fifty-milliliter Blue Falcon screw-top tube (Becton Dickinson Labware) and centrifuged at 3,000 to 4,500 revolutions per minute for fifteen to thirty minutes at $10^{\circ} \mathrm{C}$. Following centrifugation, the supernatant is removed by decanting, and the pellet is resuspended in two milliliters PBS with 0.3 percent Tween 20.

\section{C.3 Surfaces and Structures Encountered}

Indoor surfaces found in a typical transportation facility include both porous and nonporous surfaces, such as, metal, tile, wallboard, paint, furniture, carpet, and ventilation system filters, which necessitate the use of both bulk and surface collection methods. The Table C-1 presents the best available collection methods for each identified surface type. 


\section{APPENDIXC}

Table C-1. Available collection methods for surfaces.

\begin{tabular}{|l|l|}
\hline \multicolumn{1}{|c|}{ Surface Type } & \multicolumn{1}{c|}{ Collection Method } \\
\hline Duct work & Bulk \\
\hline HVAC filter & Bulk, vacuum \\
\hline Electronic equipment & Swab \\
\hline Paper & Swab \\
\hline Glass & Wipe \\
\hline Stainless steel & Wipe \\
\hline Glazed ceramic tile & Wipe \\
\hline Vinyl & Wipe \\
\hline Plastic laminate & Wipe \\
\hline Painted wallboard & Wipe, vacuum \\
\hline Painted concrete & Wipe, vacuum \\
\hline Marble & Wipe, vacuum \\
\hline Terrazzo & Vacuum \\
\hline Cloth & Vacuum \\
\hline Carpet & Vacuum \\
\hline Nonglazed ceramic tile & Vacuum \\
\hline Nonpainted wallboard & Vacuum \\
\hline Nonpainted concrete & Vacuum \\
\hline Acoustic tile & Vacuum \\
\hline
\end{tabular}

\section{C.4 Analytical Methods}

Two screening assays are useful to determine initially and rapidly the presence of $B$. anthracis during the early phases of a biological incident. These methods are an immunoassay device [e.g., BioThreat Alert Test Strips, Alexeter LLM, Chicago IL; RAMP Anthrax Test Cartridge (see http://www.aoac.org/DHS_release. pdf)] for visible powders only and a nucleic acid analysis technique (TaqMan PCR). The immunoassay can be used for presumptive identification, followed by the more specific nucleic acid test. However, to confirm the presence of $B$. anthracis, and during characterization and clearance sampling, culture identification is the gold standard and can yield important strain information. A key reason for the use of culturing is that the limit of detection of culture (namely, one recovered viable spore resulting in one visible colony) is lower than for either immunoassays or PCR-based methods. In addition, although PCR is quite sensitive and specific, it is susceptible to "poisoning" by background environmental dust and contamination that can result in false negatives, whereas culture analysis appears to be unaffected by such background material (Buttner et al. 2004b).

A sample contained in two milliliters of PBS solution with 0.3 percent Tween 20 can be split three ways for each analysis method. The culture and TaqMan PCR analytical methods are performed by experienced technicians at a public health laboratory as part of the Laboratory Response Network. The closest Laboratory Response Network lab to San Francisco International Airport is the California Department of Health Services at 850 Marina Bay Parkway in Richmond, California (phone 510-307-8575). The protocols that follow should be performed by trained personnel. 


\section{APPENDIXC}

\section{C.4.1 Immunoassays}

Immunoassays are used in the field for only visible powders to quickly determine if a biological warfare organism is present. These tests, commonly referred to as immunoassays, rely on an antibody (biological recognition molecule) that only reacts to a unique antigen when present. Antibodies can detect antigens found on the surfaces of bacterial cells and spores with about a three percent false positive rate. Emergency personnel can perform these tests onsite; however, a trained technician should be available to interpret the results of the test. Whole cells are used for this procedure, so after a sample is retrieved from the sample collection medium (wipe, swipe, or filter), it is ready to use.

\section{C.4.2 DNA-Based Methods}

This section is included to give emergency personnel an idea of the advanced DNA techniques used to verify a biological warfare event. DNA-based methods are performed by experienced technicians at public health laboratories that are part of the Laboratory Response Network. The closest Laboratory Response Network lab to San Francisco International Airport is the California Department of Health Services at 850 Marina Bay Parkway in Richmond, California (phone 510-307-8575).

\section{C.4.2.1 DNA Extraction Protocol for High-Throughput Processing (Laboratory Response Network, BioWatch)}

After samples have been extracted from their sampling medium (filter, wipe, swipe) into 2-milliliter, 0.3 percent Tween 20 in PBS solution, 600 micro liters of sample is removed using an autoclaveable 1000 micro liter pipettor and placed into a gasketed, capped tube containing 50 milligrams of 500 micron and 50 milligrams of 106 micron, acid-washed glass beads. The tube is inserted in a "Mini Bead Beater" instrument (or bead-beating instrument that accommodates a robot tube rack) and the sample(s) is bead beaten for 3 minutes at 5000 revolutions per minute. This disrupts cells and releases DNA.

The tube is then placed on ice to cool for 1 minute, transferred to a robot tube holder and then centrifuged for approximately 30 seconds at 2000 revolutions per minute. This separates beads and vegetative material from the DNA. The tube holder containing the samples is loaded on a robot, which transfers 400 microliters of the supernatant from the tubes to a 96-well multi-screen filter plate for high throughput separations (0.22 micron MAGVN, Millipore). Using the robot programming, the tubes are tracked to their respective wells in the 96-well plate.

Upon completion of the transfers, the robot puts the filtration plate on a suction manifold and the samples are filtered through to a second 96-well filtration plate (100 kd MANU polymerase chain reaction cleanup plate, Millipore). The samples are taken through three $1 \mathrm{X}$ TE washes and a final polymerase chain reaction water wash on the same manifold. The final extract samples are transferred by robot to a 96-well, thin-wall, polymerase chain reaction, thermal cycler plate. Sample extracts then go through real polymerase chain reaction analysis using 96-well format polymerase chain reaction instrumentation.

\section{C.4.2.2 TaqMan Polymerase Chain Reaction}

Real-time polymerase chain reaction assays are performed for each sample on an ABI Prism 7000 Sequence Detection System (Applied Biosystems, Foster City, CA). An inhibition control consisting of DNA from the 


\section{APPENDIXC}

processed filter section and positive control DNA, a positive control DNA, and a no-template control are performed for each test assay.

Prior to each day's testing, positive control DNA is run in all instrument wells to verify functionality. Results are reported as cycle threshold values, which correspond to the number of polymerase chain reaction cycles performed when DNA is detected above a specific threshold. Laboratory Response Network standard protocol cycle conditions are used, and reaction components consist of (final concentrations): $6 \mathrm{mM} \mathrm{MgCl}_{2}$, $0.2 \mathrm{mM}$ each of ATP, GTP, CTP, and TTP, $20 \mu \mathrm{g}$ bovine serum albumin, 20 pmole $/ \mu \mathrm{L}$ of each Yersinia pestis or B. anthracis primers and their respective Yersinia pestis or B. anthracis TaqMan ${ }^{\circledR}$ probe, and 1.25U Platinum Taq DNA Polymerase (Gibco-BRL, Gaithersburg, MD) in 1X Platinum Taq PCR Buffer without $\mathrm{MgCl}_{2}$ and polymerase chain reaction water (Teknova, Half Moon Bay, CA) in $25 \mu \mathrm{L}$ final volume. Polymerase chain reactions are one cycle of one minute at $95^{\circ} \mathrm{C}$, followed by forty-five cycles of fifteen seconds at $95^{\circ} \mathrm{C}$ and fifteen seconds at $60^{\circ} \mathrm{C}$.

\section{C.4.3 Culture-Based Methods}

The culture-based assay is essential for confirming the presence and viability of $B$. anthracis spores used in a bioterrorist attack. All culture studies must be performed at a BSL-2 facility by trained personnel only. The closest Laboratory Response Network lab to San Francisco International Airport is the California Department of Health Services at 850 Marina Bay Parkway in Richmond, California (phone 510-307-8575).

At a BSL-2 Laboratory, samples are streaked onto Brain Heart Infusion Agar, blood agar, and a selective medium if the isolation process is overrun by contaminating background microorganisms. The streaked plates are incubated $35^{\circ} \mathrm{C}$ to $37^{\circ} \mathrm{C}$ in ambient air and examined after forty-eight hours. Results are reported as number of colony forming units per plate. Standard bacterial identification tests are performed to verify that growth colonies are B. anthracis. 


\section{APPENDIX C}

\section{C.5 Materials Vendors}

\begin{tabular}{|l|l|}
\hline \multicolumn{1}{|c|}{ Type of Material } & \multicolumn{1}{c|}{ Vendors } \\
\hline Wipes and swabs & $\begin{array}{l}\text { Wipe pads, } 5.0 \times 5.0 \text { cm sterile rayon, Dukal Corporation, Syosset, NY } \\
\text { Swabs, sterile rayon, Environmental Swab Kits from CDC, Atlanta, GA }\end{array}$ \\
\hline Vacuums and filters & $\begin{array}{l}\text { Light-weight HEPA vacuum cleaner, Atrix International Inc, Burnsville, MN } \\
\text { HEPA vacuum sock, Midwest Filtration Co, Fairfield, OH } \\
\text { Aerosol cassette, SKC, Houston, TX } \\
\text { Aerosol cassette pump, SKC, Houston, TX } \\
\text { Polytetrafluoroethylene (PTFE) 0.5 } \mu \text { m, Millipore, Billerica, MA }\end{array}$ \\
\hline Impactor and Aerosol & $\begin{array}{l}\text { BioCassettes with BHI agar, SKC, Houston, TX } \\
\text { Viable Bacteria Impactor with BHI agar, N6, Andersen Samplers Inc., Atlanta, GA } \\
\text { Cassette Air Sampler, 6-stage, Andersen Samplers Inc., Atlanta, GA } \\
\text { Aerosol cassette, SKC, Houston, TX } \\
\text { Aerosol cassette pump, SKC, Houston, TX }\end{array}$ \\
\hline Wetting Agents & $\begin{array}{l}\text { Deionized water, Sigma Chemical Co., St. Louis, MO } \\
\text { Phosphate-buffered solution (PBS), Sigma Chemical Co., St. Louis, MO } \\
\text { Tween 80 (polyoxyethylene sorbitan monooleate), Sigma Chemical Co., St. Louis, MO }\end{array}$ \\
\hline Tubes & Blue Falcon screw-top tube, 50 ml, Becton Dickinson Labware, Franklin Lakes, NJ \\
\hline Immunoassay & Biothreat Alert Test Strips, Alexeter Technologies LLC, Chicago, IL \\
\hline BHI agar plates (catalog \# B1010), Teknova, Inc., Half Moon Bay, CA \\
Blood agar plates (catalog \# B0142), Teknova, Inc., Half Moon Bay, CA \\
Pipettor, autoclaveable, Fisher Scientific, Atlanta, GA \\
Pipette tips, sterile disposable, Fisher Scientific, Atlanta, GA
\end{tabular}




\section{APPENDIX C}

\section{C.6 References}

ASTM (2006), Standard E 2458-06, "Standard Practices for Bulk Sample Collection and Swab Sample Collection of Visible Powders Suspected of Being Biological Agents from Nonporous Surfaces."

Buttner, M. P., P. Cruz, L. D. Stetzenbach, A. K. Klima-Comba, V. L. Stevens, and P. A. Emanuel (2004a), "Evaluation of the Biological Sampling Kit (BiSKit) for Large-Area Surface Sampling," Appl. Environ. Microbiol. 70(12), 7040.

Buttner, M. P., P. Cruz, L. D. Stetzenbach, A. K. Klima-Comba, V. L. Stevens, and, T. D. Cronin (2004b), "Determination of the Efficacy of Two Building Decontamination Strategies by Surface Sampling with Culture and Quantitative PCR Analysis," Appl. Environ. Microbiol. 70(8), 4740.

CDC (2002), Centers for Disease Control and Prevention, Comprehensive Procedures for Collecting Environmental Samples for Culturing Bacillus anthracis; available at http://www.bt.cdc.gov/Agent/ environmental-sampling-apr2002.asp.

Hung, L.-L., J. D. Miller, and H. K. Dillon (2005), Field Guide for the Determination of Biological Contaminants in Environmental Samples, $2^{\text {nd }}$ Edition, American Industrial Hygiene Association, Fairfax, VA. Rose, L., B. Jensen, A. Peterson, S. N. Banerjee, and M. J. Arduino (2004), "Swab Materials and Bacillus anthracis Spore Recovery from Nonporous Surfaces," Emerg. Infect. Dis. (serial on the Internet); available at http://www.cdc.gov/ncidod/eid/vol10no6/03-0716.htm.

Sanderson, W. T., M. J. Hein, L. Taylor, B. D. Curwin, G. M. Kinnes, and T. A. Seitz (2002), "Surface Sampling Methods for Bacillus anthracis Spore Contamination, Emerging Infectious Diseases Journal 8(10). 



\section{Considerations for Sampling Design}

This appendix describes general considerations for sampling design applicable to airports and to both characterization environmental sampling and clearance environmental sampling. Specific details of any characterization or clearance sampling design, including how many samples to take and where to take them, are developed in the context of issues discussed in this appendix.

\section{D.1 Purposes of Sampling}

Two major types of environmental sampling are conducted during airport cleanup activities. Characterization environmental sampling gathers information about the contamination. Clearance environmental sampling assesses the success of decontamination. The templates in Appendixes $\mathrm{H}$, I, and $\mathrm{K}$ are designed to help develop sampling plans and document work as it is done.

\section{D.2 Pre-Planning}

Because an airport is a complex physical environment, it is advantageous to plan ahead for the kinds of sampling that would be done in the event of a biological warfare agent (BWA) attack. Much of the planning is based on the physical structure of an airport. Providing the cleanup team with a list of potential sampling zones and sampling units will save time. Providing a complete list will help ensure that every area, and everything in every area, is at least considered for sampling even if not actually sampled. Thoroughness will increase confidence in the decisions that must be made. It is imperative that the person responsible for the sampling design visit the sample locale and fully evaluate the physical nature of areas to be sampled before designing the sampling plan. Such a visit will reduce the possibility of producing a sampling plan that may have potentially dangerous consequences or be impractical to implement.

\section{D.2.1 Physical Structure}

Beyond basic structures such as floors, walls, and ceilings, airports contain many objects of widely varying size, shape, and construction. Examples include ticket counters, flight arrival and departure monitors, information signs, escalators, seats, conveyor belts, metal detectors, scales, artwork, and kiosks. Cooling fans that pull air through objects can cause the deposition of contaminant inside the objects (examples include monitor banks, refrigerators, and museum display cases). Ticket counters with drawers and cupboards can potentially be contaminated on both outside and interior surfaces. Semi-industrial portions of an airport have machine rooms with various kinds of mechanical and electrical equipment, baggage handling equipment, and heating and cooling systems. Standard office spaces have typical office equipment, such as desks, computers, and copiers.

San Francisco International Airport (SFO) is similar to most airports in that most walls are not large, flat areas but are sectional, with joints between sections. Various types of seams, joints, beams, and girders interrupt the continuity of walls, windows, and ceilings. Small cavities within seams and joints may serve as reservoirs for BWAs. Data Supplement A (available by request to SFO) includes detailed information for SFO.

Some airport surfaces are smooth, and many different types of materials are used, including metal, plastic, tile, and painted or unpainted gypsum wallboard. Textured surfaces include carpeting on floors and some walls. Structures that do not have large, flat surfaces include baggage transport systems, escalators, and 


\section{APPENDIX D}

baggage screening units. To manage the sampling of such a complex environment, an airport can be hierarchically partitioned into sampling zones and sampling units.

\section{D.2.2 Sampling Zones}

A sampling zone is a discrete portion of an airport within which sampling is conducted. Zones should be chosen in a way that helps remediation planners and workers keep track of what sampling has been done, where it has been done, and what information has been acquired from sampling.

Potential sampling zones should be chosen before an incident on the basis of an airport's physical structure. Separate sampling zones are likely to be those sections of an airport that are physically separate in some manner. Boundaries between zones should be accurately described and easy to find.

A useful way to identify potential sampling zones is to begin with the entire airport and then break it down into successively smaller areas until manageable and sensible sampling zones are defined.

Each airport can initialy be partitioned into terminal buildings and one or more boarding areas. Each floor can be considered separately. For each floor, air supply or life safety zones - defined by the ventilation and fire safety design of the facility - can provide the next level of partitioning.

For example, the small corridors that may separate main terminal buildings make sensible sampling zones. These corridors are also a good location for constructing barriers that would allow opposite sides to be decontaminated separately, or allow only one side to be decontaminated if such a decision were made. In other words, their utility with respect to decontamination decisions makes the corridors both logical and practical sampling zones.

Following a BWA event, pre-identified sampling zones should be reviewed and possibly modified in light of any details about the event. Information specific to the incident may be known (e.g., from witnesses or monitors) or inferred (e.g., from epidemiological results implicating a certain boarding area, or models of agent spread). Given such information, changes to sampling zones might take into account areas with the greatest potential exposure (even if expected concentrations are low) and areas of greatest expected concentration (even if exposure potential is low).

One of the main purposes of sampling is to provide data to support decisions. Therefore, potential sampling zones should also take into account how decision-making might be organized. At SFO for example, is likely that decisions about whether and how to decontaminate various boarding areas would be made separately. Thus, it would not make sense to consider them as the same sampling zone. Data Supplement E identifies potential sampling zones for selected portions of SFO.

\section{D.2.3 Sampling Units}

Sampling units are surfaces, objects, or sets of objects within a sampling zone that can be sampled. As a general rule, each sampling unit should be a physically homogenous area, object, or set of objects about which it is reasonable to make a single, collective assessment or decision. For example, a set of ticket counters is homogenous in the sense that they are all ticket counters, and all are built the same way. As a set, they can be a single sampling unit if it is reasonable to make a single assessment or decision about all of them. It would not make sense to make a single, collective decision about the ticket counters together with nearby CTX machines whose purpose is to screen oversized baggage. Thus, ticket counters and baggage screening units should be separate from the point of view of sampling design. Within a sampling zone, 


\section{APPENDIX D}

floors and walls should be considered separately because they contribute differently to potential exposure. Thus floors, walls, ticket counters, and CTX machines are four different sampling units.

Pre-identified sampling units should be reviewed and adjusted on the basis of event-specific information. For example, if the location of a release is known, that information combined with known airflow patterns might indicate that some regions of the floor are more likely to have contamination than others. The floor could be partitioned into sub-units on that basis. If dispersion modeling identifies a region of highest expected concentration, that region would likely be a sampling unit. Moreover, any information about ways in which decontamination tends to fail should be incorporated in the clearance sampling design. For example, if fumigation tends to be poor in corners where floors meet walls, the corners could be defined as a separate sampling unit. This kind of partitioning should continue until the sampling design team decides that reasonable and manageable sampling units are defined.

Sampling units should be contained entirely within sampling zones. Sampling zones, in turn, are located within characterization zones or clearance zones, which are defined in Section D.2.5.

Identifying potential sampling units ahead of time yields two main benefits. First, it allows personnel to plan ahead for the variety of sampling methods that might be used. For example, carpets cannot be effectively sampled with wipes, so knowing that carpets may need sampling informs planners that vacuum sampling will probably be needed in addition to wipe sampling. Second, it helps ensure that everything that might be sampled is at least considered for sampling, even if not actually sampled.

\section{D.2.4 Selecting Sampling Locations}

Given a complete list of sampling units, the choice of which ones to sample should be based on the questions that sampling is expected to answer, the hypotheses that sampling information will be used to test, or the decisions that sampling is expected to support.

Following are some of the more important potential questions that characterization sampling is expected to answer:

- Is the agent present in places likely to involve human exposure?

- What are the highest surface concentrations of contamination?

- How did contamination reach an area?

- Is the agent re-aerosolizable (if not determined during First Response)?

In broad terms, the following major decisions require support from environmental sampling data:

- Whether or not to decontaminate.

- If decontamination is necessary, where to decontaminate.

- If decontamination is necessary, how to decontaminate.

- After decontamination, whether decontamination was successful.

Potential sampling locations should be assessed in terms of their likelihood to contribute answers to these kinds of questions and decisions. (Note that many other questions may arise during a real event. The list of questions, above, is not intended to limit remediation planners from raising additional questions.) In addition, it may be advantageous during characterization to find contaminated locations so that comparisons of sampling results can be made for such locations before and after decontamination. 


\section{APPENDIX D}

Following are some suggested categories of items and related issues that can help guide the selection of sampling locations:

- Locations likely to be touched by humans, including handrails, chairs, benches, waste containers, counters, drinking fountains, telephones, vending machines, desks, cash registers, computers, and ticket counters. Walls, windows, and other accessible vertical surfaces up to about 8 feet above the floor.

- Floors, taking into account the different types of floor surfaces.

- Upward-facing surfaces other than floors, including shelves, ledges, windowsills, internal low walls, tops of ticket counters, tops of tables, and tops of desks.

- Vertical surfaces out of reach, including walls and windows above 8 feet from the floor.

- Locations where a BWA might be likely to remain after deposition (repositories). This is especially important after a covert release because of the delay between release and sampling. Examples include surfaces with electrostatic charge (e.g., CRT screens not protected from contact by transparent plastic), tops of light fixtures, tops of signs; air ducts and surfaces near air-supply registers; air return registers, plenums, and air-intake grills that are part of equipment cooling systems (e.g., on CRT cabinets); miscellaneous nooks and crannies.

- Locations with a known relation to a means by which the agent spreads. For example, floors near air supply registers if it is believed that the agent entered the heating, ventilation, and air conditioning (HVAC) system while it was operating; areas where people went after passing through a source area.

- Air sampling, which can reveal whether or not reaerosolization is occurring. For B. anthracis it is important to find out whether aerosolization of spores occurred or could occur (Weis et al. 2002). If disease has not been observed, it is important to sample return air grills, tops of ceiling fans and light fixtures, high window sills, and the like. Absent positive samples from such locations, the presumption is that aerosolization of spores did not occur. Additional data on the physical and chemical properties of spores also plays a role in decisions about aerosolization.

- Surface samples from areas of expected deposition or greatest predicted concentration, and in areas of greatest exposure potential. Such areas can be identified using either a conceptual or mathematical airflow model. Locations with exposure potential include objects likely to be touched by humans (see the first item, above, in this list).

- High-traffic areas to identify potential locations of cross-contamination. Specific information about where people may have tracked the agent after its release might be available from security cameras or post-event interviews.

- Surface samples of the HVAC system, including air filters, baffles, and other surfaces within the system.

A grid-based or geostatistical sampling scheme can be used to develop contaminant maps. Such maps can help highlight patterns of contamination, can validate a single dispersion model or select among alternative dispersion models, and can identify approximate boundaries of contaminated areas. An electronic datacollection and data-management tool featuring electronic facility drawings, bar code tracking, and data visualization [called the Building Restoration Operations Optimization Model (BROOM)] is under development and expected to be available soon to help manage future characterization data. 


\section{APPENDIX D}

\section{D.2.5 Other Types of Zones}

Several other kinds of zones are useful for organizing airport cleanup. A characterization zone is a discrete section of an airport that is viewed as a whole from the point of view of characterization. Decontamination zones are areas in which decontamination will take place. If the decontamination method were fumigation, then the term fumigation zone would be used instead. A clearance zone is a portion of the facility in which a clearance decision is made, and made separately from other portions of the facility.

\section{D.3 Characterization Sampling}

Characterization sampling takes place after a BWA event has been confirmed and after first-response actions are complete. The purpose of characterization is to gather information necessary for the subsequent phases of cleanup.

\section{D.3.1 Characterization Sampling for Decontamination Design}

With respect to decontamination, characterization sampling helps answer the question: Does each sampling zone need to be decontaminated (yes or no)?

- If yes, how?

If fumigation is to be used, are there any areas within the zone that should receive surface decontamination prior to fumigation?

If fumigation is to be used, what fumigant level (concentration $\times$ time) needs to be maintained?

If fumigation is not to be used, what areas need decontamination (presumably by surface decontamination)?

- If no, is there sufficient confidence in that decision?

The Technical Working Group (TWG) must choose how extensively to sample. The decision may depend on the nature of the contaminant and anticipated method of decontamination. For example, the TWG may decide that every sampling unit that might have been contaminated — and if contaminated, presents an exposure hazard - must be sampled. In contrast, if fumigation is the method of choice for the agent, the TWG may choose a less-conservative approach. For example, it may decide that as soon as contamination within a sampling zone is confirmed by any means, no further sampling in that zone is needed to decide whether or not to fumigate the zone. On the other hand, if fumigation is not anticipated, and all decontamination will be done by surface decontamination, then characterization sampling must be designed to find all areas that need washing. 


\section{APPENDIX D}

If the BWA is easily dispersed and if it can easily be resuspended which allows it to travel to new locations, then decisions about the extent of sampling must take into account ways that resuspension and redistribution could take place. A BWA that requires special environmental conditions for survival (such as warm, moist conditions) might be presumed dead after some time in locations without those conditions. Sampling may not be required in such locations.

It is less expensive to replace certain objects than to characterize, decontaminate, and clear them. The amount of sampling will be reduced if such objects are removed and disposed regardless of whether they are contaminated. Lists of such objects should be prepared before an event.

Characterization sampling can be iterative. For example, areas initially found to be highly contaminated might be more extensively sampled. Additional sampling could be conducted to better define the boundaries of contaminated areas. Then areas that were omitted during the first characterization sampling would be sampled.

The decision regarding which sampling strategy (judgmental or statistical, see Section D.6) to use may be different for different sampling units or for classes of sampling units, and such decisions may change. For example, if initial judgmental characterization sampling indicates the need for more precise, thorough, or statistically representative information, then statistically designed characterization sampling may be required.

\section{D.3.2 Characterization Sampling To Prepare for Clearance}

A second use of characterization sampling is to provide initial data that can be compared with subsequent data obtained during the clearance process. Locations identified as contaminated during characterization are sampled again during clearance to find out if decontamination at the locations is successful. In the context of cleanup after a BWA event, this sampling approach has become known as "targeted" sampling.

For agents that aerosolize, a clearance decision will be based on surface sampling results followed by aggressive air sampling (see Section 4.2.1 in the main text of this Remediation Guidance document). If the portion of a clearance decision that is based on surface samples uses only resampling of locations found to be contaminated during characterization, then it is especially important that characterization be thorough with regard to spatial extent. If characterization sampling is not extensive, there will probably be contaminated areas that were not sampled, and thus not re-sampled. Insufficient characterization sampling increases the chance that clearance sampling will fail to sample an area where decontamination failed.

\section{D.4 Clearance Sampling}

The purpose of post-decontamination clearance sampling is to decide whether or not decontamination was successful. The definition of "success" requires some care. The most general definition is that decontamination reduced contamination to acceptable levels. In the remediation of facilities contaminated with $B$. anthracis in 2001 , the acceptable level was no growth on any clearance environmental sample. That is, any level that is not detectable is acceptable. The acceptable level must be defined before any clearance sampling takes place. When statistically designed sampling is used, the definition of acceptable level is an essential input to the statistical design. A sampling strategy depends on both the definition of success and on the way results from the sample locations are used to infer levels in nonsampled locations (See Sections D.5 and D.6.). 
If fumigation is used, then every fumigation zone requires clearance sampling. Therefore, every sampling zone within the fumigation zone must be sampled. If fumigation is not used, then every area that was decontaminated (e.g., every area subjected to surface decontamination) requires clearance sampling. This statement presumes that areas not subjected to surface decontamination are within acceptable levels, as determined during characterization.

To the extent possible, the same sampling units should be used during clearance sampling as during characterization sampling. It is possible that information gathered during characterization may cause the definitions of sampling units to be refined for clearance sampling. It is undesirable to completely redefine sampling units because a redefinition would make before-and-after comparisons difficult. Reasonable changes to sampling unit designations might consist of either subdividing sampling units (e.g., when contamination was found, and a more detailed assessment is anticipated during clearance) or combining two or more sampling units into a single, larger sampling unit (e.g., when no contamination was found in any units during characterization).

The number of samples and their locations may change from characterization to clearance sampling, especially if statistical sampling is used. However, judgmental sampling performed during characterization should be repeated during clearance, especially sampling in the areas of greatest exposure potential and greatest expected concentration (see Section D.6.3).

Any clearance sampling used for before-and-after comparison of specific locations must take place before aggressive air sampling begins. Aggressive air sampling (see Section 4.2.1 of the main text) is done to resuspend particles, such as B. anthracis spores, so that they can be collected in air samples. After resuspension, particles will settle in new locations, and any before-and-after comparison with characterization results is invalid.

\section{D.5 Decision Uncertainty}

The primary purpose of clearance sampling is to support a decision: Should or should not the facility be cleared for reuse? Several factors contribute to uncertainty in making this decision.

Suppose it is agreed that a facility may be reopened when "no growth of viable spores is found in any clearance sample." This is a surrogate for a more important goal: that the facility is safe to use. To take an extreme and unrealistic example, a clearance decision based on a single sample would have no credibility whatsoever because it would be far too easy for a single sample to miss residual contamination (i.e., contamination still present after decontamination) in locations that would make the facility unsafe to use. As the number of samples increases, it becomes more likely that residual contamination, if any, will be found. However, unless every sample that possibly could be collected has been collected, there is always a possibility that some residual contamination is present at a nonsampled location. To make matters worse, the smaller the area(s) of residual contamination, the less likely it will be found during clearance sampling.

Another factor that contributes to decision uncertainty is the fact that currently available sampling methods do not have $100 \%$ efficiency, that is, they do not necessarily pick up all of the spores that might be present. Thus, viable spores could be present in the facility, but not found in the sample even when the sample was collected in a location where spores are present. Yet another factor is the limit of detection of the analytical method. Even when viable spores are collected by the sampling material, the analytical method may not reveal their presence if there are too few of them. For all of these reasons, some uncertainty is always associated with a decision to reopen a facility. 


\section{APPENDIX D}

\section{D.6 Choosing Sample Locations}

There are several possible methods for choosing sample locations and a variety of names for the methods. They include:

- Exhaustive sampling.

- Judgmental sampling.

- Targeted sampling.

- Biased sampling.

- Random sampling.

- Random (only) sampling.

- Statistical sampling.

The distinction between biased and targeted, as types of judgmental sampling, has been used in recent anthrax cleanups, including the Senate Hart building and State Department mail annex.

\section{D.6.1 Exhaustive Sampling}

Exhaustive sampling occurs when every sample that could possibly be collected is actually collected. Exhaustive sampling requires sampling every part of a sampling unit and then analyzing every sample with no errors by the analytical laboratory, with no lost samples, with no data transcription errors, and with no other errors. Such sampling has the benefit of reducing decision uncertainty arising from the selection of sampling locations.

Sampling an entire 30,000- $\mathrm{ft}^{2}$ surface with 4-in ${ }^{2}$ wipe samples, for example, would require more than 1 million wipes on the floors alone. Even if resources were available to collect and analyze so many samples, doing so without error is extremely unlikely. For all practical purposes, it is not possible to perform exhaustive sampling of large surfaces with wipe samples. Swab samples have the same limitation. Exhaustive sampling of a small number of small surfaces, such as drinking fountains, might be practical, although the potential for errors still exists.

In contrast, it is possible for a single vacuum sample to generate a result that is representative of a large surface area. It may be possible, for surfaces at least, to use vacuum methods to come close to exhaustive sampling; however, such sampling would not be truly exhaustive unless it was also completely error-free. Because exhaustive sampling is difficult to fully achieve, prohibitively expensive, or both, it is not likely to take place during cleanup of an airport.

\section{D.6.2 Judgmental Sampling}

Judgmental sampling is a general term for the practice of choosing to sample specific locations for specific reasons. Crucial to the use of data resulting from judgmental sampling is the recognition that its value and success depend on the good judgment of individuals choosing the locations to be sampled, and on the quality of any information used as a basis for their choices. It is essential that those individuals have appropriate background, training, and experience. 


\section{D.6.3 Targeted and Biased Sampling}

The terms "targeted" and "biased" sampling are used during clearance sampling. Targeted sampling refers to re-sampling, during clearance, areas that were found during characterization to be definitely contaminated. It also refers to re-sampling, during clearance, not just the same area, but exactly the same contaminated locations that were sampled during characterization. Biased sampling refers to sampling, during clearance, areas that were found during characterization to have some contamination, or are near an area of definite contamination.

For example, in the event of a well-defined point release of BWA, a likely scenario would be heavy contamination close to the point of release and some contamination nearby. Presuming that the characterization process is precise enough to distinguish between areas of heavy contamination and surrounding areas with some contamination, the two would become the areas of targeted and biased sampling, respectively, during clearance.

\section{D.6.4 Random Sampling}

Random sampling refers to any method that includes randomizing the sample locations. The advantage of random sampling is that it is not subject to inadvertent or unconscious bias from the sampling design team. When the criteria for choosing the number of samples is based on a probability model of the outcome, the sampling design in addition to being random is also statistical (see Section D.6.4.2). Otherwise, the sampling is random but not statistical.

\section{D.6.4.1 Random (Only) Sampling}

The following is an example of sampling that is random but not statistical. Partition a large surface into $1000-\mathrm{ft}^{2}$ grid cells. Then within each cell collect a single $100-\mathrm{ft}^{2} \mathrm{HEPA}$ vacuum sample at a random location, so that $10 \%(100 / 1000)$ of the total surface area is sampled. If the coverage $(10 \%)$ is chosen arbitrarily and not based on any probability-of-detection calculation, then the sampling is random, but not statistical. Random (only) sampling is valuable and may have a very good chance of detecting a failed decontamination, depending on the way in which decontamination failed.

\section{D.6.4.2 Statistical Sampling}

Statistical sampling is used when a quantitative estimate of uncertainty is desired for the results from sampling. For example, decision-makers might want to be able to say, "We are $90 \%$ confident," rather than, "We are highly confident." Uses of statistical sampling include:

- Sampling to estimate a population parameter, such as the average surface concentration or the percentage of area above a risk-based concentration threshold.

- Sampling to yield a high probability of detecting residual contamination, if any.

- Sampling to confirm that little or no residual contamination is present.

For example, in the first approach, above, if the average concentration of agent (in appropriate measurement units) on the floor is part of the clearance criteria, statistical sampling methods can provide an estimate of the uncertainty of the measured average, and therefore a measure of confidence about whether or not the 


\section{APPENDIX D}

average level is acceptably low. At the current time, however, nonzero thresholds for acceptable residual B. anthracis contamination (i.e., contamination still present after decontamination) do not exist.

In the second approach, above, statistical methods can be used during clearance sampling to ensure a known (and presumably high) probability of detecting residual contamination, if any. Then, if residual contamination were not found, there would be some sense of confidence that residual contamination is not present at all (but the level of confidence would not be quantified). For example, the number of samples could be based on a requirement similar to the following: If in fact $3 \%$ or more of the surface area has viable B. anthracis present in concentrations that are detectable, then there must be a $95 \%$ probability that at least one clearance sample will be placed within that $3 \%$ of the area.

In the third approach, if no residual contamination is found, an upper confidence limit on the amount of residual contamination is calculated. In some cases, sampling can be designed so that the upper confidence limit is zero, thus providing a quantified confidence that there is no residual contamination present.

Statistical sampling is also appropriate when the source of contamination is unknown and there is no basis for using targeted sampling.

To use statistical sampling, some statistical design information is required. For clearance sampling, the information includes specifying the maximum acceptable level of residual contamination and the acceptable levels of decision uncertainty.

Statistical sampling always includes some degree of randomization of sampling locations, so statistical sampling is possible only in sampling units for which randomization is practical. Such units might include floors, walls, windows, or ceilings. Complex items, such as baggage screening machines, are not well suited to statistical sampling. For additional discussion of the statistical models used to design statistical sampling, see Appendix E.

\section{D.7 Representative and Reproducible Sampling}

The goal of both characterization and clearance environmental sampling is to produce results that are representative of the sampling unit from which they were collected. If samples are not representative, then decisions based on the results of those samples might be valid for specific locations actually sampled, but they would not be valid for the sampling unit as a whole. This situation could lead to a decision to reopen the airport when it is not, in fact, safe to do so.

For random and statistical sampling, representative means that the characteristics of the samples (such as average concentration, percentage of detections, and approximate range of concentrations) are unlikely to be much different from the characteristics of the sampling unit as a whole. Representative samples are obtained by randomizing sample locations. Randomization makes it unlikely that all of the samples will be collected from locations with extreme or unusual degrees of contamination.

For judgmental sampling, representative means that the samples were collected in a sufficient number of appropriate locations such that valid inferences can be made about the sampling unit as a whole. Representative samples can be obtained when there is sufficient knowledge of the likely locations of contamination and sufficient knowledge to be reasonably confident that there is either no contamination or negligible contamination at nonsampled locations. During clearance sampling, for example, if areas known to have originally had the worst contamination are found to be clean, then it might be reasonable to infer that other areas are also clean. 


\section{APPENDIX D}

Reproducible means that, if the entire sampling process were to be repeated, the results would not be much different. Repeating the entire sampling process includes, in the case of statistical sampling, randomly choosing sample locations, which would result in different locations being sampled.

\section{D.8 References}

Weis, C. P., A. J. Intrepido, A. K. Miller, P. G. Cowin, M. A. Durno, J. S. Gebhardt, and R. Bull (2002), "Secondary Aerosolization of Viable Bacillus anthracis Spores in a Contaminated U.S. Senate Office," JAMA 288 (22), 2853. 



\section{APPENDIXE}

\section{Probability-Based Sampling}

This appendix presumes that environmental sampling is organized by sampling zones, as described in Sections 2.2.6 and D.2. Within each sampling zone are one or more potential sampling units, such as the floor, walls, and other objects (see glossary at the end of Appendix E). Each sampling unit is considered for sampling, and if sampling is done, an appropriate sampling design is selected. The choice of sampling design depends on the hypotheses to be tested, questions to be answered, or decisions to be made, as well as event-specific information that might indicate appropriate sampling locations. Options include judgmental, targeted, biased, random, and probability-based sampling (see Section D.6).

Probability-based sampling, also called statistical sampling, is appropriate for certain kinds of questions, hypotheses, and decisions. It refers to methods for choosing sample locations so that inference from the results can involve a probability or confidence statement. For example, decision-makers might want to be able to say, "We are $95 \%$ confident that less than $1 \%$ of the floor surface is contaminated (above some specified level)." Or they might want to sample so that there is at least a $99 \%$ probability of discovering a 25-ft2 hot spot, if there is one. Risk-based limits for measurable B. anthracis concentrations on surfaces do not exist at present, but if they were developed in the future for B. anthracis or some other biological warfare agent (BWA), then statistical methods would be appropriate for testing hypotheses such as, "The average concentration of the agent on this surface is below $\mathrm{x}$ " (where $\mathrm{x}$ is a specified number in appropriate units), or "We are $95 \%$ confident that at least $98 \%$ of the surface has a concentration below x."

Probability-based sampling generally includes some form of randomization of sampling locations. However, random sampling is not necessarily statistically designed. If the randomization is not based on a probability model for the outcome, then random sampling is just that, random only. Statistical analyses are possible after the fact for random-only sampling, but a probability model is required to plan ahead for statements of the type described above (i.e., "We are 95\% confident that..."). Closely related to probability-based sampling for statistical inference is sampling for mapping - sampling so that results can be used to create contour plots of contaminant concentration or other, more sophisticated geostatistical analyses.

Three types of probability-based approaches are commonly discussed in the statistical literature pertaining to environmental remediation [Gilbert 1987; Gilbert et. al. 1996; Hardin and Gilbert 1993; U.S. Environmental Protection Agency (EPA) 1996; and EPA 1997). They are:

- Confidence intervals.

- Hypothesis testing.

- Hot spot searching.

\section{E.1 Applicability of Probability-Based Sampling}

In addition to its potential for making decisions with known error probabilities, or estimates with quantitative confidence levels, probability-based sampling has the advantage that it is not susceptible to inadvertent bias in the choice of sampling locations. Because probability-based sampling uses random sampling, it is unlikely that many samples will be collected in atypical locations. It is highly unlikely, for example, that all samples will be collected in areas with the lowest concentrations, thereby failing 


\section{APPENDIX F}

to discover the magnitude of contamination. A sufficient number of randomly located samples will have a distribution of concentrations similar to that of the entire area being sampled, and the results will be representative.

The alternative, judgmental sampling, is highly effective when based on good information and used by individuals with appropriate background and experience. However, if information is incorrect or misinterpreted, judgmental sampling can sample the wrong areas and yield results that fail to show significant contamination, are not representative of the area as a whole, and provide an incorrect basis for subsequent decisions.

In the context of airport cleanup after a BWA attack, probability-based methods are most likely to be appropriate for the assessment of relatively large surface areas where there is little information to indicate where contamination is likely (or unlikely) to be present. The Technical Working Group (TWG) advises the UC on which sampling approach to use in which areas. See Appendix D for additional discussion.

\section{E.2 Probability-Based Sampling for Characterization}

\section{E.2.1 Sampling to Discover Contamination}

One of the primary purposes of characterization environmental sampling is to determine whether or not decontamination is necessary, which depends on whether contamination is present. More generally, the need for decontamination depends on whether contamination is present above some specified level, but at present, the specified level is any level detectable by the sampling methods being used.

In sampling zones where contamination is considered possible but has not been confirmed, and where insufficient information indicates good locations for judgmental sampling, statistical discovery sampling can be used to search for contamination and specify a probability of discovery. This situation could occur in a covert release or after an overt release in zones somewhat distant from the release location. Discovery sampling using random locations is described in more detail in Section E.3.2.

Sampling for hot spots is another form of discovery sampling. In this method, samples are laid out in a regular grid pattern. The grid design and spacing are chosen to have desired detection probability for a given size of hot spot. Hot spot searching could be used to find relatively small areas having higher levels of contamination, which would be cleaned using a surface decontamination method prior to fumigation of the entire zone (see Section E.3.4 and Gilbert 1987).

\section{E.2.2 Sampling to Estimate Maximum Concentration}

If the decontamination design depends on the maximum environmental concentration of the agent, then it is important to estimate the maximum concentration. Locations likely to have the highest concentrations may be predicted by modeling, or event-specific information such as the location of the release (if known) may indicate likely location of the highest concentrations. If such information is unreliable or not available, then the maximum may have to be estimated using sampling alone. Certain sampling designs can optimize the search, such as adaptive cluster sampling (Patil and Rao 1994; Thompson 1992). Such methods are sensitive to spatial variability. The initial sampling must be dense enough to at least come close to the area of maximum concentration. 


\section{APPENDIX E}

\section{E.2.3 Sampling to Create Contaminant Maps}

When contaminant levels are great enough throughout a zone, it is possible to create an iso-contour map of contaminant levels. Mapping methods interpolate between sample locations and require assumptions about how smoothly concentrations vary over the surface. If spatial correlation is assumed and modeled, then it is possible to use statistical methods to optimize the process of reducing the uncertainty in the map's contours. When levels are lower and there are many nondetections, maps may show contours of detectability instead of concentrations. Some of these methods are incorporated in BROOM software.

\section{E.2.4 Sampling Based on Grids}

Sampling designs based on grid patterns provide for relatively even coverage of a surface area (Patil and Rao, 1994). When samples are collected exactly following a grid, they are sometimes called systematic sampling designs (Gilbert 1987). Samples may also be collected at random locations within each grid cell, which is a form a stratified random sampling (Ripley 1987).

\section{E.3 Probability-Based Sampling for Clearance}

The decision to clear an airport for re-use needs to be made with the highest possible level of confidence and the least likelihood of error. Because it can be done so that actual confidence levels are known, and because of its potential for eliminating bias, probability-based sampling can be especially appropriate for clearance sampling.

\section{E.3.1 Clearance Sampling When No Detection is Acceptable}

In the B. anthracis cleanups beginning in 2001, one of the clearance criteria was "no growth of B. anthracis from any clearance sample." This kind of decision rule is sometimes used in industrial quality control under the topic of acceptance sampling (Montgomery, 1997). Two probability-based methods can be used with this kind of decision rule.

\section{E.3.1.1 Discovery Sampling for Clearance}

Discovery sampling refers to sampling strategies in which the EU/TWG specifies a probability with which residual contamination must be found, if it is present anywhere at detectable levels. The approach assumes that residual contamination will be found if at least one clearance sample is placed in a location having residual contamination. It does not mean that all residual contamination will be found, only that at least one sample will be placed in a contaminated location.

The approach is analogous to deciding how hard to look. Collecting more samples corresponds to looking harder. If one looks hard and finds no contamination, then there is some confidence that there is no contamination at detectable levels. If residual contamination is widespread, it is easy to find. The smaller the area of residual contamination, the more one has to look to find it. The important decision is how hard to look. The probability of discovery depends on (1) the percentage of the area that is contaminated at detectable levels, and (2) the number of samples. To design the sampling, the EU/TWG must specify both a desired discovery probability and the percentage of surface area to which that discovery probability applies. It does not matter whether the contamination is located in one contiguous patch or scattered in multiple smaller patches. 


\section{APPENDIXE}

The simplest statistical models for discovery sampling use simple random sampling. If the potential number of samples is huge (as would be the case, for example, when using $100-\mathrm{cm}^{2}$ wipes on a $65,000-\mathrm{ft}^{2}$ floor, for approximately 604,000 potential non-overlapping samples), then the statistical model uses the binomial distribution. If the potential number of samples is not too large (as would be the case, for example, when using $100-\mathrm{ft}^{2}$ vacuum samples on a $65,000-\mathrm{ft}^{2}$ floor, for approximately 650 potential non-overlapping samples, illustrated in Table E-1), then the statistical model uses the hypergeometric distribution.

Table E-1 shows how the number of required samples increases as the size of the area with residual contamination decreases (a range of 20 to $2 \%$ is shown), and as the desired discovery probability increases (a range of 80 to $99 \%$ is shown). The bottom line is that ensuring a high probability for discovering a small amount of residual contamination is expensive.

Table E-1. Example sample numbers for discovery sampling on a $65,000-\mathrm{ft}^{2} \mathrm{floor}$ using $100-\mathrm{ft}^{2}$ vacuum samples ( $n=650$ potential non-overlapping samples).

\begin{tabular}{|l|c|c|c|c|}
\hline \multirow{2}{*}{$\begin{array}{l}\text { Area with residual } \\
\text { contamination }\end{array}$} & $80 \%$ & $90 \%$ & $95 \%$ & $99 \%$ \\
\cline { 2 - 5 } & 8 & 11 & 14 & 21 \\
\hline $20 \%$ & 32 & 45 & 57 & 86 \\
\hline $5 \%$ & 75 & 105 & 133 & 193 \\
\hline $2 \%$ & & & & \\
\hline
\end{tabular}

\section{E.3.1.2 Confirmation Sampling for Clearance}

Confirmation sampling refers to a strategy in which sampling results are evaluated in a two-step process. First, if there are any detections, the success of decontamination is not confirmed. Second, if there are no detections, then an upper confidence limit for the amount of residual contamination is calculated. If the confidence limit is small enough, decontamination is confirmed. The confidence limit depends only on the number of samples, can therefore be specified in advance, and can be set as low as desired.

When the number of potential samples is not too large (as would be the case when using 100- $\mathrm{ft}^{2}$ vacuum samples on a $65,000-\mathrm{ft}^{2}$ floor, for approximately 650 potential non-overlapping samples), then the statistical model uses the hypergeometric distribution, and the upper confidence limit can be set to zero. This leads to a statement of confidence that there is no residual contamination at detectable levels.

Confirmation sampling leads to a much stronger clearance statement than discovery sampling. The hypergeometric case just described leads to an explicit confidence level that there is no residual contamination at detectable levels. Discovery sampling does not demonstrate the absence of contamination, it only states that it was not found.

The confidence level provided by confirmation sampling is based only on the sampling itself and ignores other existing information. Especially in the case of fumigation, other information contributes greatly to confidence in the success of decontamination, namely, the results from fumigation verification sampling (bio-indicator strips). Because clearance sampling does not begin until fumigation has met all of its verification requirements, there is strong prior information indicating that the fumigation was probably successful. Such prior information 


\section{APPENDIX E}

can be incorporated into the confirmation sampling upper confidence limit. Starting with a prior confidence in success, which is based on bio-indicator sampling results and other fumigation process measures, a clearance sampling effort in which contamination is not found increases that confidence.

Confidence in the success of fumigation based on verification parameters is specified in the form of a statistical prior distribution for the amount of residual contamination. In the Table E-2, the prior distribution is summarized in the form of odds of success. For example, 3-to-1 odds of success means that success (no residual contamination) is three times as likely as failure (some residual contamination). With 2-to-1 odds of success, 16 clean samples will increase that to $80 \%$ confidence in success.

Table E-2. Required sample numbers for confirmation sampling.

\begin{tabular}{|l|c|l|l|l|}
\hline & \multicolumn{4}{|c|}{$\begin{array}{c}\text { Desired confidence that there is no } \\
\text { residual contamination }\end{array}$} \\
\hline $\begin{array}{l}\text { Prior odds of } \\
\text { success }\end{array}$ & $80 \%$ & $90 \%$ & $95 \%$ & $99 \%$ \\
\hline No prior & 520 & 585 & 618 & 644 \\
\hline $1: 1$ & 70 & 227 & 390 & 589 \\
\hline $2: 1$ & 16 & 113 & 277 & 551 \\
\hline $3: 1$ & 3 & 47 & 181 & 506 \\
\hline
\end{tabular}

Incorporating prior information greatly reduces the required number of samples (from 585 samples for $90 \%$ confidence with no prior, to 47 samples for $90 \%$ confidence starting with 3:1 odds of success). Nonetheless, the highest level of confidence, $99 \%$, still requires a very large number of samples. The bottom line is that high levels of confidence are expensive.

\section{E.3.2 Sampling Based on Exposure}

The sampling approach, statistical design, and data evaluation can also depend on the nature of the risk and the exposure pathway. For example, if dermal contact to even a small spot of residual contamination may have a serious effect, then discovery, confirmation, or hot spot approaches are probably best. On the other hand, if inhalation of contamination re-aerosolized from a surface is the primary hazard, and it is believed that averaging takes place due to mixing in the air, then statistics based on the average concentration are probably more appropriate.

In the past, the presence of any detectable $B$. anthracis was considered unacceptable regardless of any considerations of exposure pathway or level. Clearance decisions based on averages or other nonzero measures of contamination level were not used. Nonetheless, we include a brief discussion of them.

\section{E.3.2.1 Sampling for the Average}

If a clearance goal specifies a non-zero acceptable level for the average residual contamination, then statistical methods are appropriate. Two approaches can be used. One is to calculate a confidence interval for the average concentration, and insist that the upper confidence limit be below the non-zero acceptable 


\section{APPENDIX F}

level. If the acceptable level is inside the confidence interval then it is uncertain whether the goal has been met, and more sampling will be necessary to tighten the confidence interval. If the entire confidence interval is above the acceptable level, then decontamination has failed. The second approach is to use a formal statistical hypothesis test (see Berthoux and Brown 1994, and many introductory statistics texts).

\section{E.3.2.2 Sampling for Percentiles}

Sampling for percentiles is analogous to sampling for means, except that the acceptable level is specified for an upper percentile of the environmental distribution instead of the average. This leads to confidence intervals known as "tolerance limits."

Upper percentiles are a potential basis for decision criteria because, “... in some situations there may be greater interest in possible acute effects or transient exposures associated with significant short-term risk. Such exposure events may not happen often or on a regular basis" (EPA 1996). This is similar to the exposure model of the hot spot approach, except that with hot spots, the higher levels are assumed to be concentrated in small distinct areas, whereas with percentiles they may be scattered throughout the sampling unit.

\section{E.3.3 Sampling for Hot Spots}

A hot spot is a relatively small contaminated area within a generally clean area. Hot spots can have many different sizes and shapes. Issues include questions such as how much greater than the surrounding area must the level of contamination be within a hot spot to be considered a hot spot, and whether the level of contamination within a hot spot is relatively uniform or varies widely. Several regulatory guidance documents and other publications (EPA 1996, Gilbert 1987, Gilbert and LeGor 1996) define a hot spot as an area that is:

- Contiguous.

- Shaped roughly circularly or elliptically.

- Contaminated in its entirety above a maximum acceptable level.

The shape assumption is necessary to develop a mathematical model for the probability of detection. Because hot spots can have a variety of shapes, the calculated probabilities are approximate. To design a sampling plan to address hot spots, the following criteria must be specified:

- Size, and possibly orientation, of the hot spot.

- Maximum acceptable level.

- Desired probability with which a single hot spot should be discovered.

It is expected that surfaces will be sampled primarily by wipe and vacuum samples. If it is decided to search for hot spots, and very small areas of residual contamination are unacceptable, then the area wiped or vacuumed may not be small relative to the hot spot. This would violate one of the mathematical assumptions of the hot spot methodology. Until risk/exposure criteria and policies have been established, it is unknown whether an effort to correct the model for violations of this assumption would be worthwhile. If a hot spot does not have a well-defined boundary, but gradually tapers off, sampling in the taper region may not detect the hot spot. 


\section{APPENDIX E}

\section{E.3.4 Decision Errors}

There are two types of decision error: false dirty and false clean. A false dirty decision occurs when the decision is made that an area does not meet clearance criteria, when in fact it does. The false clean error is the reverse. Of the two decision errors, the false dirty error will result in additional expenses arising from unnecessary additional decontamination and delay before the site can be returned to productive use. The false clean error is more serious because it puts people at risk. A statistical decision process should follow these steps:

- Assume the sampling unit is still dirty (the conservative assumption and the statistical null hypothesis).

- Collect data using design based on acceptance and decision error rate criteria.

- Reject the assumption only when the data strongly counter the assumption.

This is the "dirty until shown to be clean" approach, which will result in stronger evidence of cleanliness than the "clean until shown to be dirty" approach. In the standard terminology of statistical hypothesistesting theory, the false clean decision is the Type I error (Patil and Rao 1994). In a post-decontamination situation, the "clean until shown to be dirty" approach is equivalent to being overconfident in the effectiveness of the decontamination process.

\section{E.4 Additional Statistical Considerations}

\section{E.4.1 Multiple Statistical Decisions}

The statistical "multiple comparisons" issue needs to be considered. If there are several hundred sampling units in a large site, and each decision is made with, say, a 1\% false clean probability, then the likelihood of there being at least one false clean decision among them all is quite large.

\section{E.4.2 Nondetections}

Nondetections are discussed in some of the cited references. Data analysis that is based on measured concentrations (such as confidence intervals for the average concentration) can be difficult when there are more than a few nondetections among the results. This is likely to be the case after decontamination, and before decontamination in locations not close to the point of release. For this reason, methods based on presence/absence are likely to be easier to use.

A wipe or vacuum sample, especially a large-area vacuum sample, may only partially overlap an area of residual contamination. Depending on the degree of overlap, detection may not occur.

\section{E.5 Literature Review}

The USEPA, National Research Council (NRC), and Department of Energy (DOE) have produced a substantial body of literature regarding post-cleanup clearance sampling in the context of environmental remediation and facility decommissioning. (Terminology in the literature varies. For example, "verification" may be used instead of "clearance.") 


\section{APPENDIX F}

Some additional literature is available in the general area of infection control and environmental control in medical settings. In a medical setting, substantial guidance is available on when, where, how, and how often to clean (or decontaminate). However, little or no guidance specifies how to verify that cleaning procedures have been effective. For example, the CDC Guide on Handwashing and Environmental Control (Garner and Favero 1985) states:

Before 1970, regularly scheduled culturing of the air and environmental surfaces such as floors, walls, and tabletops was widely practiced in U.S. hospitals. By 1970, CDC and the American Hospital Association were advocating that hospitals discontinue routine environmental culturing, since rates of nosocomial infection had not been related to levels of general microbial contamination of air or environmental surfaces, and meaningful standards for permissible levels of microbial contamination of environmental surfaces did not exist (1,2). Between 1970 and 1975, 25\% of U.S. hospitals reduced the extent of such routine environmental culturing (3), and this trend has continued.

In the last several years, there has also been a trend toward reducing routine microbiologic sampling for quality control purposes. In 1982, CDC recommended that the disinfection process for respiratory therapy equipment should not be monitored by routine microbiologic sampling (4). Moreover, the recommendation for microbiologic sampling of infant formulas prepared in the hospital has been removed from this Guideline, since there is no epidemiologic evidence to show that such quality control testing influences the infection rate in hospitals.

CDC guidance does, however, indicate sampling when problems arise:

Microbiologic sampling is indicated during investigation of infection problems if environmental reservoirs are implicated epidemiologically in disease transmission. It is important, however, that such culturing be based on epidemiologic data and follow a written plan that specifies the objects to be sampled and the actions to be taken based on culture results.

The environmental literature, in contrast, contains abundant guidance regarding post-decontamination or post-remediation clearance environmental sampling. The premiere document is the Multi-Agency Radiation Survey and Site Investigation Manual (MARSSIM), developed jointly by the USEPA, DOE, NRC, and DOD (EPA 1997). The purpose of MARSSIM is to provide "detailed guidance for planning, implementing, and evaluating environmental and facility radiological surveys conducted to demonstrate compliance with a dose- or risk- based regulation" (page Roadmap-1). MARSSIM guidance "focuses on the demonstration of compliance during the final status survey following... remedial actions." MARSSIM includes guidance on quality assurance and quality control.

MARSSIM appears to be gaining widespread acceptance as a primary guidance document for such activities. For example, the U.S. Nuclear Regulatory Commission describes MARSSIM as "containing acceptable methods for final status surveys". MARSSIM training is being offered nationwide to the environmental remediation community on an ongoing basis, and it is not unusual for regulatory agencies to ask for a MARSSIM approach to environmental investigations.

Although MARSSIM is intended for radiological contamination, the sampling design and quality assurance/ quality control information is applicable to nonradiological contamination. The MARSSIM chapters that discuss measurement methods and instruments (primarily Chapters 6 and 7 and Appendixes $\mathrm{H}$ and $\mathrm{J}$ ) are 


\section{APPENDIX E}

not applicable to chemical or biological agents, but most of the remainder of MARSSIM is relevant. In fact, MARSSIM could probably serve as the primary guidance document for post-decontamination clearance sampling following a chemical or biological warfare or terrorist event.

MARSSIM recommends rather simple statistical methods, presumably in the hope that they will be adequate in most cases, but it does leave room for the use of more sophisticated methods if they are needed. One approach to clearance sampling not discussed in MARSSIM, and therefore discussed more extensively here, is sampling to find areas that were overlooked by the decontamination process, or so-called hot spots. Another statistical approach not found in MARSSIM but potentially appropriate for BWA is examining the upper percentiles of the contaminant distribution. This approach is discussed in EPA (1996) and Gilbert and LeGor (1996).

\section{E.6 Glossary}

Approval criteria Conditions that a sampling unit must meet to be approved for return to normal use.

Decision error $\quad$ Either of two incorrect decisions: (1) deciding a sampling unit is sufficiently clean when in fact it is not, or (2) deciding a sampling unit is not sufficiently clean when it fact it is.

Detectably clean A level that is considered sufficiently clean, and for which it is highly desirable that a level sampling unit will correctly be declared clean.

Detection probability The probability of detecting residual contamination as large as or larger than the maximum hot spot size.

Distribution

With reference to residual contamination, the range of levels, and the proportions of the sampling unit, that remain contaminated at various levels within that range.

False clean

Deciding a sampling unit is sufficiently clean when in fact it is not. A synonym for false positive.

False dirty Deciding a sampling unit is not sufficiently clean when in fact it is. A synonym for false negative.

False negative Deciding a sampling unit is not sufficiently clean when in fact it is.

False positive Deciding a sampling unit is sufficiently clean when in fact it is not.

Gray region The range of contaminant concentrations between the detectably clean level and the maximum acceptable level.

Grid spacing The distance between grid-based clearances sampling locations. For a square grid, the spacing is the same in both directions.

Hot spot A relatively small contaminated area within a generally clean area. 


\section{APPENDIXE}

Judgmental sampling Subjective selection of sample locations by an individual, preferably based on expert knowledge of the process being studied.

Maximum The highest level that may be considered sufficiently clean. Above this level it is highly

acceptable level

Null hypothesis

desirable that the sampling unit will correctly be declared not clean.

A statistical term that refers to the default assumption made at the beginning of a decisionmaking process.

Percentile A value below which a specified percent of a population lies.

Random sampling Selection of sample locations so that every potential sampling location has an equal chance of being selected.

Residual Contamination left behind by a decontamination process.

contamination

Sampling unit $\quad$ Portion of a site or facility considered as one unit for the purpose of clearance sampling.

\section{E.7 References}

29 CFR 1910.1030 (1999), OSHA Regulations (Standards-29 CFR) Bloodborne Pathogens.

Berthoux, P. M. and L. C. Brown (1994), Statistics for Environmental Engineers, CRC Press Inc., Boca Raton, FL.

Bury, K. V. (1975), Statistical Models in Applied Science, John Wiley \& Sons, New York.

DOE (1997), Draft Handbook for Controlling Release for Reuse or Recycle of Non-Real Property

Containing Residual Radioactive Material, U.S. Department of Energy, Washington, D.C.

EPA (1996), Geostatistical Sampling and Evaluation Guidance for Soils and Solid Media (review draft),

U.S. EPA, Office of Solid Waste.

EPA (1997), Multi-Agency Radiation Survey and Site Investigation Manual (MARSSIM). Washington, DC, U.S. EPA, DOE, NRC, and DOD: NUREG-1575, EPA 402-R-97-016.

Garner, J. S. and M. S. Favero (1985), Guideline for Handwashing and Hospital Environmental Control, U.S. Centers for Disease Control and Prevention, Atlanta, GA.

Gilbert, R. O. (1987), Statistical Methods for Environmental Pollution Monitoring, Van Nostrand Reinhold, New York, NY.

Gilbert, R. O., T. LeGor, et al. (1996), An Overview of Methods for Evaluating the Attainment of Cleanup Standards for Soils, Solid Media, and Groundwater, EPA Volumes 1, 2, and 3, Pacific Northwest National Laboratory: PNL-10908, EC-502.

Hardin, J. W. and R. O. Gilbert (1993), Comparing Statistical Tests for Detecting Soil Contamination Greater than Background, Pacific Northwest Laboratory, Richland, WA, PNL-8989, UC-630.

Montgomery, D. C. (1997), Introduction to Statistical Quality Control, John Wiley \& Sons, New York. NRC (1998), Demonstrating Compliance with the Radiological Criteria for License Termination, U.S. Nuclear Regulatory Commission, Washington, D.C., DG-4006. 


\section{APPENDIXE}

Patil, C. P. and C. R. Rao (1994), Handbook of Statistics Volume 12, Environmental Statistics, Elsevier Science B.V., Amsterdam.

Ripley, B. D. (1980), Spatial Statistics, John Wiley and Sons, New York.

Rutala, W. A. (1996), “APIC Guidelines for Infection Control Practice,” American Journal of Infection Control 24(4), 313-342.

Singer, D. A. (1972), "ELIPGRID: A Fortran IV Program for Calculating the Probability of Success in Locating Elliptical Targets with Square, Rectangular, and Hexagonal Grids," Geocom. Programs 4, 1-16. Singer, D. A. (1975), "Relative Efficiencies of Square and Triangular Grids in the Search for Elliptically Shaped Resource Targets,” J. Res. U.S. Geological Survey 3(2): 163-167.

Thompson, S. K. (1992), Sampling, John Wiley and Sons, New York.

VGPS (1998), Infection Control Literature Review, Victorian State Government Department of Health Services.

VGPS (2000), Cleaning Standards for Victorian Public Hospitals, Victorian Government Publishing Service.

Zirschky, J. and R. O. Gilbert (1984), “Detecting Hot Spots at Hazardous-Waste Sites,” Chem. Engineering 91, 97-100.

Numbered references cited within the Garner and Favero (1985) quotation are as follows:

1. Garner, J. S. and B. P. Simmons (1983), “Guideline for Isolation Precautions in Hospitals,” Infect. Control 4, 245-325.

2. Anderson, R. L., D. C. Mackel, B. S. Stoler, and G. F. Mallison (1982), “Carpeting in Hospitals: An Epidemiological Evaluation," J. Clin. Microbiol. 15, 408-15.

3. American Academy of Pediatrics (AAP) and American College of Obstetricians and Gynecologists (ACOG) (1983), Guidelines for Perinatal Care, Evanston, Illinois, Washington, D.C., AAP, ACOG.

4. Wysowski, D. K., J. W. Flynt, M. Goldfield, et al. (1978), "Epidemic Neonatal Hyperbilirubinemia and Use of a Phenolic Disinfectant Detergent," Pediatrics 61, 165-70. 



\section{APPENDIXF}

\section{Available Decontamination Technologies}

The U.S. Army, the Centers for Disease Control and Prevention (CDC), and other government and privatesector organizations have studied decontamination technology for use against biological warfare agents (BWAs) for decades. Work is currently proceeding at an accelerated pace in view of the $B$. anthracis-laden letters that contaminated numerous buildings in Washington, D.C. and seven states (EPA 2002) in the fall of 2001.

Appendix F describes decontamination technologies most appropriate for use in a large airport that has been intentionally contaminated with $B$. anthracis spores. Information in this appendix draws heavily from what has been learned from decontamination of buildings contaminated with B. anthracis in the fall of 2001. The facilities include the Hart Senate Office Building in Washington, D.C.; the U.S. Capitol's Postal Center (Building 410); Brentwood Mail Distribution and Process Center in Washington, D.C.; Sterling mail facility located in Sterling, Virginia; and other locations. Information is included from the National Response Team's Technical Assistance for Anthrax Response (NRT 2005).

A large airport contains many different types of areas that may need to be decontaminated, ranging from large open atriums and relatively narrow boarding areas to retail concessions. Airports have specialized equipment, such as baggage handlers and industrial air handlers, that can facilitate the spread of spores. Computers, security screening machines, and other complex equipment may need decontamination. Because of such complexity, four types of decontamination technology are required:

- Liquid or semi-liquid reagents to decontaminate exposed porous and nonporous surfaces.

- Gaseous or vaporized reagents to decontaminate difficult-to-reach, porous and nonporous surfaces, including air-handling systems.

- Technologies to decontaminate sensitive electronic equipment.

- Technologies to decontaminate small, personal, or valuable items.

The technologies that were used to remove B. anthracis found in Congressional buildings included: (1) disinfection of hard surfaces with a liquid form of chlorine dioxide, (2) disinfection of hard surfaces with Sandia National Laboratories' (SNL's) Decon Foam 100, (3) high-efficiency particulate air (HEPA) vacuuming on porous surfaces, (4) chamber fumigation of packages and mail with gaseous chlorine dioxide (GAO, 2003), and (5) large-area fumigation of offices with gaseous chlorine dioxide.

Rapid development of new and improved decontamination technologies will ultimately provide airport remediation decision makers with a wide assortment of advanced techniques for specific applications. However, because of the complexity of most civilian scenarios, such as a large airport, it is unlikely that any single decontamination reagent that is applicable to all situations will be found. The most important gap in currently available decontamination technologies is in the area of large, integrated, sensitive electronic equipment.

Two criteria that drive the selection of a decontamination technology for civilian application are toxicity and environmental acceptability of the system used. Both are critical considerations in the enclosed or semienclosed spaces of an airport that require large quantities of decontamination reagent to be dispensed in restricted spaces. 


\section{APPENDIXF}

Existing decontamination reagents are not registered by the U.S. Environmental Protection Agency (EPA) for use against $B$. anthracis contamination. Therefore, for each specific use of a selected reagent to decontaminate a facility contaminated by B. anthracis, a crisis exemption must first be obtained from the EPA. The process can be greatly facilitated by selecting reagents that have been previously registered by the EPA for similar uses (such as a disinfectant or sterilant against spores) or have been granted previous crisis exemptions.

If a release is large, restoring a facility as complex and large as an airport will most likely require the use of multiple contractors for different aspects of cleanup. Table F-1 lists contractors with experience in restoring facilities that have been contaminated with $B$. anthracis. 
Table F-1. Contractors with experience in decontaminating facilities contaminated with B. anthracis.

\begin{tabular}{|c|c|c|}
\hline $\begin{array}{c}\text { Contract } \\
\text { agency }\end{array}$ & Type of contract & Task or role performed \\
\hline \multicolumn{3}{|r|}{ Major Contractors } \\
\hline \multicolumn{3}{|c|}{ IT Corporation (now Shaw Environmental and Infrastructure, Inc.) } \\
\hline EPA & $\begin{array}{l}\text { Conduct removal action } \\
\text { (cleanup) activities at the } \\
\text { Hart Office Bldg. }\end{array}$ & $\begin{array}{l}\text { Prepare buildings for decontamination. Conduct and support decontamination } \\
\text { operations, including fumigation with chlorine dioxide gas. Decontaminate } \\
\text { interior surfaces of buildings, other structures, cars, and other vessels. Provide } \\
\text { for collection, containment, transportation, and disposal of contaminated } \\
\text { materials from site operations. Support EPA sampling teams and other federal } \\
\text { responders, including response technicians, to assist with decontamination } \\
\text { activities. }\end{array}$ \\
\hline \multicolumn{3}{|c|}{ Shaw Environmental and Infrastructure, Inc. } \\
\hline $\begin{array}{l}\text { U.S. Dept } \\
\text { of State }\end{array}$ & $\begin{array}{l}\text { Conduct removal action } \\
\text { (cleanup) activities at the } \\
\text { Sterling Facility. }\end{array}$ & Prime contractor, formerly the IT Group of Monroeville, PA. \\
\hline \multicolumn{3}{|c|}{ U.S. Army Corps of Engineers Rapid Response Program } \\
\hline $\begin{array}{l}\text { U.S. Dept } \\
\text { of State }\end{array}$ & $\begin{array}{l}\text { Conduct removal action } \\
\text { (cleanup) activities at the } \\
\text { Sterling Facility. }\end{array}$ & Project management. \\
\hline \multicolumn{3}{|c|}{ Sabre Oxidation Technologies, Inc. } \\
\hline EPA & $\begin{array}{l}\text { Provide technical support } \\
\text { to cleanup activities at Hart } \\
\text { Office Bldg.a Fumigation of } \\
\text { Brentwood, Hamilton, and } \\
\text { AMI Facilities. }\end{array}$ & $\begin{array}{l}\text { Provide engineering support during assessment of the feasibility and design } \\
\text { of systems for fumigating air-handling return system in the Daschle suite. } \\
\text { Fumigation of Brentwood, Hamilton, and AMI Facilities using chlorine dioxide. }\end{array}$ \\
\hline \multicolumn{3}{|c|}{ Strategic Technology Enterprises, Inc. } \\
\hline $\begin{array}{l}\text { U.S. Dept } \\
\text { of State }\end{array}$ & $\begin{array}{l}\text { Conduct removal action } \\
\text { (cleanup) activities at the } \\
\text { Sterling Facility. }\end{array}$ & Fumigation of Sterling facility using vaporous hydrogen peroxide. \\
\hline \multicolumn{3}{|c|}{ Tetra Tech EM, Inc. } \\
\hline EPA & $\begin{array}{l}\text { Provide technical support } \\
\text { to cleanup activities at Hart } \\
\text { Office Bldg. }\end{array}$ & $\begin{array}{l}\text { Provide on-scene coordinator and incident commander fumigation design } \\
\text { procedures, including details on fumigant delivery; concentration; operating } \\
\text { conditions, such as temperature and humidity; fumigant containment and } \\
\text { recovery; and monitoring of parameters. Provide detailed design for delivering } \\
\text { fumigant, equipment requirements and specifications, flow schematics, } \\
\text { and schedules and operating procedures during fumigation. Provide } \mathrm{ClO}_{2} \\
\text { specialist to help EPA oversee fumigation setup. Technical support to on-scene } \\
\text { coordinator in developing chronology of events, including researching files to } \\
\text { develop a comprehensive report. Monitor and assist with oversight of chlorine } \\
\text { dioxide fumigation. Assist with health and safety at the site, conduct sampling, } \\
\text { assist and oversee off-gassing, inventory, and return treated items. } \\
\text { Support on-scene coordinator in presentations and briefings on post-treatment } \\
\text { and design of chlorine dioxide use in HVAC system. Sample critical items } \\
\text { (plastic, leather, and polyester); determine how ethylene oxide and its } \\
\text { derivatives are maintained in the materials and off-gas over time. }\end{array}$ \\
\hline
\end{tabular}




\section{APPENDIX F}

\begin{tabular}{|c|c|c|}
\hline $\begin{array}{l}\text { Contract } \\
\text { agency }\end{array}$ & Type of contract & Task or role performed \\
\hline \multicolumn{3}{|c|}{ Earth Tech, Inc. } \\
\hline EPA & $\begin{array}{l}\text { Conduct removal action } \\
\text { (cleanup) activities at the } \\
\text { Hart Office Bldg. }{ }^{\text {a }}\end{array}$ & $\begin{array}{l}\text { Provide decontamination services and other direct support to sampling teams. } \\
\text { Decontaminate interior surfaces of buildings, other structures, and interior } \\
\text { and exterior surfaces of cars and other vessels identified by the on-scene } \\
\text { coordinator. Collect all expended cleaning agents and materials for treatment } \\
\text { and/or disposal. } \\
\text { Provide decontamination facilities and services for response personnel and their } \\
\text { equipment. Inventory items - segregating clean and contaminated materials and } \\
\text { salvageable and expendable items - and provide documentation of inventoried } \\
\text { items. } \\
\text { Propose a decontamination strategy for critical items (including personal items, } \\
\text { such as photographs, framed diplomas, and equipment). Decontaminate critical } \\
\text { and salvageable items from the Capitol Complex, including setting up work } \\
\text { zones for items to be decontaminated and for personnel decontamination. } \\
\text { Return property after decontamination. } \\
\text { Provide contamination reduction and isolation facilities and operations that } \\
\text { improve and ensure safe access to contaminated areas and items and prevent } \\
\text { further spread of contamination. }\end{array}$ \\
\hline \multicolumn{3}{|c|}{ Environmental Quality Management, Inc. } \\
\hline EPA & $\begin{array}{l}\text { Conduct removal action } \\
\text { (cleanup) activities at the } \\
\text { Hart Office Bldg. }{ }^{\text {a }}\end{array}$ & $\begin{array}{l}\text { Provide personnel and equipment, including portable decontamination facility. } \\
\text { Collect expended cleaning agents and materials for treatment and/or disposal. } \\
\text { Dispose of materials or items that could not be decontaminated. }\end{array}$ \\
\hline \multicolumn{3}{|r|}{ Supporting Contractors } \\
\hline \multicolumn{3}{|c|}{ CDM Federal Programs Corporation } \\
\hline EPA & $\begin{array}{l}\text { Provide general support to } \\
\text { cleanup activities at the Hart } \\
\text { Office Bldg. }{ }^{\text {a }}\end{array}$ & $\begin{array}{l}\text { Oversee preparation, handling, placement, and collection of spore strips used } \\
\text { during fumigation with chlorine dioxide gas and ethylene oxide gas. Develop } \\
\text { a procedure for spore strip emplacement; removal; and critical item tagging, } \\
\text { tracking, and shipping. } \\
\text { Provide sampling, such as swipe and high-efficiency particulate arresting } \\
\text { (HEPA) vacuum (including efforts to collect, prepare, and ship samples), item } \\
\text { decontamination, and minor remediation work. } \\
\text { Support critical item degassing in Beltsville, MD. Maintain critical item } \\
\text { inventories; coordinate release/return of critical items to congressional staffers. } \\
\text { Support } \mathrm{ClO}_{2} \text { decontamination of congressional mail packages. }\end{array}$ \\
\hline \multicolumn{3}{|c|}{ Roy F. Weston, Inc. } \\
\hline EPA & $\begin{array}{l}\text { Provide technical support to } \\
\text { cleanup activities at the Hart } \\
\text { Office Bldg. }\end{array}$ & $\begin{array}{l}\text { Develop documents and plans used during response activities (e.g., standard } \\
\text { operating procedures for sampling, decontamination, and source reduction). } \\
\text { Provide reconnaissance, photo documentation, and sampling of congressional } \\
\text { office buildings. } \\
\text { Provide technical support to select and implement decontamination procedures; } \\
\text { building-specific plan development for B. anthracis remediation, including } \\
\text { sampling plans, isolation plans, decontamination plans, and item recovery } \\
\text { plans; and sampling support for B. anthracis analysis using HEPA and wipe } \\
\text { sampling techniques; perform oversight of removal crews. } \\
\text { Provide swab and HEPA sampling and decontamination support. Provide bag- } \\
\text { and-tag operations of critical and salvageable items in congressional office } \\
\text { buildings. Provide air monitoring during chlorine dioxide fumigation. }\end{array}$ \\
\hline
\end{tabular}




\begin{tabular}{|c|c|c|}
\hline $\begin{array}{l}\text { Contract } \\
\text { agency }\end{array}$ & Type of contract & Task or role performed \\
\hline \multicolumn{3}{|c|}{ Ecology \& Environment, Inc. } \\
\hline EPA & $\begin{array}{l}\text { Provide technical support to } \\
\text { cleanup activities at the Hart } \\
\text { Office Bldg. }\end{array}$ & $\begin{array}{l}\text { Develop sampling and decontamination plans, sample labels, chain of } \\
\text { custodies, and maps to support sampling activities and track sampling results. } \\
\text { Perform sampling, monitoring, and decontamination of areas in the Capitol } \\
\text { Hill complex. Conduct sampling tracking and handling activities, including } \\
\text { preparing samples for shipping. } \\
\text { Compile and review background data and organize site documentation files. } \\
\text { Provide technical support to the operations section and support to the EPA } \\
\text { Mobile Lab. }\end{array}$ \\
\hline \multicolumn{3}{|c|}{ Lockheed-Martin } \\
\hline EPA & $\begin{array}{l}\text { Provide general support to } \\
\text { cleanup activities at the Hart } \\
\text { Office Bldg. }\end{array}$ & $\begin{array}{l}\text { Assist in monitoring temperature and relative humidity inside office buildings } \\
\text { and in monitoring chlorine dioxide, chlorine, wind speed and direction, } \\
\text { temperature, and relative humidity in surrounding area. } \\
\text { Assist in developing and evaluating B. anthracis fumigation procedures using } \\
\text { spore strips in a test facility; training other contractors in handling and placing } \\
\text { spore strips at the office building. } \\
\text { Provide ambient air monitoring for chlorine dioxide using tape meters and a } \\
\text { portable meteorological tower to document that no chlorine dioxide is emitted } \\
\text { from treatment area. Onsite assistance to ensure that spore strip sampling is } \\
\text { conducted properly and that data management is accurate and complete. }\end{array}$ \\
\hline \multicolumn{3}{|c|}{ Guardian Environmental Services, Inc. } \\
\hline EPA & $\begin{array}{l}\text { Conduct removal action } \\
\text { (cleanup) activities at the } \\
\text { Hart office Bldg. }\end{array}$ & $\begin{array}{l}\text { Assist in removing items from contaminated office suites in the congressional } \\
\text { office buildings, including removal of contaminated office furniture, office } \\
\text { equipment, and carpet. Construct isolation chambers, decontamination } \\
\text { chambers, and related structures. }\end{array}$ \\
\hline \multicolumn{3}{|c|}{ URS Operating Services, Inc. } \\
\hline EPA & $\begin{array}{l}\text { Provide technical support at } \\
\text { the Hart Office Bldg. }{ }^{a}\end{array}$ & Provide sampling for B. anthracis in the Capitol Hill complex. \\
\hline \multicolumn{3}{|c|}{ MVM Security \& Staffing Services } \\
\hline EPA & $\begin{array}{l}\text { Provide security support to } \\
\text { cleanup activities at the Hart } \\
\text { Office Bldg. }{ }^{\text {a }}\end{array}$ & $\begin{array}{l}\text { Provide security personnel to staff the single entrance/exit and to patrol offices } \\
\text { during cleanup to ensure no unauthorized personnel enter work area and assure } \\
\text { that property items are not removed from the work area without EPA approval. }\end{array}$ \\
\hline \multicolumn{3}{|l|}{ TSI, Inc. } \\
\hline EPA & $\begin{array}{l}\text { Provide supplies at the Hart } \\
\text { Office Bldg. }\end{array}$ & Provide Porta Count plus respirator fit tester. \\
\hline \multicolumn{3}{|c|}{ Kemron Environmental Services, Inc. } \\
\hline EPA & $\begin{array}{l}\text { Provide technical support to } \\
\text { cleanup activities at the Hart } \\
\text { Office Bldg. }{ }^{\text {a }}\end{array}$ & $\begin{array}{l}\text { Perform air sampling and HEPA vacuuming services. } \\
\text { Remove critical items and documents, spray affected areas with chlorine } \\
\text { dioxide, and perform cleaning and breakdown of work zones. } \\
\text { Assist EPA in evaluating possible remediation of the heating, ventilation, and } \\
\text { air-conditioning (HVAC) system, including evaluation of affected areas, and } \\
\text { construction of critical barriers inside ductwork to isolate affected areas from } \\
\text { uncontaminated areas. After HVAC fumigation, provide confirmatory sampling } \\
\text { support, interior duct sampling, additional cleaning of system (including post- } \\
\text { fumigation scrub down inside the ducts), and removal of duct insulation. }\end{array}$ \\
\hline
\end{tabular}




\begin{tabular}{|c|c|c|}
\hline $\begin{array}{l}\text { Contract } \\
\text { agency }\end{array}$ & Type of contract & Task or role performed \\
\hline \multicolumn{3}{|c|}{ HMHTTC Response Team, Inc. } \\
\hline EPA & $\begin{array}{l}\text { Conduct removal action } \\
\text { (cleanup) activities at the } \\
\text { Hart Office Bldg. }\end{array}$ & $\begin{array}{l}\text { Perform cleanup activities, including construction and removal of isolation } \\
\text { barriers, HEPA vacuuming, and application of liquid chlorine dioxide. } \\
\text { Provide 24-hour support for decontamination and rescue operations at the } \\
\text { Capitol Hill B. anthracis site. }\end{array}$ \\
\hline \multicolumn{3}{|c|}{ Southwest Research Institute } \\
\hline EPA & $\begin{array}{l}\text { Provide laboratory support to } \\
\text { cleanup activities at the Hart } \\
\text { Office Bldg. }{ }^{\text {a }}\end{array}$ & $\begin{array}{l}\text { Provide analysis of spore strips placed in various locations during cleanup } \\
\text { operations. Receive and perform daily observations of thousands of spore strips. }\end{array}$ \\
\hline \multicolumn{3}{|c|}{ University of California, Berkeley, Sponsored Projects Office } \\
\hline EPA & $\begin{array}{l}\text { Provide technical support to } \\
\text { cleanup activities at the Hart } \\
\text { Office Bldg. }{ }^{\text {a }}\end{array}$ & $\begin{array}{l}\text { Participate in and support program plan development relating to spore } \\
\text { sterilization technologies for remediation of federal facilities. } \\
\text { Develop experimental and field test plans and methodologies for } \\
\text { characterization/modeling spore killing processes and kinetics and factors that } \\
\text { affect the efficacy of spore killing in field-scale applications. } \\
\text { Establish lab systems for measuring gas-phase sporicidal effects at office } \\
\text { and mail facilities. Provide lab analytical support for measuring gas-phase } \\
\text { sporicidal effects. Develop experimental and test plans and methodologies for } \\
\text { assessing and validating spore killing processes. } \\
\text { Determine concentrations of chlorine dioxide needed to decontaminate B. } \\
\text { anthracis on Capitol Hill. Prepare } 31,500 \text { test strips containing a bacillus similar } \\
\text { to B. anthracis and send to Capitol Hill. Exposed strips sent to labs; results then } \\
\text { sent to the UC, Berkeley, to be included in a consolidated final report. }\end{array}$ \\
\hline \multicolumn{3}{|c|}{ Silva Consulting Services, LLC } \\
\hline EPA & $\begin{array}{l}\text { Provide technical support to } \\
\text { cleanup activities at the Hart } \\
\text { Office Bldg. }{ }^{\text {a }}\end{array}$ & $\begin{array}{l}\text { Maintain sample-management system software in a private, secure environment } \\
\text { on the Internet. Provide EPA and designated contractor personnel secure, } \\
\text { controlled access to database. This system could generate a large variety of } \\
\text { reports to address particular questions about sampling results. }\end{array}$ \\
\hline \multicolumn{3}{|c|}{ Science Applications International Corp } \\
\hline EPA & $\begin{array}{l}\text { Provide technical support to } \\
\text { cleanup activities at the Hart } \\
\text { Office Bldg. }{ }^{\text {a }}\end{array}$ & $\begin{array}{l}\text { Provide consulting services to the EPA on-scene coordinator in environmental } \\
\text { remediation of } B \text {. anthracis-contaminated buildings at Capitol Hill. Support } \\
\text { data interpretation of spore strips used to test the efficacy of the kill of } B \text {. } \\
\text { anthracis, data validation, review of documents, assistance in document } \\
\text { preparation, and report writing. Coordinate efforts with University of } \\
\text { California, Berkeley. }\end{array}$ \\
\hline \multicolumn{3}{|c|}{ Biomarine, Inc. } \\
\hline EPA & $\begin{array}{l}\text { Provide supplies for cleanup } \\
\text { activities at the Hart Office } \\
\text { Bldg. }\end{array}$ & $\begin{array}{l}\text { Provide equipment, including biopaks, facemasks, oxygen cylinders, gel tubes, } \\
\text { foam scrubbers, coolant canister foam, flow restrictors, and biopak service and } \\
\text { retrofit kits. }\end{array}$ \\
\hline \multicolumn{3}{|c|}{ Envirofoam Technologies, Inc. } \\
\hline EPA & $\begin{array}{l}\text { Provide supplies at the Hart } \\
\text { Office Bldg. }{ }^{\text {a }}\end{array}$ & Provide Sandia Decon Foam and backpack dispensing units. \\
\hline \multicolumn{3}{|c|}{ Safeware, Inc. } \\
\hline EPA & $\begin{array}{l}\text { Provide supplies at the Hart } \\
\text { Office Bldg. }{ }^{\mathrm{a}}\end{array}$ & Provide respirators with battery and cartridge. \\
\hline
\end{tabular}




\begin{tabular}{|c|c|c|}
\hline $\begin{array}{l}\text { Contract } \\
\text { agency }\end{array}$ & Type of contract & Task or role performed \\
\hline \multicolumn{3}{|c|}{ Airgas Safety } \\
\hline EPA & $\begin{array}{l}\text { Provide supplies at the Hart } \\
\text { Office Bldg. }{ }^{a}\end{array}$ & Provide air-purifying respirators. \\
\hline \multicolumn{3}{|c|}{ U.S. Art Company, Inc. } \\
\hline EPA & $\begin{array}{l}\text { Provide technical support at } \\
\text { the Hart Office Bldg. }{ }^{a}\end{array}$ & $\begin{array}{l}\text { Provide training on procedures for handling, packaging, and decontaminating } \\
\text { artifacts (paintings, sculptures, and other art forms) from the Hart Building. }\end{array}$ \\
\hline \multicolumn{3}{|c|}{ Mine Safety Appliances } \\
\hline EPA & $\begin{array}{l}\text { Provide supplies at the Hart } \\
\text { Office Bldg. }{ }^{\mathrm{a}}\end{array}$ & Provide self-contained breathing apparatus system. \\
\hline \multicolumn{3}{|c|}{ Coastal Safety \& Health Services, Inc. } \\
\hline EPA & $\begin{array}{l}\text { Provide supplies at the Hart } \\
\text { Office Bldg. }{ }^{\text {a }}\end{array}$ & Provide indoor air-quality meter. \\
\hline \multicolumn{3}{|c|}{ New Horizons Diagnostics Corp } \\
\hline EPA & $\begin{array}{l}\text { Provide supplies for cleanup } \\
\text { at the Hart Office Bldg. }{ }^{\text {. }}\end{array}$ & Provide B. anthracis-detection kits. \\
\hline \multicolumn{3}{|c|}{ Armed Forces Radiobiology Research Institute (AFRRI) } \\
\hline $\begin{array}{l}\text { U.S. Postal } \\
\text { Service }\end{array}$ & $\begin{array}{l}\text { Provide technical support at } \\
\text { Brentwood. }\end{array}$ & $\begin{array}{l}\text { Provide independent microbiology assessment of } \mathrm{ClO}_{2} \text { fumigation efficacy; } \\
\text { laboratory analysis of biological indicator spore strips; HEPA vacuum and } \\
\text { swipe sample analysis for viable B. anthracis spores; and radiation sterilization } \\
\text { of personal and high-value items. }\end{array}$ \\
\hline
\end{tabular}

${ }^{\mathrm{a}}$ GAO Report on the EPA (see GAO 2003).

${ }^{\mathrm{b}} \mathrm{http}: / /$ www.loudoun.gov/general/anthraxfaqs.htm\#gen

\section{F.1 Preparing for Decontamination}

B. anthracis decontamination should be conducted only after characterization sampling has been properly completed and a determination has been made that spore contamination exists at one or more specific locations at the airport. Characterization activities are discussed in Section 2 of the main text. The range of activities associated with remediation and leading up to the implementation of decontamination itself is discussed in Section 3 of the main text. Other steps, such as the necessity of obtaining a crisis exemption from the EPA before using a decontamination reagent against $B$. anthracis, are explained in this appendix.

\section{F.1.1 Source Reduction}

The objective of source reduction is to decrease the amount of contamination in a facility before the main decontamination activity. In the Sterling mail facility, decision makers decided to remove and dispose of all porous materials, such as carpets, furniture, and drywall partitions as well as most nonporous items, such as mail- and parcel-sorting machines. This decision may have been motivated in part by plans to reconfigure the building after decontamination. Items that could be damaged by the cleaning process or by extensive handling, such as computers, computer parts, and telephones, were removed and properly disposed. Some materials taken out of the building were subjected to a dilute bleach solution $(0.5$ percent, $\mathrm{pH} 7$ sodium hypochlorite) or treated with Spor-Klenz RTU (a USEPA-registered biocide) prior to removal and disposal. 


\section{APPENDIXF}

Spor-Klenz RTU is a peracetic-acid/hydrogen-peroxide-based biocide used by the pharmaceutical industry as part of the cleaning and decontamination regimen in clean rooms and biological containment laboratories (see section F.3.1).

At the Sterling mail facility, Capitol Hill B. anthracis site, Department of State mail facility, Department of Justice mail facility, and NBC, essential items were sent to an offsite ethylene oxide sterilization chamber and then returned for reuse. Such chambers have been demonstrated to be effective and preservative of most materials. Items sent for such treatment were treated with Spor-Klenz, but were not pretreated with bleach because chlorine in the bleach can react with ethylene oxide and produce toxic byproducts.

A survey conducted in a representative airport identified a wide variety of substances and materials that would potentially require decontamination. One approach for many substrates, such as carpet, chairs, partitions, acoustic ceiling tile, waste containers, and benches, is to remove and properly dispose of them, then replace them with new ones. At AMI, all these items were left in place and decontaminated without noticeable damage after 9 months. Thus, decisions on removal versus replacement should be made on a case-by-case basis and in view of the choice of decontamination agent. Important considerations are that removing items prior to decontamination requires workers to use high-level PPE, and any removed material not subsequently decontaminated must be transported and disposed of as infectious medical waste, adding to cost. Materials decontaminated in place may be reused or disposed of as municipal waste. Municipal-waste site operators may be reluctant to take material from a BWA incident even after it has been decontaminated; thus, pre-established disposal plans are essential. A disposal plan should reflect any state, local, or facility requirements (e.g., specific decontamination actions, post-decontamination sampling, and PPE for transportation and disposal facility workers) for disposal of decontaminated material as municipal waste.

\section{F.2 Containment and Isolation Technologies}

Containment of a contaminated area is required to prevent the spread of a contaminant by movement of workers, equipment, and air. Isolation of a contaminated area refers to sealing a site to permit fumigation and prevent release of fumigant. The extent of isolation and type of containment technologies used will depend on factors such as the size of the affected area, types of surfaces involved, and extent of contamination. For example, containment procedures can involve closing all fire doors and HVAC registers and sealing them with sealant. Larger areas can be closed off and isolated by constructing barriers using 2 by $4 \mathrm{~s}$, plastic sheeting, duct tape, and other products, and sealing all openings to the outside.

During decontamination of the Hart Senate Office Building, construction and removal of containment barriers were conducted by HMHTTC Response Team, Inc. At the Sterling Facility, the entire building underwent an extensive sealing process to prevent escape of particulates and gas from the facility into the atmosphere. The Sterling Facility was sealed from both the interior and exterior. On the inside, visible cracks in floors, walls, and ceilings were sealed with expanding foam sealant or silicone caulking; all floor drains were sealed; and windows on outside walls were covered. Seals were checked weekly during cleanup activities. On the outside, all visible cracks in walls were sealed with foam sealant or caulking; all skylights and other openings in the roof were covered, sealed, and insulated with poly sheeting and foil tape. Truck dock areas were framed and covered with poly sheeting; and all roof leaks were sealed.

Recently, materials normally used for tenting houses and buildings during insecticidal fumigation have been used for chlorine dioxide fumigations. The materials are economical and readily available. Special fabrics designed by the Department of Defense (DOD) for CBW agent protection could also be considered for use 


\section{APPENDIX F}

in containment and isolation. The fabrics were designed to be effective against CBW agents; are strong, durable, and lightweight; can be welded or sealed; and are resistant to decontamination reagents. However, they can be costly and could have a long acquisition lead time. Thus, if they are used, consideration should be given to advance purchase and stockpiling.

A greater level of containment and isolation can be achieved by creating negative air pressure to prevent the outward flow of air. This can be done using portable, HEPA-filtered, negative air units (NAUs). If fumigation is to be conducted, such units can be used to prevent escape of the fumigant, and they can be fitted with carbon canisters or other filtration devices that can break down the fumigant. NAUs can consist of a fan and HEPA filter alone for containment, or for isolation of fumigant, they can include elements such as a chemical scrubber, demister, carbon bed, and stack. Air within the building is exhausted through HEPA filters at a rate sufficient to pull a slightly negative pressure in the zone with contamination. NAUs are used during any fumigation and their installation in areas that most likely require fumigation (based on initial data) allows for an additional measure of protection. NAUs can move from 2,000 to 30,000 cubic feet of air per minute, and the number of NAUs required will depend on the volume of the decontamination zone. A sufficient number of NAUs to create a negative pressure of from -0.03 to -0.005 inches of water is required. Multiple NAUs may be necessary, depending on the capacity of the NAUs and size of the airport.

\section{F.3 Surface Decontamination Technologies}

Technologies for surface decontamination are the best understood of all the different types of decontamination technologies. Surface decontamination can be used as the primary decontamination technology (as in the case of a low-grade preparation of spore material that is not readily aerosolized), or as a means of source reduction prior to fumigation (in the case of high-grade materials that can be readily aerosolized).

During response to B. anthracis contamination of the Hart Office Building, the EPA developed protocols for using aqueous-based, oxidizing, surface decontamination reagents, such as liquid chlorine dioxide, bleach, hydrogen peroxide, and peracetic acid. The protocols are good, general guides for most aqueous-based, surface decontamination reagents. All oxidizers should be used on hard surfaces only. For porous surfaces, HEPA vacuum filtration can be used for source reduction, but porous materials are generally removed unless fumigation is used. Application of aqueous-based oxidizers must be conducted under a crisis exemption issued by the EPA specific to the buildings or treatment sites identified under the crises exemption. Application must be conducted according to use instructions by the responsible cleanup personnel who follow an event-specific Remediation Action Plan. Such a plan includes the following items:

- Sampling to determine the extent of spore contamination at specific locations.

- Spot decontamination (source reduction) of highly contaminated surfaces through HEPA filter vacuuming.

- Gross surface decontamination with liquid decontamination reagent.

- Post-treatment sampling to determine that B. anthracis decontamination has been effective.

- Re-treatment if growth is detected on any collected sample.

These steps apply to facilities where treated surfaces will be reused or the facility will be reoccupied. The steps do not necessarily apply to wastes or debris intended for disposal in an appropriate disposal facility. 


\section{APPENDIXF}

\section{F.3.1 Available Surface Decontamination Reagents}

Both the DOD and the Department of Energy (DOE) have invested heavily in the area of surface decontamination reagents, and they both have developed mature technologies. During 2003, the DOE program was transferred to the Department of Homeland Security (DHS), which continues research in this arena. Although the U.S. Army has several other available surface decontamination reagents, they are not suitable for civilian settings because of their caustic or toxic nature, and they are also most suitable for reasonably nonporous surfaces.

Surface-acting reagents are sufficient to decontaminate sites with limited, surficial B. anthracis contamination when applied to hard, nonporous surfaces. At such sites, porous materials are generally removed as part of the source-reduction process. Vacuum decontamination (see Section F.3.3) is useful for highly porous surfaces in a civilian setting. At sites where fumigations are performed, pre-treatment with surface-acting agents may be part of the source-reduction process. Table F-2 lists the aqueous-based, surface decontamination reagents currently available that are most appropriate for use in a large-scale civilian setting, such as an airport.

Table F-2. Aqueous-based, exposed-surface decontamination reagents.

\begin{tabular}{|c|c|}
\hline Decontamination reagent & Description \\
\hline \multicolumn{2}{|c|}{ Previous EPA registration and crisis exemption issued for inactivation of B. anthracis spores } \\
\hline Chlorine dioxide & $\begin{array}{l}\text { Products containing sodium chlorite or stabilized chlorine } \\
\text { dioxide are usually mixed with another reactive chemical- } \\
\text { usually an acid - to produce chlorine dioxide in a liquid state. }\end{array}$ \\
\hline Hydrogen peroxide & Marketed as Virex STF. A 3-25\% liquid sterilizes at $20^{\circ} \mathrm{C}$. \\
\hline Peroxyacetic acid & $\begin{array}{l}\text { A } 0.2 \% \text { solution provides antiseptic ability. A } 2-5 \% \text { solution } \\
\text { provides sterilization at } 20^{\circ} \mathrm{C} \text {. }\end{array}$ \\
\hline $\begin{array}{l}\text { Peroxyacetic acid/hydrogen } \\
\text { peroxide-based sterilant }\end{array}$ & $\begin{array}{l}\text { A peracetic acid/hydrogen peroxide-based sterilant is used } \\
\text { by the pharmaceutical industry as part of the cleaning and } \\
\text { decontamination regimen in clean rooms and biological } \\
\text { containment labs. Marketed as Oxonia Active, KX-6049, } \\
\text { Actril Cold Sterilant, and Spor-Klenz RTU. A liquid sterilant/ } \\
\text { disinfectant. }\end{array}$ \\
\hline $\begin{array}{l}\text { 10:1 diluted, } \mathrm{pH} 7 \text { sodium } \\
\text { hypochlorite }(5,250-6,000 \mathrm{ppm})\end{array}$ & $\begin{array}{l}\text { Commercially available, } 2 \text { to } 5 \% \text {, aqueous solution of sodium } \\
\text { hypochlorite. }\end{array}$ \\
\hline \multicolumn{2}{|c|}{ No previous EPA registration or crisis exemption issued for inactications of B. anthracis spores } \\
\hline Calcium hypochlorite & $\begin{array}{l}\text { High-test hypochlorite (HTH) is a } 65 \text { to } 70 \% \text { aqueous } \\
\text { solution of calcium hypochlorite used in swimming pools. }\end{array}$ \\
\hline $\begin{array}{l}\text { SNL Decon Foam } \\
\text { DF } 200\end{array}$ & $\begin{array}{l}\text { Foam that contains both hydroperoxide and hydroperoxy- } \\
\text { carbonate anions in a formulation that contains a surfactant, } \\
\text { fatty alcohols, and water-soluble polymers to enhance the } \\
\text { physical stability of the foam. }\end{array}$ \\
\hline L-Gel & $\begin{array}{l}\text { Gel formulation that is based on the active ingredient Oxone } \\
\text { (potassium peroxymonosulfate manufactured by Dupont) and } \\
\text { a fumed silica gelling agent ( } 15 \text { to } 20 \% \text { Cabosil EH-5), which } \\
\text { produces extended contact time on contaminated surfaces. }\end{array}$ \\
\hline Virkon S & $\begin{array}{l}\text { 1\% peroxymonosulfate solution. Marketed as a high-level } \\
\text { disinfectant (1\%). Higher concentrations can be sporicidal. }\end{array}$ \\
\hline
\end{tabular}




\section{APPENDIXF}

In the event of a terrorist attack involving BW agents, the need for immediate acquisition of sufficient decontamination reagent becomes critical. The availability of such material can depend on the time of year for certain reagents. Limitations in availability can be overcome by stockpiling and storing selected decontamination reagents (such as general, broad-based decontamination reagents). Stockpiling would allow for immediate access to surface decontamination reagents when initiating decontamination operations.

\section{F.3.1.1 Liquid Chlorine Dioxide}

Information on the use of liquid chlorine dioxide for B. anthracis decontamination can be found at http://www.epa.gov/pesticides/factsheets/chemicals/chlorinedioxidefactsheet.htm and is summarized here. Liquid chlorine dioxide formulations were first registered in the 1960s as disinfectants and are used in a variety of ways, including on pets and farm animals; in bottling plants; and in food processing, handling, and storage plants. Pesticide products containing either sodium chlorite or stabilized chlorine dioxide are usually mixed with another "reactive" chemical — usually an acid — to produce chlorine dioxide in a liquid or gaseous state. Liquid chlorine dioxide is then applied to hard surfaces with a sponge or mop, or as a coarse spray. The potency of a chlorine dioxide solution depends on the concentration of gas dissolved in solution. A gentle application with the least amount of gas volatilization is preferred, such as wiping by hand or spraying with low pressure and maximum droplet size. To aid in gas retention, sodium chlorite or Triton DF 12 may be added to the formulation.

The EPA granted a crisis exemption for the use of liquid chlorine dioxide in B. anthracis decontamination. Under the crisis exemption, registered products containing sodium chlorite may be sold or distributed only to employees of Federal, state, or local government agencies, or employees of the U.S. Postal Service, for B. anthracis cleanup.

Gross surface decontamination using a liquid solution of chlorine dioxide is conducted under the following conditions:

- A rate of $500 \mathrm{mg} / \mathrm{L}$ liquid chlorine dioxide is applied. A solution generated onsite should be used immediately.

- Applications are made at room temperature $\left(68^{\circ} \mathrm{F}\right.$, or $\left.20^{\circ} \mathrm{C}\right)$.

- Treatment area must remain dark because ultraviolet light increases the rate of chlorine dioxide decay and renders it less potent.

- Treatments must have a wet contact time of at least $30 \mathrm{~min}$. The surface must remain wet through the entire contact time, and multiple applications may be necessary.

- The surface is allowed to air dry and is not wiped. The treated area may have a nontoxic, fine residue.

Any remaining liquid chlorine dioxide must be removed from treated areas of the building before people are allowed to re-enter. During the Hart Office Building response, HMHTTC Response Team, Inc., was responsible for applying liquid chlorine dioxide. IT Corporation also was involved in decontaminating interior surfaces of buildings, other structures, cars, and other vessels. Kemron Environmental Services, Inc., removed critical items, sprayed affected areas with chlorine dioxide, and performed cleaning and breakdown of work zones. Chlorine solution was also used to clean hotspots at the Sterling Facility. 


\section{APPENDIXF}

\section{F.3.1.2 Hydrogen Peroxide and Peroxyacetic Acid}

Liquid hydrogen peroxide and peroxyacetic acid in B. anthracis decontamination is discussed in the National Response Team's report (NRT 2005), and further information can be found at: http://www.epa. gov/pesticides/factsheets/chemicals/hydrogenperoxide_peroxyaceticacid_factsheet.htm. These substances are known as peroxy compounds. Both are oxidizing agents and are registered as sterilants capable of killing spores. Hydrogen peroxide is widely used as a disinfectant because of its reactive properties. In the home, hydrogen peroxide can be found in diluted form (3\% to $10 \%)$, whereas industrial uses involve concentrated solutions (30\% or greater). Diluted forms of hydrogen peroxide are often used as cleansers for human cuts and scrapes. Formulated as a liquid, peroxyacetic acid is used as a disinfectant and sanitizer, and is usually applied as a spray or as a mop-on solution.

The EPA issued crisis exemptions for the limited sale, distribution, and use on hard (nonporous) surfaces of four registered products containing both hydrogen peroxide and peroxyacetic acid. These products for use against $B$. anthracis spores are: Oxonia Active (EPA Registration Number 1677-129), KX-6049 (EPA Registration Number 1677-158), Actril Cold Sterilant (EPA Registration Number 52252-7), and SporKlenz Ready-to-Use (EPA Registration Number 52252-7-1043). Spor-Klenz RTU is a biocide used by the pharmaceutical industry as part of the cleaning and decontamination regimen in clean rooms and biological containment labs. The EPA also issued a crisis exemption for the product Virex STF, which contains only hydrogen peroxide. Applications of these pesticide products under the crisis exemption are limited to specific buildings or treatment sites identified by the EPA or other federal, state, or local authorities. Gross surface decontamination using liquid solutions is conducted under the following conditions.

Conditions of application for Oxonia Active and KX-6049:

- A rate of 5,000 parts per million (ppm) peroxyacetic acid is applied; this rate is achieved by adding $10 \mathrm{oz}$ Oxonia Active to 1 gal water, or $13 \mathrm{oz} \mathrm{KX}-6049$ to 1 gal water.

- Applications are made at room temperature $\left(68^{\circ} \mathrm{F}\right.$, or $\left.20^{\circ} \mathrm{C}\right)$.

- Treatments must have a wet contact time of at least 20 minutes.

Conditions of application for Actril Cold Sterilant and Spor-Klenz Ready-to-Use:

- Undiluted product is applied.

- Applications are made at room temperature $\left(68^{\circ} \mathrm{F}\right.$, or $\left.20^{\circ} \mathrm{C}\right)$.

- Treatments must have a wet contact time of at least 10 minutes.

Conditions of application for Virex STF:

- Undiluted product is applied.

- Applications are made at room temperature $\left(68^{\circ} \mathrm{F}\right.$, or $\left.20^{\circ} \mathrm{C}\right)$.

- Treatments must have a wet contact time of at least 15 minutes.

\section{F.3.1.3 Sodium hypochlorite}

Information on the use of bleach for B. anthracis decontamination can be found at http://www.epa.gov/pesticides/ factsheets/chemicals/bleachfactsheet.htm and is summarized here. Registered liquid bleach products contain sodium hypochlorite, a compound used as a cleaner and to kill bacteria, fungi, and viruses. Sodium hypochlorite 
is a strong oxidizing agent. The U.S. Air Force explored the use of a bleach solution against Bacillus subtilis, var. niger, spores in seeking an effective biological decontaminant for large-scale decontamination. The active ingredient in the reagent was $1.25 \%$ sodium hypochlorite. This concentration destroyed $99.9 \%$ of bacterial contaminant present and was reported to be equally effective at a $\mathrm{pH}$ of 10 . Although this may sound effective, a $99.9 \%$ kill is a reduction of only 3 logs. If the goal were to inactivate visible powder that contained $10^{8}$ viable spores, a 3-log reduction would leave $10^{5}$ viable spores. Product efficacy depends on specific conditions, including temperature $\left(68^{\circ} \mathrm{F}\right), \mathrm{pH}(7)$, organic load (low), concentration $(5,250$ to $6,000 \mathrm{ppm})$ and wet contact time (60 $\mathrm{min})$.

The EPA issued a crisis exemption for the limited sale, distribution, and use of EPA-registered bleach products for use against $B$. anthracis. Under the crisis exemption, only registered bleach products may be sold or distributed to employees of the EPA, other Federal, state, or local government agencies, and the U.S. Postal Service for use in B. anthracis decontamination. Gross surface decontamination using bleach is conducted under the following conditions:

- A bleach solution close to, but not above, pH 7 (neutral) and 5,250 to 6,000 parts per million (ppm) is prepared by mixing one part bleach $(5.25$ to $6.00 \%)$ to one part white vinegar to eight parts water. Bleach and vinegar must not be combined together directly; rather, some water must first be added to the bleach (e.g., two cups water to one cup of bleach), then vinegar (e.g., one cup), and then the rest of the water (e.g., six cups). The $\mathrm{pH}$ of the solution should be tested with a paper test strip.

- Treated surfaces must remain in contact with the bleach solution for 60 minutes. Repeated applications are necessary to keep surfaces wet.

- Product can be removed by wiping the treated area with sterilized cloth.

The product can cause significant collateral damage, such as corrosion. Sodium hypochlorite is prepared in several concentrations including 17.5, 13.2, 7.0, and 5.0\%, and it is available in 5-, 15-, and 55-gal drums. Two of the numerous manufacturers of this product are Surpass Chemical Co., Inc., Brookfield, Wisconsin (518/434-8101), and the Hydrite Chemical Co., Albany, New York (414/792-1450). Both manufacturers supply sodium hypochlorite in 12.5 to $15 \%$ concentrations in 53-gal drums at an approximate cost of $\$ 1.25 / \mathrm{gal}$.

\section{F.3.1.4 Calcium Hypochlorite}

Calcium hypochlorite is used as a disinfectant in swimming pools, ponds, drinking water, and other water or wastewater systems. Although it is registered as a pesticide by the EPA, it was not used in the recent B. anthracis responses, and thus was not granted a crisis exemption. Calcium hypochlorite (HTH) is an excellent and effective decontamination agent for a broad range of biological agents and is applicable to both chemical warfare agents (CWAs) and biological warfare agents (BWAs). The long-term storage stability of hypochlorites is not good, and such reagents would have to be monitored periodically for their total, active chlorine content or be replaced periodically.

The manufacturers of HTH appear to be limited in number and consist of the Pittsburgh Plate Glass Co., the Olin Corp., and Biolab Distributors. HTH is shipped and stored in plastic-lined drums in quantities of 100, 25, and $9 \mathrm{lb}$. Although calcium hypochlorite is a powerful oxidizing agent, it is considered to be safe and stable if stored in a cool, dry location. The cost of calcium hypochlorite is approximately $\$ 1.00$ to $\$ 1.10$ per $1 b$, or approximately $\$ 110.00$ per 100 -lb drum. Distributors indicate that they normally have about eighty 100-1b drums in inventory during the winter months, and approximately 350 drums during summer months. 


\section{APPENDIXF}

\section{F.3.1.5 DF 200}

Decon Foam (DF 200) was developed by Sandia National Laboratories (SNL) through the Department of Energy's Chemical and Biological National Security Program, which has subsequently become part of the Department of Homeland Security. DF 200 reactive foam is effective against both CWAs and BWAs, was developed to be noncorrosive (or much less corrosive than the hypochlorites), is nontoxic, and is applicable to both hard and porous surfaces. Efforts are currently being directed toward optimizing the foam formulation, performing live-agent testing on various substrates, developing better deployment systems for the foam concentrate, and conducting additional biological agent screening. The foam is currently being manufactured and distributed by Modec, Inc.

DF 100 had a previous crisis exemption for use on B. anthracis decontamination, but the exemption was revoked because it failed to pass the AOAC Sporicidal Activity Test (SAT) when the EPA tested it. After the product was reformulated as DF 200, the product passed the AOAC SAT for hard, nonporous surfaces, but requires a 3-hour contact time.

This nontoxic reagent is reported to be available as a foam, fog, or solution to decontaminate CWAs or BWAs, as well as sensitive equipment. The sensitive equipment capability is possible because the delivery system can produce fine particles (7 to 15 microns) producing 200 to $600 \mathrm{ppm}$ of vapor, rather than an aqueous solution. The reagent is available as a backpack system, delivering up to 5 gal of reagent (the equivalent of 250 foam gal). A skid-mounted assembly is capable of delivering several hundreds of gallons of foam per minute for large-area decontamination. The unit is supplied with the necessary spray units, nozzles, containment tanks, and personal protection gear. The unit is available in two separate drums from Modec, Inc., 4725 Oakland St., Denver, Colorado, 80238 (800-967-7887). Information on the product is available at www.deconsolutions.com. Prices range from $\$ 500$ to $\$ 17,000$ for delivery systems (hand-held fogger to ATV-mounted unit). Concentrate for foam runs in the range of $\$ 50$ gal. SFO has outfitted a bus for emergency decontamination that uses DF 200.

\section{F.3.1.6 L-Gel}

Under the DOE and DHS program, Lawrence Livermore National Laboratory (LLNL) has developed a gel-based decontamination reagent, called L-Gel, which is specifically designed to adhere to high, vertical surfaces. L-Gel contains Oxone ${ }^{\mathrm{TM}}$ (a $0.5-$ to $0.8-\mathrm{N}$ solution of potassium peroxymonosulfate) as the active ingredient (manufactured by Dupont) along with a fumed-silica gelling agent (15 to 20\% Cabosil EH-5), which is compatible with strong oxidizing agents and produces extended contact time of the gel on a contaminated surface. A wet contact time of one hour is required.

The product has been shown to be effective against a series of chemical and biological surrogates, on a variety of substrates, and in live-agent testing. L-Gel is fast acting, effective on most substrates, and thixotropic (it clings to walls and surfaces). It is characterized as noncorrosive (or much less corrosive than most other reagents), nontoxic, and its use results in only nonhazardous residual byproducts. Outdoor use requires no cleanup, but the dried residue indoors may need to be vacuumed and discarded.

Hand application can be used for small areas where contaminant reduction is required. Small, handheld or backpack applicators are sufficient to apply the reagent to medium-size areas requiring only local decontamination. However, this equipment should not be used when contamination by high-grade, aerosolizable spores is suspected because of the likelihood of spreading the contamination. Equipment appropriate for small-scale decontamination includes small, hand-held devices, similar to fire extinguishers. 


\section{APPENDIX F}

L-Gel has good shelf life and is relatively inexpensive $\left(\sim \$ 1.00 / \mathrm{m}^{2}\right)$. L-Gel is currently undergoing commercialization but is available from LLNL in the event of a national emergency. No previous crisis exemption has been granted for use of L-Gel against BWAs.

\section{F.3.1.7 Virkon $S$}

Virkon $\mathrm{S}$ is a 1\% peroxymonosulfate solution that is marketed as a high-level disinfectant (1\%). Greater concentrations can be sporicidal. The product has little or no human or ecological toxicity and is biodegradable and nonflammable. However, its relatively low $\mathrm{pH}$ (2.6) could be problematic in some applications, and its potential reactivity to certain substrates is a definite drawback. Cost is a little higher than other peroxy-related products. Virkon S has been seen to decontaminate various forms of bacteria, viruses, and fungal species. Testing at LLNL showed it not to be effective on CWAs, but it was highly effective against BWAs. Decontamination seems to be slower and less effective than other related peroxycontaining products. A disadvantage is that the product comes as powder, which must be mixed thoroughly with water. Another drawback is that the oxidizer can be rapidly destroyed by very low organic loads.

\section{F.3.2 Summary of Application Data}

Table F-3 summarizes application data, and Table F-4 summarizes compatibility data on the various surface decontamination reagents.

Table F-3. Application rates and contact times for surface decontamination reagents.

\begin{tabular}{|c|c|c|c|c|}
\hline $\begin{array}{c}\text { Decontamination } \\
\text { reagent }\end{array}$ & Solution concentration & $\begin{array}{c}\text { Application } \\
\text { considerations }\end{array}$ & $\begin{array}{c}\text { Contact } \\
\text { time }(\mathrm{min})\end{array}$ & Cleanup \\
\hline $\begin{array}{l}\text { Liquid chlorine } \\
\text { dioxide }\end{array}$ & $\begin{array}{l}500 \text { ppm made at site from } \\
\text { precursors }\end{array}$ & $\begin{array}{l}\text { Apply at rate sufficient to } \\
\text { wet surface in a dark room at } \\
68^{\circ} \mathrm{F} \text {. }\end{array}$ & 30 & Air dry, no wiping \\
\hline $\begin{array}{l}\text { Oxonia Active and } \\
\text { KX-6049 }\end{array}$ & $\begin{array}{l}5000 \text { ppm diluted at site from } \\
\text { concentrate }\end{array}$ & $\begin{array}{l}\text { Preclean surface. } \\
\text { Room temp } 68^{\circ} \mathrm{F} \text {. }\end{array}$ & 20 & Air dry \\
\hline Sodium hypochlorite & $\begin{array}{l}5250-6000 \text { ppm made by } \\
\text { mixing } 1 \text { part bleach }(5.25- \\
6.0 \%) \text { to } 1 \text { part white vinegar to } \\
8 \text { parts water }\end{array}$ & $\begin{array}{l}\text { Apply at } \mathrm{pH} 7 \text { with room } \\
\text { temp } 68^{\circ} \mathrm{F} \text {. Surface should } \\
\text { have low organic load. }\end{array}$ & 60 & $\begin{array}{l}\text { Wipe treated area } \\
\text { with sterile cloth }\end{array}$ \\
\hline L-Gel & $\begin{array}{l}\text { Gel is stirred to liquefy } \\
\text { (use mixer on end of drill) }\end{array}$ & $\begin{array}{l}\text { Apply at rate to completely } \\
\text { cover surface. Surface should } \\
\text { have low organic load. }\end{array}$ & 60 & $\begin{array}{l}\text { Vacuum residue as } \\
\text { needed }\end{array}$ \\
\hline Virkon S & As purchased (1\%) & $\begin{array}{l}\text { Surface should have low } \\
\text { organic load. }\end{array}$ & 60 & \\
\hline
\end{tabular}




\section{APPENDIXF}

Table F-4. Compatibility data.

\begin{tabular}{|l|c|c|c|}
\hline \multicolumn{1}{|c|}{ Decontamination reagent } & Relative toxicity & $\begin{array}{c}\text { Relative } \\
\text { corrosivity }\end{array}$ & $\begin{array}{c}\text { Relative } \\
\text { flammability }\end{array}$ \\
\hline Chlorine dioxide & High & Mid & High \\
\hline Hydrogen peroxide/peracetic acid & Mid to high & Mid & Mid to high \\
\hline Sodium hypochlorite & High & High & Mid to high \\
\hline HTH & High & High & Mid to high \\
\hline DF 200C & Low & Mid & Mid \\
\hline L-Gel & Low & Mid & Mid \\
\hline Virkon S & Low & Mid & Mid \\
\hline
\end{tabular}

\section{F.3.3 High-Efficiency Particulate Air Vacuum Technology}

High-efficiency particulate air (HEPA) vacuuming has primarily been used for sampling and source reduction, but it could potentially be used as a decontamination method as well. It is especially useful for porous materials. In addition, it removes dirt and other debris that may reduce the effectiveness of subsequent decontamination, either by using surface reagents or fumigation. An advantage of this technology is that there is little potential for collateral damage. However, the technology can only be used to remove surface contamination (and thus may not be appropriate for the internal workings of sensitive electronic equipment). In addition, there is potential for spreading contamination if the exhaust is allowed to stir the air in the contaminated area.

The use of HEPA vacuuming is discussed in the National Response Team's Technical Assistance for Anthrax Response (NRT 2005) and is summarized here. The HEPA filter vacuum may be used on porous and nonporous surfaces and applied to collect spores from a "top-to-bottom" or "cold-to-hot" approach. A variety of vacuum assemblies may be needed for the various surfaces and shapes to be treated. The HEPA vacuum is systematically applied to collect spores from the area of least contamination to the area of greatest contamination, and from the highest to lowest elevation. The HEPA filter sock-fitted vacuum is applied to the contaminated area beginning with the outer edge of the contaminated area, working inward. Vacuuming of all surfaces should be conducted at an extremely slow and controlled rate to minimize dispersion of potentially contaminated dust during the vacuuming process. Work should also progress from ceiling to floor. After vacuuming, the area may be cleaned using surface decontamination reagent and sampled to verify decontamination or to determine the extent of remaining contamination.

\section{F.3.3.1 Past Uses and Vendors}

HEPA vacuuming was used for sampling and source reduction in offices in Washington, DC, and in numerous U.S. Postal Service facility decontamination projects. Kemron Environmental Services, Inc., provided the EPA with HEPA vacuuming services at the Capitol Hill site. HMHTTC Response Team, Inc., also conducted HEPA vacuuming operations at the site. The U.S. Government does not endorse any vendor or recommend one vendor over another. 


\section{APPENDIX F}

\section{F.3.4 Choice of Surface Decontamination}

The choice of a surface decontamination technology depends on the surface material and the grade and quantity of contamination. For porous surfaces, HEPA vacuuming is the only potential surface decontamination technology, although its use has not been validated. For hard surfaces, HEPA vacuuming can be followed with liquid oxidizing reagents. Reagents that have received previous crisis exemptions should be given preference. Although bleach is the most readily available of the liquid reagents, it also has the greatest potential for collateral damage. Hydrogen peroxide and peroxyacetic acid can be purchased ready to use, although large quantities may require some lead time. Liquid chlorine dioxide must typically be made onsite. The choice of surface decontamination reagents will depend on availability and acceptability of potential collateral damage.

\section{F.4 Large-Area-Decontamination Fumigation Reagents}

Both vaporous hydrogen peroxide and chlorine dioxide have been used in B. anthracis decontamination activities. This section discusses their use in wide-area decontamination. When using any gaseous decontamination reagent, the concept of the CT value is important. The CT value represents the product of concentration $(C)$ of fumigant and the length of time $(T)$ the fumigant is kept at that concentration. When the product of concentration and time is constant theoretically, so is the biological effect over a limited range of concentration and time. For very short or long exposures, the biological effect may vary. For example, the CT of $1 \mathrm{mg}$ of vaporous hydrogen peroxide per liter of air held for $4 \mathrm{hr}$ is $4 \mathrm{mg}-\mathrm{hr} / \mathrm{L}$. This is the same as $0.5 \mathrm{mg}$ of vaporous hydrogen peroxide per liter of air held for $8 \mathrm{hr}$, that is, $4 \mathrm{mg}-\mathrm{hr} / \mathrm{L}$. Thus, the tradeoff between time and concentration is readily apparent. Even though oxidizers can take a long time to act, and large-scale use can be logistically complex, their safety profile is a benefit when performing large-area fumigation.

\section{F.4.1 Vaporous Hydrogen Peroxide}

The liquid form of hydrogen peroxide is a well-known oxidizing agent. Dilute solutions (3 to 10\%) are commonly used in household products as a cleanser for cuts and scrapes. Hydrogen peroxide decomposes into water and oxygen.

Solutions of $30 \%$ or greater are used by the pulp and textile industries, as well as in environmental applications. Vaporous hydrogen peroxide is used as an antimicrobial pesticide for decontaminating sealed enclosures, such as scientific workstations, isolators, pass-through rooms, medical and diagnostic devices, and for other biological safety applications. Vaporous hydrogen peroxide has been used for years in the pharmaceutical industry on electronic equipment because of its favorable compatibility profile. It is penetrative in smaller pieces of equipment, but its penetration into large pieces of sensitive equipment is unknown.

Vaporous hydrogen peroxide is registered by the EPA to kill bacterial spores on environmental surfaces in an enclosed area. However, vaporous hydrogen peroxide is not registered for use on B. anthracis contamination in buildings. Therefore, for each specific use of vaporous hydrogen peroxide to decontaminate buildings for B. anthracis, a crisis exemption must be obtained from the EPA (see Section F.7).

The EPA has issued two crises exemptions for vaporous hydrogen peroxide to date. The first crises exemption was issued for use by the General Services Administration and its contractors for B. anthracis 


\section{APPENDIXF}

decontamination at Building 410, which is located in the Anacosta Navy Yard, Washington, DC. The second crisis exemption was issued for the Department of State and its contractors for use on the Sterling mail facility, Building SA-42, located in Sterling, Virginia. Because this process could only be used for spaces up to 250,000 cubic feet, the facilities had to be divided into zones that were fumigated and then cleared one at a time.

Vaporous hydrogen peroxide is produced using an aqueous solution of $35 \%$ hydrogen peroxide. This solution is introduced into a vaporous hydrogen peroxide generator, where it is flash vaporized. The vapor is then introduced into a facility through piping from the generator either directly into a facility or into the facility's HVAC ductwork. The target CT is $216 \mathrm{ppm}$ for $4 \mathrm{hr}$. Galvanized ductwork causes rapid breakdown of vaporous hydrogen peroxide, making it difficult to obtain the desired CT. However, even after replacement of galvanized ductwork with HDPE, decomposition made it difficult to reach the target CT.

A large-capacity, proprietary, vaporous hydrogen peroxide generator manufactured by STERIS Corporation is currently the only available technology to produce large quantities of hydrogen peroxide vapor. The highcapacity system can decontaminate spaces of between 100,000 and 250,000 $\mathrm{ft}^{3}$. Decontamination zones with large volumes would need to be subdivided into smaller areas. However, multiple generators were used by STERIS with the U.S. Department of Defense to increase treatment volumes. Because humidity greatly affects the sterilization process (high humidity causes hydrogen peroxide vapor to condense out), each zone is dehumidified prior to sterilization. Current applications of the large-scale system use a separate air handling system stationed outside the impacted area to control humidity and air flow. Upon completion of sterilization, the building air from inactive zones is evacuated through an exhaust module. The exhaust module consists of a bank of HEPA filters, a catalytic bed to convert any exhausted hydrogen peroxide vapor into water vapor and oxygen, and a blower. The speed of the blowers is modulated to ensure that air flow out of the building via the exhaust always exceeds that of air flow entering the impacted area, resulting in a negative pressure within the impacted area. Removal of vaporous hydrogen peroxide is done by generator module and natural decay, which is the major factor.

\section{F.4.1.1 Past Uses and Vendors}

The 1.4-million- $\mathrm{ft}^{3}$ Building 410 was subdivided into zones of 250,000 $\mathrm{ft}^{3}$. The Sterling mail facility was initially subdivided into seven zones of $200,000 \mathrm{ft}^{3}$, and subsequently into ten zones ranging from about 40,000 to 200,000 $\mathrm{ft}^{3}$. The U.S. Army Corps of Engineers' (USACE) Rapid Response Program was commissioned by the Department of State to decontaminate the Sterling Facility. USACE awarded a contract to Shaw Environmental and Infrastructure, Inc. (formerly the IT Group of Monroeville, PA). Fumigation was conducted by Strategic Technology Enterprises, Inc., a subsidiary of STERIS Corporation. The U.S. Government does not endorse any vendor or recommend one vendor over another.

\section{F.4.2 Chlorine Dioxide}

Information on the use of gaseous chlorine dioxide in B. anthracis decontamination can be found at the following internet address:

http://www.epa.gov/pesticides/factsheets/chemicals/chlorinedioxidefactsheet.htm and is summarized here. Chlorine dioxide $\left(\mathrm{ClO}_{2}\right)$ is an antimicrobial pesticide recognized since the early 1900s for its disinfectant properties. Chlorine dioxide kills microorganisms by disrupting transport of nutrients across the cell wall. Chlorine dioxide can be generated in a gas or liquid form and smells like 


\section{APPENDIXF}

chlorine bleach. Chlorine dioxide is created using pesticide products containing either sodium chlorite or stabilized chlorine dioxide that are usually mixed with another reactive chemical — usually an acid — to produce chlorine dioxide in a liquid or gaseous state.

Chlorine dioxide gas is generated onsite from a liquid phase and is released through stripping the gas from the liquid into a sealed treatment area, where it remains for several hours before being removed. It can be used on porous and nonporous surfaces. The treated area must be completely sealed and a negative air unit used to maintain negative pressure. After treatment is completed, chlorine dioxide gas is neutralized with sodium bisulfite or exhausted through a bank of carbon filters. The treatment may leave a fine residue, but it is not toxic.

Chlorine dioxide should not be confused with chlorine gas. They are two distinct chemicals that react differently and produce byproducts that have little in common. Chlorine dioxide breaks down into less-toxic chloride ions, so the residual safety risk is greatly reduced. In 1988, the EPA registered chlorine dioxide gas as a sterilant. The EPA first registered chlorine dioxide gas as an antimicrobial pesticide in the 1980s. Chlorine dioxide gas is registered for sterilizing manufacturing and laboratory equipment, environmental surfaces, tools, and clean rooms. It is also used in pharmaceutical research and production. However, chlorine dioxide gas is not registered for use on $B$. anthracis contamination in buildings. Therefore, for each specific use of chlorine dioxide gas to decontaminate buildings for B. anthracis, a crisis exemption must be obtained from the EPA (see Section F.7). Crisis exemptions for gaseous chlorine dioxide (from certain sodium chlorite products) were issued for use at the Hart Senate Office Building (November 30, 2001), to decontaminate the exterior of mail packages that had been received by U.S. Government offices (February 26, 2002), to test fumigation of lockers in a trailer at the Brentwood Mail Distribution and Process Center, Washington, DC (June 21, 2002), at the AMI Building in Boca Raton FL, and at the USPS Distribution and Process Center at Hamilton, NJ.

The crisis exemptions for gaseous chlorine dioxide issued for the Hart Senate Office Building and for the exterior of mail packages involved products containing sodium chlorite as the active ingredient to generate gaseous chlorine dioxide onsite, followed by clearance environmental sampling to confirm that treated areas were free from B. anthracis spores (e.g., showed no growth when samples were cultured in the laboratory). Conditions of application were as follows:

- A minimum concentration of 500 to $550 \mathrm{ppm}$ chlorine dioxide gas was initially applied for a minimum of 12 hours, for a minimum total of 6,000 ppm-hr. Later, the concentration was increased to $750 \mathrm{ppm}$ for a total of 9,000 ppm-hr.

- Applications were made at a minimum temperature of $75^{\circ} \mathrm{F}$.

- Relative humidity was maintained at a minimum of $75 \%$.

This process does not have a limitation on the volume of space that can be treated, so fumigation of large volumes can be conducted without secondary separations. All openings are sealed with thermal foam, and windows are covered to eliminate UV decay of chlorine dioxide. Temperature and relative humidity are adjusted to and maintained at $75^{\circ} \mathrm{F}$ and $75 \%$, respectively. The existing HVAC system can be used to deliver the chlorine dioxide gas. A supplemental delivery system can also be used, if necessary. A concentration of $750 \mathrm{ppm}$ is maintained for $12 \mathrm{hr}$, resulting in a CT of $9000 \mathrm{ppm}-\mathrm{hrs}$. The liquid precursors are staged outside the facility and are mixed with water within a generator. The water with dissolved chlorine dioxide gas is then pumped to emitters within the area to be decontaminated. $\mathrm{ClO}_{2}$ is converted into the gaseous form when stripped through the emitters. The gas has a visible yellow-green color and a distinctive smell. The gas dissipates quickly in open areas and in sunlight. 


\section{APPENDIXF}

\section{F.4.2.1 Past Uses and Vendors}

Chlorine dioxide gas was used for decontaminating parts of the Hart Office Building. This technology was also used at the Brentwood Mail Distribution and Process Center and the Trenton Mail facility. The EPA oversaw chlorine dioxide fumigation at the Hart Office Building (GAO 2003). The IT Corporation (now known as Shaw Environmental and Infrastructure) supported fumigation activities, and Sabre Inc. conducted the fumigation. In the Hart Office Building, about 100,000 $\mathrm{ft}^{3}$ were decontaminated, 5.8 million $\mathrm{ft}^{3}$ were decontaminated at the Trenton Mail Facility, and 14 million $\mathrm{ft}^{3}$ were decontaminated at the Brentwood Mail Distribution and Process Center. The fumigation and delivery system used in Washington was transported to Trenton. As part of the consortium of BioONE, Sabre also recently completed fumigation of the B. anthracis-contaminated AMI building in Boca Raton, Florida. Sabre acted as the engineering and fumigation contractor during the fumigation and conducted all fumigation verification and clearance activities. The U.S. Government does not endorse any vendor or recommend one vendor over another. Lockheed-Martin has assisted in monitoring temperature and relative humidity inside office buildings and in monitoring chlorine dioxide, chlorine, wind speed and direction, temperature, and relative humidity in surrounding areas during fumigation. They also provided ambient air monitoring for chlorine dioxide using tape meters and a portable meteorological tower to document that no chlorine dioxide was being emitted from the treatment area.

Kemron Environmental Services, Inc., sprayed affected areas with chlorine dioxide and performed cleaning and breakdown of work zones. They assisted the EPA in evaluating possible decontamination of the HVAC systems, including evaluation of affected areas and construction of critical barriers inside the ductwork to isolate affected areas from uncontaminated areas. After fumigation of the affected HVAC systems, Kemron provided confirmatory sampling support, interior duct sampling, additional system cleaning (including postfumigation scrub-down inside the ducts), and removal of duct insulation.

\section{F.4.3 Paraformaldehyde}

Paraformaldehyde should be included for consideration as a potential fumigant in future fumigation activities. Paraformaldehyde has a long history of use to decontaminate biosafety hoods and laboratories in clinical and research settings, and it was used to decontaminate entire buildings from the former bioweapons program at Fort Detrick. Following the 2001 attacks, it was used to decontaminated mail machines in a small fumigation in the Department of Justice mail facility and to decontaminate 47,000 diplomatic mail pouches at the Department of State mail facility prior to remediation of that facility. The set-up time for fumigations with paraformaldehyde can be short, and the key process variable requirements are well worked out. Formaldehyde is a probable human carcinogen and an animal carcinogen following chronic exposures, but long-term exposure would not be an issue following fumigations. Where time is of the essence in returning facilities to productive reuse, paraformaldehyde deserves serious consideration.

\section{F.4.4 Verification of the Effectiveness of the Fumigation Process}

The effectiveness of the fumigation process is verified by two methods: (1) process monitoring (temperature, relative humidity, fumigant concentration, and contact time throughout fumigation), and (2) use of biological indicators (BIs). Monitoring temperature, relative humidity, fumigant concentration, and contact time requires locating stations of sensors throughout the area to be fumigated and wiring them to access the data during the fumigation process. Wireless technology is available for temperature and relative humidity probes. 


\section{APPENDIX F}

Monitoring fumigant concentration for vaporous hydrogen peroxide can be done using commercially available $\mathrm{H}_{2} \mathrm{O}_{2}$ sensors employing electrochemical sensing elements or IR spectroscopy, which can provide real-time measurement of the vapor concentration. No real-time sensors are currently available to monitor chlorine dioxide concentration. As a result, such monitoring requires a complex system of sampling tubing throughout a facility, from which air samples can be collected for subsequent wet chemistry analysis.

BIs, also known as spore strips or spore discs, are used as part of process to confirm the effectiveness of fumigation. The BIs consist of a known number of surrogate microorganisms (typically $10^{6}$ spores) affixed to filter paper strips or discs of stainless steel placed inside a tyvex bag. The surrogate is related to B. anthracis but is not pathogenic to humans. The selected surrogate is known to be resistant to the mode of sterilization and is at least as resistant as the target agent. Geobacillus stearothermophilus endospores are the common surrogates used in vaporous hydrogen peroxide fumigation; Bacillus atropheus endospores are used in chlorine dioxide fumigation. Subsequent growth or failure of the microorganisms to grow under suitable conditions indicates the failure or adequacy of fumigation, respectively.

Previously, the number of BIs required has been determined on the basis of floor space of the areas being decontaminated (one BI per $100 \mathrm{ft}^{2}$ of floor space). Locations for BIs are selected to provide a complete distribution throughout the spaces being treated. In previous vaporous hydrogen peroxide fumigations, no growth on all BIs was required for fumigation verification. Any BI growth necessitated refumigation. In previous chlorine dioxide fumigations, positive BIs did not necessarily result in refumigation; instead, areas with positive BIs received additional scrutiny during clearance sampling.

\section{F.4.4.1 Past Uses and Vendors}

During cleanup operations at the Hart Office Building, Southwest Research Institute provided analysis of spore strips placed in various locations. Southwest personnel received samples and performed daily observations on thousands of spore strips.

CDM Federal Programs Corporation oversaw preparation, handling, placement, and collection of spore strips used during fumigation with chlorine dioxide gas and ethylene oxide gas. CDM personnel developed a procedure for spore strip emplacement, removal, and shipping.

Lockheed-Martin assisted in monitoring temperature and relative humidity inside office buildings and in monitoring chlorine dioxide, chlorine, wind speed and direction, temperature, and relative humidity in surrounding areas during fumigation. They assisted in developing and evaluating B. anthracis fumigation procedures using spore strips in a test facility, and they trained other contractors in the handling and placement of spore strips in the office building. The U.S. Government does not endorse any vendor or recommend one vendor over another.

\section{F.4.4.2 Ambient Air Monitoring}

The use of gaseous fumigants requires monitoring of ambient air to ensure that the fumigant does not escape a facility in concentrations that may be a hazard to the surrounding population. As part of the crisis exemption application, the EPA requires a written Ambient Air Monitoring Plan. A mobile EPA Trace Atmospheric Gas Analyzer (TAGA) bus has been an integral part of ensuring the safety of fumigation in previous decontamination efforts (Hart Building, Brentwood, Trenton, and AMI). The bus is equipped with a quadrapole mass spectrometer to analyze ambient air sampled by a pump as the bus drives around the perimeter of a facility. 


\section{APPENDIXF}

For ambient air monitoring after using vaporous hydrogen peroxide, sentry monitors, such Dräger or other commercially available $\mathrm{H}_{2} \mathrm{O}_{2}$ sensors, are located around the facility during the decontamination process. The monitors can be installed to provide real-time data back to a data logging system. Hand-held, realtime monitors and colorimetric tubes can be used to investigate unacceptable levels of vaporous hydrogen peroxide by the sentry system.

Chlorine dioxide can be monitored using the mobile EPA TAGA bus; vaporous hydrogen peroxide can be monitored using a sentry system. If a leak is found, a decision is made, typically by the Unified Command, to either repair (in the case of a minor containment breach) or to abort the decontamination cycle (in the case of a major breach or a leak that cannot be safely repaired in the presence of the decontaminant). Action is based on action levels spelled out in the Ambient Air Monitoring Plan. The levels are set through negations with the EPA and are typically based on some fraction of the permissible exposure limit (PEL).

\section{F.4.5 Choice of Gaseous Decontamination Reagents}

The TWG may recommend, and the UC will decide on the choice of, specific decontamination methods as a function of the details of the situation. Table F-5 is a comparison of the two major gaseous technologies currently available for large-scale decontamination, namely chlorine dioxide and vaporous hydrogen peroxide. Vaporous hydrogen peroxide has the advantage of a good compatibility profile and, thus, may be compatible with sensitive equipment. It has straightforward generation and monitoring, and undergoes catalysis to water and oxygen, thereby generating less waste. However, its penetration is limited, and it can be easily absorbed or broken down by many materials, requiring extensive facility preparation and the removal of sorptive materials. In addition, generation capacity is currently limited to volumes of 100,000 to $250,000 \mathrm{ft}^{3}$. This technology is currently most appropriate for smaller, more confined spaces and areas with sensitive equipment. However, the availability of multiple generators could enhance its applicability to large spaces. 


\section{APPENDIX F}

Table F-5. Comparison of chlorine dioxide and vaporous hydrogen peroxide fumigants.

\begin{tabular}{|c|c|c|}
\hline Factor & Chlorine dioxide $\left(\mathrm{ClO}_{2}\right)$ & Vaporous hydrogen peroxide (VHP) \\
\hline $\begin{array}{l}\text { Chemical/physical } \\
\text { properties }\end{array}$ & $\begin{array}{l}\text { Yellow-green gas, single electron oxidizing } \\
\text { agent, unstable in UV light, explosive at } \\
\text { concentrations }>10 \%\end{array}$ & $\begin{array}{l}\text { Nonflammable, colorless, nearly odorless } \\
\text { vapor, heavier than air }\end{array}$ \\
\hline $\begin{array}{l}\text { Largest volume fumigated } \\
\text { at one time }\end{array}$ & $\geq 14 \times 10^{6} \mathrm{ft}^{3}$ (Brentwood) & $2 \times 10^{5} \mathrm{ft}^{3}$ [Department of State (DOS)] \\
\hline Largest building fumigated & Brentwood $\left(\geq 14 \times 10^{6} \mathrm{ft}^{3}\right)$ & $\operatorname{DOS}\left(1.4 \times 10^{6} \mathrm{ft}^{3}\right)$ \\
\hline $\begin{array}{l}\text { Building fumigation } \\
\text { approach }\end{array}$ & Fumigation of entire facility at one time & Sequential fumigation of zones within facility \\
\hline $\begin{array}{l}\text { Number of facilities } \\
\text { fumigated }\end{array}$ & $\begin{array}{l}\text { Four (Hart Building sections, Brentwood, } \\
\text { Trenton, and AMI) }\end{array}$ & Two (DOS and GSA Bldg 410) \\
\hline Generation of agent & $\begin{array}{l}\text { Onsite reaction of precursor chemicals in } \\
\text { solution }\end{array}$ & $\begin{array}{l}\text { Onsite vaporization of hydrogen peroxide } \\
\text { solution }\end{array}$ \\
\hline $\begin{array}{l}\text { Quantity of precursor } \\
\text { chemical used at site }\end{array}$ & $\begin{array}{l}\text { Trenton: } 31,920 \mathrm{lb} \text { DREWCHLOR }(25 \% \\
\text { aqueous solution sodium chlorite) }\end{array}$ & $\begin{array}{l}\text { DOS: } \leq 6860 \mathrm{lb} 35 \% \text { solution of hydrogen } \\
\text { peroxide }\end{array}$ \\
\hline $\begin{array}{l}\text { Real-time monitoring of } \\
\text { fumigant }\end{array}$ & No (hourly samples) & Yes \\
\hline Penetration capability & Good & Limited \\
\hline Setup time & 6 months (Trenton) & 1.5 months (DOS) \\
\hline $\begin{array}{l}\text { Duration of fumigation } \\
\text { process }\end{array}$ & $\sim 20$ hours (whole building at once) & $\begin{array}{l}\sim 8 \text { to } 12 \text { hours (each zone within building); } 2 \\
\text { months to fumigate all zones }\end{array}$ \\
\hline $\begin{array}{l}\text { Mode of removing } \\
\text { fumigant following } \\
\text { fumigation }\end{array}$ & $\begin{array}{l}\text { Reaction of } \mathrm{ClO}_{2} \text { with sodium hydroxide and } \\
\text { sodium bisulfite (Hart, Trenton, Brentwood), } \\
\text { carbon scrubbing (AMI) }\end{array}$ & Catalytic breakdown to water and oxygen \\
\hline Special conditions & $\begin{array}{l}\text { All windows/openings must be covered to } \\
\text { prevent breakdown from UV light }\end{array}$ & $\begin{array}{l}\text { Numerous absorbers of VHP in building } \\
\text { materials, which lower effective concentration } \\
\text { of VHP }\end{array}$ \\
\hline Materials compatibility & $\begin{array}{l}\text { Effects on uncoated aluminum, copper, } \\
\text { carbon steel; effects on some polycarbonates/ } \\
\text { polyurethanes. Replaced circuit breakers at } 2 \\
\text { postal facilities }\end{array}$ & $\begin{array}{l}\text { Effects on copper, aluminum, steel after } \\
\text { extended exposure }\end{array}$ \\
\hline $\begin{array}{l}\text { Source reduction and Waste } \\
\text { disposal }\end{array}$ & $\begin{array}{l}\text { Good penetration capability of gas allows } \\
\text { for much of building's contents to be left in } \\
\text { place, thereby reducing waste for disposal. }\end{array}$ & $\begin{array}{l}\text { Because of vapor's limited penetration } \\
\text { capability, most of building's contents may } \\
\text { need to be removed, treated, and either } \\
\text { disposed or reused. }\end{array}$ \\
\hline Regulatory exposure limits & $\mathrm{PEL}^{1}: 0.100$ ppm; IDLH ${ }^{2}: 5.0$ ppm & PEL: 1.0 ppm; IDLH: 75 ppm \\
\hline $\begin{array}{l}\text { Biological indicator results/ } \\
\text { consequences }\end{array}$ & $\begin{array}{l}\text { Brentwood: } \sim 1.5 \% \text { positive/none; } \\
\text { Trenton: } \sim 1 \% \text { positive/more environmental } \\
\text { sampling near location }\end{array}$ & $\begin{array}{l}\text { DOS: All must be negative/refumigation (one } \\
\text { refumigation of zone) }\end{array}$ \\
\hline $\begin{array}{l}\text { Clearance environmental } \\
\text { sampling }\end{array}$ & All samples negative (Brentwood, Trenton) & All samples negative (DOS) \\
\hline
\end{tabular}

${ }^{1} \mathrm{PEL}=$ permissible exposure limit promulgated by the Occupational Safety and Health Administration as safe for 8-hour, timeweighted-average workplace exposures.

${ }^{2}$ Exposure level considered immediately dangerous to life and health for exposure of 15 minutes. 


\section{APPENDIXF}

Chlorine dioxide gas can be readily generated onsite and introduced into large, tented structures without subdividing, as is required with VHP. The gas penetrates better than VHP but breaks down in UV light and must be applied in complete darkness. Whereas porous materials can be treated in place, such materials (e.g., carpeting) absorb and consume more gas than nonporous surfaces and might be discolored. A fine salt residue might create a corrosion hazard for certain metals in the presence of moisture (e.g., copper circuit breakers). Overall, however, chlorine dioxide gas is the fumigant of choice for large-volume structures.

\section{F.5 Decontamination of Personal, Valuable, or Sensitive Items}

Chemical sterilization and irradiation chambers can be used to decontaminate personal or valuable items removed from a facility. Chemical sterilants may be used for smaller pieces of sensitive equipment. In chemical sterilization, chemicals such as ethylene oxide, paraformaldehyde, or chlorine dioxide are used to kill spores on discrete items placed in a sterilization chamber. Adequate aeration of items after treatment is required to move residual amounts of sterilant and any toxic byproducts that may have formed.

If the internal parts of machinery and equipment are sampled and found to be contaminated, then a gaseous or vaporized product could be selected. A crisis exemption for paraformaldehyde, methyl bromide, ethylene oxide, and chlorine dioxide could be requested for this purpose. Vaporous hydrogen peroxide could also be used if the equipment were opened up completely.

\section{F.5.1 Paraformaldehyde}

Powder or flakes of paraformaldehyde are depolymerized by heat to form paraformaldehyde gas, which poisons Bacillus anthracis spores. It is noncorrosive but is genotoxic and a probable human carcinogen. Efficacy is influenced by concentration, temperature, and humidity, which must be maintained throughout the required contact time of 16 to 18 hours. It cannot penetrate grease, oil deposits, or dense absorptive materials. It is neutralized by ammonium bicarbonate and removed via venting or aeration. Crisis exemptions were issued for paraformaldehyde on January 30, 2001, and on February 14, 2002 for use as a confined-space decontaminant.

\section{F.5.2 Ethylene Oxide}

Ethylene oxide (EtO) gas is an antimicrobial agent that penetrates Bacillus anthracis spore walls and destroys nucleic acids by alkylation. It is an odorless, colorless gas at room temperature with minimal corrosivity. It is flammable and explosive above 3.6\%. Ethylene oxide sterilization is carried out in a fixed or portable sterilization system. It is potentially incompatible with rubber, plastics, and certain organicbased coatings. Items to be treated are placed in a chamber and pre-conditioned (exposed to the elevated temperature and humidity levels required for treatment). During treatment, temperature, humidity, contact time, air convection, and load configuration affect sterilization efficacy. Ethylene oxide is pumped out of the treatment chamber and items aerated so that less than 1 ppm remains in the air (the OSHA occupational 8-hr time weighted average). Crisis exemptions were issued on December 7 and 17, 2001; on January 3 and 9 , 2002; and on February 26, 2003, for the treatment of individual items or mail potentially exposed to Bacillus anthracis spores. 


\section{APPENDIX F}

\section{F.5.3 Chlorine Dioxide}

During cleanup of the Hart Office Building, chlorine dioxide gas was identified as the best available reagent for fumigating mail and packages. Contractors removed items from congressional offices that were critical to congressional operations or were personal effects of significance. The items were bagged, tagged, and moved for offsite decontamination. Approximately 4,000 packages and other mail items were collected from mail rooms in congressional office buildings and transported offsite for decontamination using chlorine dioxide gas. (In addition, approximately 3,250 bags of critical items were transported to a company in Richmond, Virginia, for decontamination treatment using ethylene oxide, and drums of mail were sent to a facility in Lima, Ohio, for irradiation treatment.)

\section{F.5.4 Irradiation}

Irradiation sterilization techniques include exposure to high-energy electrons from particle accelerators or high-energy electromagnetic radiation in the form of $\mathrm{x}$ rays or gamma rays. Particle accelerators can be used to generate $\mathrm{x}$ rays in the form of bremsstrahlung radiation by placing a high-atomic-number conversion target in the electron beam. Radioisotopes of cobalt and cesium are the principal sources of gamma rays. Given a sufficient absorbed dose, all organisms, including spores of B. anthracis, are rendered unable to reproduce as a result of DNA damage. However, irradiation can also destroy magnetic media, such as film or videotape, and tends to be expensive. Potentially contaminated mail has been treated at electron beam and x-ray irradiation facilities in Ohio and New Jersey, but see Section 3.5.2.4 for limitations. The EPA does not have regulatory authority over irradiation because it is governed under the Federal Drug Administration's medical instrument regulations.

\section{F.6 Emerging Technologies}

\section{F.6.1 Joint Science and Technology Program}

One of the most comprehensive decontamination studies to date is being conducted under the umbrella of the Joint Science and Technology Chemical/Biological Decontamination Master Plan. This Master Plan represents the combined efforts of many federal agencies. The main objective of the Master Plan is to develop efficient decontamination technologies that ultimately will become commercially available systems. Of the many decontamination technologies under investigation, those with potential applications for interior equipment, exterior equipment, and sensitive equipment are most applicable to airports. The expectation is to have several functional technologies in place within the next two years.

\section{F.6.2 Chemical and Biological Information Analysis Center Review}

An extensive review of decontamination technology applicable to equipment, personnel, and sensitive equipment was recently published by the Chemical and Biological Defense Information Analysis Center (CBIAC 1999) entitled, Wide Area Decon: CB Decontamination Technologies, Equipment and Projects. This review covers the topics of skin and personal equipment decontamination, exterior equipment decontamination, sensitive and interior equipment decontamination, large-area decontamination, specific decontaminants, specific decontamination processes, and proactive decontamination. For this review, more than 20,000 records were examined, and the information was placed in a decontamination database. A 


\section{APPENDIXF}

domestic and international market survey identifies which technologies were available that could support current decontamination technologies.

\section{F.7 Crisis Exemption}

Antimicrobial pesticides are used to control harmful microorganisms - including bacteria, viruses, or fungi-in many situations. The most important in the present context is the use of antimicrobial pesticides on inanimate objects and surfaces primarily in indoor environments. Antimicrobial products have traditionally included:

- Sanitizers - substances that significantly reduce the bacterial population in the inanimate environment, but do not destroy or eliminate all bacteria or other microorganisms.

- Disinfectants - substances that destroy or eliminate a specific species of infectious or other public health microorganism, but not necessarily bacterial spores, in the inanimate environment.

- Sterilants - substances that destroy or eliminate all forms of microbial life in the inanimate environment, including all forms of vegetative bacteria, bacterial spores, fungi, fungal spores, and viruses.

The EPA regulates antimicrobial pesticides under the Federal Insecticide, Fungicide, and Rodenticide Act (FIFRA). Under FIFRA, all antimicrobial pesticides must be registered and labeled for specific uses. Under Section 18 of FIFRA, the EPA "may exempt any Federal or State agency from any provision of this Act if the Administrator determines that emergency conditions exist which require such exemption." Normally, a Federal or State agency would submit an application for a FIFRA exemption to the EPA for review and approval. If the EPA approves the request, it would issue either a specific or a public health exemption, as appropriate. However, if the emergency is of such urgency that a Federal or State agency does not have enough time to submit an application for exemption and wait for EPA approval, then the Federal or State agency may issue a crisis exemption, which is effective for 15 days. For the crisis exemption to be extended beyond 15 days, the Federal or State agency must submit an application for exemption to the EPA.

There are currently no pesticides registered and labeled for use on facilities contaminated with B. anthracis. To obtain a crisis exemption from the EPA for the unregistered use of a pesticide against $B$. anthracis, a Federal or State or agency must submit a written request describing the antimicrobial product(s) to be used; how, when, and where they will be used; data demonstrating efficacy of the product for the intended purpose; and how human health and safety will be protected. In the crisis exemptions issued for anthrax cleanups, this information has been contained in a Remediation Action Plan (RAP), a clearance sampling and analysis plan (SAP), and an ambient air monitoring plan (AAMP), submitted to the EPA with a cover letter. Figure F-1 is a template for a crisis exemption letter. Before issuing the exemption, the EPA will perform a multidisciplinary risk assessment of the requested use, relying on data supplied for the pesticide.

If, during a review, the EPA notes any adverse human health or environmental concerns, the EPA may deny the exemption request. However, if the EPA believes that the proposed use of an antimicrobial product will be effective and will protect human health and the environment, then it will issue a crisis exemption. When the EPA has issued a crisis exemption, if the EPA determines that use of the product is needed beyond the 15-day use period, the EPA will complete an application for a public health exemption on behalf of the requesting entity, which allows the crisis exemption to continue in effect until it is either withdrawn or the EPA issues a public health exemption. 


\section{Figure F-1. Template for Crisis Exemption letter.}

\section{Month DD, YYYY}

Airport Letterhead

Team Leader, Emergency Response Team, Registration Division

Office of Pesticide Programs

U.S. Environmental Protection Agency

1801 South Bell Street Crystal Mall 2, Room 308

Arlington, VA 22202

RE: Crisis Exemption Request for the use of $<$ product $>$ in/on $<$ action $>$

Pursuant to 40 CFR 166.40, the San Francisco International Airport is requesting a crisis exemption from the U.S.

Environmental Protection Agency for the use of <product> (CAS number if available) (USEPA registration number if available) as a pesticide in the decontamination of $<$ items/facility $>$ contaminated with $<$ high/low $>$ burdens of Bacillus anthracis at the San Francisco International Airport. The contaminated <items/facility> are/is located at: (Give location of stored items to be decontaminated or facility to be decontaminated).

Our objective is to use <product $>$ to decontaminate $<$ items/facility $>$ that are potentially contaminated with Bacillus anthracis spores. [If items are to be transported to an offsite facility for treatment, provide the location where the items will be treated] The decontamination and verification process and procedures are described in the attached Remediation Action Plan, Sampling and Analysis Plan, and Ambient Air Monitoring Plan (unless the Ambient Air Monitoring Plan is a section within the Remediation Action Plan), which were prepared by <list preparer and any important team members such as EPA, FDA, technical consultants>.

Information required for a crisis exemption application under FIFRA Section 18 for the use of $<$ product $>$ is summarized below.

Alternative Methods of Control

Give rationale why selected product is the best for proposed use, and briefly describe other methods considered.

Type of Exemption Requested: Crisis Exemption

Description of Product

Common Chemical Name (active ingredient)

CAS No.

Product Name and Manufacturer

EPA registration number, if applicable

Formulation (Give chemical formula)

For questions concerning administration of the crisis exemption, contact $<$ name $>$ (phone number) or $<$ name $>$ (phone number). Please contact us immediately if you need further documentation or have other questions.

Sincerely,

Signature Block

Attachments:

Proposed Remediation Action Plan

Proposed Sampling and Analysis Plan

Proposed Ambient Air Monitoring Plan 


\section{APPENDIXF}

If pesticide products become registerd for inactivation of $B$. anthracis spores in the future, the three plans identified above will likely be required by the product's labeling, but the plans would no longer be submitted to the EPA for approval; rather, the Incident Commander would approve them. If fumigation is used, an Emergency Response Plan is also recommended although such a plan in not required for a cirsis exemption.

\section{F.8 Indemnification}

Most cleanup contractors will require some sort of indemnification prior to responding to a BWA cleanup. For example, numerous uncertainties about the use of chlorine dioxide gas for decontamination existed following the anthrax incidents of 2001. The contractor who was tasked to fumigate the Hart Office Building using chlorine dioxide gas would not start removal procedures without receiving indemnification from the EPA against liability for damages. Another contractor, whose responsibilities included placing the materials to test for the presence of $B$. anthracis during fumigation, received indemnification terms similar to those granted to the first contractor, but with significantly smaller compensation amounts. Insurance could be an alternative to indemnification.

\section{F.9 References}

CBIAC (1999), Chemical and Biological Defense Information Analysis Center, Wide Area Decon: CB Decontamination Technologies, Equipment, and Projects, CR-99-10.

EPA (September, 2002), U.S. Environmental Protection Agency, Challenges Faced During the Environmental Protection Agency's Response to Anthrax and Recommendations for Enhancing Response Capabilities, A Lessons Learned Report, U.S. Environmental Protection Agency, Washington, D.C.

GAO (June, 2003), U.S. General Accounting Office, Capitol Hill Anthrax Incident, EPA’s Cleanup Was Successful. Opportunities Exist to Enhance Oversight, GAO Publication GAO-03-686.

http://www.epa.gov/opp00001/factsheets/chemicals/vhp_factsheet.htm http://www.epa.gov/pesticides/factsheets/chemicals/chlorinedioxidefactsheet.htm http://www.eurekalert.org/pub_releases/2003-03/asfm-vhp030703.php http://www.loudoun.gov/general/anthraxfaqs.htm\#gen NRT (July, 2005), National Response Team, Technical Assistance for Anthrax Response, Interim-Final Draft, Phase I Update; available only by electronic means.

Silva, J. (2003), Vaporized Hydrogen Peroxide May be the Best Method of Anthrax Decontamination (abstract), American Society for Microbiology Biodefense Meeting, March 10, 2003; available at www.asmbiodefense.org. 


\section{Collection, Treatment, and Disposal of Bacillus anthracis-Contaminated Wastes}

Decontamination activities associated with remediation of biological contamination will generate various types of solid and liquid wastes. How the wastes are managed will depend on the characteristics of the waste, including types, amounts, and locations, and the waste disposal strategies that will be determined by key stakeholders and responsible regulatory authorities.

Waste management considerations, including treatment and ultimate disposal, should be factored into decisions related to the development of an overall decontamination strategy, and a disposal plan should be developed as part of the Remediation Action Plan (see Appendix J, Section 6). Bacillus anthracis-contaminated wastes are not regulated under Subtitle $\mathrm{C}$ of the Resource Conservation and Recovery Act (RCRA), but they should be handled with caution because of the potential for exposure to an infectious agent. In some states and localities, the wastes are considered medical waste or infectious substances with special requirements for handling and disposal. Therefore, it is essential to contact the state or local regulatory agency early to determine what state or local requirements apply and what treatment and disposal options are available. State authorities have the primary responsibility to regulate and oversee management of wastes that may be contaminated with an infectious agent, such as Bacillus anthracis. It is also advisable to establish contact early in the process with other important waste-disposal stakeholders, such as publicly owned wastewater treatment operators and landfill, incinerator, and sterilization facilities. Pre-negotiate disposal contracts, if possible.

Bacillus anthracis-contaminated waste may be subject to Department of Transportation (DOT) and other agency requirements applicable to the transportation of infectious substances. DOT and other relevant agencies should be consulted regarding any questions about transporting wastes that are contaminated with infectious agents. In this appendix, the term "treatment" means subjecting a waste to a process that will reduce or destroy Bacillus anthracis spores prior to disposal offsite. In most cases, Bacillus anthracis-contaminated wastes should be treated onsite to reduce or destroy spores, tested to confirm treatment effectiveness, and treated further, if necessary, until post-treatment sampling shows no indication of remaining viable spores. If such a process is followed, treated wastes may possibly be disposed of as municipal solid waste or wastewater, given approval from appropriate state and local authorities.

When total elimination of spores cannot be confirmed, wastes must be properly packaged and transported to a state or locally approved waste-treatment facility capable of destroying any remaining spores. Depending on the capacity of available offsite facilities and the size and volume of wastes to be treated, either medical or other equivalent types of waste-treatment facilities may be used. State or local approval, including approval of any necessary facility-specific handling protocols, should be obtained for all offsite treatment.

Appendix G discusses the management of wastes from Bacillus anthracis-contaminated sites, including:

- The need to notify waste and recycling service providers of potential contamination.

- The possible types of contaminated wastes, including wastewater.

- How to manage wastes that are, or may be, contaminated.

Appendix G deals with Bacillus anthracis contamination, which is considered to be a "worst-case" scenario. Waste disposal considerations will vary depending on the specific biological agent involved in an attack and its persistence in the environment. 


\section{APPENDIX G}

\section{G.1 Notification of Waste and Recycling Service Providers}

If there is reason to believe that a building or site may be contaminated, authorized facility personnel should immediately notify their waste haulers and recyclables collectors. Facility personnel should provide the suspected date on which contamination may have occurred, and update the waste and recyclables collector as further information becomes available. If contamination is confirmed, pickups of solid waste or recyclables from potentially contaminated areas may need to be discontinued. If contaminated waste has already been picked up and disposed of, sampling and decontamination of the transporters and waste disposal facility may be necessary.

\section{G.2 Types of Waste}

The most common types of wastes resulting from remediation of Bacillus anthracis contamination include:

- PPE and other materials used in assessment and decontamination;

- Debris intended for disposal, which could include:

- Small materials removed from the building, such as books, magazines, papers, pictures and wall hangings.

- Small equipment and office items, such as staplers, telephones, and hand tools.

- Office furniture (e.g., file cabinets) that may contain large amounts of paper.

- Vendor merchandise, such as clothes, novelty items, toys, cosmetics, and electronic devices.

- Luggage.

- Large, durable materials removed from the building, such as furniture, computers, luggage carts, chairs, display boards, and baggage-handling equipment.

- Building and decorating materials, such as carpeting, ceiling tile, insulation, duct work, wallboard, and stanchions. (The quantities and level of contamination of such wastes are closely tied to decontamination or fumigation strategies.)

- $\quad$ Filters removed from the building's HVAC system.

- Trash, food, and other unwanted materials at the site at the time of contamination.

- Wastewaters generated during decontamination, including chemicals used to treat PPE, subsequent rinses, and air-scrubber waters associated with fumigation.

\section{G.3 Waste Management}

This section outlines practices for onsite treatment or packaging, storage, transport, offsite treatment, and final disposal of wastes from Bacillus anthracis-contaminated sites. If sampling for the extent of contamination confirms the absence of Bacillus anthracis in specific, confined locations, waste generated exclusively from the Bacillus anthracis-free areas may be managed as municipal solid waste. To avoid unnecessary extra handling, waste from such areas must be physically segregated from waste removed from potentially or known contaminated areas of a facility.

All articles and structural materials within contaminated area(s) (hot zones) should be categorized as to whether they may be:

- Treated in place and reused (without replacement). 
- Treated in place and removed (and replaced).

- Removed for separate treatment and/or further sorting for reuse or disposal.

Decisions about how to categorize contaminated articles and materials should be determined by analyzing several factors, such as:

- What chemical decontamination agent will be used and whether treatment in place is feasible.

- Whether an article or material might be damaged by the treatment.

- How the cost of treating or replacing an article compares to the cost of disposing and replacing it.

In the past, some cleanups involved removing all articles and materials from a structure (e.g., Department of State Sterling mail facility), whereas others involved only partial or minimal removal (e.g., Brentwood, Hamilton, and AMI Building). In general, the more articles and materials that are treated in place, the more economical the overall remediation process will be. The decontamination technology selected for facility cleanup may have a major impact on quantities and contaminant levels of materials removed for disposal. The practices described below are options for minimizing the risks of handling and disposing of wastes.

\section{G.3.1 Onsite Treatment}

In general, wastes will be treated before being moved to a storage location or offsite for further treatment or disposal. Options for treating wastes onsite include the use of HEPA vacuuming, antimicrobial solutions, and fumigation. It may be necessary to use more than one option to effectively deal with certain types of contamination.

To confirm the effectiveness of treatment, wastes should be sampled after treatment. Samples should be cultured and analyzed for spore growth. If there is no indication of spore growth in any of the samples, then treatment is considered to be effective. However, some porous materials may not be amenable to sampling to attain a sufficient degree of confidence to ensure that no spores remain viable.

If treatment effectively eliminates all viable spores, nonhazardous waste (such as couches, bulletin boards, tables, and carpets) can then be disposed of as municipal solid waste as long as appropriate state or local officials allow it. However, objects that are otherwise nonhazardous can inadvertently be made "toxic" through the copious application of certain antimicrobial agents. The quantity or concentration of a given antimicrobial agent that makes a waste hazardous should be ascertained before use.

Decontaminated waste should be segregated physically or strategically into the following categories:

- Hazardous waste (such as stored dry chemicals, computer monitors, and fluorescent light tubes).

- Radioactive waste (such as smoke detectors and tritiated exit signs).

- Biohazardous waste (for objects deemed too porous or having too much surface area to be effectively treated onsite).

- Waste requiring subsequent treatment prior to disposal (decontaminated materials for which definitive sterilization declarations cannot be rendered).

The airport team managing the remediation effort must characterize all wastes and manage each one according to applicable Federal, state, and local regulations. 


\section{APPENDIX G}

\section{G.3.1.1 Hazardous and Radioactive Waste}

Items that contain hazardous or radioactive components at environmentally significant levels require special handling, even after viable microbial agents have been removed. Small waste items that are hazardous or radioactive, by DOT definition, must be packaged and transported pursuant to DOT regulations, as prescribed in 49 CFR Parts 100-185. Sterilized, small and large hazardous waste items that are normally recycled, and that contain interstitial spaces, should not be recycled, but managed as hazardous waste. Large DOT-regulated items may require placement in DOT-approved bulk containers.

\section{G.3.1.2 Biohazardous Waste}

Biohazardous waste designated for offsite treatment should be pre-treated by vaporous hydrogen peroxide, chlorine dioxide gas, or another appropriate method. (See Section F.7 in Appendix F regarding FIFRA crisis exemptions.) Such biohazardous waste should be wrapped in the exclusion zone, double-wrapped in the warm zone, and relayed to clean-zone workers for loading onto the designated transport vehicle. If necessary (and if space allows), pre-treated biohazardous articles that are wrapped in the exclusion zone can be accumulated in that area pending the arrival of a transport vehicle. Waste articles that cannot be deemed nonbiohazardous must be managed as biohazardous. The DOT does not authorize the use of bulk packaging for large quantities of biohazardous waste. When biohazardous waste must be shipped in bulk quantities (volume capacities greater than 119 gallons or net mass exceeding 882 pounds), the shipper must apply to the DOT for a packaging exemption, as provided in 49 CFR 107.105.

\section{G.3.2 Storage}

Bacillus anthracis-contaminated waste may be stored for further treatment, or pending test results, in sealed containers that are appropriately labeled. If waste is temporarily stored before transport to offsite disposal, it must be in containers that meet the DOT Division 6.2 (Infectious Substances) packaging requirements described below. The storage area must provide weather protection and prevent access by unauthorized individuals or by vermin.

\section{G.3.3 Transportation}

Bacillus anthracis is one of the biological agents covered under the Select Agent Rule (42 CFR 72.6). The CDC administers the Select Agent Program. Before transporting Bacillus anthracis-contaminated waste to an offsite treatment facility, generators should contact the Select Agent Program within CDC's Office of Health and Safety (Ph: 404-639-4418) for any special handling and transportation requirements that may apply. General information on the Select Agent Rule can be found at: www.cdc.gov/od/ohs/Irsat.htm.

Bacillus anthracis-contaminated waste may not be transported as a regulated medical waste. Commercial transportation of Bacillus anthracis-contaminated waste must meet requirements in the DOT's Hazardous Materials Regulations (HMR 49 CFR Parts 171-180). These requirements do not apply to waste that has been treated so that the Bacillus anthracis spores are destroyed. If complete destruction of spores cannot be demonstrated, then the waste should be handled as follows:

- Packaging ( $\S 173.196,178.609)$. Use triple packaging that meets the performance requirements in $\$ 178.609$ (e.g., drop test, water immersion test) and consists of the following components:

- Water-tight primary receptacle. 
- Water-tight secondary receptacle.

- Sufficient absorbent material between the primary and secondary receptacles to absorb the entire contents if the material is liquid.

- Outer packaging of adequate strength for capacity, mass, and intended use.

- Itemized list of package contents placed between the secondary receptacle and outer packaging.

- Labeling (\$172.400). Label the package with an "INFECTIOUS SUBSTANCE” label.

- Marking ( $\$ 172.301)$. Mark the package with the words "Infectious substance, affecting humans, UN 2814, (Bacillus anthracis), 6.2" and with the name and address of the consignor or consignee. The labeling requirements permit a generic name (e.g., Bacillus species) to be used in place of the technical name (e.g., Bacillus anthracis).

- Shipping documents $(\S \S 172.202,172.203,172.204,172.604)$. Prepare a shipping paper that includes the following information: "Infectious substance, affecting humans (Bacillus anthracis), 6.2, UN 2814," and the quantity being shipped. Include the following certification: "This is to certify that the above-named materials are properly classified, described, packaged, marked, and labeled, and are in proper condition for transportation according to the applicable regulations of the Department of Transportation." Also, include an emergency telephone number that is staffed by a person familiar with the material being shipped and with emergency measures to be taken in the event of a leak or other emergency.

If Bacillus anthracis-contaminated waste cannot be placed in packaging that meets the HMR requirements because of its size or form, then an exemption from the HMR is needed to transport the material. The exemption application should specify the type of packaging proposed for shipping the object. The packaging must be adequate to ensure that spores cannot escape during transportation. Waste haulers must be appropriately licensed or certified by the state, where such programs exist. For details on DOT requirements go to: www.hazmat.dot.gov/guide_anthrax.htm or call the Hazardous Materials Information Center at 202-366-4488 (option 1). Appropriate state transportation regulations should also be examined.

\section{G.3.4 Offsite Treatment}

Depending on uncertainties related to the effectiveness of onsite treatment, the capacity of available offsite facilities, and the size and volume of wastes to be treated, several alternative offsite methods are available to complete the destruction of any remaining spores. Suitable alternatives include:

- Medical waste incinerators.

- Medical waste autoclaves.

- State-approved, alternate, medical waste-treatment technologies.

- Municipal solid-waste incinerators.

- Hazardous-waste combustion devices.

Arrangements should be worked out with state and local regulatory agencies and the owner/operator of a facility to establish a site-specific protocol to ensure that the material is safely received, unloaded, and placed into the device. Wastes should not be shredded or broken; they must be packaged in a manner that minimizes handling, and they must be separated from other wastes. Treatment units should be assessed for appropriate operational parameters (e.g., proper temperatures, retention times, air flows, pressure, and feed 


\section{APPENDIX G}

and seal systems that prevent spore emissions). A facility's compliance history should be examined as an indicator of its ability to maintain operational conditions.

It may be necessary to develop an alternative strategy for storing, packaging, transporting, and disposing of wastes that cannot be treated onsite and that physically cannot be handled in available medical waste facilities. Such a strategy should be developed jointly by the IC or UC, the USEPA, the DOT, and affected states and localities.

\section{G.3.5 Disposal}

If appropriate state and local officials approve, ash or other residues from Bacillus anthracis treatment via combustion, autoclaves, or other state-approved treatment technologies may be disposed of as municipal solid waste.

\section{G.4 Liquid Waste}

Large quantities of liquid waste or wastewater will be generated in the remediation of biological agents. All equipment, materials, and personnel leaving a site must be decontaminated by washing with an aqueous disinfectant solution. Options considered for disposal of wastewaters during past Bacillus anthracis cleanups included incineration, hazardous waste landfills, and treatment at the local wastewater treatment plant or publicly owned treatment works (POTW). The potential generation of large volumes of wastewater makes the POTW an important stakeholder that needs to be involved early in a remediation process when decontamination decisions are made. The Planning Section should work with the POTW to ensure that the protocol for disinfection of wastewater meets the POTW's needs, and that wastewater is compatible with its process. If incompatible agents are present in wastewater, it would be captured, if possible, and treated to remove the incompatible agents prior to discharge, or shipped to an offsite treatment and disposal facility. Guidelines for discharging Bacillus anthracis-decontamination wastewater to POTWs can be found in Appendix E of the National Response Team's Anthrax Technical Assistance Document at www.nrt.org. The guidelines include recommendations related to:

- Notification of proper authorities.

- Health and safety considerations.

- Disinfection.

- Chlorine residual and other parameters of concern.

- Sampling.

- Laboratory coordination.

- Packaging and transporting samples.

- Discharge authorization letters and discharge permits.

The guidelines also provide information on the effect of $\mathrm{pH}$, chlorine concentration, temperature, and contact time for inactivating spore suspensions of Bacillus anthracis in wastewater.

Many wastewater treatment facilities apply their sludges for various land applications. Depending on the agent in question and the decontamination method used, technical or political hurdles may need to be overcome before using the wastewater treatment facility to dispose of decontamination wastewater. 


\section{APPENDIXG}

Decontamination wastewater generated in areas where storm water is not captured and routed to the industrial wastewater treatment system needs to be managed to eliminate, or at least minimize, the potential for discharge into surrounding wetlands, bay, or other bodies of water. Prior to a BWA event, the drains directly to surrounding bodies of water should be identified, and a means to block the drains should be maintained onsite. If agent decontamination needs to occur in such areas, the storm drains should be blocked. The decontamination wastewater should be captured and diverted to the industrial or sanitary sewer system. Wastewater composition will determine in which sewer system the re-directed effluent will be managed.

\section{G.5 Summary}

Many variables influence the management of solid and liquid waste streams generated at the scene of a bioterrorist event. Carefully planned waste-management strategies need to address waste minimization, site and waste characterization issues, decontamination and segregation concerns, and the wastewater management infrastructure. It is especially important that:

- State and local regulatory agencies be contacted early in the process for assistance in establishing and approving a waste-disposal strategy.

- Best management practices be used onsite.

- Potentially contaminated wastes be appropriately packaged, labeled, and transported.

- State-approved, alternative, offsite treatment devices, such as municipal solid waste incinerators and hazardous waste combustion devices, be operated at conditions equivalent to or exceeding those for medical wastes. Check with your state agency to determine what those conditions are.

Attention to these issues will incur minimal environmental degradation and accelerate, rather than delay, the return of an airport to operational status. 



\section{APPENDIX H}

\section{Sampling Zone and Sampling Unit Information Forms for Characterization and Clearance}

Sampling zones and units are described in detail in Chapter 2, Section 2.2.6, and in Appendix D, Section D.2.2. The concepts of sampling zones and sampling units are briefly reviewed here.

A sampling zone is a discrete portion of an airport within which sampling is conducted. Sampling zones should be identified as part of an airport's pre-planning and are based on the physical structure of an airport. Sampling zones should be chosen in a way that will help remediation planners and workers keep track of what sampling has been done, where it has been done, and what information has been acquired from sampling.

Characterization zones are made up of one or more sampling zones, and their selection depends on the details of a specific event. If fumigation is anticipated, a good basis for defining fumigation zones is to find rooms, areas, or sections that can be isolated (sealed off) from each other, each of which either will or will not be decontaminated in its entirety. The clearance decision can be made independently for each such zone because each one is isolated from every other. The basis is primarily physical, that is, areas are physically separated from each other by sealed barriers.

Sampling units are defined within sampling zones. A sampling unit is any structure, or set of one or more objects, that can be sampled and evaluated collectively as a unit. For example, in a concourse, the floor would be considered to be one sampling unit; walls (if sampled at all), air-returns, and ticket-counters would each be separate sampling units. There will be at least one, and probably more than one, sampling unit within each sampling zone.

This appendix contains templates that are designed to help workers keep track of sampling zones and units, remind investigators of the kinds of information that should be collected, and help samplers record information about sampling zones and units. 


\section{APPENDIX H}

\section{Sampling Zone Form-Characterization}

Complete one form for each sampling zone

\section{Sampling zone code}

Sampling zone name

Choose a sampling zone code (a short abbreviation that can be used as part of sample names), and a short descriptive, user-friendly name.

\section{Sampling zone description}

Describe the zone (size, shape, types of activities it is used for, and other relevant information).

\section{Incident-related information about this zone}

For this zone, summarize information from the First Response Phase and other assessments described in Section 2.2 of the Remediation Guidance document. Information applicable only to specific sampling units within the zone should be on the sampling unit information form.

\section{Assessment of likelihood of contamination in this zone and why}

Is this zone contaminated? Choose one of the following categories, or write a description. Decide how much confidence can be placed in the assessment.

1. Contamination is confirmed or assumed. Decontamination will definitely be carried out.

2. Contamination is highly likely. A decision is required on whether to assume this zone is contaminated and proceed with decontamination, or whether to characterize to find out.

3. Contamination is uncertain. Characterization is needed.

4. It is plausible that the zone is probably not contaminated. A decision is required on whether to assume it is not contaminated, or whether to characterize to find out. If the former, a decision is needed on whether a full clearance process, including clearance sampling, is necessary. 


\section{Based on the above assessment, identify the type of characterization to be performed}

Identify hypotheses to test, questions to answer, or decisions that need to be supported with sampling data. Write your own, or use the following:

Decide whether

- Sufficient information exists to design the decontamination without additional sampling, or

- Information from characterization sampling is needed to design the decontamination.

If the latter, choose one or more of the following characterization goals:

- Determine whether or not contamination is present (i.e., if presence is uncertain).

- Confirm the absence of contamination (i.e., if presence is believed unlikely).

- If fumigation is expected, determine what fumigant level needs to be maintained (concentration $\times$ time) if presence is confirmed or assumed.

- Identify any areas that should receive surface decontamination (washing) either as the only decontamination method or as extra decontamination prior to fumigation (if it is believed that such areas can be identified, and if their contaminant levels are expected to be so high that surface decontamination is worthwhile).

Other potential purposes of characterization

- Search for hotspots.

- Develop a contaminant map.

- Sample locations having high levels for future comparison with clearance samples (for targeted sampling during clearance).

See Appendix D, Section D.3, and Section 2.3 of the Remediation Guidance document.

\section{List sampling units within this zone}

Prepare a sampling unit form for each potential sampling unit.

Assess each sampling unit. Decide whether or not sampling the unit will contribute to testing the hypotheses, answering questions, or supporting the decisions.

For each sampling unit selected for sampling, choose a sampling strategy: judgmental, random, or statistical (may use more than one). 


\section{APPENDIX H}

\section{Sampling Unit Form-Characterization}

Complete one form for each sampling unit within each sampling zone

\section{Sampling zone designation}

State the name of the sampling zone containing this sampling unit.

\section{Sampling unit code}

Sampling unit name

Choose a sampling unit code (an abbreviation that can be used as part of sample names), and a short, descriptive, userfriendly name.

\section{Sampling unit description}

Include any useful descriptive information (e.g., hard, porous, smooth, rough).

\section{Incident-specific information}

Provide any information specific to this sampling unit that pertains to this particular incident and that is not already described in the sampling zone form.

\section{Choose a sampling strategy}

Decide whether sampling this unit will help test the hypotheses, answer the questions, or support the decisions identified for the zone. If sampling is to be done, choose:

- A sampling strategy based on zone- and unit-specific information. Options include one or more of judgmental, random, and statistical sampling. See Appendixes D and E.

- The sampling protocol to be used (e.g., swab, wipe, or vacuum). The method must be appropriate to the physical material (type of surface - smooth or not, porous or not) and type of material (wood, carpet, metal, or HVAC filter). Include details, such as the exact surface area to be wiped, if wipes are used.

- The sample handling protocol to be used. Include details, such as how to package the sample, decontaminate the outside of the sample container, label, document, and so forth.

- The analytical method to be used.

- The types and number of quality control samples to be collected. 


\section{APPENDIX H}

\section{Sampling Zone Form-Clearance}

Complete one form for each sampling zone

\section{Sampling zone code}

Sampling zone name

Same as characterization, unless sampling zones are changed for clearance.

\section{Sampling zone description}

Same as characterization, unless sampling zones are changed for clearance.

\section{Characterization-phase information about this zone}

Summarize any characterization phase information that is relevant to clearance planning. Refer to the characterization sampling zone forms.

\section{Decontamination-phase information about this zone}

Summarize information from the decontamination phase that is relevant to clearance planning. Include any processmonitoring measures, such as fumigant concentration, temperature, humidity, and bio-indicator strip results, as applicable. Explicitly state whether the decontamination process met its design criteria in this zone (otherwise, the next activity should be more decontamination, not clearance).

\section{List sampling units within this zone}

Same list as characterization phase, unless zone or sampling unit definitions have been modified.

1. For each sampling unit in the zone, decide whether or not to sample.

2. For each unit being sampled, choose a sampling strategy (targeted, biased, random, or statistical). See Appendixes D and E. 


\section{Sampling Unit Form-Clearance}

Complete one form for each sampling unit

\section{Sampling zone designation}

State the name of the sampling zone containing this sampling unit.

\section{Sampling unit code}

Sampling unit name

Same as characterization, unless sampling units are changed for clearance.

\section{Sampling unit description}

Same as characterization, unless sampling units are changed for clearance.

\section{Incident-related information}

Describe any information acquired since characterization that is specific to this sampling unit.

\section{Choose a clearance sampling strategy}

Decide whether sampling this unit is necessary to support the clearance decision. If so, then

- Decide where to sample, what to sample, how many samples to collect. Options (see Appendix D) include, but are not restricted to, one or more of the following:

- Targeted sampling

- Biased sampling

- Random sampling

- Statistical sampling (see Appendix E)
i. Detection sampling
ii. Confirmation sampling
iii. Hot spot search.

- Specify the sampling method to be used (e.g., swab, wipe, or vacuum). The method must be appropriate to the physical material (type of surface - smooth or not, porous or not) and type of material (wood, carpet, metal, or HVAC filter). Include details such as the exact surface area to be wiped, if wipes are used.

- If aggressive air sampling is used in this sampling zone, include a sampling unit form to represent the aggressive air sampling. Aggressive air sampling encompasses the entire sampling zone.

- The sample handling protocol to be used. Include details, such as how to package the sample, decontaminate the outside of the sample container, label, document, and so forth.

- The analytical method to be used.

- The types and number of quality control samples to be collected. 


\section{APPENDIX}

\section{Annotated Characterization Sampling Plan Template}

This appendix describes the basic components required for a complete Characterization sampling and analysis plan (Characterization SAP). Part 1 of the template establishes the background and context for the Characterization SAP. Part 2 suggests an approach for deciding about sampling activities and documenting those activities. Part 3 provides supporting reference information. The objective of the template is to help ensure that no important information or considerations are omitted during planning, and to save time by providing a starting point for developing a written Characterization SAP. 


\section{APPENDIX}

\section{Part 1. Background Information}

\subsection{Brief Introduction}

Write a brief overview of the situation. Copy, update, and insert any overview information relevant to this incident if already written for other purposes.

\subsection{Statement of Purpose}

Write a general statement of purpose. Provide information needed to decide where to decontaminate, what to decontaminate, and how to decontaminate.

\subsection{Statement of Authority}

Describe applicable state and Federal laws under which the cleanup activity takes place. Include applicable agreements with, or directives issued by, relevant state or Federal agencies.

\subsection{List and Description of Involved Parties}

Refer to the concept of operations in Figure 1-4, and identify organizations and individuals responsible for developing and performing the Characterization SAP. Include:

- Incident Commander or Unified Commander (IC or UC).

- Sampling group (Operations section).

- Environmental unit (Planning section).

- Technical Working Group (Environmental unit).

- Technical consultants (e.g., analytical chemist, dispersion modeler, statistician, industrial hygienist, toxicologist, structural engineer, HVAC engineer, and fumigation engineer).

\subsection{History of Event and Actions To Date}

Describe the event to date: what happened, what is known, what is suspected, and results from First Response Phase. Record detailed, zone-specific information in Part 2 on the sampling zone information forms. 


\section{APPENDIX}

\section{Part 2. Characterization}

If sampling zones were identified as part of pre-planning, obtain the list of zones. If not, work with airport personnel to identify sampling zones now (See Appendix D and Chapter 2, Section 2.2.6). Use sampling zone templates (Appendix $\mathrm{H}$ ) as a tool to record characterization information and decisions.

\subsection{Develop a Sampling Plan for Each Sampling Zone}

For each sampling zone:

- Assess available information as described in Chapter 2, Section 2.2.

- Decide what hypotheses need testing, questions need answering, or decisions need to be supported (see Chapter 2, Section 2.3.1; Appendix D, Section D.2.4, and sampling zone forms in Appendix H).

- Decide what kind of sampling will test the hypotheses, answer the questions, or provide information to support the decisions (see Chapter 2, Section 2.3.2; Appendix D, Section D.2.4).

- Record assessments and decisions on the sampling zone forms (Appendix H).

\subsection{Interpretation}

To the extent possible, decide in advance what kinds of results will determine subsequent actions. That is, describe how the information will be used. 


\section{APPENDIX I}

\section{Part 3. Supporting information}

\subsection{Sampling Protocols}

Provide detailed instructions for how to collect samples. Protocols may be written in full or incorporated by reference. Include all methods that will be used. See Appendix C.

\subsection{Quality Control Samples To Be Collected}

Specify types and frequencies of quality control samples required (e.g., collocated samples, trip blanks, pure blanks, and spikes). Quality control may be the responsibility of contractors.

\subsection{Sample-Handling Protocols}

Specify how to collect, package, and document samples, and how to deliver samples to the laboratories. Include how sample handling is incorporated in environmental safety and health (ES\&H). See Appendix C.

\subsection{Analytical Methods}

Specify how the laboratories are to analyze the samples. Include quality control procedures required of the laboratories. See Appendix C.

\subsection{Data Management}

Describe sample-naming conventions, which may have been developed as part of the airport's pre-planning. If not, develop them now. Specify how results will be received from laboratories and linked to sample locations and sample collection information, how results will be stored (e.g., computer database), and facilities for data review. Data management may be the responsibility of contractors.

\subsection{Data Validation}

Summarize how results will be assessed for data quality. Data validation may be the responsibility of contractors.

\subsection{Sampling Design}

Describe rationales of the selected sampling strategies, i.e., the reasoning leading to choices of where to sample and how many samples to collect. Focus on concept. Full technical details, especially of probability-based designs, can be incorporated by reference.

\section{8. $\quad$ Enviornmental Safety and Health (ES\&H)}

Describe the levels of personal protective equipment (PPE) required for various sampling activities. Describe other actions necessary to ensure safety and health. Refer to the written Health and Safety Plan (HASP) where appropriate. 


\section{APPENDIX J}

\section{Remediation Action Plan}

Attached is a template for a Remediation Action Plan (RAP) to be filled out in the event of a biological warfare agent (BWA) release at a major transportation facility. This form is designed to help organize the required information in a concise way, to streamline the process necessary for approvals and decisions, and thereby facilitate rapid cleanup of a facility.

Major sections of the RAP are:

1. Facility Information. Includes basic information on the facility's location, facility use, and land use in the surrounding area.

2. Contamination. Concisely summarizes the event, initial actions, and meteorological details.

3. Project Team. Includes the member names of the Unified Command and Technical Working Group, as well as any other relevant contacts.

4. Work to Date. Briefly summarizes the characterization sampling and initial air monitoring.

5. Proposed Decontamination Action. The most detailed and extensive portion of the RAP. Many difficult decisions are documented and justified in this section. Examples include: what items will be removed or decontaminated in place, how areas will be sealed off, what decontamination technologies will be used, justifications for using these decontamination technologies, how the effectiveness of decontamination will be monitored, and what monitoring will be conducted to ensure there is no release of decontaminant. It is anticipated that contractors will be called in to provide this information and perform the work.

6. Waste Disposal. Discusses the disposal procedures for waste generated from the decontamination process.

7. Safety. At a minimum refers to the Health and Safety Plan (HASP).

8. Clearance Sampling. At a minimum refers to the Clearance Sampling and Analysis Plan (SAP).

9. Ambient Air Monitoring Plan. At a minimum refers to the Ambient Air Monitoring Plan (AAMP).

10. Schedule. Discusses any schedules planned for the decontamination process. 


\section{APPENDIX J}

\section{Remediation Action Plan}

\section{Facility Information}

\subsection{Facility name, location, address, and owner}

Facility name:

Location (attach figures, if applicable, as Figures 1.1-1 through 1.1-x):

Address:

\subsection{Facility use and physical description}

Facility use:

Auxiliary use or tenants:

Physical description of facility:

Access to facility:

Topography around facility:

Describe any special security issues:

Specialized equipment or items requiring special attention:

Describe the type and availability of facility information that can be used in developing the Remediation Action Plan (e.g., CAD drawings, floor plans, HVAC system descriptions, videos):

\subsection{Land use in the vicinity}

General description of land use within a 2-mile radius: 


\section{Contamination}

\section{$2.1 \quad$ Event}

Time, date, and location of the event:

Describe discovery of release:

Estimated number of people occupying the facility at the time of release:

\subsection{Initial Actions}

Describe HVAC or other airflow changes and time of changes:

List any measures to seal off areas (containment):

Discuss management and evacuation of workers and the public (where moved to, how tracked, any decontamination procedures implemented):

List any preventive measures taken regarding equipment, materials, or items:

Report any initial sampling and results (attach figures and tables, if applicable, as Figures 2.2-1 through 2.2-x and Tables 2.2-1 through 2.2-x, respectively):

\subsection{Meteorological Information}

Wind direction and speed at the time of the event:

Temperature, humidity, cloud cover, precipitation at the time of the event:

Significant changes in meteorological parameters since the event:

Anticipated meteorological parameters at the time of decontamination based on historical trends: 


\section{APPENDIX J}

\section{Project Team}

\subsection{Unified Command Structure}

List name, title, and affiliation of each individual on the Unified Command:

\subsection{Technical Working Group}

List name, title, and affiliation of each individual on the Technical Working Group:

\subsection{Additional Support}

List name, title and affiliation for other key functions or contacts: 


\section{Work to Date}

\subsection{Characterization Sampling}

Dates of characterization sampling:

Overall sampling strategy and rationale:

Sample locations, sample types (methods) and numbers (attach maps, if applicable, as Figures 4.1-1 through 4.1-x):

Sample collection method(s):

Analytical method(s) and laboratory:

\subsection{Characterization Results}

Sample results (attach tables, as applicable, as Tables 4.2-1 through 4.2-x):

Summarize results, and describe areas that need remediation and/or further investigation:

\subsection{Initial Contaminant Air Monitoring (if appropriate)}

Overall sampling strategy and rationale:

Identify locations, sample types (methods), and numbers (attach maps, if applicable, as Figures 4.3-1 through 4.3-x):

Sample collection method(s):

Sample collection frequency:

Analytical method(s) and laboratory:

\subsection{Initial Contaminant Air Monitoring Results (if applicable)}

Sample results (attach tables, if applicable, as Tables 4.4-1 through 4.4-x):

Summarize results, and describe the areas that need remediation and/or further investigation: 


\section{APPENDIX J}

\section{Proposed Decontamination}

\subsection{Offsite Decontamination of Removed Items or Wastes}

List the characteristics and quantities of items to be removed for treatment elsewhere (attach tables, if applicable, as Tables 5.1-1 through 5.1-x; refer to Section 3.5.1 of the Remediation Guidance document):

Describe the method for safely removing these items from the contaminated area:

Describe the proposed off-site technologies (refer to Sections 3.5.2 of the Remediation Guidance document):

Discuss the rationale for using these technologies (refer to Section 3.5.2 of the Remediation Guidance document and Appendix F):

List contact information for the state solid-waste management regulators, disposal facilities (e.g., landfills, incinerators, autoclaves), and/or wastewater treatment facilities that will be accepting the waste:

Crisis Exemption required? (Refer to Appendix F):

\subsection{Onsite Surface Decontamination Technology}

Locations of surfaces to be treated onsite (attach figures, if applicable, as Figures 5.2-1 through 5.2-x):

Describe the proposed onsite surface decontamination technologies (refer to Section 3.5.2 of the Remediation Guidance document and Appendix F):

Discuss the rationale for using these technologies (refer to Section 3.5.2 of the Remediation Guidance document and Appendix F):

Crisis Exemption required? (Refer to Appendix F):

\subsection{Onsite Surface Decontamination Technology}

Locations of areas to be decontaminated (attach figures, if applicable, as Figures 5.3-1 through 5.3-x):

Size of area to be treated onsite (refer to Data Supplement B):

Describe the proposed onsite gaseous technologies (refer to Section 3.5.2 of the Remediation Guidance document and Appendix F): 


\section{APPENDIX J}

Discuss the rationale for using these technologies (refer to Section 3.5.2 of the Remediation Guidance document and Appendix F):

Describe need and status of Crisis Exemption (refer to Appendix F):

\subsection{Pre-Decontamination Work}

List items being removed for disposal (attach tables, if applicable, as Tables 5.4-1 through 5.4-x):

Describe method for safely removing these items from the contaminated area:

Describe methods to seal off or contain contaminated areas (refer to Sections 2.2.7 and 3.4 of the Remediation Guidance document):

Describe methods for additional partitioning of contaminated areas, if necessary (refer to Section 2.2.6 of the Remediation Guidance document):

Describe methods to isolate sensitive electronic equipment (refer to Section 3.5.2.3 of the Remediation Guidance document):

If using liquid reagents, describe measures to collect and dispose of liquid wastes:

Describe measures used to confirm that the areas/equipment are sealed off:

Describe power and other general site requirements:

\subsection{Proposed Gas or Vapor Decontamination Method}

Describe the parameters required to ensure effectiveness (e.g., temperature, relative humidity, fumigant concentration, and contact time):

Describe any safety precautions that need to be employed (include PELs, etc.):

Describe the generation system:

Describe the distribution system (attach figures, if applicable, as Figures 5.5-1 through 5.5-x):

Describe any required pre-tests for parts or all of the system (e.g., low-level performance tests):

Describe any special considerations (such as blocking out light): 


\section{APPENDIX J}

Describe the process for decontamination, and any phasing of the work:

\subsection{Monitoring Effectiveness of Decontamination}

List and describe any sensors, monitors, and chemical and biological indicators used to monitor the effectiveness of the decontaminant:

Indicate locations of sensors, monitors, and chemical and biological indicators used (attach figures, if applicable, as Figures 5.6-1 through 5.6-x):

List analytical laboratories to be used, laboratory requirements, and necessary quality assurance/quality control (QA/QC):

\subsection{Air Monitoring to Ensure No Release of Decontaminant}

List and describe any sensors or monitors used to detect and measure any of the decontaminant leaving the confined decontamination area:

Indicate locations of sensors or monitors used (attach figures, if applicable, as Figures 5.7-1 through 5.7-x):

Describe equipment calibration:

Describe sampling frequency and data collection methods:

Describe analytical methods used, QA/QC, and detection levels if applicable:

Describe the criteria (e.g., air monitoring results) that would trigger a response action. Describe the resulting response action: 


\section{APPENDIX J}

\section{Waste Disposal}

Attach Waste Disposal Plan (refer to Appendix G).

Generate rough estimates for quantities and characteristics of:

- Debris that might need to be removed from hot zones and disposed.

- Waste generated from personnel entering hot zones (e.g., PPE and personnel rinsate).

- Waste produced because of the decontamination technology of choice (e.g., scrubber water).

List contact information for important waste-disposal stakeholders (e.g., state solid waste official, local POTW representative, potential landfills, incinerators, medical waste disposal facilities).

Discuss pre-treatment and disposal options for various types of wastes.

Discuss waste-storage and waste-holding temporary areas, isolation and security for waste-storage areas (describe specific needs, such as refrigeration storage):

\section{Safety}

Attach applicable Health and Safety Plan (refer to Section 2.2.1 of the Remediation Guidance document):

\section{Clearance Sampling and Analysis}

Attach applicable clearance Sampling and Analysis Plan (refer to Section 4.2 of the Remediation Guidance document and Appendixes D and E):

\section{Ambient Air Monitoring Plan}

Attach applicable Ambient Air Monitoring Plan (refer to Section 4.4 of the Remediation Guidance document):

\section{Schedule}

Discuss proposed timeframe for the various stages of the decontamination process:

\section{References (if applicable)}





\section{APPENDIX K}

\section{Annotated Clearance Sampling Plan Template}

The complete clearance environmental sampling strategy is documented in the post-decontamination sampling and analysis plan (Clearance SAP), which must include certain elements. Part 1 of this template outlines the background elements that establish the context for the Clearance SAP. Part 2 suggests an approach to decide upon and document the actual sampling activities. Part 3 provides supporting reference information. The objective of the template is to help ensure that no important information is omitted during planning, and to save time by providing a starting point for developing a written Clearance SAP.

\section{Background Information}

\subsection{Brief Introduction}

Write a brief overview of the situation. Copy, update, and insert any overview information relevant to this incident if already written for other purposes, such as for Appendix I or J.

\subsection{Statement of Authority}

Describe applicable state and Federal laws under which the cleanup activity takes place. Include applicable agreements with, or directives issued by, relevant state or Federal agencies.

\subsection{List and Description of Involved Parties}

Refer to the concept of operations shown in Figure 1-4, and identify the organizations and individuals responsible for developing and performing the Clearance SAP. Include:

- Incident Commander or Unified Commander (IC or UC).

- Sampling group (Operations section).

- Environmental unit (Planning section).

- Technical Working Group (Environmental unit).

- Environmental Clearance Committee (ECC).

- Technical consultants (e.g., analytical chemist, dispersion modeler, statistician, industrial hygienist, toxicologist, structural engineer, HVAC engineer, and fumigation engineer).

\subsection{History of the Event and Actions to Date}

Summarize relevant characterization results, or refer to a written characterization report if one is available. Summarize the decontamination work. Refer to a decontamination report for more details, if one is available. If a decontamination report is not yet ready, describe the decontamination work in detail. 


\section{APPENDIX K}

\subsection{Define the Clearance Process}

For environmental sampling:

- State the clearance criteria (desired environmental conditions after decontamination). Clearance criteria follow from the purpose. For example, if the purpose is to "ensure the airport can be reoccupied," what criteria would be used to make that decision?

- Specify the decision rules (how to decide whether the clearance criteria have been met).

- Deciding that exposure is not acceptable is not the same as deciding that exposure will not occur. Clearance criteria are distinct from decision rules.

- To the extent necessary, there should be a rule for each type of sampling to be used (i.e., a rule for surface sampling large floor areas, one for sampling water fountains, one for bulk samples of soil in decorative planters, and so forth).

- The benefit of a well-designed clearance sampling process is that if decision rules are well defined, interpreting the data and making decisions will be simple and straightforward.

- Specify how to collect and analyze samples. (See Part 2 of this appendix.)

For agents that aerosolize, aggressive air sampling follows environmental surface sampling.

\section{Clearance Sampling}

\subsection{Develop Enviornmental Sampling Plan for Each Sampling Zone}

Use the clearance sampling zone forms in Appendix H to complete the following steps for each sampling zone:

3. Assess available information from the Characterization and Decontamination Phases.

4. For each sampling unit in the sampling zone, decide whether or not to sample that unit.

5. For each sampling unit being sampled, choose a sampling strategy (i.e., targeted, biased, random, or statistical sampling). See Appendixes D and E.

\section{2. $\quad$ Aggressive Air Sampling}

Plan any necessary aggressive air sampling. Describe the placement of air samplers in three dimensions. Describe how the air will be agitated. Specify the duration of the process. Specify the minimum required sampled air volume for each sampler, and the required total sampled air volume (at least two room volumes). 


\section{APPENDIX K}

\section{Supporting Information}

\subsection{Sampling Protocols}

Provide detailed instructions for how to collect samples. Protocols may be written in full, or incorporated by reference. Include all methods that will be used. See Appendix C.

\subsection{Quality Control Samples To Be Collected}

Specify types of quality control samples required (e.g., collocated samples, trip blanks, pure blanks, and spikes). Quality control may be the responsibility of contractors.

\subsection{Sample-Handling Protocols}

Specify how to collect, package, and document each sample, and how to deliver samples to the laboratories. Include how sample handling is incorporated in environmental safety and health ES\&H. See Appendix C.

\subsection{Analytical Methods}

Specify how the laboratories are to analyze the samples. Include quality control procedures required of the laboratories. See Appendix C.

\subsection{Data Management}

Describe sample-naming conventions, which may have been developed as part of the airport's pre-planning. If not, develop them now. Specify how results will be received from laboratories and linked to sample collection information, how results will be stored (e.g., computer database), and facilities for data review. Data management may be the responsibility of contractors.

\subsection{Data Validation}

Summarize how results will be assessed for data quality. Data validation may be the responsibility of contractors.

\subsection{Sampling Design}

Describe rationales of the selected sampling strategies, i.e., the reasoning leading to choices of where to sample and how many samples to collect. Focus on concept. Full technical details, especially of probability-based designs, can be incorporated by reference.

\subsection{Environmental Safety and Health (ES\&H)}

Describe the levels of personal protective equipment (PPE) required for various sampling activities. Describe other actions necessary to ensure safety and health. Refer to the written Health and Safety Plan (HASP) where appropriate. 



\section{APPENDIXI}

\section{Cleanup Contact List}

Airport decision makers should identify the characterization resources shown in Table L-1 in advance of a biological warfare agent (BWA) incident so that such resources can be mobilized immediately. Verify the contact names and phone numbers at least once a year.

Table L-1. Site characterization resources that should be identified in advance.

\begin{tabular}{|c|c|c|}
\hline Resource & Contact & Phone \\
\hline \multicolumn{3}{|c|}{ Members of Unified Command or organization in charge } \\
\hline \multicolumn{3}{|l|}{ Members of Technical Working Group } \\
\hline \multicolumn{3}{|c|}{ Members of Environmental Clearance Committee } \\
\hline \multicolumn{3}{|l|}{ Secondary LRN analytical laboratory } \\
\hline \multicolumn{3}{|l|}{ Sampling team(s) and contractor(s) } \\
\hline \multicolumn{3}{|c|}{ Agent air monitoring team and contractor } \\
\hline \multicolumn{3}{|c|}{ Personal protective equipment (PPE) rental } \\
\hline \multicolumn{3}{|c|}{ Facility engineering and construction team(s) } \\
\hline \multicolumn{3}{|c|}{ Air-transport modeling team and contractor } \\
\hline \multicolumn{2}{|c|}{ Centers for Disease Control and Prevention (CDC) } & \\
\hline
\end{tabular}




\section{APPENDIX}

Airport decision makers should identify the decontamination agencies, teams, and technical contacts shown in Table L-2 in advance of a BWA incident so that such resources can be mobilized immediately. Verify the contact names and phone numbers at least once a year.

Table L-2. Site decontamination resources.

\begin{tabular}{|l|l|l|}
\hline \multicolumn{1}{|c|}{ Resource } & \multicolumn{1}{|c|}{ Contact } & Phone \\
\hline Facility engineering and construction team(s) & & \\
\hline $\begin{array}{l}\text { Decontamination and fumigation team (may } \\
\text { include decontamination reagent suppliers) }\end{array}$ & & \\
\hline National Decontamination Team (USEPA) & & \\
\hline Primary LRN analytical laboratory & & \\
\hline Secondary LRN analytical laboratory & & \\
\hline $\begin{array}{l}\text { Sampling team(s) and contractor(s) for placement, } \\
\text { collection of biological indicators }\end{array}$ & & \\
\hline Centers for Disease Control and Prevention (CDC) & & \\
\hline Personal protective equipment (PPE) rentals & & \\
\hline State solid-waste management division & & \\
\hline Local wastewater treatment facility & & \\
\hline
\end{tabular}


Donald Bansleben

Chemical/Biological Division

Science and Technology Directorate

U.S. Department of Homeland Security

Donald.Bansleben@dhs.gov

\section{Erica Canzler}

Office of Emergency Management

U.S. Environmental Protection Agency

Canzler.Erica@epamail.epa.gov
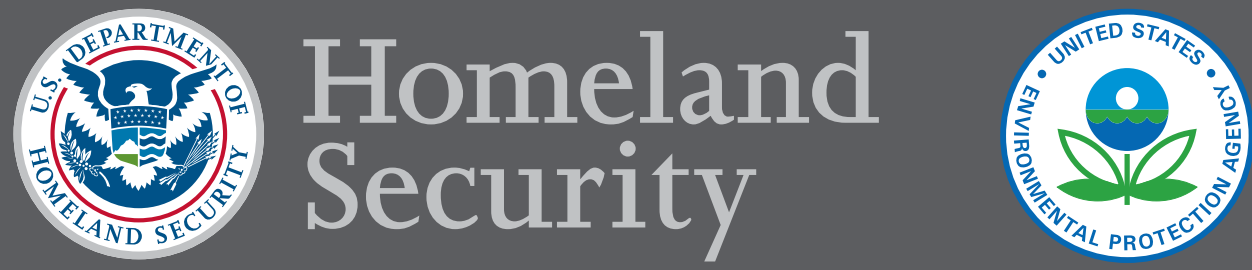